\title{
Current Fluctuations in Hybrid-Superconductor Normal Structures with Quantum Dots
}

\author{
by \\ Stephanie Droste \\ A thesis \\ submitted to the Victoria University of Wellington \\ in fulfilment of the \\ requirements for the degree of \\ Doctor of Philosophy \\ in Physics
}

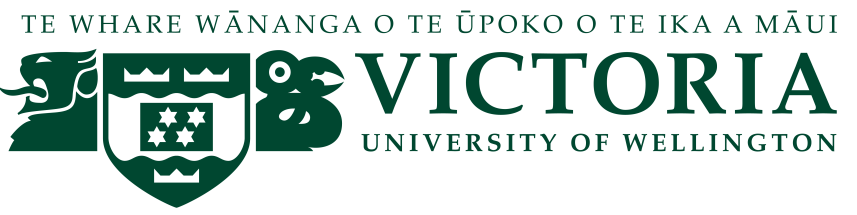

Victoria University of Wellington 2015 



\begin{abstract}
Nanostructures with quantum dots in proximity to superconducting electrodes are an ideal tool to study superconducting correlations in systems with few degrees of freedom that exhibit strong Coulomb-interaction effects. Such hybrid superconductor-normal structures show rich physics due to the interplay of superconductivity, Coulomb interaction and non-equilibrium. Superconducting correlations are established on the quantum dot when it is coupled to a superconductor even in the presence of strong Coulomb repulsion and Cooper pairs can tunnel coherently between the quantum dot and the superconductor.

In this thesis, we investigate theoretically electronic transport through an interacting quantum dot coupled to normal and superconducting leads. The presence of the proximity effect can be detected by the dot's current, namely the Andreev current. However, current fluctuations might reveal information on the electronic transport and the internal structure of the system which is not visible in the mean value of the current. For this reason, we study the current fluctuations through the proximized quantum dot to get access to the properties of such a hybrid quantum-dot system. In particular, we are interested in the finite-frequency fluctuations to unveil the coherent dynamics underlying the proximity effect in the quantum dot and its internal time scales.
\end{abstract}

At first, we present a study of the frequency-dependent current noise for subgap transport through an interacting single-level quantum dot tunnelcoupled to normal and superconducting leads. For this purpose, we employ a non-equilibrium diagrammatic real-time approach to calculate the finitefrequency current noise. The finite-frequency noise spectrum shows a sharp 
dip at a frequency corresponding to the energy splitting of the Andreev bound states which is a signature of the coherent exchange of Cooper pairs between the quantum dot and the superconductor. Furthermore, in the high frequency regime, the so called quantum noise regime, the noise spectrum exhibits steps at frequencies equal to the excitation energies. These steps can be related to the effective coupling strength of the excitations.

However, the statistical description of the electron transport does not stop with the noise. Current cumulants of arbitrary order can be obtained by means of full counting statistics (FCS). We set up a theory based on the diagrammatic real-time approach to calculate the finite-time FCS for quantum transport with a non-Markovian master equation that captures the initial correlations between system and reservoir. This allows us to fully describe the current fluctuations of the hybrid quantum-dot system, that is the noise and all higher order current cumulants. 


\section{Acknowledgments}

As I am now approaching to the end of my $\mathrm{PhD}$, I want to thank everyone who contributed to this work and supported me during the last three years.

First of all, I would like to thank my supervisor Michele Governale for having me as his $\mathrm{PhD}$ student. Throughout his mentoring he was always open for discussions and helped me bringing forward my understanding of physics. I am also very grateful for the many opportunities he has given me to present my work on conferences and workshops.

Secondly, I deeply thank Janine Splettstoesser for a fruitful collaboration and her patient supervision since almost five years. I also like to thank her whole group for hosting and making me feel welcome during a three-month visit at the RWTH Aachen University.

Furthermore, I would like to thank all past and present TCMM group members. Especially, I would like to acknowledge Uli Zülicke for assuming the role as my secondary supervisor.

Many thanks to the MacDiarmid Institute for Advanced Materials and Nanotechnology for funding through a three-year doctoral scholarship.

Moreover, I would like to thank all my friends and office mates for the fine time spent together in Wellington and for making my final period as a student an unforgettable experience. Many thanks to all my friends abroad for their support and encouragement through email and skype. 
Lastly, I like to thank my family for their love and support. Most importantly, a particular thank you belongs to my parents for their encouragement especially in the final period of my $\mathrm{PhD}$ and their constant support during all of my studies.

Stephanie Droste

Wellington

March, 2015 


\section{Contents}

1 Introduction 1

1.1 Hybrid superconductor-normal structures with quantum dots . 1

1.2 Objectives and scope . . . . . . . . . . . . 4 4

1.3 Research methodology ............... 5

1.4 Outline........................ 5

2 Background and basic concepts $\quad \square$

2.1 Quantum dots .................. 7

2.2 Electronic transport through quantum dots . . . . . . . 8

2.3 Coulomb blockade . . . . . . . . . . . . . . 11

2.4 Current fluctuations: noise and full counting statistics . . . . 18

2.4.1 Noise . . . . . . . . . . . . . . . . . . 19

2.4.2 Full counting statistics . . . . . . . . . . . . 22 2

2.5 Superconductivity, Proximity effect, Andreev transport . . . 24

2.5.1 BCS-theory . . . . . . . . . . . . . . 26

2.5.2 Andreev reflection . . . . . . . . . . . . 28 28

2.5.3 Josephson effect . . . . . . . . . . . . . . 2. 29

2.6 Quantum dots coupled to superconductors . . . . . . . . . 32

3 Diagrammatic real-time approach 41

3.1 Model . . . . . . . . . . . . . . . . . . . . . 4 42

3.1.1 Effective Hamiltonian . . . . . . . . . . . . . . . . 44

3.2 Master equation . . . . . . . . . . . . . . . . . . 46 
3.2.1 Density matrix . . . . . . . . . . . . . 46 46

3.2.2 Generalized master equation . . . . . . . . . . . . 47

3.2.3 Master equation in frequency space . . . . . . . . . 53

3.3 Current . . . . . . . . . . . . . . . . . . 54

3.4 Finite-frequency noise . . . . . . . . . . . . . . 557

3.5 Finite-time full counting statistics . . . . . . . . . . . . . 61

3.6 Multi-time full counting statistics . . . . . . . . . . . . . . . 69

4 Finite-frequency noise in transport through a quantum dot 75

4.1 Single-level quantum dot coupled to normal-conducting leads . 76

4.1.1 Non-interacting single-level quantum dot . . . . . . . 77

4.1.2 Noise spectrum for a finite on-site Coulomb interaction 84

4.2 Quantum dot tunnel-coupled to normal and superconducting leads . . . . . . . . . . . . . . . . . . . 88

4.3 Andreev current . . . . . . . . . . . . . . . . . . . 89

4.4 Results for the finite-frequency noise . . . . . . . . . . . 91

4.4.1 Noise in the unidirectional transport regime . . . . . 9 93

4.4.2 Zero and low bias regime, $\mu_{\mathrm{N}}<E_{+,-} \ldots \ldots$. . . . 97

4.4 .3 Finite-bias regime . . . . . . . . . . . . . . . 103

4.5 Conclusions . . . . . . . . . . . . . . . . . . . . . . . . . . . . . 110

5 Finite-time full counting statistics 113

5.1 Unidirectional transport regime . . . . . . . . . . . . . . 114

5.1 Long-time limit . . . . . . . . . . . . . . . . 115

5.1 .2 Finite-time regime . . . . . . . . . . . . . . 116

5.2 Conclusions . . . . . . . . . . . . . . . . . . . . 121

6 Finite-frequency skewness $\quad 123$

6.1 Low-frequency regime . . . . . . . . . . . . . . . . . . . . 124

6.2 Intermediate-frequency regime . . . . . . . . . . . . . . . 128

6.3 Conclusions . . . . . . . . . . . . . . . . . . . . . . 131

7 Summary and outlook 133 


\section{Appendices}

Appendix A Scattering approach for finite-frequency noise 141

A.1 Scattering formalism . . . . . . . . . . . . . . . . . 141

A.2 Results . . . . . . . . . . . . . . . . . . . . . . . . 143

Appendix B Examples for diagrams 145

B.1 Diagrams contributing to the

finite-frequency noise . . . . . . . . . . . . . . 145

B.2 Diagrams contributing to the finite-time FCS . . . . . . . 151

Appendix C Finite-frequency noise in the unidirectional transport regime 


\section{Chapter 1}

\section{Introduction}

\subsection{Hybrid superconductor-normal structures with quantum dots}

In the last decades the miniaturization of electronic devices experienced a substantial progress. Consequently, fundamental building blocks of electronic devices reduced rapidly in size and have nowadays a dimension at the nanometer scale. Due to this size, quantum and charging effects play an important role. The confined electrons of such a small scale device behave quantum mechanically. Hence, the excitation spectrum consists of discrete energy levels. Quantum dots, often called artificial atoms [1] due to their properties, are such quasi-zero dimensional systems. The motion of single electrons is constricted in all three spatial directions due to the size of the quantum dot. Recently, nanostructures with quantum dots in proximity to superconducting electrodes have attracted attention. They are an ideal playground to study superconducting correlations in systems with few degrees of freedom that exhibit strong Coulomb-interaction effects [2, 3]. In superconductors a pair of electrons bind to so-called Cooper pairs due to a weak attraction. The Coulomb repulsion between those Cooper pair electrons is usual rather small due to their spatial separation. Hence, fabricating 
quantum dots in a hybrid superconductor-normal structure, such that it is possible to establish superconducting correlations on the quantum dot even in the presence of a strong Coulomb repulsion, allows the interplay of these two effects.

Intriguingly, these types of structures are at the heart of recent proposals to generate Majorana-fermion excitations in quantum dots [4 7] and to establish and detect different symmetries of superconducting pairing in a controllable way [8]. Another line of research has focussed on the possibility to use double quantum dots, tunnel coupled to superconductors as a source of entangled electron pairs 9,10$]$. It is therefore of vital importance to gain access to the properties of such hybrid quantum-dot systems.

The formation of Cooper pairs and their condensation into a boson-like state in superconductors leads to an energy gap in the density of states of the superconductor. Subgap transport through electronic structures made of superconductors in contact to normal conducting materials is described by means of Andreev reflection [11. At the interface between a normal conducting material and a superconductor the transport is carried by Cooper pairs due to Andreev reflection processes and not due to single electrons. Superconductivity is induced in the normal metal and a energy gap will form in the density of states of the metal, known as Proximity effect.

The properties and internal dynamics of hybrid quantum-dot systems can be accessed by studying the electronic transport. The current gives information on the average number of electrons flowing through a device in a given time. This has been measured in various experimental studies where the subgap spectrum of hybrid superconductor-quantum dot devices has been analyzed by Andreev level spectroscopy 12,22 , which allows to measure the Andreev addition energies and the total line width of the resonances via the differential conductance. Hence, Andreev level spectroscopy detects the presence of the proximity effect.

However, the measurement of the current in experiments is accompanied by fluctuations, which can be quantified by the current noise. These fluctua- 
tions can be of different nature: some arise due to external influences as e.g. $1 / f$ noise or due to vibrations in the measurement setup, while the other part of fluctuations is of an intrinsic nature. The first type of fluctuations has a negative impact on the measurement and generally, the aim is to keep these small. On the other hand the second type of fluctuations, the intrinsic ones, can be a source of information that is not present in the time average value of the current. The intrinsic noise, which arises due to the stochastic nature of the quantum transport in nanoscale conductors, persists even if all fluctuations due to external influences are eliminated.

The intrinsic noise in mesoscopic devices has different origins. The first source arises due to the thermal fluctuations of occupation numbers in the electronic reservoir. The thermal noise in a conductor is an equilibrium phenomenon and gives information on the temperature and the conductance [23]. A second source of intrinsic noise appears due to the randomness in transport across the nanostructure. To get more useful details out of the noise spectrum one has to apply a nonzero voltage, which lets the noise rise above its equilibrium value and makes the noise frequency dependent. The so called shot noise occurs due to the discreteness of the electrical charge. Both thermal and shot noise are classical concepts. Additionally, in the high-frequency regime, so called quantum noise emerges from zero-point fluctuations [24].

However, to obtain further understanding on the electron transport it might be useful to study not only the current noise but also higher order cumulants. The extraction of the higher moments directly from the current fluctuations in experiments is a demanding task and has been achieved only for the third order cumulant 25]. Alternatively higher moments to arbitrary order are directly accessible in terms of the Full Counting Statistics (FCS) [26, 27]. FCS is nowadays an established concept not only in theoretical but also in experimental physics since it became feasible to measure the FCS in transport through a quantum dot 28]. Current fluctuations and FCS are usually studied in terms of a long-time measurement. The long-time limit is equivalent to zero-frequency noise or rather shot-noise for a system 
out of equilibrium.

In order to extract additional information concerning the internal dynamics and their characteristic time scales, it is valuable to study the current fluctuations for finite times. That is the finite-frequency current noise and even higher orders of the current correlation function, as well as the finite-time FCS. Indeed, the non-equilibrium finite-frequency noise of quantum dots in different regimes and setups has previously been at the focus of various theoretical studies 29 48].

\subsection{Objectives and scope}

The purpose of this thesis is to study the current fluctuations in hybrid superconducting-normal structures with quantum dots in the presence of Coulomb repulsion and superconductivity. The regime in which the tunnelcoupling rate between the superconductor and the quantum dot is strong (larger than the coupling to the other normal-conducting leads eventually present in the device) is considered. In this regime it is possible to establish a BCS-like state in a single-level quantum dot even in the presence of strong Coulomb repulsion [49]. Such a state is characterised by a coherent exchange of Cooper pairs between the dot and the superconducting lead. In the two-terminal case of a quantum dot, tunnel-coupled to one normal and one superconducting lead, the proximity effect is established by generating a non-equilibrium situation by means of an applied transport voltage. Hence, this system shows rich physics due to the combined effect of Coulomb interaction, superconductivity and non-equilibrium. In order to reveal the coherent dynamics underlying the proximity effect, a quantity of high interest to look at is the finite-frequency noise of the Andreev current. Current noise spectroscopy in mesoscopic systems has become a standard tool to gain information on the transport processes and internal time scales of mesoscopic conductors 50 59]. However, there might be even more information on the transport processes which are not contained in the current noise. A full 
statistical description on the electronic transport can be determined by the FCS. In particular we want to reveal the internal dynamics and time scales of the system. Therefore, finite-time FCS $[40,60-63]$ is a suitable quantity to fully access the short time behavior of the current fluctuations.

\subsection{Research methodology}

In order to study electronic transport and in particular the current fluctuations through a quantum dot we make use of a diagrammatic real-time approach. The diagrammatic-real time transport theory [64 66] is a general transport theory used to describe non-equilibrium transport through a strongly interacting quantum dot weakly coupled to electronic reservoirs. An expansion in the tunnel coupling to the leads is used, which allows to take into account the Coulomb interaction on the quantum dot exactly. The system is described in terms of a reduced density matrix. Based on the diagrammatic real-time approach we determine the current and the finite-frequency noise through a quantum dot strongly coupled to a superconductor and weakly coupled to a normal-conducting lead. The FCS gives a full statistical description of the electron transfer through the quantum dot. To reveal further information on the coherent dynamics of the system we set up a theory to obtain the finite-time FCS.

\subsection{Outline}

This thesis is organized as follows: In Chapter 2 we present the basic concepts and motivation underlying this thesis. First, we introduce quantum dots and the basic concepts of electronic transport through quantum dots. In Sec. 2.4 we give an overview of different types of noise, followed by an introduction of full counting statistics. Next, in Sec. 2.5 we review the basic principles of superconductivity like the BCS theory, Andreev reflection and the Josephson effect. This is followed by a review on experimental achievements in hybrid 
Introduction

superconductor-quantum dot devices.

Chapter 3 is dedicated to the theories we are using to obtain the results of this thesis. This chapter can be safely skipped for everyone who is just interested in the discussion of the results. First we introduce a diagrammatic real-time theory for transport which will be the basis for the subsequent sections. In the next section we introduce a method to obtain the current and the finite-frequency noise. This is followed by a theory to determine the finite-time FCS based on a non-Markovian master equation. This approach allows not only to calculate the current and finite-frequency noise, but gives also a direct access to all higher order current correlation functions. In the last section of Chapter 3 we extend the formalism of finite-time FCS and present a theory of multi-time FCS for Markovian processes, which allows to determine the power spectral density at different frequencies.

In Chapter 4 we present results for the current and frequency-dependent current noise through an interacting single-level quantum dot tunnel-coupled to normal and superconducting leads. We only consider a weak coupling to the normal-conducting lead. For the superconductor we are interested in the subgap features in transport and restrict ourself to the limit of a large superconducting gap $(\Delta \rightarrow \infty)$. Throughout this thesis we will work in the regime of a strong coupling to the superconducting lead, while the normalconducting lead is only weakly coupled. Depending on the relation for applied bias voltage and noise frequency we can access different noise regimes.

Next, in Chapter 5 we apply the theory of finite-time FCS to a single-level quantum dot tunnel-coupled to one normal and one superconducting lead. We present results of the finite-time FCS for this system.

Results for the frequency-dependent skewness for unidirectional transport based on the theory for multi-time FCS introduced in Sec. 3.6 are presented in Chapter 6.

Finally, the main results of this thesis are summarized in Chapter 7 and an outlook of future work is given. 


\section{Chapter 2}

\section{Background and basic concepts}

\subsection{Quantum dots}

A typical quantum dot, made out of semiconducting materials, consists of about $10^{2}-10^{9}$ atoms with length scales ranging in the nanometer to micrometer scale 67 .

Experimentally, quantum dots are often realized by semiconductor heterostructures, where electrons are confined into a submicrometer regime. There exist two types of semiconductor quantum dots, lateral [68,69] and vertical quantum dots [70]. Figure 2.1. (a) shows an example for a lateral quantum dot device and (b) for a vertical quantum dot realization. Quantum dots are quite general mesoscopic systems and there are a lot further realizations beside semiconductor materials. There have been quantum dots investigated based on graphene, see for example Ref. [16, 71,73] or carbon nanotubes, where their natural confinement can be gated to a quantum dot 74 76 or even molecules [77] and single atoms [78,79]. Figure 2.1 (c) shows a typical realization of quantum dots in a nanowire [80,81]. The gate electrodes define the quantum dot in the nanowire. Fig. 2.1 (d) displays an example of a double quantum dot device using a carbon nanotube. Top gates are attached to the carbon nanotube to create the quantum dot by dragging down its valance band. This will form a pool of electrons, i.e. a quantum dot in the vicinity 
Background and basic concepts

of the gates.

The ability to control the properties of quantum dots as their size or the spacing of the levels make them attractive for a various range of applications as nano-electronics [83], quantum computation [84 86] and optics 87]. Numerous properties of quantum dots can be studied by performing an electronic transport measurement. To study transport through quantum dots, they have to be connected to source and drain electrodes as well as to a gate, so that single electrons can tunnel from the electronic reservoir on and off the quantum dot. A current flows across the junction if a voltage difference is applied between source and drain electrode. The gate electrode has no direct electric contact to the quantum dot or one of the transport electrodes. Varying the gate voltage, controls the number of charge states on the quantum dot and therefore its size. A schematic figure of an electronic transport measurement with a quantum dot is depicted in Fig. 2.2. Such a setup is also known as a single-electron transistor. Instead of a quantum dot also a metallic island can be part of the set-up.

The Coulomb repulsion is the dominant energy scale due to the size of the device. Therefore it is the ideal device to study interaction effects and their influence on various transport properties.

In the next section, the basic concepts of quantum transport through a quantum dot are summarized including the theoretical model to describe a single-level quantum dot.

\subsection{Electronic transport through quantum dots}

Under the assumption that the splitting of the energy levels in the quantum dot is large, i.e. much larger than the other energy scales in the system such as the temperature or the applied bias voltage, only one single energy level can be accessed in transport. A simple approach to describe the quantum dot with only one single orbital level was formulated by P.W. Anderson [88]. The Anderson model, which was originally introduced for impurities in metals, 


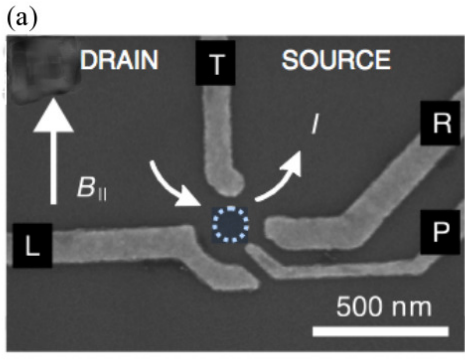

(c)

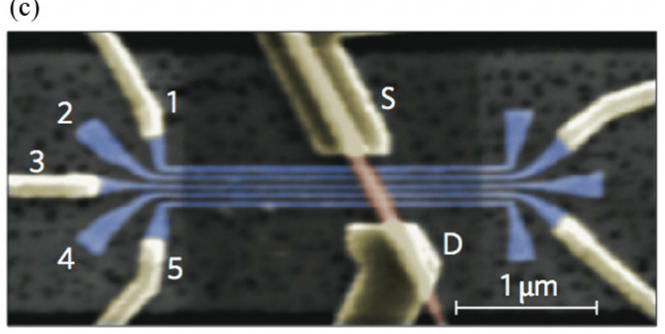

(b)
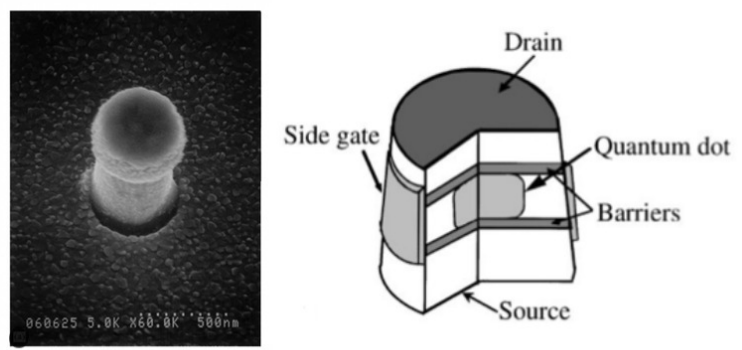

(d)

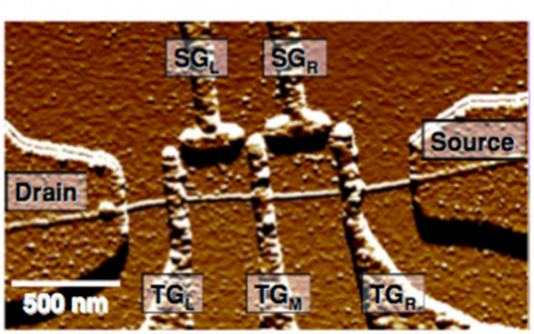

Figure 2.1: (a) Scanning electron micrograph of a lateral quantum dot device. The lateral quantum dot is defined by $\mathrm{Ti} / \mathrm{Au}$ gate electrodes indicated by the white dashed circle, which are lying on top of a GaAs/AlGaAs heterostructure. The picture is taken from Ref. [82]. (b) Scanning electron microscope photograph of a vertical quantum dot (left) and schematic diagram of the device (right). The quantum dot is formed in an In/Ga/As well layer sandwiched by barriers made of $\mathrm{Al} / \mathrm{Ga} / \mathrm{As}$. Reprinted from Physica B, 246-247, 83, Copyright 1998, with permission from Elsevier. (c) Scanning electron microscopy image of an nanowire (InSb) contacted to gates to form quantum dots. The source and drain contacts are made of Ti/Al. Reprinted by permission from Macmillan Publishers Ltd: Nature Nanotechnology 8, 170, copyright 2013. (d) Picture of a carbon nanotube double quantum dot device. The source and drain contacts are made of $\mathrm{Pb}$ and the gate electrodes of Al. Topgates create tunable tunnel barriers to define the quantum dots, while side gates are used to change the chemical potential of the separate dots individually. Reprinted by permission of IOP Publishing: Semicond. Sci. Technol. 21, S52, copyright (2006). 


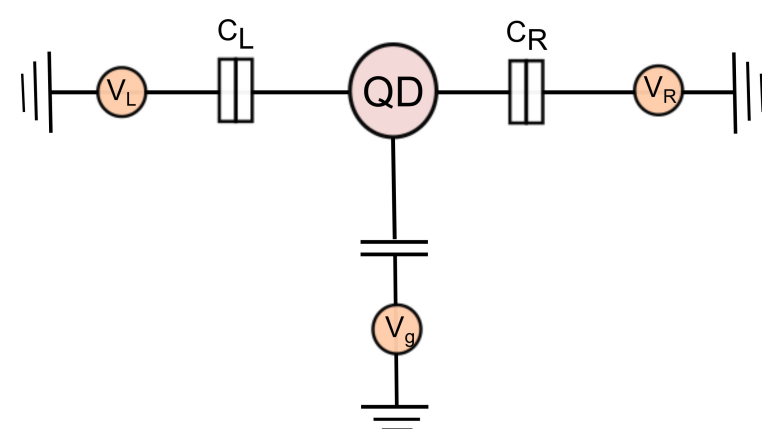

Figure 2.2: Schematic figure of a quantum dot single electron transistor. Electron transport from left to right may be influenced by the gate voltage $V_{\mathrm{g}}$. The boxes represent tunnel barriers with capacitances $C_{\mathrm{L}}$ and $C_{\mathrm{R}}$.

describes the single-level quantum dot by the Hamiltonian

$$
H_{\mathrm{dot}}=\sum_{k \sigma i} \epsilon_{\sigma} n_{\sigma}+U n_{\uparrow} n_{\downarrow}
$$

where $n_{\sigma}$ is the particle number operator $n_{\sigma}=d_{\sigma}^{\dagger} d_{\sigma}$ and $d_{\sigma}^{(\dagger)}$ is the annihilation (creation) operator of an electron with spin $\sigma=\uparrow, \downarrow$ in the quantum dot. The energy level is described by $\epsilon_{\sigma}$, where the index $\sigma$ accounts for spin dependence. The Coulomb interaction on the quantum dot is taken into account by the charging energy $U$. The quantum dot has to be coupled to two electrodes left and right (or rather source and drain electrodes) with chemical potential $V_{\mathrm{L}}$ and $V_{\mathrm{R}}$ to drive a current across the device. The electronic reservoirs are modeled by the lead Hamiltonian,

$$
H_{\mathrm{L}}=\sum_{k \sigma i} \epsilon_{k} c_{k \sigma i}^{\dagger} c_{k \sigma i}
$$

with the operators $c_{k \sigma i}^{\dagger}$ and $c_{k \sigma i}$ describing the creation and annihilation of electrons in the leads $i=\mathrm{L}, \mathrm{R}$ with momentum $k$ and spin $\sigma$. The tunneling of electrons from the electronic reservoirs on and off the quantum dot is possible due to tunnel junctions between the dot and the leads. The tunnel junctions are described by the tunneling Hamiltonian,

$$
H_{\mathrm{T}}=\sum_{\sigma} t_{i k \sigma} c_{k \sigma i}^{\dagger} d_{\sigma}+\text { h.c. }
$$


with the tunnel matrix element $t_{i k \sigma}$. The spin is conserved during the tunneling process, since the Hamiltonian $H_{\mathrm{T}}$ (Eq. (2.3p) is diagonal in $\sigma$. The full Hamiltonian of a quantum dot coupled to normal conducting leads reads then $H=H_{\mathrm{dot}}+H_{\mathrm{L}}+H_{\mathrm{T}}$. We assume that the electronic reservoirs consist of a Fermi sea of electrons with the Fermi function $f(E)=1 /\left(1+e^{\left(E-\mu_{i}\right) / k_{\mathrm{B}} T}\right)$, where $T$ is the temperature with $k_{\mathrm{B}}$ the Boltzmann constant and $\mu_{i}$ the chemical potential of the lead.

The central object to describe the transport properties of quantum devices comprising quantum dots is the current operator, which characterizes the transport properties of such a nanoelectronic device. We are interested in the non-equilibrium transport properties. A measurable current runs through the device after it is brought out of equilibrium via the applied bias voltage, $V_{\mathrm{L}}-V_{\mathrm{R}}$. The current operator across the junction can be defined by the rate change of the number of electrons in the electronic reservoirs,

$$
\hat{I}_{i}=-e \dot{N}_{i}=\frac{i e}{\hbar}\left[N_{i}, H_{\mathrm{T}}\right]
$$

where $N_{i}=\sum_{k, \sigma} c_{i k \sigma}^{\dagger} c_{i k \sigma}$ is the particle number operator for lead $i=\mathrm{L}, \mathrm{R}$. The single-electron transistor, as shown in Fig. 2.2, allows the transfer of electrons one-by-one from the left to the right reservoir. However, the Coulomb interaction opposes the addition of electrons. A current flows only when the incoming electrons have enough energy to overcome the Coulomb repulsion.

\subsection{Coulomb blockade}

The capacitance of a system decreases with its size. Hence, the capacitance of a quantum dot is small and consequently its charging energy is large. Considering a small system like a quantum dot or a metallic island the electronelectron interaction has a significant impact and has to be included into the theoretical description of quantum transport. In the following we provide a concept which describes the influence of the Coulomb repulsion on the electronic transport properties of the quantum dot. The physics of Coulomb 
blockade, which is a classical concept, is an effect of the charge quantization. The functionality of devices like single-electron transistors (SET) is based on single charge transport and its behavior is regulated by the electrostatic energy. A quantum dot or a metallic island can be part of the SET, Fig. 2.2. While the metallic island hosts a continuum of single-particle energy levels (the charging energy is much larger than the level spacing), the quantum dot has a discrete excitation spectrum. Quantum effects may appear due to the discreteness of the energy levels in the quantum dot, which might have additional effects on the transport properties. Nevertheless, both types of SET exhibit the same basic transport properties. We are introducing the classical concept of single-charge tunneling in the following.

The single electron transistor as displayed in the schematic Fig. 2.4 (a) is coupled to two electronic reservoirs and capacitively coupled to a gate. The total capacitance is given by the sum of the different contact capacitances, $C=C_{\mathrm{L}}+C_{\mathrm{R}}+C_{\mathrm{g}}$, with $C_{\mathrm{L}}, C_{\mathrm{R}}$ capacitances arising from the tunnel couplings to the reservoirs and $C_{\mathrm{g}}$ is the gate capacitance. To have transport at least one energy level has to be in the bias window. By adding an electron on the dot, we are changing its energy. All excess electrons tunneling onto the quantum dot have to overcome the energy difference between the charging states. Assume the dot is occupied by $N$ electrons, the charging energy necessary to change the charging state is given by

$$
E_{\mathrm{ch}}\left(N, N_{0}\right)=E_{\mathrm{C}}\left(N-N_{0}\right)^{2}
$$

with $E_{\mathrm{C}}=e^{2} / 2 C$ the charging energy of a single electron. The smaller the system we consider, the smaller the capacitance and consequently, the larger is the energy to add an extra electron on the system. The applied bias voltage induces an external charge $e N_{0}$ on the dot, which is given by

$$
e N_{0}=C_{\mathrm{L}} V_{\mathrm{L}}+C_{\mathrm{R}} V_{\mathrm{R}}+C_{\mathrm{g}} V_{\mathrm{g}}
$$

The voltages $V_{\mathrm{L}}$ and $V_{\mathrm{R}}$ define the chemical potential in source and drain electrodes and $V_{\mathrm{g}}$ is the gate voltage. Figure 2.3 shows the charging energy 
$E_{\mathrm{ch}}$ of the quantum dot as a function of the external charge $N_{0}$ for different occupations of the quantum dot.

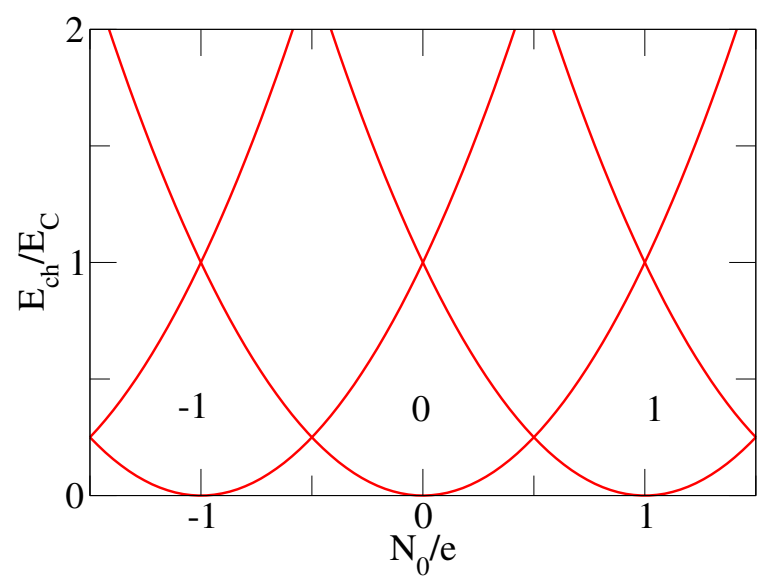

Figure 2.3: Charging energy $E_{\mathrm{ch}}$ as a function of the external charge $e N_{0}$ for different charging states $N$.

The lowest energies in Fig. 2.3 correspond to the ground state. The energy, which is necessary to change the number of electrons occupying the dot by one is maximal if the external charge $e N_{0}$ is a multiple of the elementary charge. In these regions the electronic transport through the quantum dot is blocked. This phenomenon is known as Coulomb blockade. For half-integer values of $N_{0}$, namely at the degeneracy points of the parabolas, no energy is required to change the dot occupation and electron transport is possible independent of temperature or bias voltage. In order to have a current flow across the device, the latter situation is the favorable one to allow electron tunneling on and off the quantum dot.

This behavior from a blocked to a conducting charge state is manifested in the conductance. The differential conductance $(G=\partial I / \partial V(V=0))$ as a function of the gate voltage shows a sharp peak structure due to the Coulomb blockade, as displayed in Fig. 2.4 (b). These so-called Coulomb oscillations take place at the degeneracy points of the charging energy $E_{\mathrm{ch}}$. Hence, electrons can freely jump on and off the quantum dot at the peaks, 
(a)

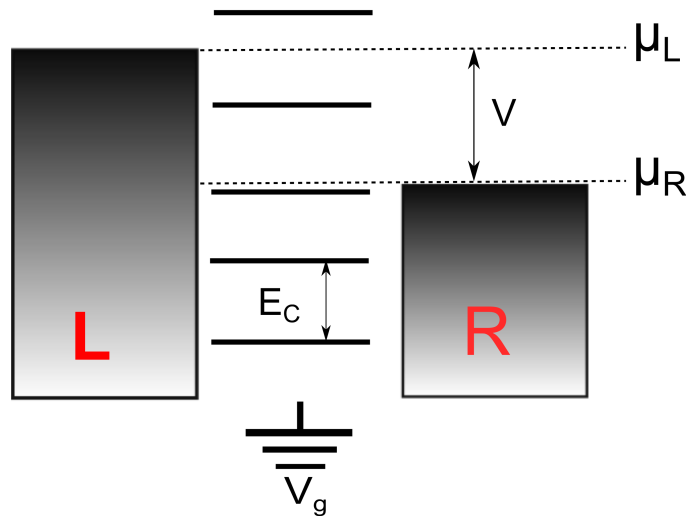

(b)

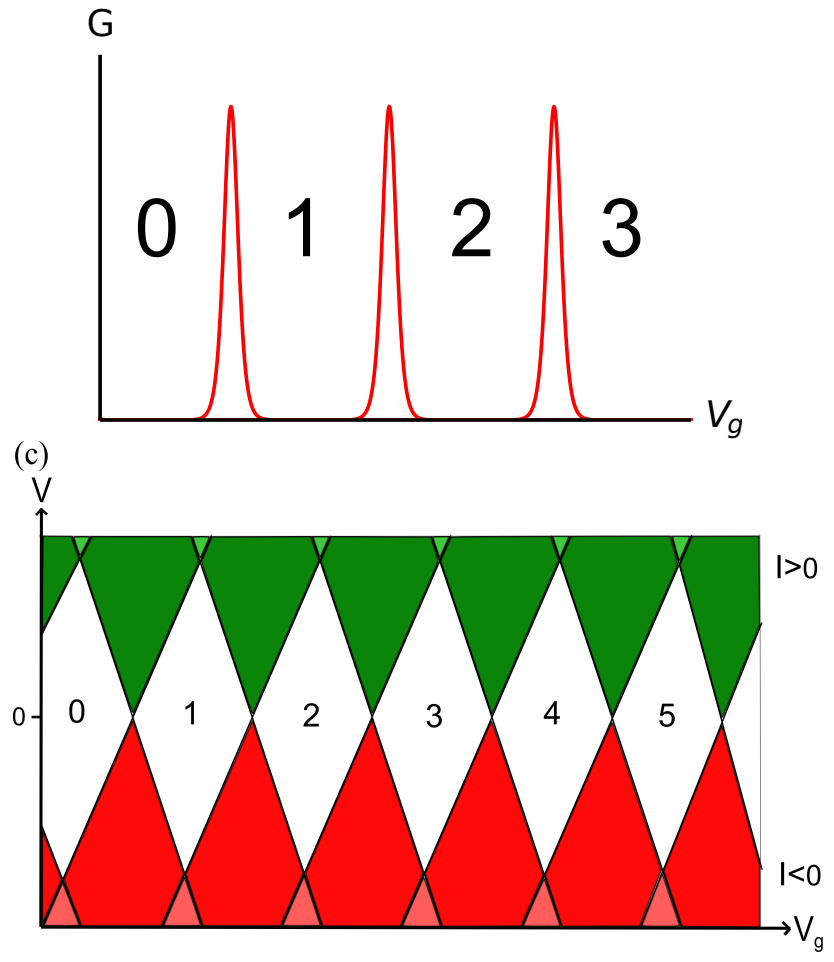

Figure 2.4: (a) Sketch of the energy landscape of the quantum dot. The dot is capacitively coupled to a gate $V_{\mathrm{g}}$, which controls the number of electrons on the dot. The horizontal lines represent the energy levels. The electronic reservoirs, the Fermi distributions, are shown by the bars. (b) Differential conductance $G$ as a function of the gate voltage $V_{\mathrm{g}}$. (c) Schematic plot of the current as a function of bias voltage $V$ and gate voltage $V_{\mathrm{g}}$ through a quantum dot. In the white regions, the so-called Coulomb diamonds, the current is suppressed. The current is positive $(I>0)$ in the green region and negative in the red $(I<0)$. 
while the transport is blocked outside the peaks due to the Coulomb blockade. The width of the peaks is determined by the temperature $k_{\mathrm{B}} T$. However, to ensure that thermal fluctuations do not smear out the charging effects, $E_{\mathrm{ch}} \gg k_{B} T$ has to be fulfilled.

The gate voltage controls the number of electrons on the dot. By varying the gate voltage we shift the energy level structure up or downwards and control the external charge, see Fig. 2.4 (a). On the other hand the applied bias voltage $\left(V=V_{\mathrm{L}}-V_{\mathrm{R}}\right)$ determines the Fermi levels of left and right reservoir and adjust the transport window, i.e. controls the number of dot levels which are accessible in transport. Consequently, bias voltage and gate voltage control whether a finite current flows or if transport is suppressed due to the Coulomb repulsion. Depending on bias and gate voltage a diamond structure occurs in the conductance, the so called Coulomb diamonds. Fig. 2.4 (c) displays the schematic sketch of a current measurement through the quantum dot device as a function of the gate voltage $V_{g}$ and the bias voltage $V$, showing the Coulomb diamonds of the Coulomb blockade [89]. In the white regions the current is suppressed as a result of the Coulomb blockade. Each diamond corresponds to a certain fixed number of electrons. Above a threshold bias voltage, single electron transfer is available. The upper and lower corner of the diamonds are slightly shifted, which indicates that the capacitances between left and right reservoir are asymmetric $C_{\mathrm{L}} \neq C_{\mathrm{R}}$.

As mentioned at the beginning of this section, the concept we have described here is a classical one and also holds for a metallic island instead of a quantum dot being part of the SET. However, in case of a quantum dot there are a great number of extra lines in the $V-V_{\mathrm{g}}$ plane, which occur due to the discreteness of the excitation spectrum. The extra lines manifest discrete states on the quantum dot, ground and excited states. By measuring and analyzing their position one can identify them and hence perform a transport spectroscopy. Figure 2.5 shows an example for an experimental measurement of the differential conductance as a function of bias voltage and gate voltage. The measurement has been performed for a suspended carbon 
nanotube quantum dot. The device is shown in Fig. 2.5 (a). The carbon nanotube is attached to source and drain electrodes, while a top gate defines the quantum dot. The differential conductance Fig. 2.5 (b) shows a series of Coulomb diamonds as well as a great number of extra lines outside the diamonds which can be identified as the excited states. In general, the lines parallel to the diamond edges can be assigned to resonant tunneling and are the excited states. The energy difference gives information on the energy spacing of the states. The tunnel spectroscopy might reveal additional lines, which do not show up in the differential conductance displayed in Fig. 2.5(b). For instance, all lines parallel to the bias voltage $V$ indicate a level crossing inside the dot. In contrast, lines found parallel to the gate voltage axis $V_{\mathrm{g}}$ are an indication of co-tunneling processes, i.e. second order tunneling processes and all lines at zero bias indicate the Kondo regime.

In the transport process of a quantum dot coupled to external electrodes via tunnel junctions, different tunnel processes can arise. In this thesis we consider only a weak coupling of the quantum dot to the electronic reservoirs. In this regime sequential tunneling [90] is the dominant process, if the dot level is within the bias window. In this limit electrons can only enter the quantum dot one after the other.

In the case that no energy level is within the transport window, sequential tunneling is exponentially suppressed and the system is in the Coulomb blockade regime. Tunneling can only occur via a higher order tunnel process as elastic/inelastic co-tunneling processes, where the dot state is virtually occupied. Co-tunneling processes briefly violate the energy conservation, which is allowed by the Heisenberg uncertainty principle.

For a strong coupling, beyond the sequential tunneling regime, the Kondo effect 91 may arise if the temperature is smaller than some critical temperature, the so-called Kondo temperature $T<T_{K}$. This happens favorably if the system is in the singlet state, meaning the dot is occupied with an odd number of electrons 92,93 and bias voltage is close to zero. In the case of a single-level quantum dot, the dot is only occupied with a single electron 
(a)
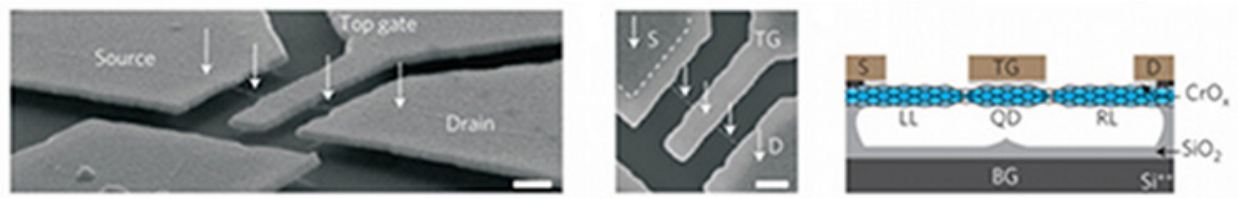

(b)

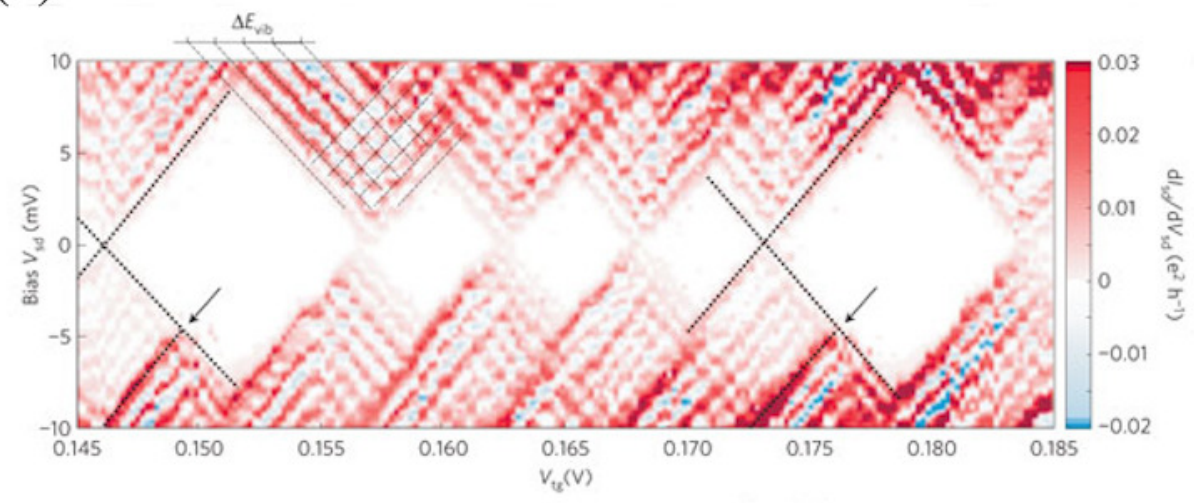

Figure 2.5: (a) Electron microscope micrograph view of a suspended carbon nanotube with source and drain electrodes. The quantum dot is formed in the vicinity of the top gate as shown in the schematic figure on the right. (b) Differential conductance shown as a function of bias voltage and gate voltage. Reprinted by permission from Macmillan Publishers Ltd: Nature Physics 5, 327, copyright 2009. 
and is namely in state $|\sigma\rangle$. The quantum dot spin forms a singlet state with electrons at the Fermi energy of the leads. This leads to a screening of the quantum dot spin by the reservoir spins, which results in a peak in the zerobias conductance [68]. Nevertheless, the Kondo effect is not in the scope of this thesis. Throughout this thesis we will only consider transport regimes where the Kondo effect plays no role 94$]$.

\subsection{Current fluctuations: noise and full count- ing statistics}

As we have discussed in the previous section, an electrical current flows through a quantum dot contacted to electronic reservoirs when a constant bias voltage is applied across the device. The stationary current tells us the average number of electrons which have passed the device per time. The current shows a time dependence and undergoes fluctuations around its average value. Here, we are interested in these current fluctuations, since they can be a source of information that is not present in the time-averaged value. These fluctuations are for instance characterized statistically by the variance of the electron number that has been transported in a given time.

The current fluctuations in a mesoscopic device can have different origins. Some fluctuations arise due to external influences, which have generally a negative influence on the measurement. It should be the aim to keep these small. Another source of fluctuations is of intrinsic nature, which arise due to the stochastic nature of the quantum transport in nanoscale conductors. The intrinsic noise, which persists even if all fluctuations due to external influences are eliminated, might contain information on the transport processes which cannot be extracted from the average current.

One quantity of high interest is the current noise, that is the variance of the number of transported electrons through the device. The current noise in mesoscopic devices has been widely studied in the last two decades, theoretically and experimentally $[32]$. However, the statistical description of the 
electron transport does not stop with the noise. Also higher statistical moment such as the skewness or the kurtosis might contain further information on the transport properties. The full counting statistics (FCS) is able to extract the statistical fluctuations of the current and hence, allows to account for statistical moments of arbitrary order.

Next, we will introduce different contributions to the intrinsic noise and its basic concepts. This is followed by an introduction to FCS.

\subsubsection{Noise}

In general the noise in electrical conductors has many sources, namely external and intrinsic ones. Here, we want to discuss only the intrinsic contributions to the noise, that is the thermal or Johnson-Nyquist noise and the shot or non-equilibrium noise as well as quantum noise. We will show that noise is not only the spurious part of a current measurement, but that the noise can contain further information about the system and the statistics of electron transport. First we will review the basic properties of thermal and shot noise, namely the low-frequency contribution. This is followed by an introduction to noise in the quantum regime (the high-frequency noise).

\section{Thermal and shot noise}

As previously mentioned the variance of the current is related to the noise. The noise is characterized by its spectral density $S(\omega)$, which is the Fourier transform of the autocorrelation function. The finite-frequency noise thus takes the form of the following spectral density,

$$
S(\omega)=\int_{-\infty}^{\infty} d t e^{i \omega t}\langle\delta I(t) \delta I(0)\rangle
$$

where $\delta I(t)=I(t)-\langle I\rangle$ describes the time-dependent fluctuations in the current at a given bias voltage $V$ and temperature $k_{\mathrm{B}} T$. The noise power introduced in Eq. (2.7) is asymmetric with respect to the frequency. However, the noise is often defined as the symmetrized version where $S(\omega)=S(-\omega)$. 
In his pioneering experiment in 1918 Walter Schottky 95] reported that in a vacuum tube under ideal conditions, i.e. all sources of perturbing noise has been eliminated, still two types of noise remained in the electrical current, described by him as thermal and shot noise. The first contribution stems from the thermal excitations of the electrons at finite temperature known as Johnson-Nyquist noise [23] or simply thermal noise. This type of noise occurs in any conductor as long as there is a finite temperature. The JohnsonNyquist noise [23] of a conducting system at a certain temperature $k_{\mathrm{B}} T$ in equilibrium is given by

$$
S(\omega=0)=2 k_{\mathrm{B}} T G,
$$

where $G$ denotes the linear conductance of the system. Hence, the equilibrium zero-frequency current noise can be directly related to the linear response of the current.

The second type of intrinsic noise is the so-called shot noise which arises due to the discreteness of the electron charge, i.e. the electrons arrive at the reservoir in a quantized fashion. While the thermal noise is dominant at equilibrium, shot noise is a non-equilibrium phenomenon and becomes the dominant contribution to the noise, if the energy scale related to the nonequilibrium is much larger than the temperature $k_{\mathrm{B}} T$. For instance in case of electron transport due to an applied bias voltage $V$, the shot-noise becomes dominant for $e V \gg k_{\mathrm{B}} T$.

The corresponding spectral density at zero frequency for uncorrelated events reads,

$$
S(\omega=0)=q\langle I\rangle
$$

with $q$ the charge of the carriers associated to the transport process. The shot noise Eq. 2.9) is proportional to the average current $\langle I\rangle$ flowing through the system and describes a Poissonian process. The ratio $F=S / q\langle I\rangle$ called the Fano factor, named after Ugo Fano's theory of the statistics of ionization [96], allows to measure the unit of transferred charge. For instance, in case of a superconductor-normal metal junction the charge is added to the superconductor in Cooper pairs so, correlated one expect a Fano factor of 
$F=2$, if we have a Poissonian transfer of Cooper pairs. The doubling of the noise has been measured experimentally 97 .

In case of electrons the Fano factor is $F=1$. The Fano factor quantifies also the deviations from the Poissonian value. The deviation from the Poissonian value $F=1$ results in sub-Poissonian $F<1$ or super-Poissonian $F>1$ values of the Fano factor. These deviations occur due to statistical anti-bunching or bunching effects which lead to negative or positive crosscorrelations. In a fermionic system anti-bunching effects, (i.e. the electrons avoid each other and arrive separately at the detector) usually due to the Pauli principle, correlate the electrons and yield sub-Poissonian shot noise. In contrast, in bosonic systems bunching leads to super-Poissonian values of the Fano factor.

We want to point out that the concept of noise we have introduced here is a statistical effect and a classical phenomenon. It can be present in any type of conductor. However, special interest lies in the study of shot noise in mesoscopic conductors such as a quantum dot coupled to electronic reservoirs. Prominent examples with simple universal values showing the sub-Poissonian behavior of the shot noise are a symmetric double barrier $(F=1 / 2)$, a diffusive wire $(F=1 / 3)$ or a chaotic cavity $(F=1 / 4)[32$. While thermal and shot-noise are zero or rather low-frequency noise phenomena, it might be of interest to also study the noise at high frequencies.

\section{Noise in the quantum regime}

The electrical current noise has been extensively studied at low- and zerofrequency. However, the high-frequency noise is still quite unexplored.

The so called quantum noise is dominant for high frequencies, namely when the noise frequency is much larger than temperature and applied bias voltage, $\omega \gg k_{\mathrm{B}} T, V$. Quantum noise arises from zero-point fluctuations in a device. In this regime we can understand the current fluctuations as a exchange of photons of certain energy $\omega$ emitted or absorbed between the source and the detector. Hence, it will allow visualizing of the transport 
processes which are enabled or blocked by energy absorption or emission.

For instance at zero temperature $k_{B} T$ and zero bias voltage $V$, the system will not be able to emit energy, but is able to absorb energy. At finite temperatures the emission of energy is possible up to frequencies $\omega=k_{\mathrm{B}} T$, before the absorption noise sets in. The noise at negative frequencies is referred as the emission noise while the noise at positive frequencies is the absorption noise. Therefore the noise spectrum is asymmetric with respect to the noise frequency $\omega$. If the system is driven out of equilibrium $V>0$, shot noise is also present in the noise spectrum.

In summary, at low and intermediate frequencies, the noise is dominated by thermal fluctuations and shot noise. The thermal noise, which stems from thermal excitations of the electrons, is dominant in the spectrum if $k_{\mathrm{B}} T \gg \omega, V$. In contrast shot noise dominates in the spectrum if the bias voltage is the largest energy scale, $V \gg k_{\mathrm{B}} T, \omega$. Quantum noise is dominant if $\omega \gg k_{\mathrm{B}} T$. In this limit the noise can be interpreted in terms of energy emission or absorption by the noise source [24], which leads to a strong asymmetry in the noise spectrum.

Current noise spectroscopy in mesoscopic systems has become a standard tool to gain information on the transport processes and internal time scales of mesoscopic conductors [50 59. In particular, in order to measure quantum noise it is necessary to measure independently the emission and absorption noise. Quantum noise has been detected experimentally $52,56,57,98,99$.

\subsubsection{Full counting statistics}

In the previous subsection we have introduced the noise, which is a measure of the current fluctuations arounds its mean value. However, there might be information on the transport which can not be extracted from the noise. To get a full description of the transport processes through e.g. a quantum dot, a statistical description of the electronic transport is necessary. If it is possible to count individual electrons passing the device, analogously to cars passing a bridge, one can find the probability distribution of the number 
$N$ of electrons that have tunneled through the system during a certain time span. The full statistical characterization of the tunneling events is described by the probability distribution $P(N, t)$, which allows to calculate not just the mean and the variance of the number of transferred electrons, but in principle an infinite number of moments and cumulants. The knowledge of $P(N, t)$ constitutes a complete knowledge of the properties of the current fluctuations and is known as the full counting statistic (FCS) of the current.

FCS started as a theoretical investigation, developed by Levitov and Lesovik 26, 27 for non-interacting electrons in the scattering formalism and is a well established theoretical tool since two decades. Interaction effects have been included in subsequent works [100,101]. A widely used approach for Coulomb blockade systems based on a Markovian master equation has been introduced by Bagrets and Nazarov in 2003 [102]. More recent developments on the FCS focus for instance on the inclusion of non-Markovian dynamics 60, 63, 103 and finite-frequencies 40, 62, 104.

Nowdays there have been several experiments $28,58,59,61,105,114$ demonstrating that FCS is measureable. In 2006 S. Gustavsson et al. [28] realized the first experiment on counting statistics of single electron transport in a quantum dot using real-time electron detection techniques. Figure 2.6 shows the device and the result of the measurement presented in Ref. [28]. The device, Fig. 2.6 (a), consists of a quantum dot coupled to source and drain electrodes. A nearby quantum point contact is used as a charge detector to achieve real-time detection of single electron tunneling on and off the quantum dot. Gate electrodes tune the number of the electrons on the dot and the tunnel coupling strength to source and drain electrodes. Across the quantum dot a large bias voltage is applied to ensure that the electron transport is unidirectional. Figure 2.6 (b) displays the measured current through the quantum point contact as a function of real-time. This corresponds to the charge fluctuations of the quantum dot. If an electron jumps off the quantum dot the current increases and it decreases if another electron tunnels from the source on the dot. From the measurement of the charge 
Background and basic concepts

fluctuations on the quantum dot the statistical distribution of the number $n$ of electrons which enter the quantum dot during a given time period can be extracted. The statistical distribution is shown in Fig. 2.6 (c). The red solid line represents the theoretical prediction.

\subsection{Superconductivity, Proximity effect, An- dreev transport}

Since its discovery over 100 years ago, superconductivity is one of the most studied phenomena in physics. In $1911 \mathrm{H}$. Kamerlingh Onnes discovered that certain materials have zero electrical resistance when they are cooled down below a critical characteristic temperature [115]. This phenomenon, called superconductivity, was complemented by Meissner and Ochsenfeld in 1933 by the so-called Meissner Effect [116], the expulsion of a magnetic field from a superconductor during its transition to the superconducting state. In 1957 Bardeen, Cooper and Schrieffer proposed the well known BCS-theory [117, the first microscopic theory of superconductivity. By that time there was a quite complete theoretical picture of superconductivity. With the discovery of high temperature superconductors in 1986 by Bednorz and Müller 118 the field of superconductivity got revitalized. In the last decades the theoretical study of superconducting transport in nanoscale devices has experienced a great development [3]. With the introduction of mesoscopic physics a more detailed understanding of superconducting transport was developed around the central phenomenon of coherent Andreev reflection [11,119]. This concept opened the possibility to study the transport properties of different types of normal-superconductor structures and superconducting quantum point contacts as well as further aspects such as Andreev bound states or the Josephson effect. Nowdays, with the advances in nano fabrication, the realization of quantum dot superconductor hybrid structures has become feasible and attracted huge interest theoretically [3] as well as experimentally [2]. 
(a)

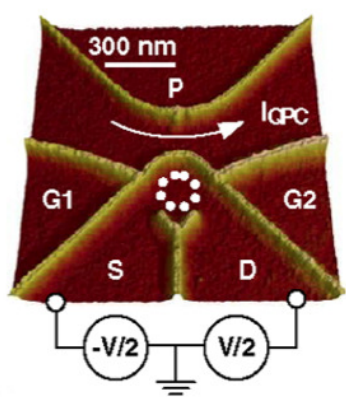

(c)

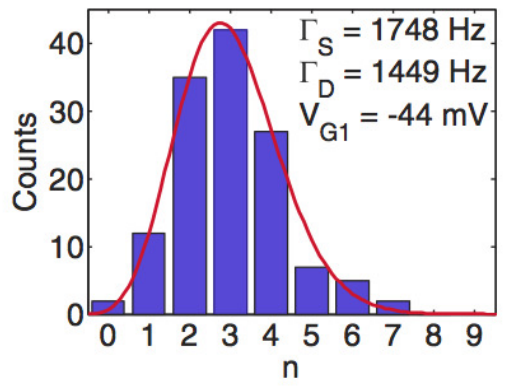

(b)
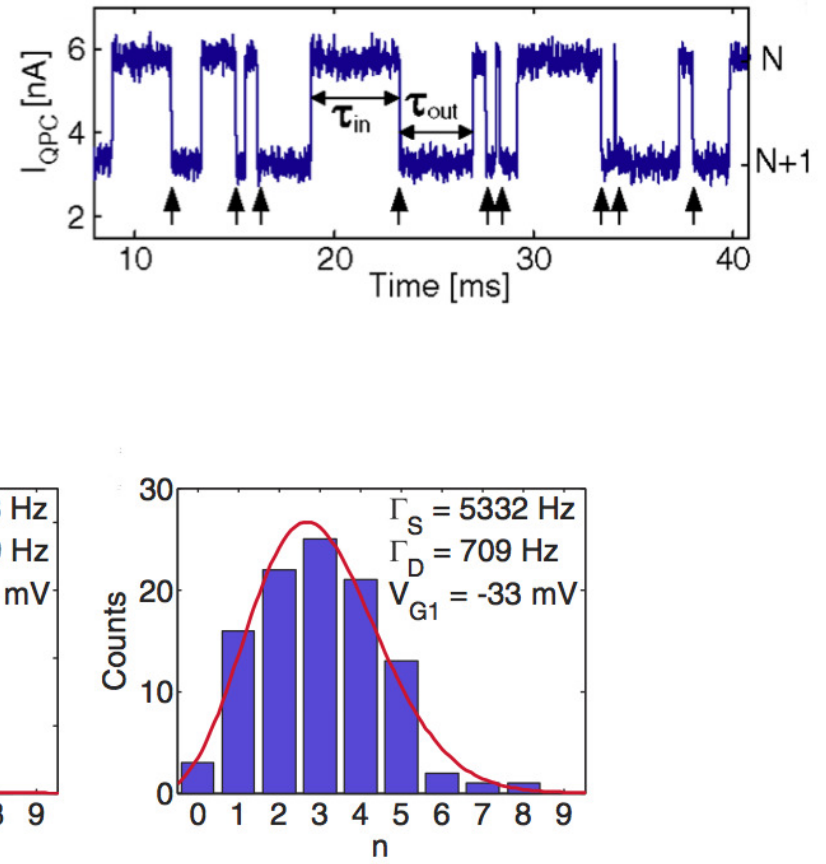

Figure 2.6: (a) Sample showing a semiconductor quantum dot coupled to source and drain electrodes. A nearby quantum point contact has the role to detect the charge fluctuations on the quantum dot. (b) Current measured through the quantum point contact as a function of time. (c) Statistical distribution of the number $n$ of electrons entering the dot during a given time. Each panel corresponds to a different value of the tunneling rate to source $\left(\Gamma_{\mathrm{S}}\right)$ and drain $\left(\Gamma_{\mathrm{D}}\right)$ electrode, obtained for different values of the gate voltage $\left(V_{\mathrm{G} 1}\right)$. Reprinted with permission from S. Gustavsson et al., Phys. Rev. Lett. 96, 076605, copyright (2006) by the American Physical Society. 
Background and basic concepts

\subsubsection{BCS-theory}

Superconductivity is one of the most studied phenomena of electronic correlations in low-energy condensed matter physics. The first microscopic theory of superconductivity was developed by Bardeen, Cooper and Schrieffer, known as BCS-theory [117]. Due to an attractive electron-phonon interaction Cooper pairs, consisting of two electrons with opposite momentum and spin, are formed. The pairing of the electrons into a boson-like state leads to an energy gap $\Delta$ in the spectrum of the superconductor. For energies below the gap (so-called subgap regime) transport is carried by Cooper pairs. To excite a quasiparticle from the ground state one needs at least the gap energy $\Delta$. If the gap $\Delta$ is larger than all other energy scales, only Cooper pairs are responsible for the transport properties of the superconductor. A pure supercurrent, namely a dissipationless current, will flow.

Within the scope of this thesis we are interested in the transport properties of a hybrid-superconductor-quantum dot structure. Hence, we need a microscopic theory to be able to calculate the transport properties. In the following we will briefly introduce the main concepts of the BCS-theory. For a more detailed introduction we refer to Ref. [120].

The first key result of the BCS-theory has been made by Cooper in 1956, showing that even a weak attraction can bind two electrons near the Fermi surface into a bound state [121]. In a metal two electrons close to the Fermi level with opposite spin and momentum combine into a so called Cooper pair caused by a weak attraction. The Fermi sea of electrons is unstable against the formation of at least one bound pair, regardless how weak the electronelectron interaction is, as long as it is attractive. This is the so called Cooper instability. Electrons condense to Cooper pairs until an equilibrium point is reached.

Considering the crucial ingredients of the BCS-theory as the attractive effective electron-electron interaction near the Fermi-surface and the Cooper pair instability of the Fermi sea, one can write the superconducting groundstate in terms of the following variational wave function 


$$
\left|\psi_{\mathrm{BCS}}\right\rangle=\prod_{\boldsymbol{k}}\left(u_{\boldsymbol{k}}+v_{\boldsymbol{k}} c_{\boldsymbol{k} \uparrow}^{\dagger} c_{-\boldsymbol{k} \downarrow}^{\dagger}\right)|0\rangle
$$

where $u_{\boldsymbol{k}}, v_{\boldsymbol{k}}$ are complex expansion coefficients and the index $\boldsymbol{k}$ denotes occupied states in momentum space. The BCS ground state is a superposition of states containing an integer number of Cooper pairs. The so-called BCS Hamiltonian is given by

$$
H_{\mathrm{BCS}}=\sum_{\boldsymbol{k}, \sigma} \xi_{\boldsymbol{k}} c_{\boldsymbol{k} \sigma}^{\dagger} c_{\boldsymbol{k} \sigma}+V \sum_{\boldsymbol{k} \boldsymbol{k}^{\prime}} c_{\boldsymbol{k}^{\prime} \uparrow}^{\dagger} c_{-\boldsymbol{k}^{\prime} \downarrow}^{\dagger} c_{\boldsymbol{k} \uparrow} c_{-\boldsymbol{k} \downarrow}+\text { constant. }
$$

where we already assumed that the pairing potential $V$ is independent of $\boldsymbol{k}, \boldsymbol{k}^{\prime}$, which is chosen to be $-V$ for states below a cut-off energy $\hbar \omega_{c}$ and zero otherwise. The second term of the BCS Hamiltonain Eq. (2.11) describes the pairing of two electrons with opposite spin and momenta into a singlet state, namely a Cooper pair, while the first term represents the quasiparticle excitations with its energy $\xi_{\boldsymbol{k}}$. Applying a mean-field treatment the BCS Hamiltonian Eq. (2.11) analogous to Hartree-Fock mean field theory yields,

$$
H_{\mathrm{BCS}}^{\mathrm{MF}}=\sum_{k, \sigma} \xi_{k} c_{\boldsymbol{k} \sigma}^{\dagger} c_{\boldsymbol{k} \sigma}-\Delta \sum_{\boldsymbol{k}} c_{\boldsymbol{k} \uparrow}^{\dagger} c_{-\boldsymbol{k} \downarrow}^{\dagger}-\Delta^{*} \sum_{\boldsymbol{k}} c_{\boldsymbol{k} \downarrow} c_{-\boldsymbol{k} \uparrow}
$$

with the pair potential $\Delta=V \sum_{\boldsymbol{k}^{\prime}}\left\langle c_{-\boldsymbol{k}^{\prime} \downarrow} c_{\boldsymbol{k}^{\prime} \uparrow}\right\rangle$. Note, that the mean field BCS Hamiltonian (Eq. 2.12) does not conserve the particle number. The Hamiltonian Eq. (2.12) can be diagonalized by a Bogoliubov transformation. The Bogoliubov transformation is defined by the following transformation of the creation and annihilation operators $c, c^{\dagger}$,

$$
\left(\begin{array}{c}
\gamma_{\boldsymbol{k} \uparrow} \\
\gamma_{-k \downarrow}^{\dagger}
\end{array}\right)=\left(\begin{array}{cc}
u_{k}^{*} & v_{k} \\
-v_{k}^{*} & u_{k}
\end{array}\right)\left(\begin{array}{c}
c_{k \uparrow} \\
c_{-k \downarrow}^{\dagger}
\end{array}\right)
$$

with the coefficents

$$
\left|u_{k}\right|^{2}=\frac{1}{2}\left(1+\frac{\xi_{k}}{E_{k}}\right) \quad \text { and } \quad\left|v_{k}\right|^{2}=\frac{1}{2}\left(1-\frac{\xi_{k}}{E_{k}}\right)
$$

and $E_{k}=\sqrt{\xi_{k}^{2}+|\Delta|^{2}}$. With this transformation we can obtain the following diagonalized Hamiltonain 


$$
H_{\mathrm{BCS}}^{\mathrm{MF}}=\sum_{\boldsymbol{k}} E_{\boldsymbol{k}}\left(\gamma_{\boldsymbol{k} \uparrow}^{\dagger} \gamma_{\boldsymbol{k} \uparrow}+\gamma_{\boldsymbol{k} \downarrow}^{\dagger} \gamma_{\boldsymbol{k} \downarrow}\right)
$$

with the new quasiparticle operators, the so-called bogoliubons, $\gamma_{\boldsymbol{k} \uparrow}, \gamma_{\boldsymbol{k} \downarrow}$ described by the number operator $\gamma_{\boldsymbol{k} \uparrow}^{\dagger} \gamma_{\boldsymbol{k} \uparrow}$.

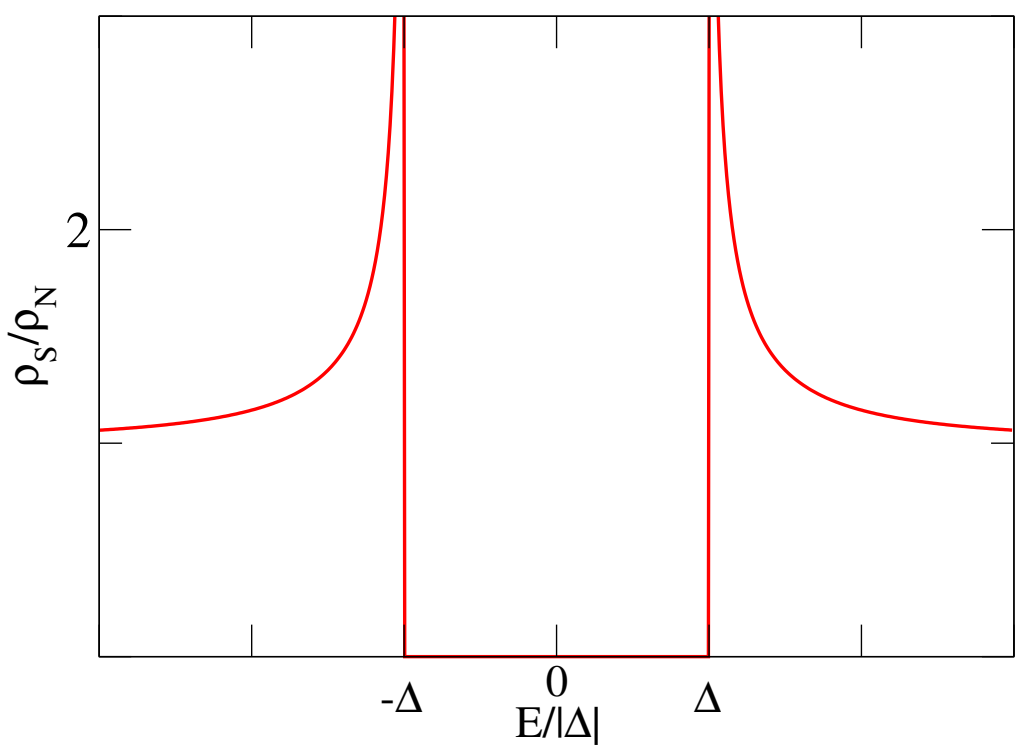

Figure 2.7: Density of states of the superconductor with a gap of $2|\Delta|$. Outside the gap quasiparticle excitations are possible.

The BCS quasiparticle density of states is shown in Fig. 2.7 with $\rho_{\mathrm{N}}$ the density of states of the normal state. The figure shows that the density of states has a gap of size $2|\Delta|$ around the Fermi level $\mu_{\mathrm{S}}$. This means there are no quasiparticle with an excitation energy within this gap and only Cooper pairs are allowed. For large energies $E \gg|\Delta|$ the density of states converges to the density of states of the normal state.

\subsubsection{Andreev reflection}

At the interface of a normal-conducting metal in contact with a superconductor, superconductivity can be induced in the normal metal. This phenomenon 
is known as proximity effect 122]. At the interface between superconductor and normal metal Cooper pairs can tunnel into the metal if the Fermi level of the normal metal lies within the gap of the superconductor. Superconductivity is induced in the normal metal and a gap will appear in the density of states of the normal-conducting metal.

If the energy $E$ of an electron is inside the gap $E<\Delta$ single particle tunneling between metal and superconductor is suppressed, since there are no quasiparticle states in the gap. For energies $E>\Delta$ quasiparticle transport is possible and single electrons can tunnel between the normal-conducting metal and the superconductor. At the interface between the normal-conducting metal and the superconductor an incoming electron will be reflected as a hole with opposite energy $E$ in the opposite direction retracing the original path of the electron. This mechanism, known as Andreev reflection [11], transfers Cooper pairs between a normal-conducting metal and a superconductor in a coherent way, i.e. the relation between the phase of the electron and the reflected hole is well defined and depends on the size of the superconducting gap $\Delta$. Each reflection process is related with a charge transfer of $2 e$.

In a superconductor - normal metal - superconductor (S-N-S) structure as depicted in Fig. 2.8 the reflected hole reaches the left superconductor and will be reflected as an electron with the energy $E^{\prime}$ with respect to the Fermi energy of the left superconductor. At each junction electrons and holes will be Andreev reflected, i.e. they cannot get out. The constructive interference of incident and reflected electron waves leads to discrete sub-gap states, the so-called Andreev bound states (ABS) [123].

Instead of a normal metal a quantum dot can be part of the superconducting junction. In that case the orbital levels of the quantum dot are relevant, rather than the Fermi level of the normal-conducting metal.

\subsubsection{Josephson effect}

The Josephson effect 124 was predicted by B.D. Josephson in 1962. A supercurrent flows without a bias voltage through the Josephson junction, the 


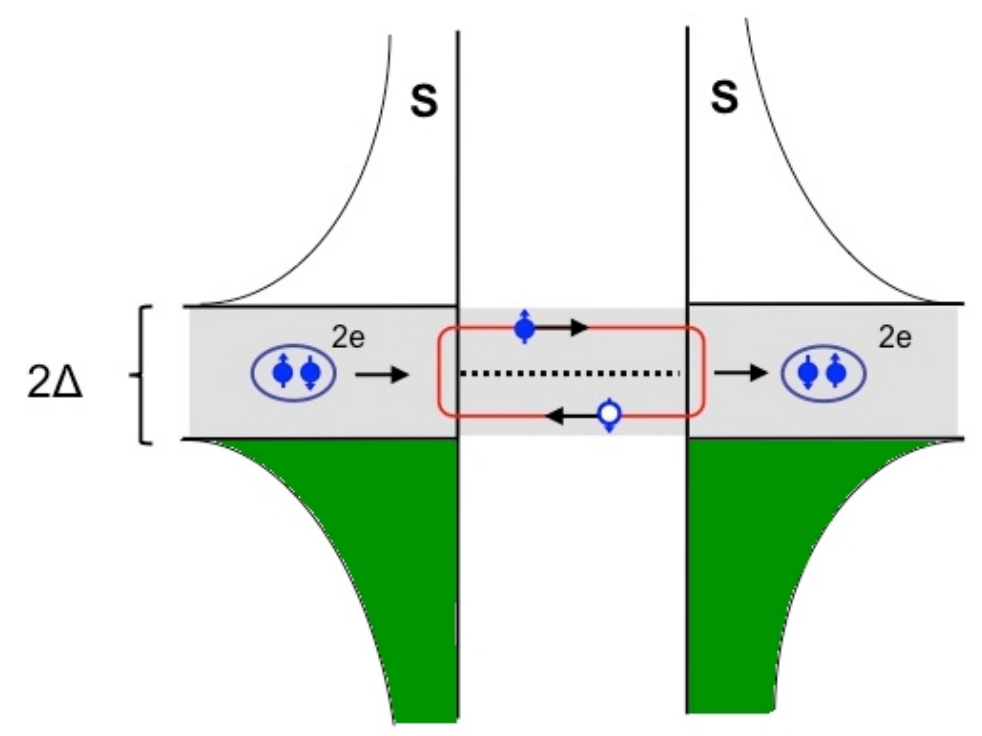

Figure 2.8: Schematic figure of a S-N-S structure. Electrons and holes get reflected at both junctions leading to Andreev bound states.

so called Josephson effect. A Josephson junction consists of two superconductors linked by a non-conducting barrier. A finite phase difference between the two order parameters of the superconductors $\phi=\varphi_{L}-\varphi_{R} \neq 0$ induces the flow of the so-called Josephson current.

Josephson predicted the following relations for voltage and current at zero bias

$$
\begin{aligned}
& J=J_{c} \sin \phi \\
& V=\frac{\hbar}{2 e} \frac{\partial \phi}{\partial t}
\end{aligned}
$$

The relations in (2.17) and (2.16) produce the following main effects:

1. The DC Josephson effect

This phenomena is given by the current-phase relation 2.16) and refers to a direct flow of the current through the insulator. An external current smaller than the critical current $I<J_{c}$ induces a phase difference between the two superconductor. Hence, a supercurrent due to the tunneling Cooper pairs is measurable through the device. If the external 
current is $I>J_{c}$ the Cooper pairs start to break up and also quasiparticles take part in the transport through the Josephson junction, hence the current becomes dissipative.

2. The AC Josephson effect

If a constant voltage is applied across the junction, then the phase will vary linearly in time and the current through the device will be an $\mathrm{AC}$ current with a frequency proportional to the applied voltage as shown in (2.17). The AC Josephson effect is a perfect voltage to frequency converter.

As mentioned above the phase difference of the two superconductors induces the supercurrent $J=J_{c} \sin \phi$. There exists also a second realization of the DC Josephson effect, namely $J=J_{c} \sin (\phi+\pi)=-J_{c} \sin \phi$. It is obtained by a Josephson junction, where the critical current is negative $J_{c}<0$. Such a Josephson junction is called a $\pi$ junction. The change of the sign in the critical current implies that for a given phase difference, the supercurrent through a $\pi$ junction is reversed with respect to an ordinary Josephson junction (0-junction).

For instance, a $\pi$ junction behavior can occur in a Josephson junction with a ferromagnetic Josephson barrier, i.e. a superconductor-ferromagnetsuperconductor (SFS) junction. In the ferromagnetic layer the superconducting order parameter oscillates in the direction perpendicular to the junction plane. Depending on the thickness of the ferromagnetic layer one can obtain a $\pi$ Josephson junction. Various ferromagnetic $\pi$ junctions have been fabricated 125128 .

The Josephson effect has found wide usage of application in several areas. The most famous ones are probably SQUIDs (Superconducting Quantum Interference Devices), used as ultra-sensitive magnetometers that operate via the Josephson effect. 


\subsection{Quantum dots coupled to superconduc- tors}

Instead of a normal-conducting lead a quantum dot can be part of the superconducting junction and hence, superconducting correlations can be induced on quantum dots. Hybrid devices with quantum dots open the possibility to control single electrons (quantum dots) in the presence of a macroscopic quantum phenomenon involving large numbers of electrons (superconductivity). These nanoscale structures are an ideal playground to study superconducting correlations in systems with few degrees of freedom that exhibit strong Coulomb-interaction effects. A variety of hybrid-superconductorquantum dot setups have been the scope of different theoretical [3] as well as experimental studies [2]. In this section we will review some of the prominent experimental achievements demonstrating the relevance of these systems.

In the last decades with the advances in nano fabrication techniques, it became possible to couple quantum dots to superconductors $9,10,12,21,129$ 139] and to study superconducting correlations in these devices. The first experimental evidence of superconducting properties in transport through a hybrid superconductor-quantum dot device has been reported in 1995 [140]. The experiment showed a signature of the superconducting gap and provided evidence of a modified quasiparticle density of states near the gap. However, it was not possible to measure a supercurrent, namely a Josephson current, because the measurement could only be performed in a transport regime where quasiparticle tunneling is the dominant transport mechanism. The difficulty was for a long time that due to technical reasons, it was not possible to enter a regime where Cooper pairs tunnel resonantly across the device leading to a supercurrent. For instance, oxidation at the interface between superconductors and conventional semiconductors suppresses the tunneling of Cooper pairs. Nevertheless with the introduction of new materials for quantum dots, like carbon nanotubes or semiconducting nanowires, the evidence of a supercurrent became accessible due to strong coupling favoring 
multi particle transport.

The first measurement of a supercurrent in hybrid-superconductor quantum dot device was realised in 2006 by P. Jarillo-Herrero et al., Ref. 133. The quantum dot was created in a single-walled carbon nanotube with aluminum based superconducting leads. The advantage of carbon nanotubes is their large energy level spacing, which allows for a resonant tunneling of Cooper pairs across the device. Whenever a dot level is aligned with the Fermi energy of the superconductors, a resonant supercurrent can flow and the critical current is maximal. In contrast, the critical current gets suppressed whenever the Fermi energy lies between two dot levels. Hence, the critical current as a function of the applied gate voltage shows resonance peaks every time a level is aligned with the Fermi energy and gets suppressed elsewhere.

In a subsequent work by van Dam et al. Ref. [139, they show a first measurement of a supercurrent in a transport regime where resonant tunneling of Cooper pairs is prohibited owing to Coulomb blockade. Even though a strong Coulomb repulsion on the quantum dot does not allow a simultaneous occupation of the dot and hence a resonant tunneling of Cooper pairs is suppressed, a supercurrent can still flow due to a subsequent coherent transport of correlated electrons. Figure 2.9 shows the superconductor-quantum dot device and the resulting supercurrent. The superconductor quantum dot device is formed in a superconducting interference device (SQUID), Fig. 2.9 (a). The Josephson current is flowing from one superconducting junction through a strongly interacting quantum dot into another superconductor. Each superconducting arm contains a break which is bridged by an InAs nanowire. Two local gate electrodes are used to define the quantum dot in the top nanowire. This allows to obtain a tunable coupling to the superconducting leads. The phase difference between the superconducting arms is controlled by a magnetic flux in the SQUID. The other nanowire defines a superconducting weak link, with just one gate to control the strength of the Josephson coupling. Figure 2.9 (b) shows the critical current of the quantum dot as a 
(a)
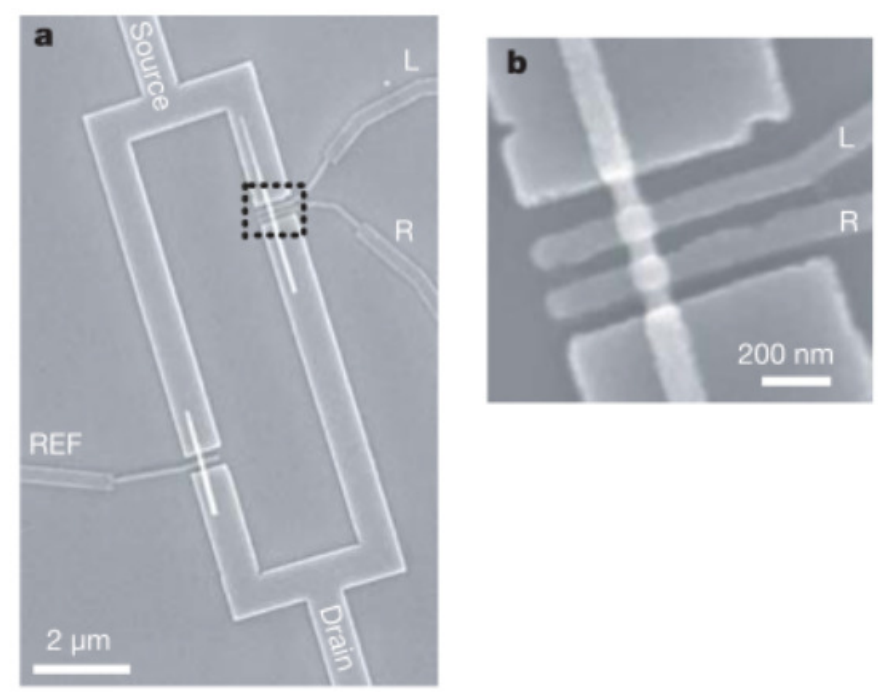

(b)

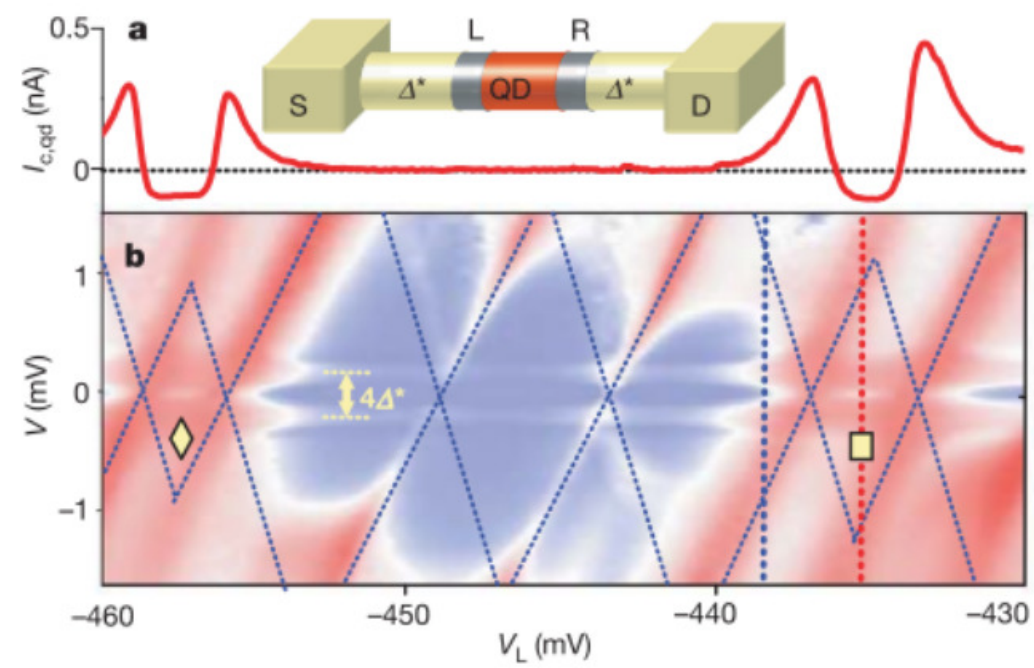

Figure 2.9: (a) Scanning electron microscope image of a quantum dot coupled to superconducting leads. (b) (top) Critical supercurrent of the quantum dot as a function of the gate voltage and (bottom) density plot of the differential conductance of the gate and bias voltage. Reprinted by permission from Macmillan Publishers Ltd: Nature 442, 667, copyright 2006. 
function of the gate voltage (top) and the corresponding differential conductance (bottom). The critical supercurrent can be observed in Fig. 2.9 (b). The sign change is due to the fact that the supercurrent is caused by coherent tunneling of single electrons. Whenever transport occurs through a single spin-degenerate level filled with one electron, the spin-ordering of the Cooper pair gets reversed during the transport event. This results in a sign change in the Cooper pair singlet and hence, gives a negative contribution to the supercurrent. Changing the occupation of the quantum dot from a even to an odd occupation by adding single electrons on the quantum dot results in a transition from a positive to a negative critical current, a so-called $0-\pi$ transition.

The supercurrent which flows through a hybrid superconductor-quantum dot device is carried by its ABSs, which form when a normal-conducting region, here a quantum dot, is in contact to a superconductor. In various experimental studies the subgap structure in superconductor-quantum dotsuperconductor and superconductor-quantum dot- normal metal has been analyzed by Andreev level spectroscopy [12 22], which permits measurement of the Andreev addition energies via the differential conductance. Figure 2.10 shows the device and a result of the first measurement detecting the presence of ABS [15] in the proximzed dot spectrum which clearly modify the physical properties of the quantum dot. As displayed in Fig. 2.10 (a) the quantum dot in the first tunneling spectroscopy experiment of individually resolved ABS has been realized in a carbon nanotube coupled to two superconducting leads, while a third electrode acts as a tunnel probe. The Andreev level spectroscopy reveals information about the addition energies, usually called Andreev levels and the total line width of the resonances. The density of states of the carbon nanotube, Fig. 2.10 (b), can be extracted form the differential conductance of the tunnel probe. The excitation energies are bound on the interval $\pm \Delta$ and clearly differ from the usual Coulomb diamond pattern as known from the normal state spectroscopy (see Sec. 2.3).

Figure 2.11 shows a recent experiment on a hybrid superconductor- quan- 
(a)
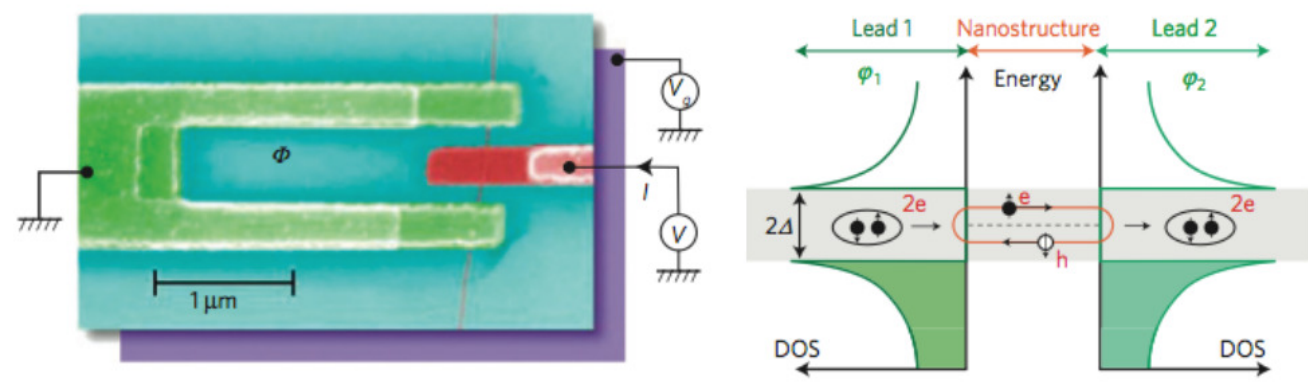

(b)

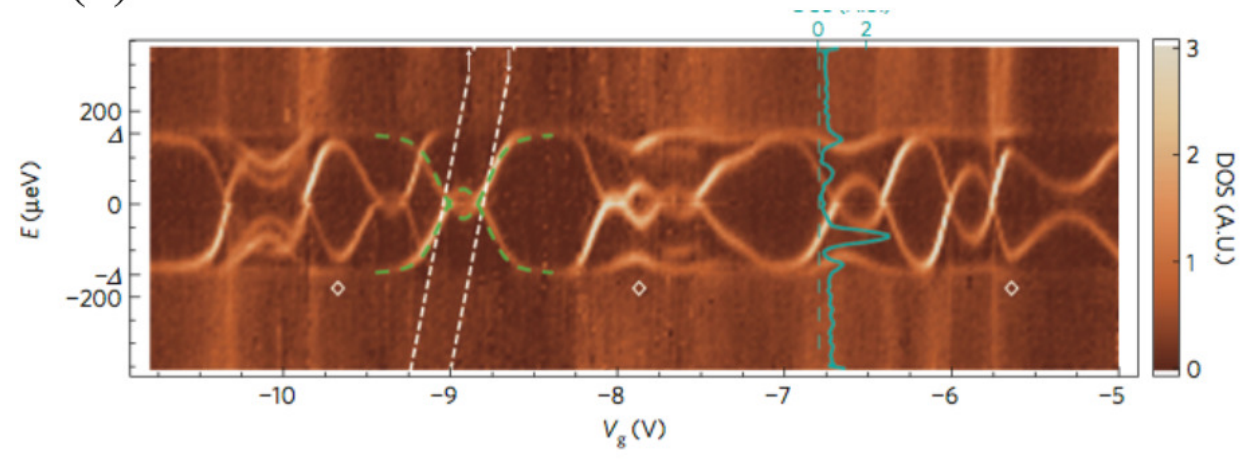

Figure 2.10: (a) Scanning electron micrograph of the device used for ABS spectroscopy. A carbon nanotube (thin vertical line) is connected to two superconducting contacts (green) with a weakly coupled tunnel electrode (red) in between. The substrate (purple) acts as a backgate. Schematic figure on the right shows the formation of the ABS in the system. (b) Result of the measurement of the density of states in the carbon nanotube. Reprinted by permission from Macmillan Publishers Ltd: Nature Phys. 6, 965, copyright 2010. 
tum dot device by E. Lee et al. 20. In particular Fig. 2.11 (a) shows the realization of a quantum dot strongly coupled to a superconductor and weakly coupled to a normal-conducting lead. The device contains a single shell nanowire, where the quantum dot is naturally formed due to the device geometry between the superconducting and normal contact. The quantum dot formed on the nanowire is tuned by means of three gates. Two bottom electrodes, namely a plunger gate and a barrier gate close to the superconducting electrode, are used to control the charge on the quantum dot and to control the coupling strength to the superconductor. The third gate is a back gate that is used to fine tune the tunnel coupling. The current in such a system for bias voltages smaller than the superconducting gap $\Delta$ is carried by Andreev reflection processes. Hence, the result of the tunnel spectroscopy in terms of the differential conductance (Fig. 2.11(b)) detects the subgap excitations of the system, namely the Andreev levels.

Hybrid superconductor-normal structures offer a wide spectrum for novel devices and applications. For instance quantum dot devices coupled to two superconducting electrodes are similar to Josephson junctions. Superconducting quantum bits, the basic unit of quantum computing, based on Josephson junctions have been demonstrated in Ref. 141. Also, ABS are proposed to be used as quantum bits [142]. Double quantum dots, tunnel coupled to superconductors have been used as a source of entangled electron pairs $[9,10]$. This could lead to further applications with theses types of devices to exploit quantum entanglement and to study fundamental aspects of quantum mechanics in general. Currently, there is a huge research interest in the creation and manipulation of Majorana fermions, particles which are their own antiparticles, in solid-state device [143]. Also hybrid superconductor-quantum dot devices are at the heart of recent proposals to generate Majorana-fermion excitations in quantum dots 44 7]. In conclusion, these points demonstrate the huge research effort in the field of hybrid normal-superconductor nanodevices and also its importance for the development of new types of devices for a range of applications. Hence, it is of 
vital importance to get access to the properties of such hybrid quantum-dot systems. 
(a)
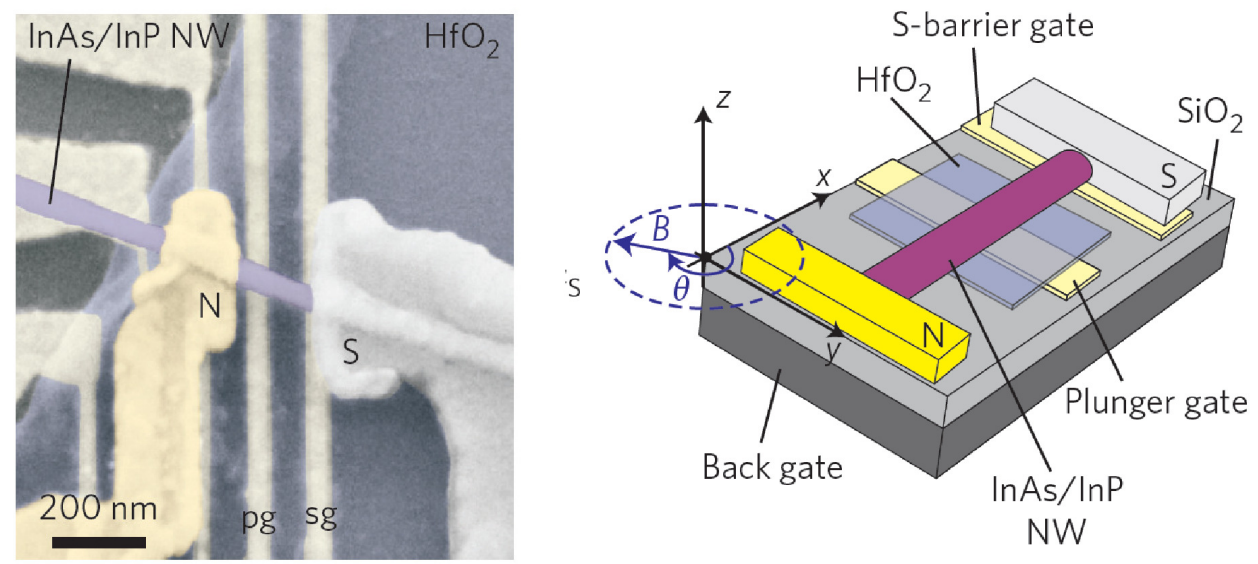

(b)

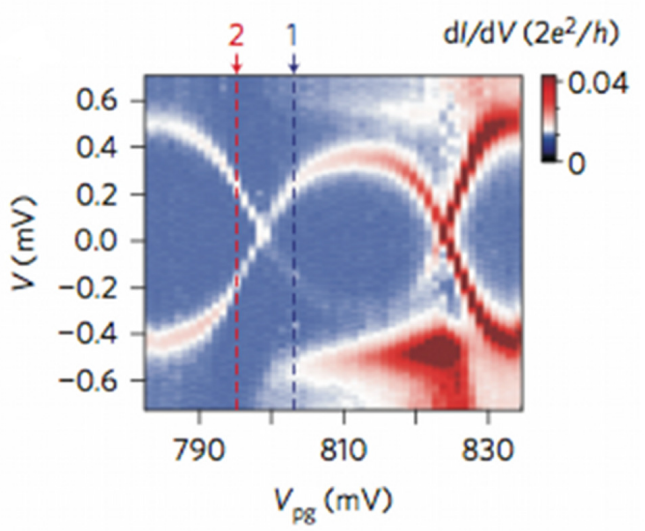

Figure 2.11: (a) (right) Scanning electron micrograph of the quantum dot device coupled a superconductor and a normal-conducting reservoir and (left) schematic picture of the device. (b) Density plot of the differential conductance with clearly resolves the subgap excitations, namely the Andreev levels. Reprinted by permission from Macmillan Publishers Ltd: Nature Nanotechnology 9, 79, copyright 2013. 


\section{Chapter 3}

\section{Diagrammatic real-time approach}

In this thesis we study the current fluctuations in different setups of singlelevel quantum dots coupled to electronic reservoirs, in particular normal and superconducting leads. We take into account on-site Coulomb interaction of arbitrary magnitude and non-equilibrium conditions, without resorting to the linear-response regime. While we are interested in a strong coupling of the quantum dot to the superconducting lead, leading to strong superconducting correlations, we assume the coupling to the normal-conducting lead to be weak. Considering these conditions, we make use of a diagrammatic realtime perturbation theory in the tunnel-coupling with the normal lead 64 . 65] and its extension to a system with superconducting electrodes [49], in order to derive the current, the finite-frequency current noise and the finitetime FCS. The formalism to obtain the finite-frequency noise using the realtime diagrammatic approach has been introduced previously, for the case of normal-conducting electrodes 144 as well as for the ferromagnetic case 34].

In this chapter we introduce the theory to obtain the current, finitefrequency noise and the finite-time FCS for non-equilibrium transport through a strongly interacting quantum dot making use of the diagrammatic real-time approach. 
The diagrammatic-real time transport theory [64 66] is a general transport theory used to describe non-equilibrium transport through a strongly interacting quantum dot weakly coupled to electronic reservoirs. The basic idea of the theory is to split the system into non-interacting regions with many degrees of freedom (the electronic reservoirs) and a strongly interacting region (here a quantum dot) that contains only a small number of degrees of freedom and to integrate out all reservoir degrees of freedom by means of Wick's theorem. The system is described in terms of a reduced density matrix. The advantage of the method is that the on-site Coulomb interaction on the dot can be taken into account exactly.

This chapter is organized as follows: In Sec. 3.1 we introduce the Hamiltonian of the system. In Sec. 3.2 we derive a master equation to determine the reduced density matrix of the quantum dot. In the subsequent sections, we present a diagrammatic representation to determine the current, Sec. 3.3 and the finite-frequency noise, Sec. 3.4. Next, in Sec. 3.5, we present a theory to obtain the finite-time FCS for a non-Markovian master equation. In the last section of this Chapter, Sec. 3.6, we extend the theory presented in Sec. 3.5 and introduce the multi-time FCS for Markovian processes.

\subsection{Model}

We consider a single-level quantum dot tunnel-coupled to normal and superconducting leads. Figure 3.1 shows a sketch of a single-level quantum dot coupled to normal-conducting leads, Fig. 3.1 (a) and one normal and one superconducting lead, Fig. 3.1(b). The Hamiltonian contains three terms, $H=H_{\mathrm{D}}+\sum_{\eta=\mathrm{S}, \mathrm{N}}\left(H_{\eta}+H_{\text {tunn }, \eta}\right)$, where $\eta=\mathrm{N}$, S labels the normal $(\mathrm{N})$ and superconducting $(\mathrm{S})$ lead. When considering normal-conducting leads only, as shown in Fig. 3.1 (a), we use the notation $\eta=\mathrm{L}, \mathrm{R}$ for left and right reservoir. The quantum dot is described by the Anderson impurity model with a spin-degenerate level with energy $\epsilon$ and the on-site Coulomb repulsion 
(a)

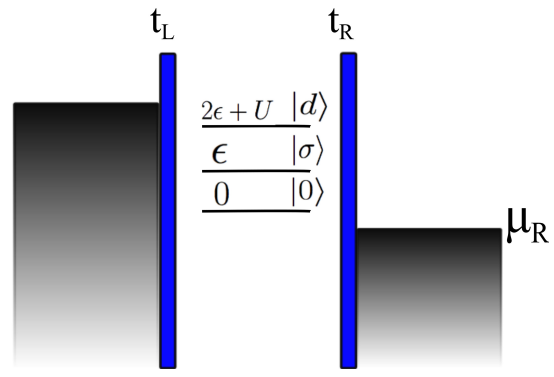

(b)

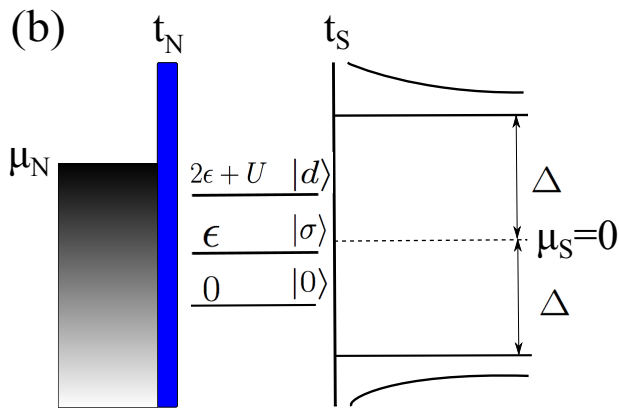

Figure 3.1: (a) Sketch of a single-level quantum dot with energy level $\epsilon$ and on-site Coulomb interaction $U$ coupled to normal-conducting leads $\left(t_{\mathrm{L}}\right.$ and $t_{\mathrm{R}}$ ). The chemical potential of the leads is given by $\mu_{\eta}$ with $\eta=\mathrm{L}, \mathrm{R}$ (b) Sketch of a single-level quantum dot with energy level $\epsilon$ and on-site Coulomb interaction $U$, tunnel coupled to one normal lead $\left(t_{\mathrm{N}}\right)$ and one superconducting lead $\left(t_{\mathrm{S}}\right)$.

$U$,

$$
H_{\mathrm{D}}=\sum_{\sigma} \epsilon \hat{n}_{\sigma}+U n_{\uparrow} n_{\downarrow}
$$

Here, $\hat{n}_{\sigma}=d_{\sigma}^{\dagger} d_{\sigma}$ is the occupation number of electrons with spin $\sigma$ on the dot and $d_{\sigma}\left(d_{\sigma}^{\dagger}\right)$ is the annihilation (creation) operator for an electron with spin $\sigma=\uparrow, \downarrow$. The isolated quantum dot has the following eigenstates: $|0\rangle$ (empty), $|\sigma\rangle=d_{\sigma}^{\dagger}|0\rangle$ (singly occupied) and $|d\rangle=d_{\uparrow}^{\dagger} d_{\downarrow}^{\dagger}|0\rangle$ (doubly occupied). The Hamiltonian of the lead $\eta=\mathrm{N}$, S reads

$$
H_{\eta}=\sum_{k, \sigma} \epsilon_{\eta k} c_{\eta k \sigma}^{\dagger} c_{\eta k \sigma}-\delta_{\eta, \mathrm{S}} \Delta \sum_{k}\left(c_{\eta-k \downarrow} c_{\eta k \uparrow}+\text { H.c. }\right)
$$

where $c_{\eta k \sigma}\left(c_{\eta k \sigma}^{\dagger}\right)$ are the lead-electron operators with $k$ being the wave vector in lead $\eta$. We have $\delta_{\eta, \mathrm{S}}=0$ for the normal conducting lead and $\delta_{\eta, \mathrm{S}}=1$ for the superconducting lead; $\Delta$ is the superconducting gap. We choose the electrochemical potential of the superconductor as a reference, $\mu_{\mathrm{S}}=0$. The normal-conducting lead has an electrochemical potential $\mu_{\mathrm{N}}$, which in general differs from zero. For a quantum dot coupled to two normal leads, 
as displayed in Fig. 3.1(a), we have to consider the electrochemical potential of left $\left(\mu_{\mathrm{L}}\right)$ and right reservoir $\left(\mu_{\mathrm{R}}\right)$ and we assume a symmetric applied bias voltage across the device, $\mu_{\mathrm{L}}=-\mu_{\mathrm{R}}=V / 2$, where we have set $e=1$.

The tunneling between the dot and the leads is modeled by the tunneling Hamiltonians

$$
H_{\mathrm{tunn}, \eta}=t_{\eta} \sum_{k, \sigma} c_{\eta k \sigma}^{\dagger} d_{\sigma}+\text { H.c. }
$$

We assume the normal conducting density of states $\rho_{\eta}$ of the leads to be constant and $t_{\eta}$ to be spin- and momentum independent and define the tunnelcoupling strength as $\Gamma_{\eta}=2 \pi \rho_{\eta}\left|t_{\eta}\right|$.

\subsubsection{Effective Hamiltonian}

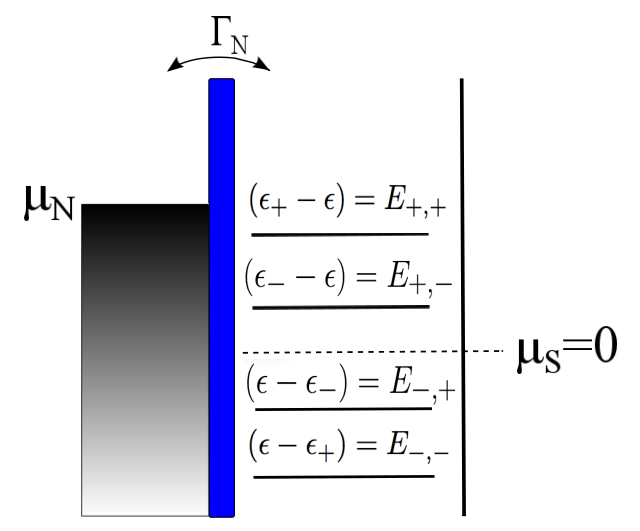

Figure 3.2: Sketch of the effective dot-superconductor subsystem coupled to a normal conducting lead $\left(\Gamma_{\mathrm{N}}\right)$ which acts as a bath.

For a quantum dot coupled to superconducting leads we consider the limit $\Delta \rightarrow \infty$, since we are interested in the subgap features of transport. Quasiparticle transport to the superconductor is suppressed and the effect of the superconductor can be understood in terms of the proximity effect. This effect can be cast in an effective Hamiltonian 145,

$$
H_{\mathrm{eff}}=H_{\mathrm{D}}-\frac{\Gamma_{\mathrm{S}}}{2}\left(d_{\uparrow}^{\dagger} d_{\downarrow}^{\dagger}+d_{\downarrow} d_{\uparrow}\right)
$$


which includes the coupling between quantum dot and the superconducting lead and describes the pairing on the dot induced by the proximity effect. The eigenstates of the isolated quantum dot hence differ from the eigenstates of the effective Hamiltonian. The effective dot-superconductor subsystem coupled to a normal conducting lead is sketched in Fig. 3.2. Due to the tunnel coupling to the superconductor the states $|0\rangle$ and $|d\rangle$ combine to new eigenstates, the Andreev bound states (ABS),

$$
| \pm\rangle=\frac{1}{\sqrt{2}} \sqrt{1 \mp \frac{\delta}{2 \epsilon_{\mathrm{A}}}}|0\rangle \mp \frac{1}{\sqrt{2}} \sqrt{1 \pm \frac{\delta}{2 \epsilon_{\mathrm{A}}}}|d\rangle,
$$

with the energies $\epsilon_{ \pm}=\delta / 2 \pm \epsilon_{\mathrm{A}}$, the detuning $\delta=2 \epsilon+U$ between the empty and the double occupied state and the splitting between the $|+\rangle$ and $|-\rangle$ states, $2 \epsilon_{\mathrm{A}}=\sqrt{\delta^{2}+\Gamma_{\mathrm{S}}^{2}}$. The superconducting correlation on the dot is strongest, if the mixing between the empty $|0\rangle$ and doubly occupied state $|d\rangle$ is maximal. This is the case for $\delta \sim 0$, namely when the states $|0\rangle$ and $|d\rangle$ are nearly degenerate (the proximity effect is on resonance). The excitation energies of the dot are the so called Andreev addition energies, which are given by the differences of the eigenenergies of the states whose occupation numbers differ by one

$$
E_{\gamma^{\prime}, \gamma}=\gamma^{\prime} \frac{U}{2}+\gamma \epsilon_{\mathrm{A}}
$$

with $\gamma^{\prime}, \gamma= \pm 1$. The energy of the electron leaving or entering the normal lead must account for the energy difference between the initial and final state of the dot-superconductor subsystem, which are the Andreev addition energies, represented by the energy levels sketched in Fig. 3.2

At this stage it is useful to introduce effective coupling strengths which describe the coupling between the electronic reservoir and the dot resonances, namely the Andreev levels. These effective coupling strengths turn out to be essential to understand the steps occurring in the finite-frequency noise due to quantum fluctuations at frequencies equal to the internal energies of the quantum dot.

The effective tunnel-coupling strengths of the Andreev levels to the normal conducting lead $\Gamma_{\mathrm{N}}$ corresponding to the excitation $|\sigma\rangle$ to $| \pm\rangle$ are given 
by

$$
\Gamma_{\sigma \rightarrow \pm}=\frac{\Gamma_{\mathrm{N}}}{2}\left(1 \pm \frac{\delta}{2 \epsilon_{\mathrm{A}}}\right)
$$

while the Andreev levels of the excitation from $| \pm\rangle$ to $|\sigma\rangle$ have the effective coupling strength

$$
\Gamma_{ \pm \rightarrow \sigma}=\frac{\Gamma_{\mathrm{N}}}{2}\left(1 \mp \frac{\delta}{2 \epsilon_{\mathrm{A}}}\right) .
$$

Throughout the text we use $\hbar=e=1$.

\subsection{Master equation}

In this section we present the theory which allows us to investigate electronic transport through a quantum dot coupled to normal and superconducting leads. Since we are only interested in the dynamics of the strongly interacting quantum dot, we integrate out the electronic reservoir degrees of freedom. In the following we derive a master equation, that describes the time evolution of the density matrix of the system based on the previously introduced model. In order to solve the master equation we perform a perturbation expansion up to first order in the tunnel-coupling strength to the normal conducting lead. This allows us to take the Coulomb interaction on the quantum dot into account exactly.

\subsubsection{Density matrix}

All dynamics of the system can be identified by the time evolution of the density matrix. Each observable can be determined by the expectation value of the respective operator. The quantum mechanical expectation value of an operator $A$ at some time $t$ in terms of the initial density matrix $\rho\left(t_{0}\right)$ in the Heisenberg representation is given by

$$
\langle A\rangle(t)=\operatorname{Tr}\left[A(t)_{H} \rho\left(t_{0}\right)\right]
$$

with the initial time $t_{0}$. Assume that at some initial time $t_{0}$, the tunnel coupling between the quantum dot and the electronic reservoir is switched 
on. Hence, the tunneling Hamiltonian vanishes for $t \leq t_{0}$ and the quantum dot is decoupled from the reservoirs. In this case the initial density matrix $\rho\left(t_{0}\right)$ can be factorized into parts of the quantum dot and the lead electrons,

$$
\rho\left(t_{0}\right)=\rho_{\text {red }}\left(t_{0}\right) \otimes \rho_{\text {res }}\left(t_{0}\right)
$$

where $\rho_{\text {res }}\left(t_{0}\right)=Z_{\text {res }}^{-1} \prod_{\eta} \exp \left(-\beta_{\eta}\left(\mathrm{H}_{\eta}-\mu_{\eta} \hat{\mathrm{N}}_{\eta}\right)\right)$ is the equilibrium density matrix of the reservoir with the inverse temperature $\beta=1 / k_{B} T$, the electrochemical potential $\mu_{\eta}$ and the number operator of the electrons in lead $\eta$, $\hat{N}_{\eta}=\sum_{k \eta} c_{\eta k \sigma}^{\dagger} c_{\eta k \sigma}$. The normalization factor $Z_{\text {res }}$ can be determined by fulfilling the condition $\operatorname{Tr} \rho_{\text {res }}\left(t_{0}\right)=1$. The degrees of freedom of the quantum dot are described in terms of a reduced density matrix, which is defined as

$$
\boldsymbol{P}=\rho_{\text {red }}=\operatorname{Tr}_{\text {res }}(\rho)
$$

where the trace over the non-interacting reservoir degrees of freedom is denoted by $\operatorname{Tr}_{\text {res }}$. Since we are not interested in the degrees of freedom of the reservoir we trace them out, making use of Wick's theorem. More details are discussed in the following. The advantage of the reduced system is that we are left with an effective description of the strongly interacting quantum dot.

The elements of the reduced density matrix are defined by $P_{\chi}^{\chi^{\prime}}=\left\langle\chi^{\prime}|\boldsymbol{P}| \chi\right\rangle$, where the kets $|\chi\rangle,\left|\chi^{\prime}\right\rangle$ are the quantum dot states. The diagonal elements of the reduced density matrix $P_{\chi}^{\chi}=P_{\chi}$ are the probabilities to find the quantum dot in state $|\chi\rangle$. They fulfill the normalization condition $\sum_{\chi} P_{\chi}=1$. Off-diagonal elements in the reduced density matrix, which we refer to as the coherences, may arise due to the coupling of the quantum dot to the electronic reservoirs.

\subsubsection{Generalized master equation}

Our aim is to calculate the transport properties of a strongly interacting quantum dot out of equilibrium. This problem does not have a known exact solution. We are interested in the non-equilibrium time evolution of the 
reduced density matrix. We choose to perform a perturbation expansion in the tunnel coupling (with the tunneling Hamiltonian as a perturbation) between the strongly interacting quantum dot and the electronic reservoirs. It is now useful to switch to the interaction picture and to perform the perturbation expansion formulated in Keldysh time [146], since we want to treat a strongly interacting system out of equilibrium. The non-equilibrium time evolution of the reduced density matrix can be depicted on the Keldysh contour and expressed in terms of a propagator $\boldsymbol{P}(t)=\boldsymbol{\Pi}\left(t, t^{\prime}\right) \boldsymbol{P}\left(t^{\prime}\right)$. An example of the Keldysh contour for the calculation of the current is shown in the sketch in Fig. 3.3 (a). The upper (lower) horizontal time line stands for the propagation of the individual dot state forward (backward) in realtime, indicated by arrows. The terms of the tunneling Hamiltonian Eq. (3.3) are represented by vertices on the Keldysh contour. Each vertex describes transport of one electron from the quantum dot to the reservoir and vice versa. Tunneling lines between two vertices take into account contractions between two lead creation and annihilation operators by means of Wicks's theorem. More details on how to calculate the diagrams are summarized in the following. To formulate propagation along the Keldysh contour in a more compact form it is convenient to introduce a time ordering operator $T_{K}$ which orders all following operators with respect to the Keldysh contour time.

We derive now a time-dependent master equation that describes the nonequilibrium time evolution of all reduced density matrix elements. Therefore we need to calculate the expectation value of the projection operator of the dot states and find for the non-equilibrium density matrix elements

$$
P_{\chi_{2}}^{\chi_{1}}=\left\langle\mid \chi_{2}\right\rangle\left\langle\chi_{1} \mid(t)\right\rangle=\operatorname{Tr}\left[T_{K} \exp \left(-i \int_{K} d t^{\prime} H_{\text {tunn }}\left(t^{\prime}\right)_{I}\right)\left|\chi_{2}\right\rangle\left\langle\chi_{1}\right|(t) \rho\left(t_{0}\right)\right]
$$

Using Eq. (3.10) and writing explicitly the trace over the states of the quan- 


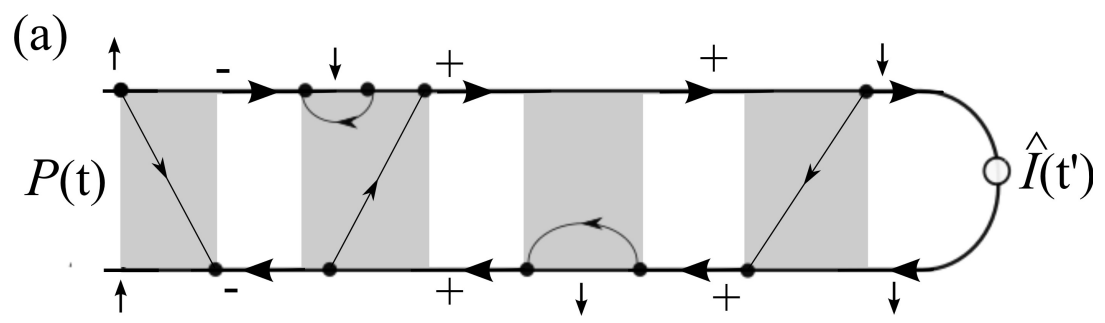

(b)

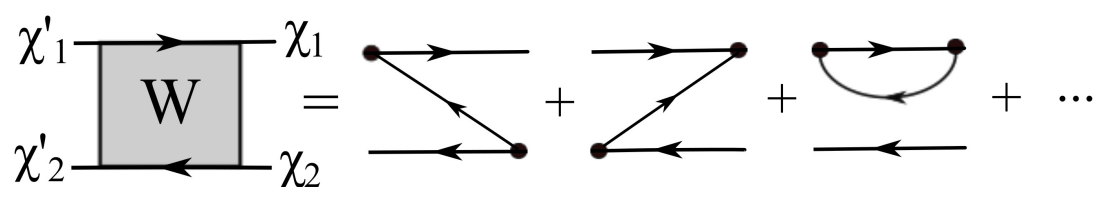

Figure 3.3: (a) Example of the time evolution of the reduced density matrix $\mathbf{P}(t)$ for the evaluation of the expectation value of the current in case of a quantum dot coupled to a normal and superconducting lead. The reduced system propagates forward in time along the top path from $t$ to $t^{\prime}$ at which the observable $I$ is measured, then the system propagates back to time $t$. (b) Diagrammatic representation of the matrix element $W_{\chi_{2} \chi_{2}^{\prime}}^{\chi_{1} \chi_{1}^{\prime}}$. 
tum dot the matrix elements of the reduced density matrix read,

$$
\begin{aligned}
P_{\chi_{2}}^{\chi_{1}}= & \sum_{\chi_{1}^{\prime}, \chi_{2}^{\prime}}\left\langle\chi_{2}^{\prime}\right| \operatorname{Tr}_{\text {leads }}\left[T_{K} \exp \left(-i \int_{K} d t^{\prime} H_{\text {tunn }}\left(t^{\prime}\right)_{I}\right)\left|\chi_{2}\right\rangle\left\langle\chi_{1}\right|(t) \rho_{\text {res }}\left(t_{0}\right)\right] \times \\
& \left|\chi_{1}^{\prime}\right\rangle P_{\chi_{2}^{\prime}}^{\chi_{1}^{\prime}}\left(t_{0}\right) .
\end{aligned}
$$

Now, we are able to introduce the full propagator of the reduced system as

$$
\begin{aligned}
\Pi_{\chi_{2} \chi_{2}^{\prime}}^{\chi_{1} \chi_{1}^{\prime}}\left(t, t_{0}\right)= & \left\langle\chi_{2}^{\prime}\right| \operatorname{Tr}_{\text {leads }}\left[T_{K} \exp \left(-i \int_{K} d t^{\prime} H_{\text {tunn }}\left(t^{\prime}\right)_{I}\right) \times\right. \\
& \left.\left|\chi_{2}\right\rangle\left\langle\chi_{1}\right|(t) \rho_{\text {res }}\left(t_{0}\right)\right]\left|\chi_{1}^{\prime}\right\rangle .
\end{aligned}
$$

In terms of the diagrammatic approach the full propagator sums up all diagrams with states given at the beginning and end of the forward and backward Keldysh propagators. The non-equilibrium time evolution of the reduced density matrix elements, Eq. 3.12 can be written in the compact form

$$
P_{\chi_{2}}^{\chi_{1}}(t)=\sum_{\chi_{1}^{\prime}, \chi_{2}^{\prime}} \Pi_{\chi_{2}, \chi_{2}^{\prime}}^{\chi_{1}, \chi_{1}^{\prime}}\left(t, t_{0}\right) P_{\chi_{2}^{\prime}}^{\chi_{1}^{\prime}}\left(t_{0}\right) .
$$

This equation determines the matrix elements of the reduced density matrix at time $t$. Expanding now the time-ordered exponential in Eq. (3.13) in the tunneling Hamiltonian in terms of a power series yields

$$
\begin{aligned}
T_{K} \exp \left(-i \int_{K} d t^{\prime} H_{\mathrm{tunn}}\left(t^{\prime}\right)_{I}\right)= & \sum_{n=0}^{\infty} \frac{(-i)^{n}}{n !} \int_{K} d t_{1} \ldots \int_{K} d t_{n} \\
& T_{K}\left[H_{\mathrm{tunn}}\left(t_{1}\right)_{I} \ldots H_{\mathrm{tunn}}\left(t_{n}\right)_{I}\right] .
\end{aligned}
$$

Here, Wicks' theorem can be applied, since the tunneling Hamiltonians are bilinear in the creation and annihilation operators of the lead electrons. Performing the pairwise contractions of the lead operators yields

$$
\begin{aligned}
& \left\langle c_{\eta k \sigma}^{\dagger}(t) c_{\eta^{\prime} k^{\prime} \sigma^{\prime}}\left(t^{\prime}\right)\right\rangle=\delta_{\eta \eta^{\prime}} \delta_{k k^{\prime}} \delta_{\sigma \sigma^{\prime}} e^{\frac{i}{\hbar} \epsilon_{\eta k \sigma}\left(t-t^{\prime}\right)} f_{\eta}^{+}\left(\epsilon_{\eta k \sigma}\right) \\
& \left\langle c_{\eta k \sigma}\left(t^{\prime}\right) c_{\eta^{\prime} k^{\prime} \sigma^{\prime}}^{\dagger}(t)\right\rangle=\delta_{\eta \eta^{\prime}} \delta_{k k^{\prime}} \delta_{\sigma \sigma^{\prime}} e^{\frac{i}{\hbar} \epsilon_{\eta k \sigma}\left(t-t^{\prime}\right)} f_{\eta}^{-}\left(\epsilon_{\eta k \sigma}\right)
\end{aligned}
$$


with the Fermi function in lead $\eta$ defined as $f_{\eta}^{+}(\epsilon)=1 /\left[\exp \left(\frac{\epsilon-\mu_{\eta}}{k_{B} T}\right)+1\right]$ and $f_{\eta}^{-}(\epsilon)=1-f_{\eta}^{+}(\epsilon)$. These pairwise contractions are represented by tunneling vertices (black dots) along the Keldysh contour connected by tunneling lines. The vertices are placed at every position where a tunneling Hamiltonian arises from the expansion. A tunnel event, where an electron hops between dot and normal-conducting lead, is represented by one tunneling vertex on the Keldysh contour. The tunneling lines point towards incoming vertices, i.e. where an electron is created on the quantum dot and away from outgoing vertices. In particular, each incoming vertex gets a term $t_{\eta} d_{\sigma}^{\dagger} c_{\eta k \sigma}$ and every outgoing vertex $t_{\eta} c_{\eta k \sigma}^{\dagger} d_{\sigma}$.

The diagram Fig. 3.3 (a), which represents the time evolution of the reduced density matrix, is broken up in two types of blocks on the Keldysh contour, irreducible self energy insertions $W_{\chi_{2} \chi_{2}^{\prime}}^{\chi_{1} \chi_{1}^{\prime}}\left(t, t^{\prime}\right)$ and free propagation $\Pi_{0}^{\chi_{1} \chi_{1}^{\prime}}\left(t, t_{2}^{\prime}\right)$. The free propagation are the blocks which contain only forward and backward propagation on the Keldysh contour. The irreducible self energy insertions $W_{\chi_{2} \chi_{2}^{\prime}}^{\chi_{1} \chi_{1}^{\prime}}\left(t, t^{\prime}\right)$ symbolize transitions between different matrix elements, namely $P_{\chi_{2}^{\prime}}^{\chi_{1}^{\prime}}$ at time $t^{\prime}$ and state $P_{\chi_{2}}^{\chi_{1}}$ at time $t$.

These blocks arise due to the expansion in the tunneling Hamiltonian $H_{\text {tunn }}$. Irreducible means that any vertical cut would intersect a tunneling line. The kernel element $W_{\chi_{2} \chi_{2}^{\prime}}^{\chi_{1} \chi_{1}^{\prime}}\left(t, t^{\prime}\right)$ is defined as the sum of all irreducible diagrams and can be obtained diagrammatically, based on a perturbation expansion in the tunnel-coupling to the normal-conducting lead as displayed in Fig 3.3 (b). The number of tunneling lines in a diagram gives the order in the tunnel coupling strength.

All diagrams, irreducible self energy insertion and free propagation, can be summed up to yield the full propagator. The full propagator can be 
written in terms of a Dyson equation,

$$
\begin{aligned}
\Pi_{\chi_{2} \chi_{2}^{\prime}}^{\chi 1 \chi_{1}^{\prime}}\left(t, t^{\prime}\right)= & \Pi_{0} \begin{array}{l}
\chi 1 \chi_{1}^{\prime} \\
\chi_{2}^{\prime} \chi_{2}^{\prime}
\end{array}\left(t, t^{\prime}\right) \\
& +\sum_{\chi_{1}^{\prime \prime} \chi_{2}^{\prime \prime}} \int_{t^{\prime}}^{t} d t_{2} \int_{t^{\prime}}^{t_{2}} d t_{1} \Pi_{0} \chi_{\chi_{2} \chi_{2}}^{\chi_{1} \chi_{1}}\left(t, t_{2}\right) W_{\chi_{2} \chi_{2}^{\prime \prime}}^{\chi_{1} \chi_{1}^{\prime \prime}}\left(t_{2}, t_{1}\right) \Pi_{\chi_{2}^{\prime \prime} \chi_{2}^{\prime}}^{\chi_{1 \prime \prime}^{\prime} \chi_{1}^{\prime}}\left(t_{1}, t^{\prime}\right)
\end{aligned}
$$

with the free propagator defined as

$$
\Pi_{0}^{\chi 1 \chi_{2} \chi_{2}^{\prime}}\left(t, t^{\prime}\right)=e^{-i\left(\epsilon_{\chi_{1}}-\epsilon_{\chi_{2}}\right)\left(t-t^{\prime}\right)} \delta_{\chi_{1}, \chi_{1}^{\prime}} \delta_{\chi_{2}, \chi_{2}^{\prime}}
$$

with the energies $\epsilon_{\chi_{1}}, \epsilon_{\chi_{2}}$ of the eigenstates. Inserting the Dyson equation of the full propagator Eq. (3.19) into the equation for the non-equilibrium time evolution of the reduced density matrix elements Eq. (3.15) and taking the derivative with respect to time $t$ finally yields an exact master equation which is valid for arbitrary initial states,

$$
\dot{P}_{\chi_{2}}^{\chi_{1}}(t)=-i\left(\epsilon_{\chi_{1}}-\epsilon_{\chi_{2}}\right) P_{\chi_{2}}^{\chi_{1}}(t)+\int_{t_{0}}^{t} d t^{\prime} \sum_{\chi_{1}^{\prime} \chi_{2}^{\prime}} W_{\chi_{2} \chi_{2}^{\prime}}^{\chi_{1} \chi_{1}^{\prime}}\left(t, t^{\prime}\right) P_{\chi_{2}^{\prime}}^{\chi_{1}^{\prime}}\left(t^{\prime}\right)
$$

This is a so-called generalized master equation due to the fact that Eq. (3.21) also allows the calculation of off-diagonal density matrix elements. The first term on the right hand side describes the coherent evolution of the reduced system and the second term the dissipative coupling to the electrodes. The generalized master equation derived above is non-Markovian, i.e. the time evolution of the density matrix elements depends on previous times $t^{\prime}$. In the stationary limit, i.e. there is no explicit time dependence in the system, the kernel elements depend on the time difference $t-t^{\prime}$ only and all matrix elements of the reduced density matrix $\boldsymbol{P}$ are time independent. With the additional definition of the kernel elements $W_{\chi_{2} \chi_{2}^{\prime}}^{\chi_{1} \chi_{1}^{\prime}}=\int_{t_{0}}^{t^{\prime}} d t^{\prime} W_{\chi_{2} \chi_{2}^{\prime}}^{\chi_{1} \chi_{1}^{\prime}}\left(t, t^{\prime}\right)$ the generalized master equation in the stationary state reads

$$
0=\dot{P}_{\chi_{2}}^{\chi_{1}}=-i\left(\epsilon_{\chi_{1}}-\epsilon_{\chi_{2}}\right) P_{\chi_{2}}^{\chi_{1}}+\sum_{\chi_{1}^{\prime} \chi_{2}^{\prime}} W_{\chi_{2} \chi_{2}^{\prime}}^{\chi_{1} \chi_{1}^{\prime}} P_{\chi_{2}^{\prime}}^{\chi_{1}^{\prime}}
$$


The kernel elements $W_{\chi_{2} \chi_{2}^{\prime}}^{\chi_{1} \chi_{1}^{\prime}}$ can be obtained using diagrammatic rules. Diagrammatic rules are given at the end of Sec. 3.4 and in Appendix B we give some example calculations for diagrams.

\subsubsection{Master equation in frequency space}

In the next sections we formulate a theory to obtain current and finitefrequency noise based on the real-time diagrammatic approach. Therefore it is convenient to formulate the Master equation as introduced in Eq. (3.22) in frequency space.

The transition into frequency space of the full propagator is performed by $\Pi(\omega)=\int_{t_{0}}^{\infty} d t \Pi\left(t-t_{0}\right) e^{-i\left(\omega-i 0^{+}\right)\left(t-t_{0}\right)}$ and yields the following Dyson equation,

$$
\begin{aligned}
\boldsymbol{\Pi}(\omega) & =\boldsymbol{\Pi}_{0}(\omega)+\boldsymbol{\Pi}_{0}(\omega) \boldsymbol{W}(\omega) \boldsymbol{\Pi}(\omega) \\
& =\left[\boldsymbol{\Pi}_{0}(\omega)^{-1}-\boldsymbol{W}(\omega)\right]^{-1},
\end{aligned}
$$

with the frequency-dependent free propagator on the Keldysh contour $\boldsymbol{\Pi}_{0}(\omega)$ and the kernel $\boldsymbol{W}(\omega)$ representing the self energy of the Dyson equation due to coupling to the normal-conducting reservoir. The matrix elements of the free propagator are given by

$$
\Pi_{0}(\omega)_{\chi_{2} \chi_{2}^{\prime}}^{\chi_{1} \chi_{1}^{\prime}}=\frac{i \delta_{\chi_{1} \chi_{1}^{\prime}} \delta_{\chi_{2} \chi_{2}^{\prime}}}{\epsilon_{\chi_{2}}-\epsilon_{\chi_{1}}-\omega+i 0^{+}}
$$

where $\chi_{i}, \chi_{i}^{\prime}$ denote the different dot states at different times $t, t^{\prime}$. The kernel $\boldsymbol{W}$ is defined as the sum of all irreducible diagrams and can be obtained diagrammatically, based on a perturbation expansion in the tunnel-coupling to the normal-conducting lead (see also Fig. 3.3 (b)) and discussed in the previous subsection. The transformation into frequency space enters the diagrammatic representation by an additional horizontal bosonic line transporting the energy $\omega$.

From the frequency-dependent Dyson equation a generalized master equation can be obtained analogously to Eq. (3.22). Simply rewrite Eq. (3.23) as $\left[\boldsymbol{\Pi}_{0}^{-1}(\omega)-\boldsymbol{W}(\omega)\right] \boldsymbol{\Pi}(\omega)=\mathbf{1}$, multiply both sides with frequency $\omega$ and make 
use of the final value theorem, $\lim _{\omega \rightarrow 0}\left(i \omega+0^{+}\right) \boldsymbol{\Pi}(\omega)=\lim _{t \rightarrow \infty} \boldsymbol{\Pi}(t)=\boldsymbol{P}_{\text {stat }}$. Finally, in the stationary limit, the reduced density matrix, $\boldsymbol{P}_{\text {stat }}$, is found from the solution of a generalized master equation

$$
0=\left[\boldsymbol{\Pi}_{0}^{-1}(\omega=0)-\boldsymbol{W}(\omega=0)\right] \boldsymbol{P}_{\text {stat }}
$$

containing the coherent evolution of the reduced system described by the zero-frequency contribution to the free propagator and the dissipative coupling to the normal-conducting lead described by the zero-frequency contribution to the kernel. With the help of the solution for $\boldsymbol{P}_{\text {stat }}$, we will in the following be able to determine the expectation values of the current and the current-current correlator yielding the finite-frequency noise.

In the subsequent chapters when we present our results, we will restrict ourselves to the weak-coupling regime, performing a perturbation expansion with respect to the tunnel coupling to the normal lead. In particular, we consider diagrams with one tunneling line only. The explicit expression for the kernel can be obtained by using diagrammatic rules summarized at the end of Sec. 3.4. In the following sections we are going to present how to determine the current and the finite-frequency noise and the finite-time full counting statistics using the real-time diagrammatic approach.

\subsection{Current}

The particle current through the hybridised quantum dot is given by the operator representing the rate of change of the number of electrons in the normal lead: $\hat{I}=\frac{i}{\hbar}[\hat{N}, H]$, where $\hat{N}=\sum_{k, \sigma} c_{\eta k \sigma}^{\dagger} c_{\eta k \sigma}$. When calculating the time-dependent expectation value of the current operator, the latter acts as an external vertex on the Keldysh contour.

In order to calculate the expectation value of the charge current, $I$, the current operator is placed at the rightmost point of the Keldysh contour, see Fig. 3.4 (a), and contracted to an internal tunnel vertex via a tunnelling line. The current can be determined in an analogous way to the generalized 
transition rates presented in Sec. 3.2.2. The derivation of the equation for the current is very similar to that for the projection operator. Instead of the projection operator the current operator is inserted in Eq. (3.12). It turns out that the current can be expressed as

$$
I=\frac{1}{2} \operatorname{Tr}\left[\boldsymbol{W}_{I} \boldsymbol{P}_{\text {stat }}\right] .
$$

The kernel $\boldsymbol{W}_{I}$ can be obtained from $\boldsymbol{W}$ by replacing one of the internal tunneling vertices (black dot) by an external current vertex (open circle) 34,144. The current kernel $\boldsymbol{W}_{I}$ takes into account whether an electron enters or leaves the dot through the normal lead. The diagrammatic rules to compute the kernel $\boldsymbol{W}_{I}$ are summarised in Sec. 3.4 in lowest order in the tunnel coupling. The particle current through a quantum dot coupled

(a)

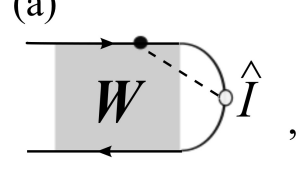

(b)

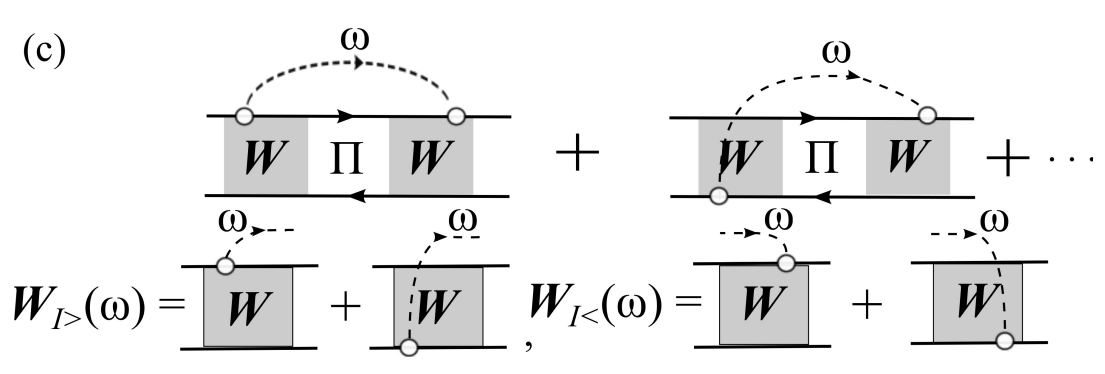

Figure 3.4: Diagrammatic representation of (a) the current, (b) the contribution to noise with both current operators in one block and (c) in different ones separated by the full propagator.

to normal-conducting leads is given by the change of particles in each lead. We will define the operator for the current through the dot symmetrized as $\hat{I}=\left(\hat{I}_{\mathrm{L}}-\hat{I}_{\mathrm{R}}\right) / 2$. The symmetrized current can be calculated using Eq. (3.26) in the same way as the current through the hybridized quantum dot. The fact that the current is symmetrized will be included into the diagrammatic 
rules to calculate the kernel $\boldsymbol{W}_{I}$. Note, that for the current itself it is not important that the current is symmetrized as due to current conservation we have $I_{\mathrm{L}}=-I_{\mathrm{R}} \equiv I$. Experimentally, the current is measured in one of the leads. At finite-frequencies the charge across the device will accumulate in time and the measured current will not be equal to the particle current. Hence, displacement currents have to be considered. Following Ref. 32], displacement currents can be taken into account following the Ramo-Shockley theorem, $\hat{I}_{\text {tot }}=\left(C_{\mathrm{R}} \hat{I}_{\mathrm{L}}-C_{\mathrm{L}} \hat{I}_{\mathrm{R}}\right) /\left(C_{\mathrm{L}}+C_{\mathrm{R}}\right)$ with $C_{\mathrm{L}}, C_{\mathrm{R}}$ the barrier capacitances. Assuming equal interface capacity between left and right reservoir the total current is equal to the symmetrized particle current. Nevertheless equal interface capacitances may still allow for an asymmetric tunnel coupling. Displacement currents are not important for the DC current, but become crucial for time-dependent and noise measurements.

Analogously, the displacement current for the quantum dot coupled to normal and superconducting lead can be considered. We consider a simple capacitive model and denote with $C_{\mathrm{N}}$ and $C_{\mathrm{S}}$ the capacitances of the tunnel barriers with the normal and superconducting lead, respectively. The number of electrons in the dot is $\hat{n}=\sum_{\sigma} d_{\sigma}^{\dagger} d_{\sigma}$. Within this model the displacement current in the normal lead is 32

$$
\hat{I}_{\mathrm{N}, \mathrm{displ}}=-\frac{C_{\mathrm{N}}}{C_{\mathrm{N}}+C_{\mathrm{S}}} \dot{\hat{n}}
$$

The total current is simply the sum of the tunnelling current and the displacement current and reads

$$
\hat{I}_{\mathrm{N}, \text { tot }}=\hat{I}_{\mathrm{N}, \text { tunn }}-\frac{C_{\mathrm{N}}}{C_{\mathrm{N}}+C_{\mathrm{S}}} \dot{\hat{n}}
$$

with the tunneling current given by

$$
\begin{aligned}
\hat{I}_{\mathrm{N}, \text { tunn }} & =-\dot{\hat{N}}=-\frac{1}{i \hbar}[\hat{N}, H] \\
& =\frac{i}{\hbar} \sum_{k, \sigma}\left(t_{\mathrm{N}} c_{\mathrm{Nk} \sigma}^{\dagger} d_{\sigma}-t_{\mathrm{N}}^{*} d_{\sigma}^{\dagger} c_{\mathrm{Nk} \sigma}\right) .
\end{aligned}
$$


Note, that the tunneling current $\hat{I}_{\mathrm{N}, \text { tunn }}$ is equal to the current $\hat{I}$ introduced in Sec. 4.3. The current $I_{\mathrm{N}}$ is positive when flowing out of the normal lead. At this stage, it is worth mentioning that if $C_{\mathrm{S}} \gg C_{\mathrm{N}}$, the displacement current in the normal lead can be neglected. This assumption is consistent with $\Gamma_{\mathrm{S}} \gg \Gamma_{\mathrm{N}}$, the limit we consider throughout this thesis, because the capacitance is proportional to the inverse of the barrier thickness.

Now, we proceed to evaluate $\dot{\hat{n}}$ :

$$
\begin{aligned}
\dot{\hat{n}} & =\frac{1}{i \hbar}[\hat{n}, H]=\hat{I}_{\mathrm{N}, \text { tunn }}+\frac{1}{i \hbar}\left[\hat{n}, H_{\text {eff }}\right] \\
& =\hat{I}_{\mathrm{N}, \text { tunn }}+\hat{I}_{\mathrm{S}, \text { tunn }},
\end{aligned}
$$

where the tunnelling current with the superconductor reads

$$
\hat{I}_{\mathrm{S}, \text { tunn }}=\frac{i}{\hbar} \Gamma_{\mathrm{S}}\left(d_{\uparrow}^{\dagger} d_{\downarrow}^{\dagger}-d_{\downarrow} d_{\uparrow}\right)
$$

Putting everything together we obtain the Ramo-Shockley theorem for the N-dot-S system:

$$
\hat{I}_{\mathrm{N}, \text { tot }}=\frac{C_{\mathrm{S}}}{C_{\mathrm{N}}+C_{\mathrm{S}}} \hat{I}_{\mathrm{N}, \text { tunn }}-\frac{C_{\mathrm{N}}}{C_{\mathrm{N}}+C_{\mathrm{S}}} \hat{I}_{\mathrm{S}, \text { tunn }}
$$

\subsection{Finite-frequency noise}

The symmetrized finite-frequency current noise is defined as the Fourier transform of $S(t)=\langle\hat{I}(t) \hat{I}(0)\rangle+\langle\hat{I}(0) \hat{I}(t)\rangle-2\langle\hat{I}\rangle^{2}$, namely of the currentcurrent correlator at different times,

$$
\begin{aligned}
S(\omega)= & \int_{-\infty}^{0} d t[\langle\hat{I}(t) \hat{I}(0)\rangle+\langle\hat{I}(0) \hat{I}(t)\rangle]\left(e^{-i \omega t}+e^{+i \omega t}\right) \\
& -4 \pi \delta(\omega)\langle\hat{I}\rangle^{2}
\end{aligned}
$$

The current in Eq. (3.33) is the tunneling current introduced in Eq. (3.26) which turns out to be equal the total current in the limit we consider throughout this thesis as discussed in the previous section. 
By construction, the finite-frequency current noise Eq. (3.33), also referred to as the power spectral density, is symmetric with respect to frequency, $S(\omega)=S(-\omega)$. It represents a real quantity, which can be measured by a classical detector 31, 147]. A classical detector is not able to distinguish between positive and negative frequencies [148] and is hence suitable to measure the symmetrized noise spectrum.

In order to calculate the current correlator, two current operators at different times have to be placed on the Keldysh contour. Diagrammatically, this means that two internal tunnelling vertices have to be replaced by external current vertices. The contributions to the current-current correlator can be grouped into two different classes, as shown in Fig. 3.4 (b) and (c). Either both current vertices are placed in the same irreducible block or in two different ones separated by a propagator.

These external operators are connected by additional bosonic (dashed) lines, carrying the frequency $\omega$ of the Fourier transform. The symmetrized finite-frequency noise can be written as 34

$$
\begin{aligned}
S(\omega)= & \frac{1}{2} \operatorname{Tr}\left[\boldsymbol{W}_{I I}(\omega) \boldsymbol{P}_{\text {stat }}+\boldsymbol{W}_{I<}(\omega) \boldsymbol{\Pi}(\omega) \boldsymbol{W}_{I>}(\omega) \boldsymbol{P}_{\text {stat }}\right] \\
& -2 \pi \delta(\omega)\langle\hat{I}\rangle^{2}+(\omega \rightarrow-\omega) .
\end{aligned}
$$

Here, the kernels $\boldsymbol{W}_{I>}(\omega)$ and $\boldsymbol{W}_{I<}(\omega)$ are the sum of all diagrams, where one tunnel vertex (black dot) is replaced by a current vertex (open circle) and a frequency line $\omega$ is attached to the current vertex. The indices $>$ and $<$ indicate whether the frequency line leaves the diagram to the right or enters it from the left as shown in Fig 3.4 (c). The kernel $\boldsymbol{W}_{I I}(\omega)$ contains diagrams with both current vertices in the same irreducible block (see Fig. 3.4 (b)).

In this last part of the section we summarize the rules to determine diagrammatically the different contributions to the kernel and current kernel as given in Refs. 64, 65 and 34. We adapt the rules to the system under investigation, which has only one normal lead. The rules for the kernel $\boldsymbol{W}(\omega)$ are:

1. Draw all topologically different diagrams with $n$ directed tunnelling 
lines connecting pairs of vertices containing lead electron operators. Assign spin index $\sigma$ and energy $\varpi$ to every tunnelling line. Additionally, assign state index $\chi$ and the corresponding energy $E_{\chi}$ to each element of the Keldysh contour connecting two vertices. Also, add an external horizontal bosonic energy line transporting the energy $\omega$ to each diagram, which results from the Fourier transform.

2. For each time segment between two adjacent vertices write a resolvent $1 /\left(\Delta E(t)+i 0^{+}\right)$with $\Delta E$ being the difference between all backwardgoing minus forward-going energies, including tunnelling lines transporting the energy $z$ as well as the external line transporting the energy $\omega$.

3. Each vertex containing a dot operator $d_{\sigma}^{(\dagger)}$ gives rise to a matrix element $\left\langle\chi^{\prime}\left|d_{\sigma}^{(\dagger)}\right| \chi\right\rangle$ where $\chi\left(\chi^{\prime}\right)$ is the dot state entering (leaving) the vertex with respect to the Keldysh contour. Consequently, for each vertex connecting a doubly-occupied state $d$ to the up state $\uparrow$, the diagram acquires a factor $(-1)$.

4. Each tunnelling line contributes with a factor $\frac{1}{2 \pi} \Gamma_{N} f_{N}(\varpi)$ for a backwardgoing line with respect to the closed time path and a factor $\frac{1}{2 \pi} \Gamma_{\mathrm{N}}\left[1-f_{\mathrm{N}}(\varpi)\right]$ for a forward-going contribution.

5. Each diagram has an overall prefactor $(-i)(-1)^{b+c}$, where $b$ is the total number of vertices on the backward propagator and $c$ is the number of crossings of tunnelling lines.

6. Finally, sum over the spin $\sigma$ and integrate over the energies $\varpi$ of tunnelling lines and sum over all diagrams that contribute to the same kernel element.

As a next step we provide the additional rules to determine the blocks containing one or two current operators $\boldsymbol{W}_{I}(\omega)$ and $\boldsymbol{W}_{I I}(\omega)$. 
7. Replace one (two) tunnel vertex by a current vertex to calculate diagrams contributing to the kernels $\boldsymbol{W}_{I}(\omega)\left(\boldsymbol{W}_{I I}(\omega)\right)$. Note, that the current vertex (open circle) might also be placed on the start or end point of the diagram.

8. Multiply each diagram by a prefactor, determining the position of the current vertex inside the diagram: we have to multiply each diagram by a factor of $(-1)$ for a current vertex on the upper (lower) Keldysh time branch and a particle tunnelling into (out of) the normal lead. In the two other cases multiply the diagram with a factor of $(+1)$.

9. The diagrams contributing to $\boldsymbol{W}_{I>}(\omega), \boldsymbol{W}_{I<}(\omega)$ have open external frequency lines to the right or left side attached to the current vertex. Diagrams with frequency lines leaving the diagram to the right contribute to the kernel $\boldsymbol{W}_{I>}(\omega)$, while diagrams with with frequency lines coming from the left contribute to $\boldsymbol{W}_{I<}(\omega)$.

The diagrammatic rules introduced above are for the system with only one normal-conducting lead. When considering a quantum dot with two normal leads then we have to multiply each diagram by a factor of $+1 / 2$ for a current vertex on the upper (lower) Keldysh time branch, which describes a particle tunneling into the right (left) lead or out of the left (right) lead. In the four other cases multiply the diagram with a factor of $-1 / 2$. The factor $1 / 2$ takes into account that the total current is symmetrized.

In Appendix B we give examples for the different types of diagrams using the diagrammatic rules introduced here. Additionally, in Appendix C we present the kernels contributing to the finite-frequency noise in the unidirectional transport regime for the dot coupled to a normal and a superconducting lead. 


\subsection{Finite-time full counting statistics}

In this section we introduce a theory to calculate the finite-time full counting statistics for transport through a strongly interacting quantum dot and also the current and the finite-frequency noise.

We are interested in the transport properties of a nanoscale conductor such as a quantum dot coupled to leads. The system is completely captured by the time evolution of the reduced density matrix, which we obtain by tracing out the electronic leads. As introduced in the previous Sec. 3.2 the time evolution of the reduced density matrix $\boldsymbol{P}(t)$ obeys a generalized master equation, e.g Eq. (3.21). Here, we aim to formulate a theory which allows us to study the statistics of the transferred charges. It is therefore advantageous to resolve the reduced density matrix $\boldsymbol{P}(t) \rightarrow \boldsymbol{P}(N, t)$ in components corresponding to the number of electrons $N$ that have tunnelled through the system in the time interval from 0 to $t$. Summing over all values of $N$ yields the known reduced density matrix, $\boldsymbol{P}(t)=\sum_{N} \boldsymbol{P}(N, t)$.

The evolution of the reduced density matrix for transport in a nanoscale conductor is described by a generalized non-Markovian (NM) master equation (NMME) of the form 60, 149, 150

$$
\frac{d}{d t} \boldsymbol{P}(N, t)=\sum_{N^{\prime}} \int_{0}^{t} d t^{\prime} \boldsymbol{W}\left(N-N^{\prime}, t-t^{\prime}\right) \boldsymbol{P}\left(N^{\prime}, t^{\prime}\right)+\gamma(N, t)
$$

This NMME allows us in the following to study the statistics of the number of transferred charges $N$ through a system in a time interval $t$. We consider the time evolution of the reduced system from time $t=0$ where the counting begins. The memory kernel $\boldsymbol{W}$ governs the influence of the reservoir on the dynamics of the system. It depends only on the time difference $t-t^{\prime}$ because we assume that the system is not explicitly driven by any time-varying field. The second term of the NMME Eq. (3.35) is the inhomogeneous term $\gamma(N, t)$, which captures the initial correlation between system and reservoir. This term describes the system's memory of its history prior to some time $t=0$. In the long time limit $t \rightarrow \infty$ the initial correlations between system and 
reservoir decay, i.e. $\gamma(N, t) \rightarrow 0$ and can be neglected in the master equation Eq. (3.35).

However, for finite times it is crucial to include the inhomogeneous term 40 , 60,63. This allows to fully include NM dynamics. Note, the theory to obtain the finite-frequency noise introduced in Sec. 3.4 captures also NM effects.

The full counting statistics (FCS) of electron transport in nanoscale conductors reveal fundamental information about the current fluctuations in the system. The FCS gives complete information of the statistics of the number of charges $N$ transferred through the system in a given time $t$, defined as the probability distribution $p(N, t)[26,27]$. The probability distribution $p(N, t)$ can be expressed in terms of the moment generating function (MGF) defined as

$$
G(\chi, t)=\sum_{N} p(N, t) e^{i N \chi}
$$

where $\chi$ is the so-called counting field. The counting field $\chi$ is introduced via a Fourier transformation, $f(\chi, t)=\sum_{N=-\infty}^{\infty} f(N, t) e^{i N \chi}$ and is the conjugated variable to the number of charges $N$. The derivatives with respect to the counting field $\chi$ give the moments of $k$ th order

$$
<n^{k}(t)>=\left.\frac{\partial G(\chi, t)}{\partial(i \chi)^{k}}\right|_{\chi \rightarrow 0} .
$$

Alternatively, the FCS can be expressed in terms of the cumulant generating function $(\mathrm{CGF})$ which is defined as

$$
S(\chi, t)=\ln \sum_{N} p(N, t) e^{i N \chi}=\ln G(\chi, t)
$$

and the current cumulants are obtained by performing the derivatives with respect to the counting field $\chi$ and sending $\chi \rightarrow 0$. Furthermore the probability distribution $p(N, t)$ can be obtained from the reduced density matrix by taking the trace over the system degrees of freedom

$$
p(N, t)=\operatorname{Tr}[\boldsymbol{P}(N, t)] .
$$


The MGF can be expressed in terms of the reduced density matrix as

$$
G(\chi, t)=e^{S(\chi, t)}=\operatorname{Tr}[\boldsymbol{P}(\chi, t)]
$$

with the $\chi$-dependent density matrix $\boldsymbol{P}(\chi, t)=\sum_{N} \boldsymbol{P}(N, t) e^{i N \chi}$.

The aim is to study the FCS described by the NMME Eq. 3.35 within the framework of the diagrammatic real-time approach [64,65]. Introducing the Laplace transform, $f(\chi, z)=\sum_{N} \int_{0}^{\infty} d t f(N, t) e^{i N \chi-z t}$, NMME Eq. 3.35 reads,

$$
z \boldsymbol{P}(\chi, z)-\boldsymbol{P}(\chi, t=0)=\boldsymbol{W}(\chi, z) \boldsymbol{P}(\chi, z)+\gamma(\chi, z)
$$

Rewriting the equation we get

$$
\boldsymbol{P}(\chi, z)=\boldsymbol{\Pi}(\chi, z)[\boldsymbol{P}(\chi, t=0)+\gamma(\chi, z)]
$$

with $\Pi(\chi, z)=[z-\boldsymbol{W}(\chi, z)]^{-1}$.

To understand the time evolution of the reduced density matrix in $\chi$-space Eq 3.42 we divide the system into two time intervals, a non-counting interval $(\chi=0)$ prior to some time $t=0$ where the counting begins and an interval where the counting is active. A schematic sketch of the time evolution of the system is shown in Fig. 3.5. At the initial state at $t_{0}$ system and reservoir are separable. The system envolves without counting from $t_{0}$ to $t=0$ where the counting begins. At $t=0$ system and reservoir are no longer separable. In the interval from $t=0$ to the final time $t$ counting takes place.

We assume now that the system evolves from the initial state at $t_{0}=-\infty$ such that the occupation probabilities at $t=0$, where the counting begins have reached the stationary state. Consequently the reduced density matrix has reached its stationary state at $t=0$, namely $\boldsymbol{P}(N, t=0)=\delta_{N, t=0} \boldsymbol{P}_{\text {stat }}$. Taking this assumption into consideration we can write the reduced density matrix Eq. (3.42) as

$$
\boldsymbol{P}(\chi, z)=\boldsymbol{\Pi}(\chi, z)[1+\boldsymbol{\Gamma}(\chi, z)] \boldsymbol{P}_{\text {stat }}
$$


where the stationary density matrix can be obtained by solving $\boldsymbol{W}(\chi=0, z=$ $0) \boldsymbol{P}_{\text {stat }}=0$. The resulting equation for the reduced density matrix Eq. 3.43

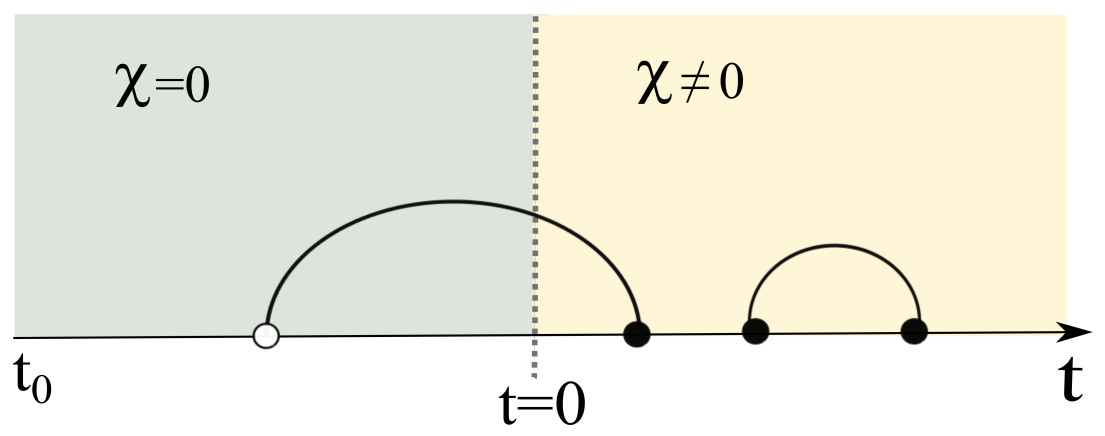

Figure 3.5: Schematic picture of the system. The reduced density matrix evolves forward in time from the initial time $t_{0}$ to $t=0$ where the counting begins. Solid circles denote tunnel vertices with a finite counting contribution and open circles denote vertices in the non-counting regime.

contains two different types of self-energy kernels, $\boldsymbol{W}(\chi, z)$ and $\boldsymbol{\Gamma}(\chi, z)$. The kernel $\boldsymbol{\Gamma}(\chi, z)$ represents the inhomogeneity term and contains all diagrams connecting the counting and non-counting region. We are going to introduce the inhomogeneity term $\boldsymbol{\Gamma}(\chi, z)$ in detail in the following. We assume the coupling to the reservoir is weak $\left(\Gamma_{\eta} \ll k_{B} T\right.$, where $k_{B}$ is the Boltzmann constant and $T$ is the absolute temperature; $\eta$ is the lead index). Considering this condition we make use of the diagrammatic real-time approach in the pertubative regime for the tunnel coupling to the lead [64, 65] using the Keldysh formalism as introduced in Sec. 3.2. Hence the kernels, $\boldsymbol{W}(\chi, z)$ and $\boldsymbol{\Gamma}(\chi, z)$, can be determined by using diagrammatic rules, see Sec. 3.4 . The only extension to the diagrammatic appraoch we have to make here is to incorporate the counting field into the approach. Assume that the counting in lead $\eta$ is represented by a counting field $\chi_{\eta}$. The counting in lead $\eta$ can be included by adding the counting field $\chi_{\eta}$ to the tunneling Hamiltonian $H_{\text {tunn }}$, where each tunnel event is represented by a vertex on the Keldysh contour. In practice, the counting field $\chi_{\eta}$ can be included through the replacement, $t_{\eta} \rightarrow t_{\eta} e^{i \chi_{\eta} / 2}$ for a tunneling vertex on the upper Keldysh contour and $t_{\eta} \rightarrow$ 
$t_{\eta} e^{-i \chi_{\eta} / 2}$ on the lower contour, respectively. Here, we have to distinguish between two types of tunnel vertices, namely between counting and noncounting vertices. For all vertices describing tunneling processes within the counting interval, represented by black dots on the Keldysh contour, the counting field has to be incorporated by the replacement introduced above. While all vertices in the non-counting interval, depicted as open circles on the Keldysh contour, are the same sort of tunnel vertices as introduced in Sec. 3.2.

The kernel $\boldsymbol{W}(\chi, z)$ comprises all tunnel processes taking place in the counting interval, i.e all tunnel vertices are counting-vertices (black dots) on the Keldysh contour. This kernel is of the same type as the self-energy kernel introduced in Sec. 3.2 with the extension of incorporating the counting fields into the tunneling processes. The kernel $\boldsymbol{\Gamma}(\chi, z)$ represents the inhomogeneity term and involves tunneling processes where vertices between counting and non-counting interval get contracted. In first order in the tunnel coupling, i.e. for all diagrams with only one tunneling line, we find for the inhomogeneity term

$$
\boldsymbol{\Gamma}(\chi, z)=\frac{1}{z}[\tilde{\boldsymbol{W}}(\chi, z=0)-\tilde{\boldsymbol{W}}(\chi, z)]
$$

using the assumption that the system evolves from $t_{0}=-\infty$ such that the reduced density matrix $\mathbf{P}(N, t)$ has found its stationary state at $t=0$ when the counting begins. The matrix elements of the kernel $\tilde{\boldsymbol{W}}(\chi, z)$ are those diagrams describing contractions between counting and non-counting vertices. Since we only consider first order diagrams, the rightmost vertex is a tunneling vertex in the counting interval (black dot), while the leftmost is a non-counting vertex (open circle). Note, when considering also higher order tunnel processes, i.e beyond the weak coupling regime the expression Eq. (3.44) becomes more complex and additional terms appear. The explicit expression for both types of kernel $\boldsymbol{W}(\chi, z)$ and $\boldsymbol{\Gamma}(\chi, z)$ can be obtained by using the diagrammatic rules of Sec. 3.4, with the counting field included as described above. A concrete example for diagrams contributing to $\boldsymbol{\Gamma}(\chi, z)$ 
can be found in Appendix B.

In Chapter 5 we apply the theory introduced here to our model of a quantum dot strongly coupled to superconductor and weakly to a normal conducting lead. We restrict ourselves to the case when the temperature is larger than the tunnel-coupling strength of the normal-conducting lead $\left(k_{\mathrm{B}} T \ll \Gamma_{\mathrm{N}}\right)$. In this particular regime, the Kondo correlations due to the coupling with the normal lead are negligible and we can treat the tunneling with the normal lead to the lowest non-vanishing order. Next-to-leading-order corrections in the tunnel coupling $\Gamma_{\mathrm{N}}$ would mainly yield small quantitative corrections since the perturbation expansion is performed in a small parameter. For the subgap transport characteristics of the system, the superconductor can be described by means of an effective Hamiltonian as introduced in Sec. 3.1. which becomes exact in the regime of infinite superconducting gap. However, the effective Hamiltonian still describes well the subgap transport features even for finite values of the gap as long as the temperature is larger than the Kondo temperature related to the Kondo screening by the quasiparticle excitations in the superconductor. A detailed study of the reliability of the approximations can be found in Ref. 94].

This theory allows to obtain the reduced density matrix for a NMME, which gives direct access to the frequency depend MGF Eq. 3.40). The MGF allows in general to determine the current and the finite-frequency noise directly, as well as the finite-time FCS.

Thus we introduced here an alternative approach to the one presented in Sec. 3.3 and Sec. 3.4 to determine the current and the finite-frequency noise. One advantage of the MGF is that we can not only calculate the symmetrized current and noise, but also its cross- and auto-correlations separately by taking the derivatives of the MGF with respect to different counting fields of the different contacts. This enables us to determine also the noise associated to the charge accumulating on the nanostructure. Beside this, to determine the MGF one has to calculate only two types of self-energy kernels, $\boldsymbol{W}(\chi, z)$ and $\tilde{\boldsymbol{W}}(\chi, z)$. Lastly, the knowledge of the MGF also enables to calculate 
higher order current cumulants.

The non-Markovian theory for finite-time FCS that we have set up here captures also the quantum noise regime, i.e when the noise frequency is the largest energy scale, $\omega>k_{B} T, e V$, namely a noise frequency larger than temperature and applied bias voltage. This is different to other non-Markovian theories for counting statistics found in the literature as in Ref. [60]. The reason for this is the nature of the counting, i.e. whether the number of transferred electrons is considered to be a quantum or classical variable. The difference manifests in the inhomogeneity term. In the classical case the inhomogeneity term is independent of the counting field $\chi$, i.e contractions between counting and non-counting field are not taken into account. Hence, the knowledge of the kernel $\boldsymbol{W}(\chi, z)$ is sufficient [60]. In our approach we consider the number operator counting the transferred charges quantum mechanically. Consequently, contractions between counting and non-counting field arise, which leads to a new type of kernel, $\tilde{\boldsymbol{W}}(\chi, z)$ and the inhomogeneity term is not independent of the counting field. The difference does not play a role for the first cumulant, the average current, since memory effects do not play a role for the stationary limit. A detailed discussion of the classical and quantum-mechanical counting in NMME can be found in Ref. 63.

Let us now define all relevant quantities. The average current in Laplace space is given by

$$
\langle I(z)\rangle=z\langle n(z)\rangle=\left.z \frac{\partial}{\partial(i \chi)} G(\chi, z)\right|_{\chi \rightarrow 0} .
$$

The symmetrized finite-frequency noise can be expressed as in Ref. [60] by

$$
S(\omega)=-\frac{\omega^{2}}{2} \frac{\partial^{2}}{\partial(i \chi)^{2}}(G(\chi, z=i \omega)+(z=-i \omega))_{\chi \rightarrow 0} .
$$

The current Eq. (3.45) and noise formula Eq. 3.46) coincide with the current and finite-frequency noise introduced in the previous section Eq. (3.26) and Eq. (3.34) apart from a factor of two in the definition for the finite-frequency 
noise. Nevertheless, the definition of the noise in Eq. 3.46) is convenient since in the FCS the Fano factor is defined as $F=S / I$. This shows that after having calculated the MGF you have direct access to the current and finite-frequency noise by simply taking the first and second derivative of the MGF.

At this stage, we should point out that the above derivation focuses on particle currents only. As already discussed in section 3.3, displacement currents have to be considered at finite frequencies, since the current is not conserved due to charge accumulation in the system. Nevertheless, current conservation can be taken into account by the inclusion of the correct counting fields of the electronic reservoirs following the Ramo-Shockley theorem 32 . For example, in the case of a quantum dot coupled to two leads left and right the total symmetrized noise can be obtained by taking the derivative with respect to a total counting field, with $\chi_{\mathrm{L}}=-\chi_{\mathrm{R}}=\chi_{\text {tot }} / 2$ under the assumption of symmetrized barrier capacitances $\left(C_{\mathrm{L}}=C_{\mathrm{R}}\right)$.

However, the MGF allows us to calculate not only the average current, it gives also access to the full statistical description of the transferred electrons, namely the FCS.

The cumulants defining the number of transmitted charges are defined as the $k$ th derivative of the CGF

$$
\left\langle\kappa^{(k)}(t)\right\rangle=\left.\frac{\partial^{k} S(\chi, t)}{\partial(i \chi)^{k}}\right|_{\chi \rightarrow 0}
$$

and the $k$ th time averaged current cumulants are defined as

$$
\left\langle I^{k}(t)\right\rangle_{c}=\frac{d}{d t}\left\langle\kappa^{(k)}(t)\right\rangle
$$

We are interested in the Fano factor of the cumulants which is defined as

$$
F^{k}=\left\langle\kappa^{(k)}(t)\right\rangle /\left\langle\kappa^{(1)}(t)\right\rangle
$$

and the time averaged $k$ th order Fano factor as

$$
F_{c}^{k}=\left\langle I^{k}(t)\right\rangle_{c} /\left\langle I^{1}(t)\right\rangle_{c}
$$


respectively.

Since the finite-time FCS theory results in the MGF it is reasonable to express the cumulants Eq. 3.47) in terms of the moments Eq. (3.37).

All cumulants can be calculated recursively in the following way

$$
\left\langle\kappa^{(k)}(t)\right\rangle=\left\langle n^{k}(t)\right\rangle-\sum_{m=1}^{k-1}\left(\begin{array}{c}
k-1 \\
m-1
\end{array}\right)\left\langle\kappa^{(m)}(t)\right\rangle\left\langle n^{k-m}(t)\right\rangle
$$

and we find for the first three cumulants,

$$
\begin{gathered}
\left\langle\kappa^{(1)}(t)\right\rangle=\left\langle n^{1}(t)\right\rangle \\
\left\langle\kappa^{(2)}(t)\right\rangle=\left\langle n^{2}(t)\right\rangle-\left\langle\kappa^{(1)}(t)\right\rangle\left\langle n^{1}(t)\right\rangle=\left\langle n^{2}(t)\right\rangle-\left\langle n^{1}(t)\right\rangle\left\langle n^{1}(t)\right\rangle \\
\left\langle\kappa^{(3)}(t)\right\rangle=\left\langle n^{3}(t)\right\rangle-\left(\left\langle\kappa^{(1)}(t)\right\rangle\left\langle n^{2}(t)\right\rangle+2\left\langle\kappa^{(2)}(t)\right\rangle\left\langle n^{1}(t)\right\rangle\right) \\
=\left\langle n^{3}(t)\right\rangle-\left(\left\langle n^{1}(t)\right\rangle\left\langle n^{2}(t)\right\rangle+2\left(\left\langle n^{2}(t)\right\rangle-\left\langle n^{1}(t)\right\rangle\left\langle n^{1}(t)\right\rangle\right)\left\langle n^{1}(t)\right\rangle\right)
\end{gathered}
$$

and all other higher order cumulants can be obtained analogously. Note, that we used here that $G(\chi=0, t)=1$ for a normalized density matrix.

\subsection{Multi-time full counting statistics}

In this section we are going to extend the previously introduced theory to multi-times. As introduced in the previous Sec. 3.5 the probability distribution $p(N, t)$ of the transferred charges through the device in a given time gives complete information on the statistics of the number of charges which have been transported. The probability distribution can be obtained from the reduced density matrix by taking the trace over the system degrees of freedom Eq. (3.39). In order to obtain the multi-times FCS we can now introduce the so-called joint probability distribution $p\left(N_{1}, t_{1} ; N_{2}, t_{2} ; \ldots ; N_{k-1}, t_{k-1} ; N_{k}, t_{k}\right)$ which contains the correlations at different times.

The distribution $p\left(N_{1}, t_{1} ; \ldots N_{k}, t_{k}\right)$ is the probability that $N_{1}$ electrons have been transferred after time $t_{1}$ and $N_{2}$ after some time $t_{2}$ and so on until $N_{k}$ electron have been passed after time $t_{k}$. 
The multi-time FCS presented here has been introduced previously in Ref. 62, 104. This allows us to study also the frequency-dependence of higher-order current correlations as the skewness. For simplicity we consider only the unidirectional transport regime, i.e we assume that the applied bias voltage is the largest energy scale in the system and back-tunneling to the reservoir is suppressed. In this regime the dynamics of the system are governed by a Markovian master equation. Hence, we can neglect the inhomogeneity term in Eq. (3.35) and do not need to consider any initial correlations between system and reservoir.

The aim is to derive a MGF for multi-times. Namely, we are interested in the current correlations, which correlates the number of electrons that have been transferred through the device at $k$ different times. The idea is to divide the time evolution of the system into different time intervals, as shown in Fig. 3.6. Each time $t_{j}$ is related to a number of electrons $N\left(t_{j}\right)$ that have been passed across the device in that time. Since the number of transferred electrons is conjugated to a counting field, we associate a different counting field $\chi_{i}$ for each propagation. Consequently, more than one counting field can be active in one time interval. Precisely, if we consider the time interval $\Delta t_{k-j}=t_{k-j+1}-t_{k-j}$ exactly $\tilde{\chi}_{j}=\sum_{i=k+1-j}^{k} \chi_{i}$ counting fields are active.

In $\chi$ space the joint probability distribution can be expressed in terms of the MGF,

$$
\sum_{N_{1}, N_{2}, \ldots N_{k}} e^{i N_{1} \chi_{1}+i N_{2} \chi_{2}+\ldots+i N_{k} \chi_{k}} p^{(k)}[\boldsymbol{N}, \boldsymbol{t}]=G^{(k)}[\boldsymbol{\chi}, \boldsymbol{t}]
$$

with $\boldsymbol{N}=\left(N_{1}, \ldots . ., N_{k}\right)_{T}, \boldsymbol{t}=\left(t_{1}, \ldots ., t_{k}\right)_{T}$ and $\boldsymbol{\chi}=\left(\chi_{1}, \ldots ., \chi_{k}\right)_{T}$, where the superscript $k$ reminds us that we have introduced $k$ different times.

Before we introduce the multi-time MGF, we define the Markovian master equation for one time interval. For only one time interval, the Markovian master equation describing the time evolution of the reduced density matrix is given by

$$
\boldsymbol{P}(\chi, t)=\boldsymbol{\Pi}\left(\chi, t-t_{0}\right) \boldsymbol{P}\left(\chi, t_{0}\right)
$$

where $\boldsymbol{\Pi}$ is the full propagator. We assume that the counting starts at $t_{0}$, i.e 


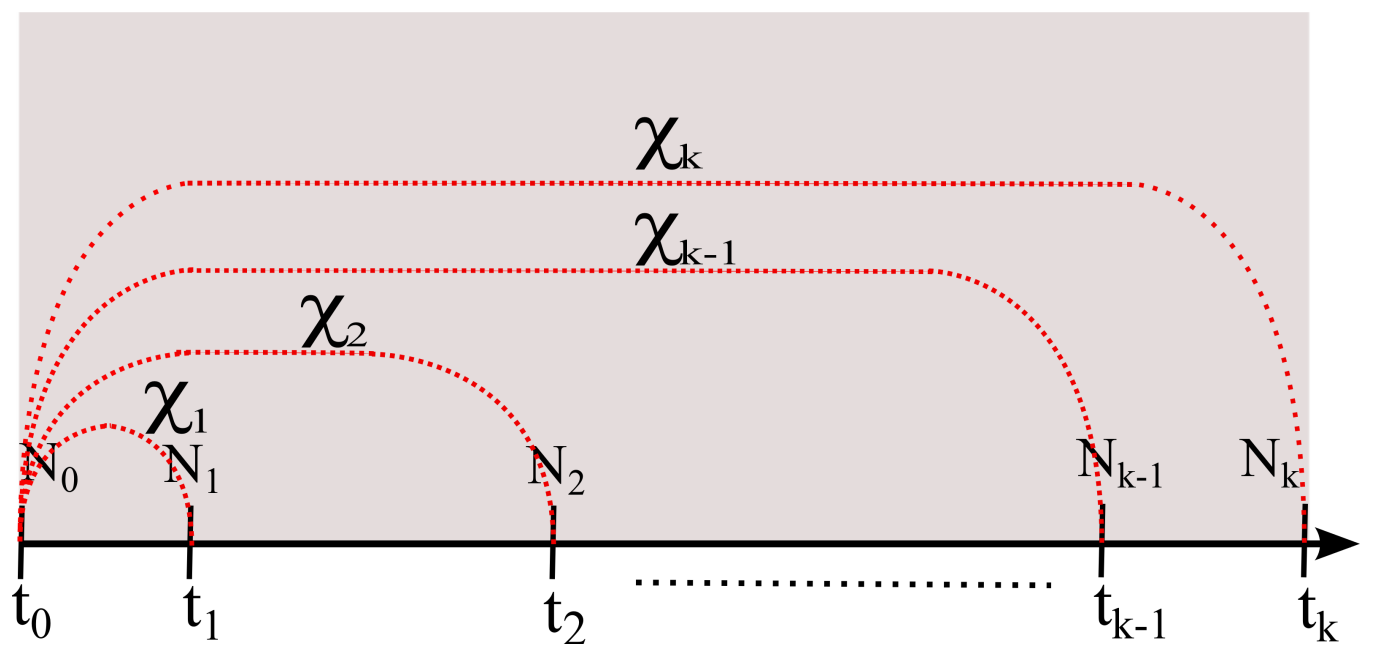

Figure 3.6: Schematic sketch of the time evolution of the system. Each time interval is related to a number of electrons which have been passed the device and a counting field respectively.

no electrons have been passed at that time and we write $\boldsymbol{P}\left(\chi, t_{0}\right)=\boldsymbol{P}\left(t_{0}\right)=$ $\boldsymbol{P}_{\text {stat }}$. In order to solve for the joint probability distribution we have to partition the reduced density matrix Eq. 3.56 and the full propagator in terms of different time intervals, since the trace over the reduced density matrix yields the MGF. The multi-time MGF is given by

$$
\begin{aligned}
G^{(k)>}[\boldsymbol{\chi}, \boldsymbol{t}] & =\operatorname{Tr}\left[\boldsymbol{\Pi}\left(\tilde{\chi}_{1}, \Delta t_{0}\right) \boldsymbol{\Pi}\left(\tilde{\chi}_{2}, \Delta t_{1}\right) \ldots \boldsymbol{\Pi}\left(\tilde{\chi}_{j}, \Delta t_{k}\right) \boldsymbol{P}_{\text {stat }}\right] \\
& =\operatorname{Tr}\left[\prod_{j=1}^{k} \boldsymbol{\Pi}\left(\tilde{\chi}_{j}, \Delta t_{k-j}\right) \boldsymbol{P}_{\text {stat }}\right]
\end{aligned}
$$

with $\tilde{\chi}_{j}=\sum_{i=k+1-j}^{k} \chi_{i}, \Delta t_{j}=t_{j+1}-t_{j}$ and $>$ denotes that we assume a specific time ordering, namely $t_{k}>t_{k-1}>\ldots . t_{1}>t_{0}$. By introducing the time ordering operator $\mathcal{T}$ we can obtain the full MGF as

$$
G^{(k)}[\boldsymbol{\chi}, \boldsymbol{t}]=\mathcal{T} \operatorname{Tr}\left[\prod_{j=1}^{k} \Pi\left(\tilde{\chi}_{j}, \Delta t_{k-j}\right) \boldsymbol{P}_{\text {stat }}\right] .
$$


We are interested in the finite-frequency cumulants. Therefore it is useful to transform Eq. 3.57 to the Laplace space, which yields

$$
G^{(k)>}[\boldsymbol{\chi}, \boldsymbol{z}]=\operatorname{Tr}\left[\prod_{j=1}^{k} \boldsymbol{\Pi}\left(\tilde{\chi}_{j}, \tilde{z}_{j}\right) \boldsymbol{P}_{\text {stat }}\right],
$$

with $\tilde{z}_{j}=\sum_{i=k+1-j}^{k} z_{i}$ and the frequency-dependent propagator $\Pi(z, \chi)=$ $[\boldsymbol{z}-\boldsymbol{W}(\chi)]^{-1}$ which can be calculated using the previously introduced diagrammatic approach. The $k$ th order moments are given by the derivative with respect to the respective counting fields as

$$
n^{(k)>}(\boldsymbol{z})=\partial_{i \chi_{1}} \partial_{i \chi_{2}} \ldots \partial_{i \chi_{k}} G^{(k)>}[\boldsymbol{\chi}, \boldsymbol{z}]
$$

In order to obtain the frequency-dependent noise and skewness as well as higher order current-cumulants, we have to define the averaged current cumulants Eq. 3.48 in frequency space. The $k$-time averaged current cumulants in frequency space are defined by the symmetrized version of the power spectrum as

$$
S^{(k)}\left(\omega_{1}, \ldots, \omega_{k}\right)=\int_{-\infty}^{\infty} d t_{1} \ldots . d t_{N} e^{-i \omega_{1} t_{1}} \ldots . . e^{-i \omega_{k} t_{k}} \mathcal{T}_{\mathcal{S}}\left\langle I\left(t_{1}\right) \ldots . I\left(t_{k}\right)\right\rangle
$$

with the symmetrization operator $\mathcal{T}_{\mathcal{S}}$, which symmetries over all possible switches of times or frequencies, respectively. For example, for $k=2$ we get $S^{(2)}\left(\omega_{1}, \omega_{2}\right)=\int_{-\infty}^{\infty} d t_{1} d t_{2} e^{-i\left(\omega_{1} t_{1}+\omega_{2} t_{2}\right)}\left[\left\langle I\left(t_{1}\right) I\left(t_{2}\right)\right\rangle+\left\langle I\left(t_{2}\right) I\left(t_{1}\right)\right\rangle\right]$, which is the finite-frequency noise as introduced in Eq. (3.33).

We can express the multi-time current cumulants $\left\langle I\left(t_{1}\right) \ldots I\left(t_{k}\right)\right\rangle_{c}$ in terms of the CGF $S^{(k)}[\boldsymbol{\chi}, \boldsymbol{t}]$. The current cumulants are defined by $\left\langle I^{k}(t)\right\rangle_{c}=$ $\left.\frac{d}{d t} \frac{\partial^{k} S(\chi, t)}{\partial(i \chi)^{k}}\right|_{\chi \rightarrow 0}$ (see Eq. (3.48)). In the following derivation we consider only the ${ }^{\prime}>$ ' part, i.e the unsymmetrized part with respect to the time ordering $t_{1}<t_{2}<. .<t_{k-1}<t_{k}$. Replacing the N-time current cumulants in terms of the CGF and using the property of a Fourier transform of a derivative $\left(\frac{d^{n} f(x)}{d x^{n}} \rightarrow(i \omega)^{n} F(\omega)\right)$, we obtain for the averaged current cumulants in 
frequency-space

$$
\begin{aligned}
S^{(k)>}\left(\omega_{1}, \ldots, \omega_{k}\right)= & \left.\int_{-\infty}^{\infty} d t_{1} \ldots d t_{N} e^{-i \omega_{1} t_{1}} \ldots e^{-i \omega_{k} t_{k}} \partial_{t_{2}} \ldots \partial_{t_{k}} \partial_{i \chi_{2}} \ldots \partial_{i \chi_{k}} S^{k>}[\boldsymbol{\chi}, \boldsymbol{t}]\right|_{\chi=\mathbf{0}} \\
= & \left(i \omega_{1}\right) \ldots\left(i \omega_{k}\right) \int_{-\infty}^{\infty} d t_{1} \ldots d t_{N} e^{-i \omega_{1} t_{1}} \ldots e^{-i \omega_{k} t_{k}} \times \\
& \left.\partial_{i \chi_{2}} \ldots \partial_{i \chi_{k}} S^{k>}[\boldsymbol{\chi}, \boldsymbol{t}]\right|_{\boldsymbol{\chi}=\mathbf{0}} .
\end{aligned}
$$

At this stage we split the integral in order to introduce the Laplace transform $\left(f(z)=\int_{0}^{\infty} d t e^{-z t} f(t)\right)$ and set $i \omega=z$, so that the we find for the polyspectrum [40]

$$
S^{(k)>}\left(z_{1}, \ldots, z_{k}\right)=\left.z_{1} \ldots z_{k} \partial_{i \chi_{2}} \ldots \partial_{i \chi_{k}} S^{(k)>}[\boldsymbol{\chi}, \boldsymbol{z}]\right|_{\boldsymbol{\chi}=\mathbf{0}}
$$

with the multi-time CGF

$$
S^{(k)>}[\boldsymbol{\chi}, \boldsymbol{z}]=\ln G^{(k)>}[\boldsymbol{\chi}, \boldsymbol{z}]=\ln \operatorname{Tr}\left[\prod_{j=1}^{k} \boldsymbol{\Pi}\left(\tilde{\chi}_{j}, \tilde{z}_{j}\right) \boldsymbol{P}_{\text {stat }}\right] .
$$

It might be advantage to express the multi-time cumulates in terms of the moments. This can be done recursively as introduced in Eq. (3.51). The difference here is that we have to account for the different times. For example for $k=2$ we find for the polyspectrum Eq. 3.63,

$$
S^{(2)>}\left(z_{1}, z_{2}\right)=z_{1} z_{2} n^{(2)}\left(z_{1}, z_{2}\right)-I\left(z_{1}\right) I\left(z_{2}\right)
$$

and the analog for $k=3$

$$
\begin{aligned}
S^{(3)>}\left(z_{1}, z_{2}, z_{3}\right) & =z_{1} z_{2} z_{3} n^{(3)}\left(z_{1}, z_{2}, z_{3}\right)-z_{2} z_{3} I\left(z_{1}\right) n^{(2)}\left(z_{2}, z_{3}\right) \\
& -z_{1} z_{3} I\left(z_{2}\right) n^{(2)}\left(z_{1}, z_{3}\right)-z_{1} z_{2} I\left(z_{3}\right) n^{(2)}\left(z_{1}, z_{2}\right) \\
& +2 I\left(z_{1}\right) I\left(z_{2}\right) I\left(z_{3}\right)
\end{aligned}
$$

with $n^{(1)(z)}=z I(z)$.

In order to symmetrize the spectrum in frequency space, also the part corresponding to all negative frequencies $z$ has to be added and the sum 
over all possible switchings of frequencies has to be performed. When the expression is symmetrized with respect to the frequencies $S^{(k)}$ and evaluated at $\boldsymbol{z}=\boldsymbol{i} \boldsymbol{\omega}$, it becomes proportional to the Dirac delta function $\delta\left(\omega_{1}+\ldots+\omega_{N}\right)$ due to frequency conservation implied by time-translational invariance. 


\section{Chapter 4}

\section{Finite-frequency noise in transport through a quantum dot}

In this chapter we present results for the finite-frequency current noise in transport through a quantum dot coupled to normal and superconducting leads in different transport regimes with respect to the applied bias voltage or the noise frequency. In the first section we discuss the current and finite-frequency noise through a single-level quantum dot coupled to normal conducting leads obtained using the diagrammatic real-time approach for noise introduced in Chapter 3. In Sec. 4.2 we come to the actual focus of this chapter, the finite-frequency noise associated to the current flow through the hybrid quantum-dot system. The results presented in this chapter are already published in Reference [151]. Throughout this chapter all figures will focus on the positive frequency part of the spectrum only, since we consider a symmetrized noise spectrum. 


\subsection{Single-level quantum dot coupled to normal- conducting leads}

In this section we present results for the current and the noise through a single-level quantum dot coupled to two normal-conducting leads. We apply the diagrammatic real-time approach for noise introduced in Chapter 3 and consider a non-interacting system as well as finite on-site Coulomb interaction on the quantum dot.

The finite-frequency noise of a single-level quantum dot coupled to normalconducting lead has been studied in Refs. 29, 33, 36, 37, 40,43,45 using various

methods. It turns out that the presentation of the known results of the finitefrequency noise for a single-resonant level, see Refs. 229, 33, 36, 37, 40, 43, 45, is helpful for the understanding of the more complex results for the interacting proximized dot studied in Sec. 4.2. As we will discuss later in several cases, the effect of the differently coupled Andreev levels can be mimicked by considering asymmetric coupling of the dot to the two normal-conducting leads. Note, in this section we consider the total symmetrized current, namely $\left(I_{\mathrm{L}}-I_{\mathrm{R}}\right) / 2$ and its noise. While in the next section, when discussing the hybrid system of a quantum dot coupled to a normal and a superconducting lead, we take into account current and noise only in the normal-conducting lead.

The model of the system has been introduced in Sec. 3.1. The reduced density matrix of the system has the form

$$
\boldsymbol{P}=\left(\begin{array}{cccc}
P_{0}^{0} & 0 & 0 & 0 \\
0 & P_{\uparrow}^{\uparrow} & P_{\downarrow}^{\uparrow} & 0 \\
0 & P_{\uparrow}^{\downarrow} & P_{\downarrow}^{\downarrow} & 0 \\
0 & 0 & 0 & P_{d}^{d}
\end{array}\right)
$$

where the diagonal elements can be interpreted as the probabilities to find the dot empty $P_{0}^{0} \equiv P_{0}$, singly occupied, $P_{\sigma}^{\sigma} \equiv P_{\sigma}$, or doubly occupied, $P_{\mathrm{d}}^{\mathrm{d}} \equiv$ $P_{\mathrm{d}}$. The off-diagonal elements describe the superposition of two eigenstates. These can be disregarded in the case of a single-level quantum dot coupled 
to normal-conducting leads, because its time evolution decouples from the one of the diagonal elements due to spin-conserving tunneling.

\subsubsection{Non-interacting single-level quantum dot}

In this section, we will present results for the finite-frequency noise of a non-interacting single-level quantum dot. Figure 4.1 shows a energy landscape of the system under consideration. The finite-frequency noise of a non-interacting single-level quantum dot has been calculated by means of various approaches, see Refs. $29,33,36,37,40,43,45$. In the non-interacting case the finite-frequency noise spectrum can be obtained exactly using the scattering matrix approach [32]. The scattering matrix formalism for noise and results are included in Appendix A. The discussion of the finite-frequency noise is divided into different bias and frequency regimes. First, we present results for the unidirectional transport regime, where back tunneling to the reservoir is blocked due to the applied bias voltage. Next, we discuss the regime where the bias is such that the level is outside the bias window and lastly we present the regime of a finite applied bias voltage, where also back tunneling to the reservoir is allowed. It turns out that in this regime asymmetric coupling of the dot to the leads shows the weight of the different noise contributions on the total noise.

\section{Unidirectional transport regime}

We assume throughout this section a symmetric bias between left and right reservoir, namely $\mu_{\mathrm{L}}=-\mu_{\mathrm{R}}=V / 2$. In the unidirectional transport regime, when the applied bias voltage is such that $\epsilon<V / 2$, the current is given by

$$
I_{\text {uni }}=\frac{2 \Gamma_{\mathrm{L}} \Gamma_{\mathrm{R}}}{\Gamma},
$$

with $\Gamma=\Gamma_{\mathrm{L}}+\Gamma_{\mathrm{R}}$. The finite-frequency noise in this unidirectional transport regime, when also $V>\omega$ is fulfilled, is given by

$$
S_{\text {uni }}(\omega)=I_{\text {uni }}\left[1+\frac{\left(\Gamma_{\mathrm{L}}-\Gamma_{\mathrm{R}}\right)^{2}}{\Gamma^{2}+\omega^{2}}\right] .
$$




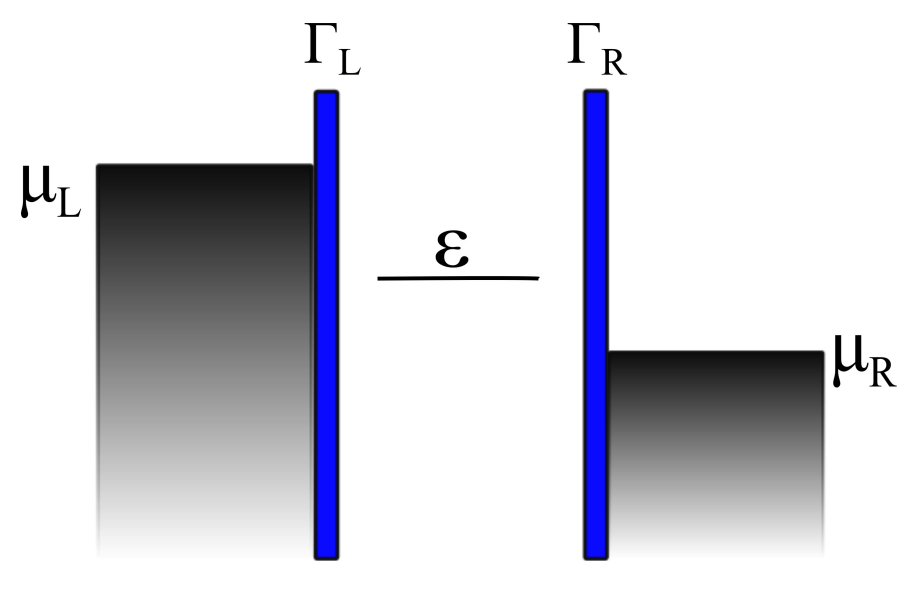

Figure 4.1: Sketch of the energy landscape of a noninteracting single-level quantum dot (with level energy $\epsilon$ ) coupled to normal-conducting leads (with electrochemical potentials $\mu_{\mathrm{L}}, \mu_{\mathrm{R}}$ and $\mu_{\mathrm{L}}-\mu_{\mathrm{R}}=V$ ). The coupling strengths to the leads are given by $\Gamma_{\mathrm{L}}$ and $\Gamma_{\mathrm{R}}$.

The noise shows a Lorentzian dependence on the noise frequency $\omega[32,34$. For a symmetric coupling of the dot to the normal conducting leads $\Gamma_{L}=\Gamma_{R}$, the noise equals $\Gamma / 2$ and is hence independent of the noise frequency. In this case the system has no bottleneck for transport and electrons tunnel independently on the quantum dot. Hence the noise shows no Lorentzian dependence.

In the unidirectional transport regime, where the applied bias voltage is the largest energy scale, we only access the shot noise regime.

\section{Low- and finite-bias regime}

In the regime of zero and low-bias, when the energy level of the dot is outside the bias window and transport is blocked, the dominant noise contribution is quantum noise, since $\omega>\mu_{N}, k_{B} T, \Gamma$. Shot noise is not present and thermal noise is only contributing for low frequencies and it is cut off at $\omega=k_{B} T$. Hence, this bias regime is suitable to study quantum noise effects in the noise spectrum. Quantum noise, which arises from zero point fluctuations in the 
device, gives a direct access to the internal dynamics of the system and makes new transport channels visible, which lead to steps in the noise spectrum. This behavior is typical for the high frequency noise of a system where the transport is blocked. It is a measure of the ability of the system to absorb or emit a certain energy $\omega$ [24].

In order to get an insight into the parameters controlling the height of the steps occurring in the quantum noise regime, we here analyze the highfrequency noise spectrum in the regime of low and finite bias, where quantum noise is dominant. For $\omega \gg \Gamma$ the noise in the high-frequency regime is found to be given by

$$
\begin{aligned}
S_{\text {fin }}(\omega) & =\frac{1}{2} \frac{\Gamma_{\mathrm{L}}^{2}}{\Gamma}\left[f_{\mathrm{L}}^{+}(\epsilon) f_{\mathrm{L}}^{-}(\epsilon+\omega)+f_{\mathrm{L}}^{+}(\epsilon-\omega) f_{\mathrm{L}}^{-}(\epsilon)\right] \\
& +\frac{1}{2} \frac{\Gamma_{\mathrm{R}}^{2}}{\Gamma}\left[f_{\mathrm{R}}^{+}(\epsilon) f_{\mathrm{R}}^{-}(\epsilon+\omega)+f_{\mathrm{R}}^{+}(\epsilon-\omega) f_{\mathrm{R}}^{-}(\epsilon)\right] \\
& +\frac{1}{2} \frac{\Gamma_{\mathrm{L}} \Gamma_{\mathrm{R}}}{\Gamma}\left[f_{\mathrm{L}}^{+}(\epsilon+\omega) f_{\mathrm{R}}^{-}(\epsilon)+f_{\mathrm{L}}^{+}(\epsilon) f_{\mathrm{R}}^{-}(\epsilon-\omega)\right] \\
& +\frac{1}{2} \frac{\Gamma_{\mathrm{L}} \Gamma_{\mathrm{R}}}{\Gamma}\left[f_{\mathrm{R}}^{+}(\epsilon) f_{\mathrm{L}}^{-}(\epsilon+\omega)+f_{\mathrm{R}}^{+}(\epsilon-\omega) f_{\mathrm{L}}^{-}(\epsilon)\right] \\
& +\omega \rightarrow-\omega,
\end{aligned}
$$

with the Fermi function $f_{\alpha}^{+}(\omega)=1 /\left(1+e^{\left(\omega-\mu_{\alpha}\right) / k_{\mathrm{B}} T}\right)$ for the two leads $\alpha=\mathrm{L}, \mathrm{R}$ and $f_{\alpha}^{-}(\omega)=1-f_{\alpha}^{+}(\omega)$. While the first two contributions result from correlations in the same lead, the latter two are related to correlations between different leads. In the following we will analyse the noise systematically for the different bias regimes and investigate the effect of an asymmetric coupling to the reservoirs $\left(\Gamma_{\mathrm{L}} \neq \Gamma_{\mathrm{R}}\right)$ on the noise spectrum.

\section{Zero bias}

In the limit of $V \rightarrow 0$, shot noise is negligible. Thermal noise, which is generally cut off at $\omega=k_{\mathrm{B}} T$, is here suppressed due to the fact that the energy of the level is $\epsilon \neq 0$. This means that the conductance is strongly suppressed. Hence quantum noise is dominant in this regime. 


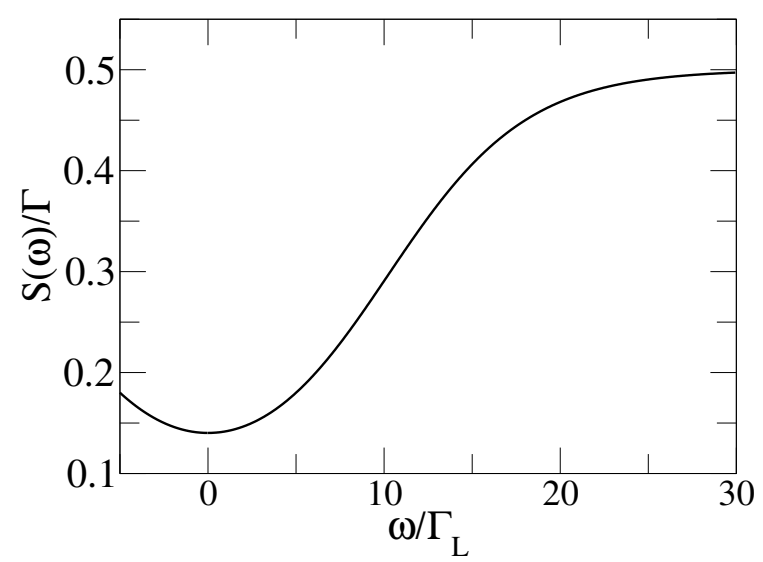

Figure 4.2: Finite-frequency noise $S(\omega)$ for a quantum dot coupled to normal leads in units of $\Gamma$ in the zero-bias regime $(\mathrm{V}=0)$ with $\Gamma_{\mathrm{L}}=\Gamma_{\mathrm{R}}, \epsilon=10 \Gamma_{\mathrm{L}}$, $k_{\mathrm{B}} T=4 \Gamma_{\mathrm{L}}$.

The noise spectrum, Fig. 4.2 , has one step at $\omega=|\epsilon|$. Since at zero bias all factors in Eq. (4.4) containing Fermi functions are equal, the step height is given by $\Gamma / 2$. An asymmetric coupling to the leads does hence not influence the shape of the noise spectrum.

\section{Low bias, $\epsilon>V / 2$}

When a finite transport voltage is applied, but the transport level is outside the bias window, quantum noise is still the dominant noise contribution and the noise spectrum exhibits two steps.

For the situation shown in Fig. 4.3. when $\epsilon>\mu_{\mathrm{L}}>\mu_{\mathrm{R}}$, the quantum dot is unoccupied in the stationary regime and all factors in Eq. (4.4) containing $f_{\alpha}^{+}(\epsilon)$ are zero. Then the first step stems from the contributions of the first and the third term of Eq. (4.4) for which the factor containing Fermi functions is equal. It occurs at $\omega=\left|\epsilon-\mu_{\mathrm{L}}\right|$ when the excitation of the dot from the left lead becomes visible and it has the height $\Gamma_{\mathrm{L}} / 2$. Analogously, the second step at $\omega=\left|\epsilon-\mu_{\mathrm{R}}\right|$ has height $\Gamma_{\mathrm{R}} / 2$. In both places an increase of the noise is observed as long as the dot level is outside the bias window, because in both 


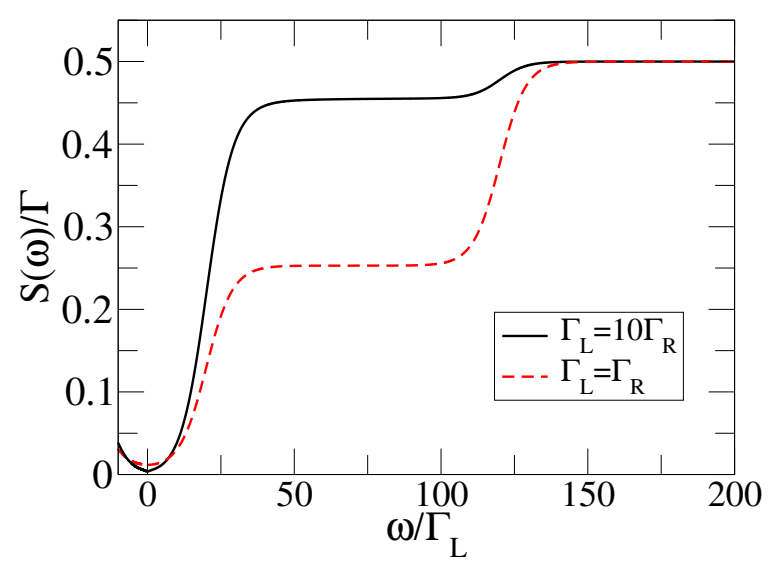

Figure 4.3: Finite-frequency noise $S(\omega)$ for a quantum dot coupled to normal leads in units of $\Gamma$ in the low-bias regime for different coupling strengths with $\epsilon=70 \Gamma_{\mathrm{L}}, k_{\mathrm{B}} T=4.5 \Gamma_{\mathrm{L}}$ and $V=100 \Gamma_{\mathrm{L}}$.

cases the effect of an otherwise blocked transport channel becomes visible. Fig. 4.3 shows two noise spectra, for a symmetrically coupled quantum dot $\Gamma_{\mathrm{L}}=\Gamma_{\mathrm{R}}$ (red dashed line) and an asymmetrically coupled $\operatorname{dot} \Gamma_{\mathrm{L}}>\Gamma_{\mathrm{R}}$ (black solid line).

Finite bias, $\epsilon<V / 2$

We finally consider the case, where the energy level lies inside the bias window and shot noise as well as quantum noise is present. When choosing asymmetric coupling to the leads, we find a situation which can be compared to the proximized quantum dot with finite detuning as discussed in Sec. 4.2.

In Fig. 4.4 the noise is displayed for a situation where the left lead is coupled more strongly to the quantum dot $\Gamma_{L}=10 \Gamma_{R}$. The Lorentzian behaviour of the low-frequency noise, $\omega \ll\left(\mu_{\mathrm{L}}-\epsilon\right)$, is described with the expression given in Eq. 4.3). Furthermore, steps occur at $\omega=\left|\mu_{\mathrm{L}}-\epsilon\right|$ and $\omega=\left|\mu_{\mathrm{R}}-\epsilon\right|$. The first step at $\omega=\left|\mu_{\mathrm{L}}-\epsilon\right|$ increases the noise. At this frequency back-tunnelling of an electron to the strongly coupled left 


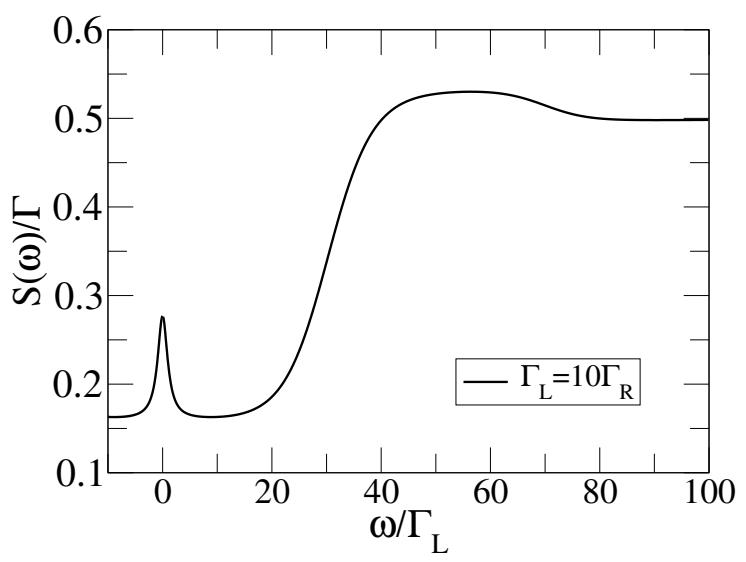

Figure 4.4: Finite-frequency noise $S(\omega)$ for a quantum dot coupled to normal leads in units of $\Gamma$ in the finite bias regime with asymmetric coupling $\Gamma_{\mathrm{L}}=$ $10 \Gamma_{\mathrm{R}}, \epsilon=20 \Gamma_{\mathrm{L}}, k_{\mathrm{B}} T=4 \Gamma_{\mathrm{L}}$ and $V=100 \Gamma_{\mathrm{L}}$.

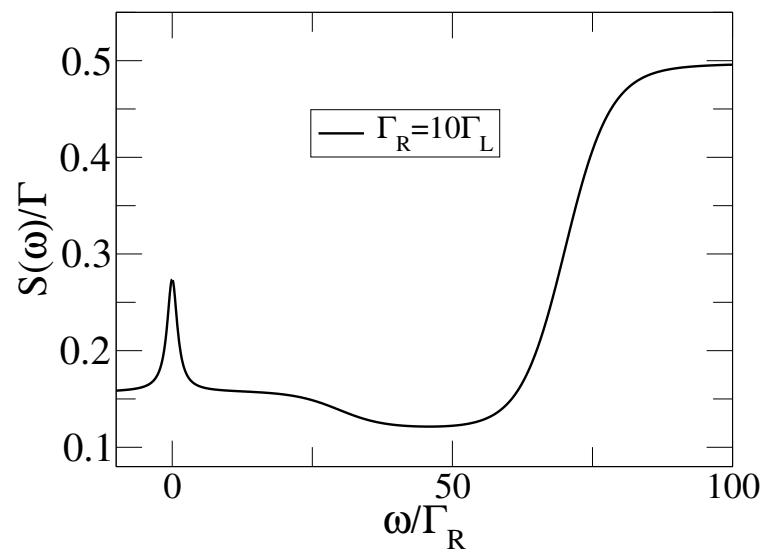

Figure 4.5: Finite-frequency noise $S(\omega)$ for a quantum dot coupled to normal leads in units of $\Gamma$ with in the finite bias regime with inverted asymmetry $\Gamma_{\mathrm{R}}=10 \Gamma_{\mathrm{L}}, \epsilon=20 \Gamma_{\mathrm{R}}, k_{\mathrm{B}} T=4 \Gamma_{\mathrm{R}}$ and $V=100 \Gamma_{\mathrm{R}}$. 
lead, emptying the quantum dot, becomes visible. Its height is given by $\Gamma_{L}\left(\Gamma_{L}-\Gamma_{R}\right) / 2\left(\Gamma_{R}+\Gamma_{L}\right)$. The second step at $\omega=\left|\epsilon-\mu_{R}\right|$, which occurs when an electron can tunnel back onto the dot from the right lead, results in a decrease of the noise. Its depth is given by $-\Gamma_{R}\left(\Gamma_{R}-\Gamma_{L}\right) / 2\left(\Gamma_{R}+\Gamma_{L}\right)$. The high frequency noise is again given by $\Gamma / 2$.

Whether a step in the noise leads to an increase or a decrease of the total noise depends on the coupling strength of the different excitations. The reason for this is that, in the regime where the level is in the bias window, the part of the noise stemming from correlations in the same lead increases at the frequencies equal to the excitation energies with an amount given by the square of the respective coupling strength, while the noise due to correlations between different leads decreases by an amount which is always proportional to $\Gamma_{\mathrm{L}} \Gamma_{\mathrm{R}}$. This can be directly read from Eq. (4.4). The step changes direction depending on the magnitude of the positive or negative contribution: Is the positive contribution multiplied by $\Gamma_{\alpha}^{2}$ larger the step increases, while it decreases if the negative contribution multiplied by $\Gamma_{L} \Gamma_{R}$ dominates.

Fig. 4.5 shows the noise for the same applied bias voltage but with the right reservoir coupled stronger than the left reservoir, $\Gamma_{R}=10 \Gamma_{L}$. The noise spectrum therefore shows a reversed order of the steps with respect to the result shown in Fig. 4.4, leading to an occurrence of troughs rather than plateaus in the noise.

Figure 4.6 shows the noise for a symmetrically coupled quantum dot $\left(\Gamma_{\mathrm{L}}=\Gamma_{\mathrm{R}}\right)$ for the case when the parameters have been chosen such that the probabilities of the dot being empty or singly occupied are equal. The noise spectrum shows no quantum noise steps at $\omega=\left|\epsilon-\mu_{\mathrm{L} / \mathrm{R}}\right|$ due to an almost complete compensation of the noise stemming from correlations in the same lead and correlations between the two leads.

The sum of these contributions to the noise leads to the shallow dip structure in the symmetrized noise spectrum as displayed in Fig. 4.6. For frequencies larger than the bias voltage, the noise takes again the value $\Gamma / 2$. 


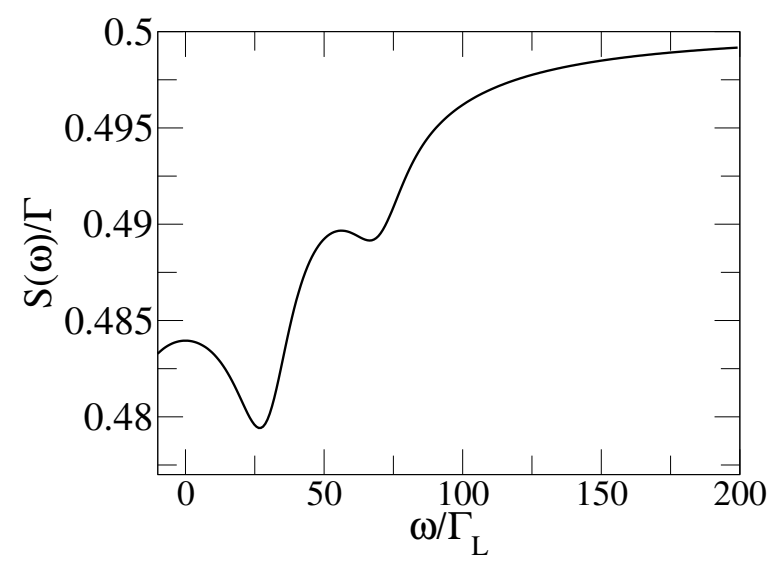

Figure 4.6: Finite-frequency noise $S(\omega)$ for a quantum dot coupled to normal leads in units of $\Gamma$ in the finite bias regime with symmetric coupling $\Gamma_{L}=\Gamma_{R}$, and $\epsilon=20 \Gamma_{\mathrm{L}}, k_{\mathrm{B}} T=4 \Gamma_{\mathrm{L}}$ and $V=100 \Gamma_{\mathrm{L}}$.

\subsubsection{Noise spectrum for a finite on-site Coulomb in- teraction}

The diagrammatic real-time approach for noise also allows the inclusion of a finite Coulomb repulsion on the dot. If we consider also a finite Coulomb interaction, the quantum dot has two transport levels with energies $\epsilon$ and $\epsilon+U$. Consequently, the noise spectrum will exhibit four steps in the quantum noise regime, namely at $\omega=\left|\epsilon-\mu_{L / R}\right|$ and $\omega=\left|\epsilon+U-\mu_{L / R}\right|$. The noise spectrum shows a mixture of the features discussed for the $U=0$ case depending on the position of the transport levels with respect to the bias window. Figure 4.7 displays the situation where both addition energies, $\epsilon$ and $\epsilon+U$, are within the transport window. Figure 4.7 (a) shows the finite-frequency noise spectrum for a symmetrically coupled quantum dot $\Gamma_{\mathrm{L}}=\Gamma_{\mathrm{R}}$. Analogous to Fig. 4.6 the spectrum shows no quantum noise steps at $\omega=\left|\epsilon-\mu_{L / R}\right|$ and $\omega=\left|\epsilon+U-\mu_{L / R}\right|$ due to an almost complete compensation of the noise stemming from correlations in the same lead and correlations between the two leads. Figure 4.7 (b) displays the noise spectrum for the asymmetrically coupled quantum $\operatorname{dot}\left(\Gamma_{\mathrm{L}}=5 \Gamma_{\mathrm{R}}\right)$ with quantum noise 
steps similar to Fig. 4.4 with two additional steps at $\omega=\left|\mu_{\mathrm{L}}-(\epsilon+U)\right|$ and $\omega=\left|\epsilon+U-\mu_{\mathrm{R}}\right|$, respectively. The sign of the steps, whether the total noise is increasing or decreasing, depends on the coupling strength of the different excitations.

Figure 4.8 shows the noise spectrum for the case that the transport level $\epsilon+U$ lies outside the bias window for (a) a symmetrically coupled quantum $\operatorname{dot}\left(\Gamma_{\mathrm{L}}=\Gamma_{\mathrm{R}}\right)$ and (b) for an asymmetric coupling to the normal-conducting leads $\left(\Gamma_{L}=5 \Gamma_{R}\right)$. In this case the noise spectrum shows an overlap of the previously discussed features. 

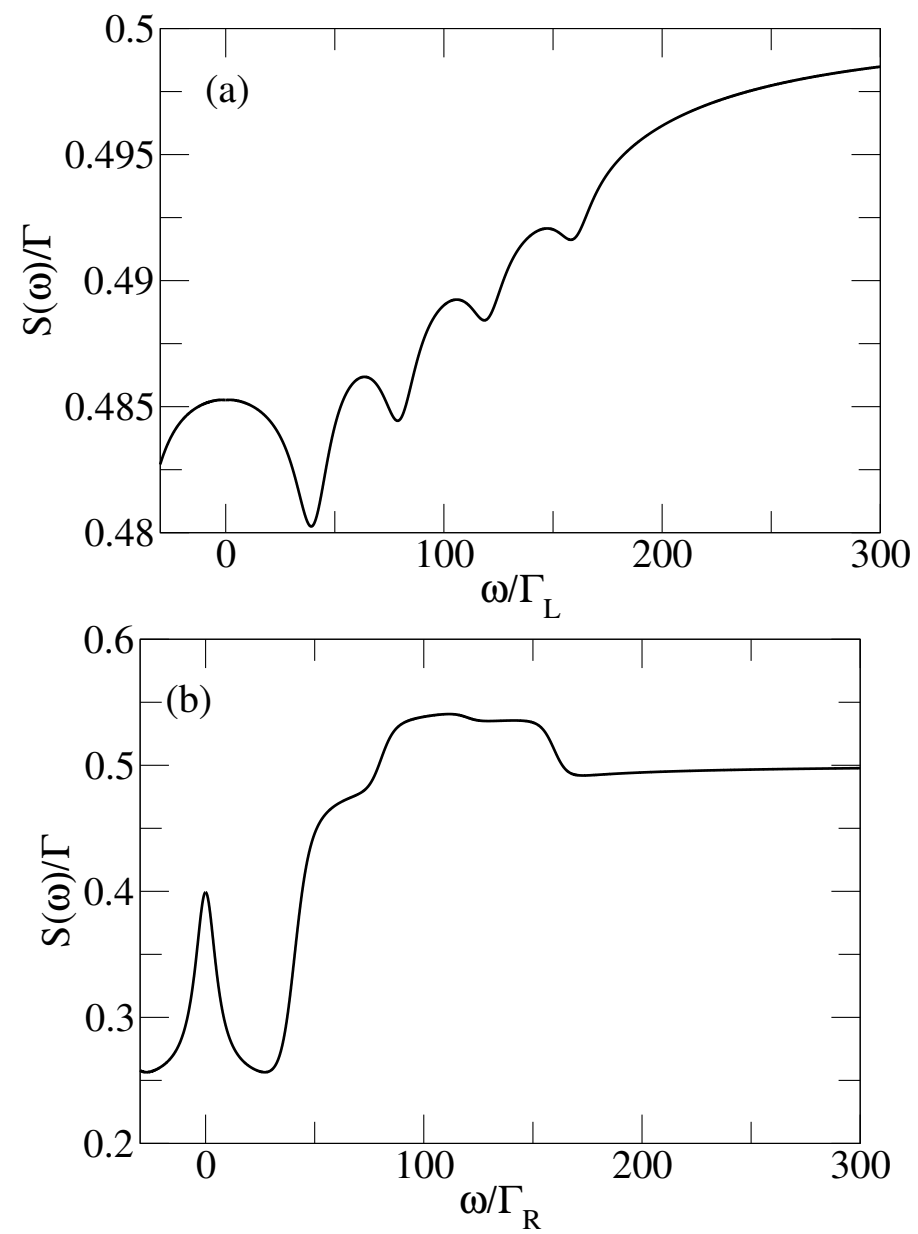

Figure 4.7: Finite-frequency noise $S(\omega)$ in units of $\Gamma$ for $U=40 \Gamma_{\mathrm{R}}, \epsilon=20 \Gamma_{\mathrm{R}}$, $k_{B} T=3 \Gamma_{\mathrm{R}}$ and $V=200 \Gamma_{\mathrm{R}}$, (a) $\Gamma_{\mathrm{L}}=\Gamma_{\mathrm{R}}$ and (b) $\Gamma_{\mathrm{L}}=5 \Gamma_{\mathrm{R}}$. 

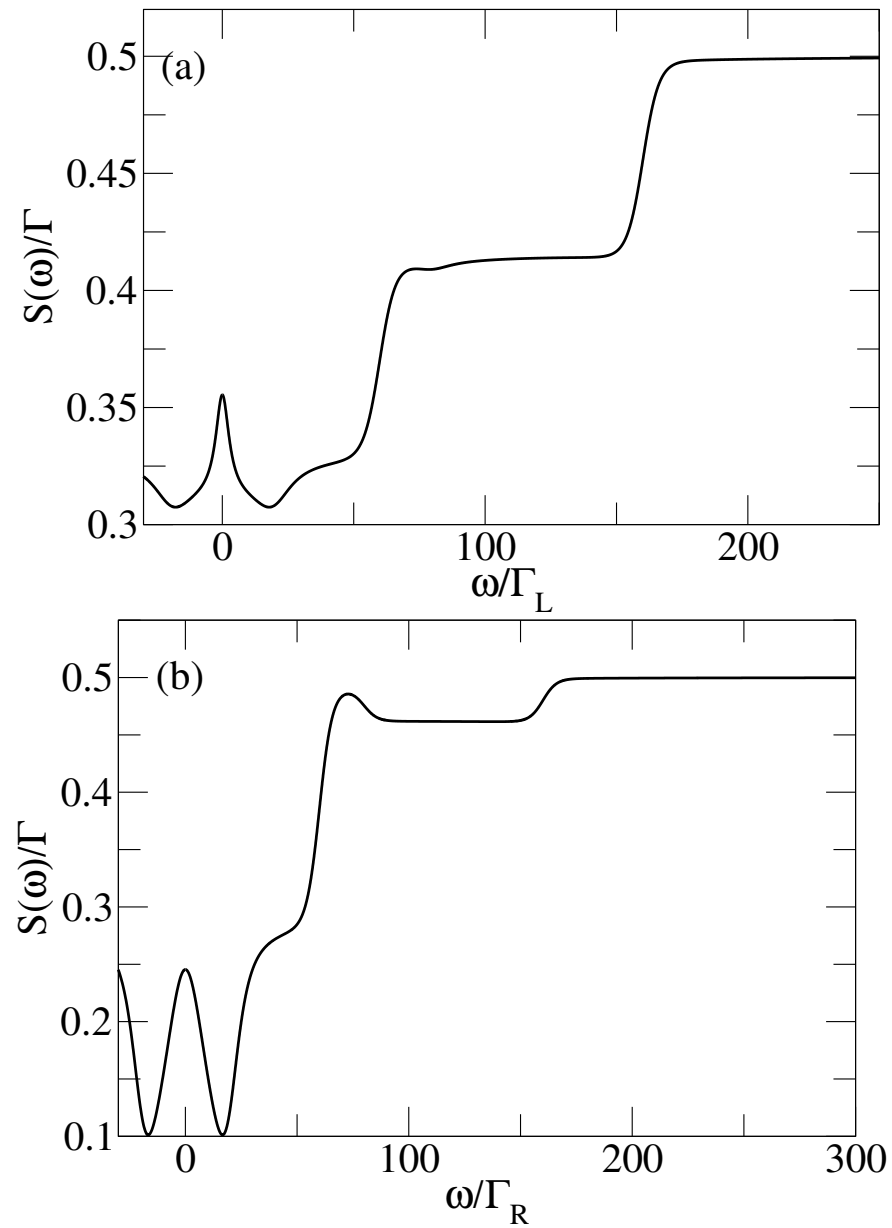

Figure 4.8: Finite-frequency noise $S(\omega)$ in units of $\Gamma$ for $U=80 \Gamma_{\mathrm{R}}, \epsilon=30 \Gamma_{\mathrm{R}}$, $k_{B} T=3 \Gamma_{\mathrm{R}}$ and $V=100 \Gamma_{\mathrm{R}}$, (a) $\Gamma_{\mathrm{L}}=\Gamma_{\mathrm{R}}$ and $(\mathrm{b}) \Gamma_{\mathrm{L}}=5 \Gamma_{\mathrm{R}}$. 


\subsection{Quantum dot tunnel-coupled to normal and superconducting leads}

We come now to the main focus of this Chapter, the discussion of the finitefrequency noise through a hybrid quantum-dot system. The model has been introduced in Sec. 3.1 with Fig. 3.2 showing the effective dot-superconductor subsystem weakly coupled to a normal conducting lead which acts as a bath. When discussing the results of this section, we will always assume $\Gamma_{N} \ll \Gamma_{S}$.

The full system is represented by a density matrix describing the normalconducting lead (which has many degrees of freedom but is non-interacting) coupled to the interacting quantum dot proximized by the superconducting condensate (the latter having just a few degrees of freedom). Since we are not interested in the degrees of freedom of the normal-conducting reservoir we trace them out, making use of Wick's theorem. We are then left with an effective description by means of the reduced density matrix of the quantum dot proximized by the superconductor. This reduced density matrix has the form

$$
\boldsymbol{P}=\left(\begin{array}{cccc}
P_{+}^{+} & P_{-}^{+} & 0 & 0 \\
P_{+}^{-} & P_{-}^{-} & 0 & 0 \\
0 & 0 & P_{\uparrow}^{\uparrow} & P_{\downarrow}^{\uparrow} \\
0 & 0 & P_{\uparrow}^{\downarrow} & P_{\downarrow}^{\downarrow}
\end{array}\right),
$$

where the diagonal elements are the probabilities to find the dot singly occupied, $P_{\sigma}^{\sigma} \equiv P_{\sigma}$, or in a BCS-like state, $P_{+}^{+} \equiv P_{+}$or $P_{-}^{-} \equiv P_{-}$. The off-diagonal elements, which we refer to as the coherences, describe coherent superposition of two eigenstates of the proximized dot. Coherent superpositions of eigenstates with even and odd numbers of electrons are not allowed due to the univalence superselection rule [152]. These coherences are zero in the reduced density matrix Eq. 4.5. Importantly, the time evolution of the coherences between states of single occupation $\left(P_{\downarrow}^{\uparrow}\right.$ and $\left.P_{\uparrow}^{\downarrow}\right)$ decouples from the one of the diagonal elements due to spin-conserving tunnelling and these coherences will hence be disregarded in the following. In contrast, in order 
to fully describe the short-time dynamics of the system, it is necessary to consider the off-diagonal elements in the reduced density matrix between the Andreev bound states, $P_{-}^{+}$and $P_{+}^{-}$. It turns out that these become important for the finite-frequency noise at frequencies $\omega \sim \pm\left(\epsilon_{+}-\epsilon_{-}\right)$.

\subsection{Andreev current}

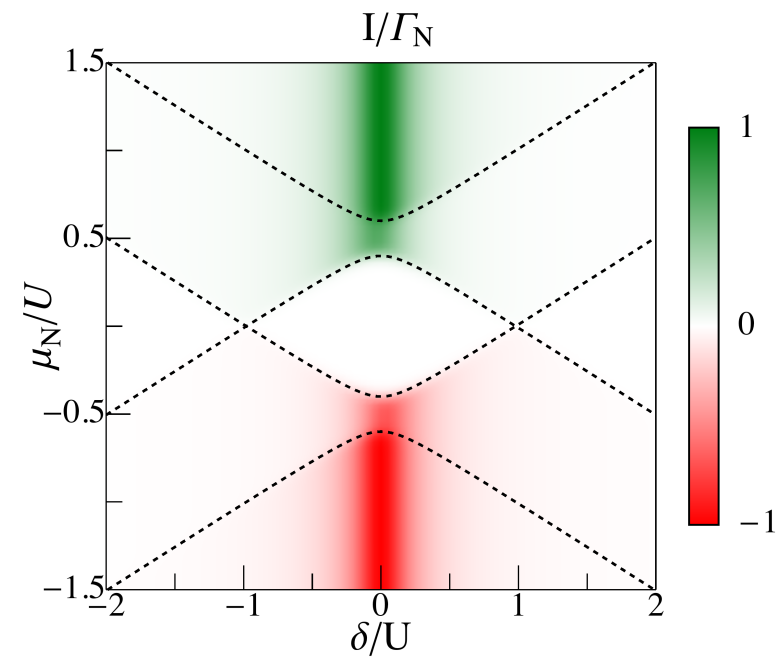

Figure 4.9: Density plot of the Andreev current as a function of the detuning $\delta$ and the chemical potential of the normal lead $\mu_{\mathrm{N}}$ both in units of the Coulomb interaction strength $U$. The other parameters are $\Gamma_{\mathrm{S}}=0.2 U$, $\Gamma_{\mathrm{N}}=0.002 U$ and $k_{\mathrm{B}} T=0.02 U$.

Before discussing the finite-frequency current noise, we give a brief overview over the properties of the Andreev current. When a finite bias voltage is applied across the quantum dot with one superconducting and one normalconducting lead, a so called Andreev current flows across the structure, which is due to Cooper-pair tunnelling between the quantum dot and the super- 
conductor caused by Andreev reflection processes [49,153].

We determine the current through the single-level quantum dot by using Eq. (3.26), and show the result in Fig. 4.9 as a function of the chemical potential of the normal lead, $\mu_{\mathrm{N}}$, and the detuning, $\delta$. The current is largest when superconducting correlations on the dot are strong and it furthermore shows features at the Andreev addition energies.

The Andreev addition energies (dashed lines in Fig. 4.9) are symmetric around zero bias voltage, $\mu_{\mathrm{N}}=0$, and with respect to zero detuning, $\delta=0$. In the region around zero bias voltage the system is mainly in one of the singly-occupied states, $|\sigma\rangle$, since the charging energy suppresses transitions from $|\sigma\rangle$ to the $| \pm\rangle$ states. The Andreev current is thus zero. Only when the bias voltage is large enough, such that one of the conditions $\mu_{\mathrm{N}} \gtrsim E_{+-}$ or $\mu_{\mathrm{N}} \lesssim E_{-+}$is fulfilled, the quantum dot has a finite probability to be either empty or doubly occupied and the Andreev current sets in. A further increase of the Andreev current is observed, when also the other two addition energies, $E_{++}$and $E_{--}$, enter the bias window.

Outside the region where the current is suppressed due to the charging energy, the current is largest for $\delta \sim 0$, the regime of strongest superconducting correlation. We obtain a simple analytic result for the Andreev current in the unidirectional transport regime, namely when $\mu_{\mathrm{N}} \gg E_{+,+}$, where the applied bias voltage to the normal conducting lead is much larger than all other energy scales in the system apart from the superconducting gap $\Delta$,

$$
I_{\text {uni }}=\Gamma_{\mathrm{N}} \frac{\Gamma_{\mathrm{S}}^{2}}{4 \epsilon_{\mathrm{A}}^{2}}
$$

As required, this result matches the current displayed in Fig. 4.9, for $\mu_{\mathrm{N}} \gg$ $E_{++}$. Indeed, the current in the unidirectional regime, Eq. (4.6), is maximal for $|\delta| \ll \Gamma_{\mathrm{S}}$, namely when $2 \epsilon_{\mathrm{A}}$, the splitting between the ABSs, is minimal and just given by the coupling strength to the superconducting lead $\Gamma_{\mathrm{S}}$. In this situation, the empty $|0\rangle$ and doubly-occupied state $|d\rangle$ are nearly degenerate and hence the mixing between them is maximal (the proximity effect is on resonance). If the detuning $\delta$ becomes large, i.e. $|\delta| \gg \Gamma_{\mathrm{S}}$, the 
superconducting correlations on the dot are almost zero (the proximity effect is off-resonance) and the Andreev current goes to zero as shown in Fig. 4.9.

The value of the Andreev addition energies can however only roughly be extracted from the current. But we will show in Secs. 4.4.1, 4.4.2 and 4.4.3 that they lead to sharp features in the noise spectrum.

\subsection{Results for the finite-frequency noise}

This section deals with the key results of this chapter, the finite-frequency noise associated with the tunnelling current flowing through the hybrid quantumdot system, which we calculate based on the diagrammatic real-time approach introduced in the previous Chapter 3

The discussion of the finite-frequency noise is divided into three parts: the unidirectional transport regime, where the applied bias voltage $\mu_{\mathrm{N}}$ is chosen such that no back tunnelling to the normal lead is allowed, the finite bias regime, where the applied bias voltage can be of the same order of the Andreev addition energies and the noise frequency, and the low-bias regime, where the current through the dot is suppressed. All regimes are shown to provide direct access to the internal dynamics of the system.

Depending on the applied bias voltage different frequency regimes of the noise are accessible. Table 4.1 gives an overview of the different types of noise depending on the characteristic energy scales of the system, the thermal energy $k_{\mathrm{B}} T$, the energy related to the noise frequency $\omega$, and the applied bias voltage $\mu_{\mathrm{N}}$, which we are going to discuss within the different parts of this section.

In the limit of zero frequency $(\omega \rightarrow 0)$ the noise spectrum exhibits the information of a long-time measurement. In this frequency range, the equilibrium thermal noise, due to thermal fluctuations in the occupation number of the leads, is dominant in the spectrum if $k_{\mathrm{B}} T \gg \mu_{\mathrm{N}}, \omega$. In contrast, the so-called non-equilibrium shot noise, which is due to charge quantization, is dominant for $\mu_{\mathrm{N}} \gg k_{\mathrm{B}} T, \omega$. 


\begin{tabular}{|c|c|c|c|}
\hline $\begin{array}{l}\text { Bias Frequency } \\
\text { regime }\end{array}$ & Low & Intermediate & $\begin{array}{l}\text { High, } \omega> \\
\mu_{\mathrm{N}}, k_{\mathrm{B}} T, \Gamma_{\mathrm{N}}\end{array}$ \\
\hline $\begin{array}{l}\text { Unidirectional, } \\
\mu_{\mathrm{N}} \text { largest en- } \\
\text { ergy scale }\end{array}$ & $\begin{array}{l}\omega \leq \Gamma_{\mathrm{N}} \\
\text { (shot } \\
\text { noise) } \\
\text { Sec. } 4.4 .1\end{array}$ & $\begin{array}{l}\omega>\Gamma_{\mathrm{N}} \\
\text { (shot } \\
\text { noise) } \\
\text { Sec. } 4.4 .1\end{array}$ & $\mathrm{~N} / \mathrm{A}$ \\
\hline Finite bias & $\begin{array}{l}\omega<\mu_{\mathrm{N}} \& \\
\omega \leq \\
k_{\mathrm{B}} T, \Gamma_{\mathrm{N}} \\
(\text { thermal } \\
\& \quad \text { shot } \\
\text { noise) } \\
\text { Sec. } 4.4 .3\end{array}$ & $\begin{array}{l}\mu_{\mathrm{N}}>\omega \& \\
\omega \\
k_{\mathrm{B}} T, \Gamma_{\mathrm{N}} \\
\text { (shot } \\
\text { noise) } \\
\text { Sec. } 4.4 .3\end{array}$ & $\begin{array}{l}\text { (quantum } \\
\text { noise) } \\
\text { Sec. } 4.4 .3\end{array}$ \\
\hline $\begin{array}{l}\text { Zero and low } \\
\text { bias }\end{array}$ & $\begin{array}{l}\omega \leq k_{\mathrm{B}} T \\
\text { (thermal } \\
\text { noise) } \\
\text { Sec. } 4.4 .2\end{array}$ & $\mathrm{~N} / \mathrm{A}$ & $\begin{array}{l}\text { (quantum } \\
\text { noise) } \\
\text { Sec. } 4.4 .2\end{array}$ \\
\hline
\end{tabular}

Table 4.1: Table sumarising different noise regimes depending on noise frequency (increasing from left to right) and applied bias voltage (decreasing from up to down). 
The quantum noise, which arises from zero-point fluctuations in the device, is dominant for high frequencies $\omega \gg k_{\mathrm{B}} T, \mu_{\mathrm{N}}$. It is a measure of the ability of the system to absorb or to emit a certain energy $\omega$ [24], and will therefore allow the visualization of transport processes which are enabled or blocked by energy absorption or emission.

Although we here consider the symmetrized noise, we refer to the regime of high frequencies $\omega \gg k_{\mathrm{B}} T, \mu_{\mathrm{N}}$ as quantum noise, as discussed e.g. in Ref. 63.

\subsubsection{Noise in the unidirectional transport regime}

We start our analysis with the unidirectional transport regime, where we set the chemical potential of the normal lead $\mu_{\mathrm{N}}$ to be much larger than all relevant energy scales of the system (apart from the superconducting gap $\Delta$ ). In particular, since $\mu_{\mathrm{N}} \gg E_{+,+}$, all Andreev levels are in the transport window and sufficiently far away from the chemical potential $\mu_{\mathrm{N}}$, thus allowing no back tunnelling from the dot into the normal conducting lead. A sketch of this situation is shown in Fig. 3.2.

The finite-frequency noise in the unidirectional transport regime is given by

$$
\begin{aligned}
\frac{S(\omega)}{2 I_{\mathrm{uni}}} & =1+\frac{\Gamma_{\mathrm{N}}^{2} \delta^{2}}{4 \epsilon_{\mathrm{A}}^{2}\left(\Gamma_{\mathrm{N}}^{2}+\omega^{2}\right)}-\frac{1}{2} \frac{\Gamma_{\mathrm{S}}^{2}}{4 \epsilon_{\mathrm{A}}^{2}} \times \\
& {\left[\frac{\Gamma_{\mathrm{N}}^{2}}{\Gamma_{\mathrm{N}}^{2}+\left(\omega-2 \epsilon_{\mathrm{A}}\right)^{2}}\left(1-\frac{\omega-2 \epsilon_{\mathrm{A}}}{\epsilon_{\mathrm{A}}}\right)+\frac{\Gamma_{\mathrm{N}}^{2}}{\Gamma_{\mathrm{N}}^{2}+\left(2 \epsilon_{\mathrm{A}}+\omega\right)^{2}}\left(1+\frac{\omega+2 \epsilon_{\mathrm{A}}}{\epsilon_{\mathrm{A}}}\right)\right] . }
\end{aligned}
$$

Fig. 4.10 shows the finite-frequency noise in units of $\Gamma_{\mathrm{N}}$ as a function of the noise frequency $\omega$. Two limiting cases are displayed, the one where the proximity effect on the dot is on resonance, $|\delta| \ll \Gamma_{\mathrm{S}}$ (red dashed line), and the one where the proximity effect on the dot is off resonance, $|\delta| \gg \Gamma_{\mathrm{S}}$ (blue solid line). In different frequency regimes, the spectrum shows sharp features, which depend on the strength of the detuning, $\delta$. In the following 
subsections we discuss first the low-frequency noise, followed by a discussion of the intermediate-frequency regime.

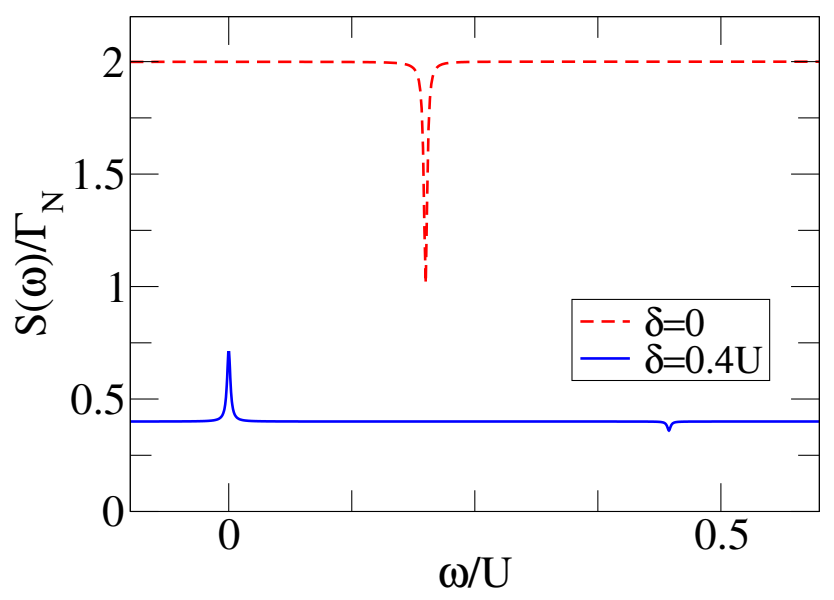

Figure 4.10: Finite-frequency noise $S(\omega)$ in the unidirectional transport regime for $\Gamma_{\mathrm{S}}=0.2 U, \Gamma_{\mathrm{N}}=0.002 U$ both for the case where the proximity effect is on resonance, $\delta=0$, and off resonance, $\delta=0.4 U$. The peaks and dips are located at $\omega=0$ and at $\omega= \pm 2 \epsilon_{\mathrm{A}}$, the oscillation frequency of the Cooper pairs.

\section{Low-frequency noise, $\omega \lesssim \Gamma_{\mathrm{N}}$}

The first part of Eq. (4.7) is the low-frequency contribution to the current noise,

$$
S(\omega)=2 \Gamma_{\mathrm{N}} \frac{\Gamma_{\mathrm{S}}^{2}}{4 \epsilon_{\mathrm{A}}^{2}}\left(1+\frac{\delta^{2} \Gamma_{\mathrm{N}}^{2}}{4 \epsilon_{\mathrm{A}}^{2}\left(\Gamma_{\mathrm{N}}^{2}+\omega^{2}\right)}\right) .
$$

It is indeed the only contribution to the noise, when the noise frequency is of the order of the coupling strength to the normal-conducting lead, $\omega \lesssim \Gamma_{\mathrm{N}}$. Note that in the low-frequency noise for unidirectional transport only shot noise is present.

On resonance $(\delta \approx 0)$, when the Andreev current is maximal in the high-bias regime, see Fig. 4.9, the noise is frequency independent and is 
given by two times the Andreev current $2 I_{\text {uni }}$, which in the limit of zerodetuning discussed here equals $2 \Gamma_{\mathrm{N}}$. This means that the noise equals the long-time measurement result $(\omega \rightarrow 0)$ over the whole low-frequency range. This effect has previously been discussed in Ref. [154]: if the proximity effect is on resonance, the superconducting correlations on the dot are maximal and Cooper pairs oscillate rapidly between dot and superconductor. This oscillation of Cooper pairs is only interrupted by single-electron tunnel events from the normal conducting lead to the dot. It is these independent charge injections which give rise to a Poissonian transfer of single electrons.

When the proximity effect is off resonance $\left(|\delta| \gg \Gamma_{\mathrm{S}}\right)$, the low-frequency noise can be approximated by

$$
S(\omega) \approx 2 \Gamma_{\mathrm{N}} \frac{\Gamma_{\mathrm{S}}^{2}}{\delta^{2}}\left(1+\frac{\Gamma_{\mathrm{N}}^{2}}{\Gamma_{\mathrm{N}}^{2}+\omega^{2}}\right) .
$$

The noise spectrum shows a Lorentzian dependence on the frequency $\omega$, as shown by the low-frequency contribution of Fig. 4.10 (solid blue line). This maximum has a width given by the coupling strength $\Gamma_{\mathrm{N}}$ and a height scaling with the magnitude of the Andreev current. Except for this maximum, the noise is overall suppressed with respect to the case on resonance. The reason for this is that in the unidirectional transport regime if $\delta \gg \Gamma_{\mathrm{S}}$, the Andreev current becomes negligibly small with increasing detuning, as depicted in Fig. 4.9.

This behaviour is similar to the case of a quantum dot with normal conducting leads only, 32, 34 as presented in the section 4.1.1. In this purely normal-conducting case the low-frequency noise shows a Lorentzian behaviour if the coupling to the two leads is asymmetric (similar to what we observe in the case of finite detuning in the hybrid system). The noise is frequency-independent, when the coupling to the leads is symmetric. However the constant is only half as big as in the hybrid case discussed here, due to the absence of Cooper pairs from the system. 


\section{Intermediate-frequency regime, $\omega \gg \Gamma_{N}$}

The remaining part of Eq. 4.7) carries the information of the noise in the intermediate-frequency regime, $\omega \gg \Gamma_{N}$, where the noise frequency becomes larger than the coupling strength to the normal-conducting lead. In this regime, the noise starts to reveal the internal dynamics of the quantum dot. Indeed, the finite-frequency noise shows resonance dips, whose position and size depend on the splitting of the Andreev bound states (equal to the frequency of coherent oscillation between the empty and doubly-occupied dot state [155]), $2 \epsilon_{\mathrm{A}}$, and hence on the strength of the proximity effect, see Fig. 4.10. Such dips are characteristic for the noise spectrum of multilevel quantum dots $35,37,40]$. Whenever the noise frequency is close to the difference between the energies of the dot resonances a dip can be found in the noise spectrum. Such dips must arise from a destructive interference between the quantum amplitudes for the transitions involving these resonances due to the ability of the system to absorb/emit a certain energy $\omega$ [35] similarly to the Fano effect 156. In the case of a hybrid quantum-dot system studied here, the dips arise from a coherent destructive interference between the $\mathrm{ABS}$, leading to features at $\omega= \pm\left|\epsilon_{+}-\epsilon_{-}\right|= \pm 2 \epsilon_{\mathrm{A}}$. In the following we describe the properties of these dips.

Similar to the noise-enhancing peak in the low-frequency regime, the shape of these noise-suppressing resonance dips is Lorentzian, see Eq. 4.7), with a width given by $\Gamma_{\mathrm{N}}$. The depth of the resonance dip depends on the strength of the proximity effect and is equal to $I_{\mathrm{uni}} \Gamma_{\mathrm{S}}^{2} / 4 \epsilon_{\mathrm{A}}^{2}$, the prefactor of the intermediate-frequency contribution in Eq. (4.7). The resonance dip becomes most prominent if the proximity effect is on resonance and its depth is maximally equal to $\Gamma_{\mathrm{N}}$ for $\delta \approx 0$. In contrast, the dip vanishes if the detuning $\delta$ becomes much larger than the coupling strength to the superconducting lead $\Gamma_{\mathrm{S}}$, namely when the superconducting correlations on the dot are almost zero, see Fig. 4.10. The resonance dip in the spectrum hence indicates the strength of the proximity effect. It is a signature of the coherent oscillation of Cooper pairs between dot and superconductor. 
For even higher frequencies, $\omega \gg 2 \epsilon_{\mathrm{A}}$, the noise in the unidirectional transport regime is given by two times the Andreev current, which depends strongly on the detuning $\delta$, see Figs. 4.9 and 4.10 .

In the unidirectional transport regime the noise spectrum can be used to extract the splitting of the ABS, but not the individual Andreev addition energies. In order to get the information of the excitation energies and the effective coupling strengths of the ABSs, back tunnelling to the normalconducting lead must also be allowed. Hence, in the next two subsections we will consider the regime where the electrochemical potential of the normal lead is not the largest energy scale any more and the frequency can become larger than the distance between the Andreev addition energies and the transport voltage, $\omega>\left|E_{ \pm, \pm}-\mu_{\mathrm{N}}\right|$. In this regime quantum noise can become the dominant contribution to noise.

\subsubsection{Zero and low bias regime, $\mu_{\mathbf{N}}<E_{+,-}$}

\section{Zero bias regime}

If the transport voltage of the normal lead goes to zero, $\mu_{\mathrm{N}} \rightarrow 0$, the dot is in the singly-occupied state $|\sigma\rangle$, see Fig. 4.11 (a) for a sketch of the energy landscape of the system. Hence the transport excitation energies $E_{ \pm, \pm}$are outside the bias window and transport is blocked, meaning that the Andreev current is zero as shown in Fig. 4.9. In this limit shot noise is negligible and quantum noise is dominant in the spectrum, since $\omega \gg k_{\mathrm{B}} T, \mu_{\mathrm{N}}$. Also the thermal noise, which is expected to be dominant at zero bias up to a noise frequency of $\omega=k_{B} T$, is here suppressed, since no dot excitation energy is close enough to the Fermi energy to allow for thermal excitations of the system.

We show the finite-frequency noise in this regime in Fig. 4.11 and we observe that the noise spectrum has steps at frequencies $\omega=\left|E_{ \pm, \pm}\right|$equal to the Andreev addition energies of the system. This behaviour is typical for the high-frequency noise of a system in which transport is blocked. At certain 

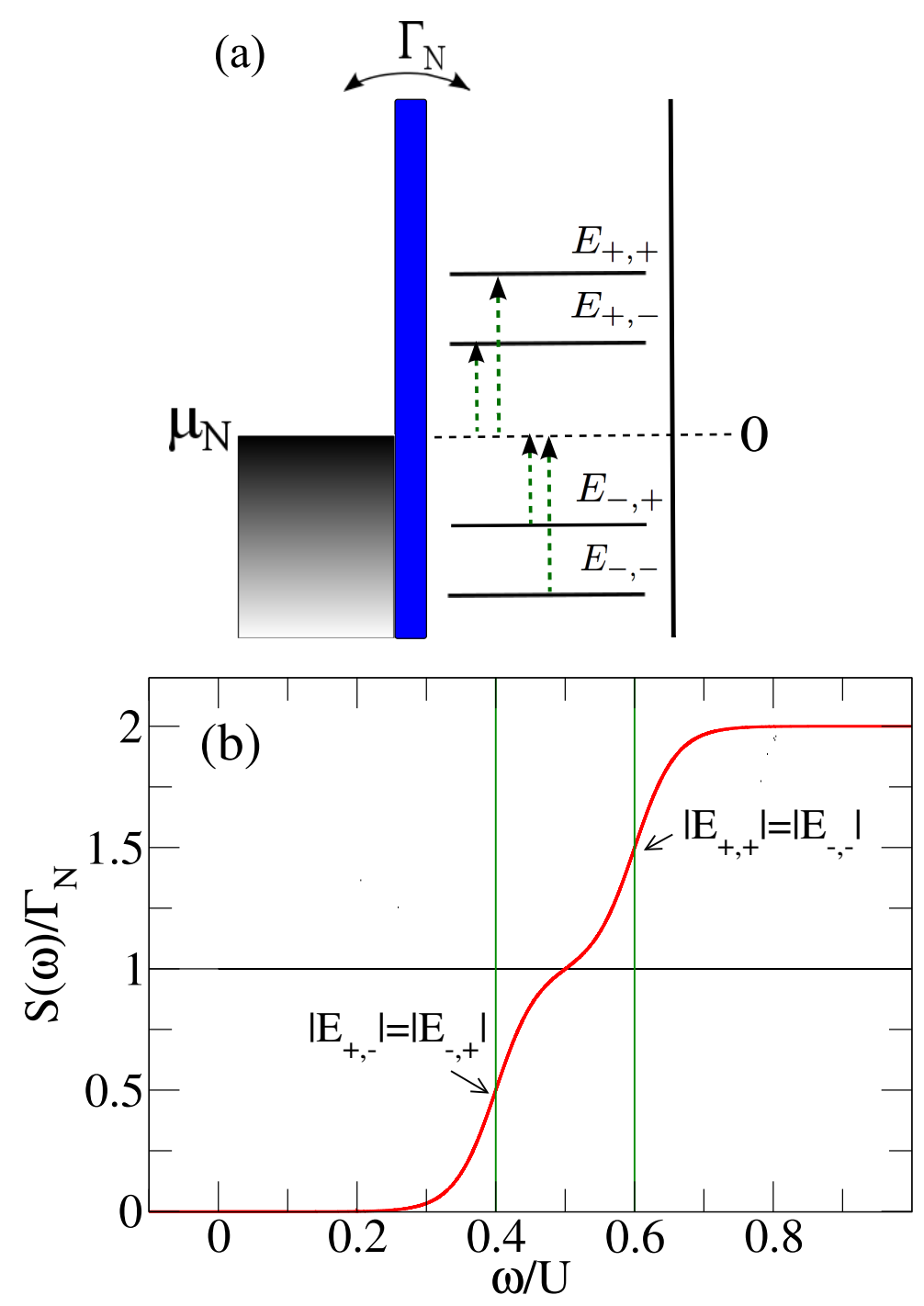

Figure 4.11: (a) Sketch of the energy landscape of the proximized single-level quantum dot for $\mu_{\mathrm{N}}=0$. (b) Finite-frequency noise $S(\omega)$ with $\Gamma_{\mathrm{N}}=0.0002 U$, $\Gamma_{\mathrm{S}}=0.2 U, \delta=0, k_{\mathrm{B}} T=0.03 U$; the spectrum has quantum noise steps at $\omega=E_{+,-}$and $\omega=E_{+,+}$indicated by the green vertical lines. 
noise frequencies the effect of new "noisy" channels becomes visible, leading to steps increasing the noise. The steps thus reflect the internal structure of the energy levels on the quantum dot. Here they occur at frequencies equal to the Andreev addition energies of the dot-superconductor subsystem as described in detail in the following. An analogy to the normal-conducting case with symmetrically and asymmetrically coupled leads can be found in the previous section 4.1 .1 .

In the limit $\mu_{\mathrm{N}} \rightarrow 0$, the noise is suppressed until the noise frequency is equal to the energy which is necessary to excite the dot from the singlyoccupied state $|\sigma\rangle$ to the $|-\rangle$ state, $\omega=\left|E_{+,-}\right|$. Equally, also the inverse process, namely the excitation from the ABS $|-\rangle$ into the singly-occupied state $|\sigma\rangle$ yields a contribution to the noise. It takes place at $\omega=\left|E_{-,+}\right|$. These excitation energies are however degenerate in the zero-bias limit, see Fig. 4.11 (a), and consequently only one step occurs at the noise frequency $\omega=\left|E_{-,+}\right|=\left|E_{+,-}\right|$. The step height is given by the sum of the respective effective coupling strengths, see Eqs. (3.7) and (3.8), $\Gamma_{\sigma \rightarrow-}+\Gamma_{-\rightarrow \sigma}=\Gamma_{\mathrm{N}} \cdot{ }^{1}$

A second step takes place at $\omega=\left|E_{+,+}\right|=\left|E_{-,-}\right|$, the energy necessary for the excitation between a singly-occupied state and the ABS $|+\rangle$. The height of the second step is again given by $\Gamma_{N}$, the sum of the effective coupling strengths, $\Gamma_{\sigma \rightarrow+}+\Gamma_{+\rightarrow \sigma}=\Gamma_{\mathrm{N}}$.

Consequently, when the noise frequency is larger than the energy which needs to be provided to excite between any of the singly-occupied states and the ABSs, the noise is constant and given by the sum of all four effective coupling strengths, $2 \Gamma_{\mathrm{N}}, 33,43,45$.

Low bias regime, $0<\mu_{\mathbf{N}}<E_{+,-}$

If a small (positive) bias voltage is applied to the normal-conducting lead, but the bias is still smaller than the energy $E_{+,-}$, necessary to excite from

\footnotetext{
${ }^{1}$ Note that we here consider a symmetrized noise spectrum. Therefore both contributions, independently on whether the excitation energy of a process is positive or negative, enter the noise spectrum with equal weight at positive frequencies.
} 

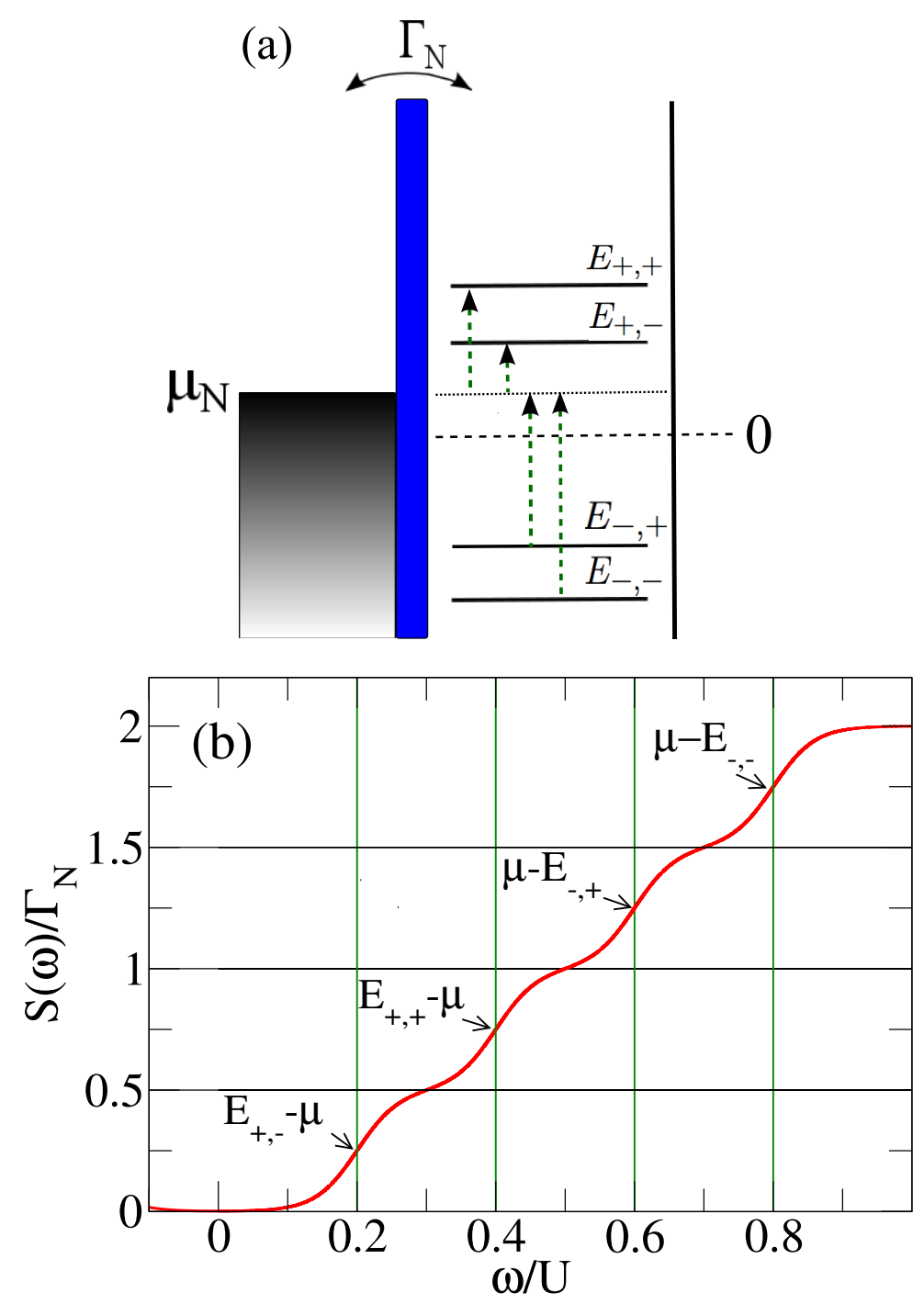

Figure 4.12: (a) Sketch of the energy landscape of the proximized singlelevel quantum dot for $\mu_{\mathrm{N}}=0.2 U$. (b) Finite-frequency noise $S(\omega)$ with $\Gamma_{\mathrm{N}}=0.0002 U, \Gamma_{\mathrm{S}}=0.2 U, \delta=0, k_{\mathrm{B}} T=0.03 U$. 

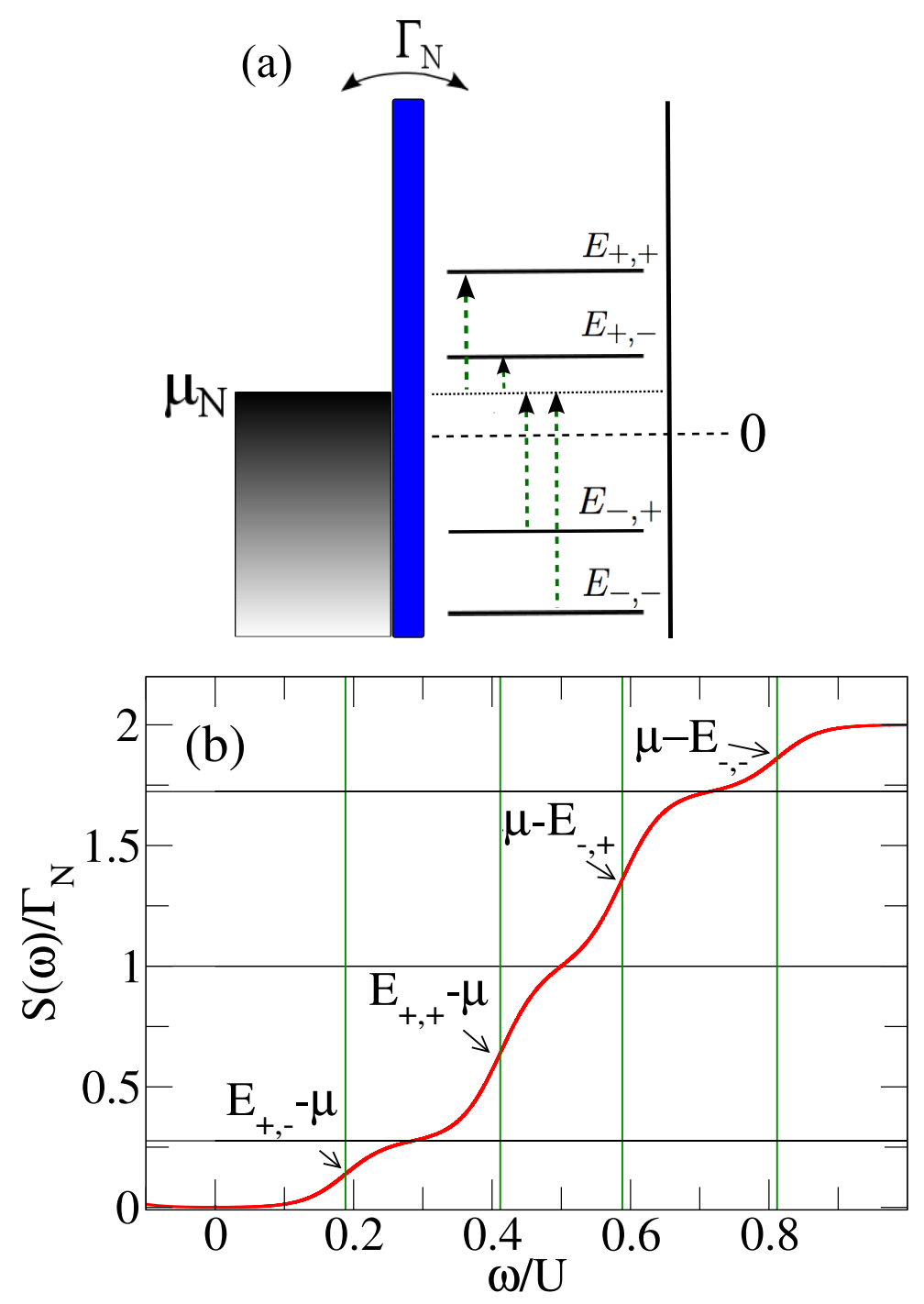

Figure 4.13: (a) Sketch of the energy landscape of the proximized single-level quantum dot for $\mu_{\mathrm{N}}=0.2 U$ and $\delta=0.1 U$. (b) Finite-frequency noise $S(\omega)$ with $\Gamma_{\mathrm{N}}=0.0002 U, \Gamma_{\mathrm{S}}=0.2 U, k_{\mathrm{B}} T=0.03 U$. 
the singly-occupied state $|\sigma\rangle$ into the ABS $|-\rangle$, the dot is singly occupied and the system is hence still in the region where the current is suppressed (see Figs. 4.12 (a) and 4.13 (a) for the energy landscape of the system and Fig. 4.9 for the respective behaviour of the current).

In this bias regime the noise spectrum exhibits four steps at noise frequencies $\omega=\left|E_{ \pm, \pm}-\mu_{\mathrm{N}}\right|$ as shown in Figs. 4.12 (b) and 4.13 (b). The reason for this is that a finite transport voltage breaks the degeneracy between the excitation energies that is present for $\mu_{\mathrm{N}}=0$. This is indicated by the different lengths of the green dashed arrows in the energy-landscape sketches of the system. The height of each of the four steps is given by the respective effective coupling strength, Eq. (3.7) and 3.8.

In the limit of zero detuning, $\delta=0$, the effective coupling to all four levels is equal, $\Gamma_{\sigma \rightarrow+}=\Gamma_{+\rightarrow \sigma}=\Gamma_{\sigma \rightarrow-}=\Gamma_{-\rightarrow \sigma}=\Gamma_{\mathrm{N}} / 2$. The step heights shown in the noise spectrum in Fig. 4.12 (b) are therefore all equal to $\Gamma_{\mathrm{N}} / 2$.

In contrast, if the detuning $\delta$ is finite, the effective coupling strengths differ. For $\delta>0$, the coupling for the excitation to go from the singlyoccupied state $|\sigma\rangle$ to the $|+\rangle$ state and to excite from the $|-\rangle$ state into the singly-occupied state $|\sigma\rangle$ is stronger than for the other two excitations, $\Gamma_{\sigma \rightarrow+}=\Gamma_{-\rightarrow \sigma}>\Gamma_{\sigma \rightarrow-}=\Gamma_{+\rightarrow \sigma}$. Consequently these first excitations give a larger contribution to the noise than the latter ones and the noise spectrum exhibits steps with different heights, see Fig. 4.13 (b).

This behavior holds only as long as the detuning does not become much larger than the coupling strength to the superconducting lead. As soon as $\delta \gg \Gamma_{\mathrm{S}}$, the noise spectrum exhibits again only two steps because the superconducting correlations on the quantum dot vanish. The effective coupling strengths of the Andreev levels corresponding to the excitation from $|\sigma\rangle$ to $|-\rangle$ and from $|+\rangle$ to $|\sigma\rangle$ go to zero, $\Gamma_{\sigma \rightarrow-}, \Gamma_{+\rightarrow \sigma} \rightarrow 0$, while the other two excitations are coupled with the effective tunnel-coupling strength $\Gamma_{\mathrm{N}}$ for $\delta \gg \Gamma_{\mathrm{S}}$, see Eq. (3.7) and (3.8). When setting the bias voltage to 0 , we find the previous result, as shown in Fig. 4.11 (b). This is due to the fact that the coupling strength is here twice as large as in the case of zero detuning, 
however only half of the excitations contribute to the current and to the noise when $\delta \gg \Gamma_{\mathrm{S}}$.

The finite-frequency noise spectrum in this low-bias regime provides a spectroscopy of the Andreev levels as well as the effective coupling strengths.

Note, that in this case, namely when the Andreev levels are outside the bias window, the noise steps always lead to an increase of the noise, regardless of whether the noise step is related to a tunnel process between the reservoir and a strongly or a weakly coupled dot resonance. The reason for this is that we here observe features at noise frequencies, which always correspond to energies necessary to excite otherwise blocked transport channels between dot resonance and reservoir. This is different if some of the Andreev levels are in the bias window, as we will discuss in the next section.

\subsubsection{Finite-bias regime}

We consider in this section two different situations for a finite transport voltage applied to the normal-conducting lead: first, the voltage is applied such, that all Andreev energies are in the bias window (high-bias regime) and second, such that part of the excitation energies lie outside the transport window (intermediate-bias regime). This allows us to study the full noise spectrum with its different contributions, similar to what we observed separately in the previous sections, Sec. 4.4.1 and Sec. 4.4.2.

The finite-frequency noise spectrum will be shown to provide a full spectroscopy of the system.

\section{High-bias regime, $\mu_{\mathbf{N}}>E_{+,+}$}

We now assume the dot to be in a regime where the bias, $\mu_{\mathrm{N}}=1.5 \mathrm{U}$, is chosen such that all Andreev energies are in the bias window, see the sketches in Fig. 4.14. Fig. 4.15 and Fig. 4.16 (a). We consider the case where the superconducting correlations on the dot are strong, which is realized for the detuning being smaller than the coupling to the superconductor, $\delta<\Gamma_{\mathrm{S}}$. 

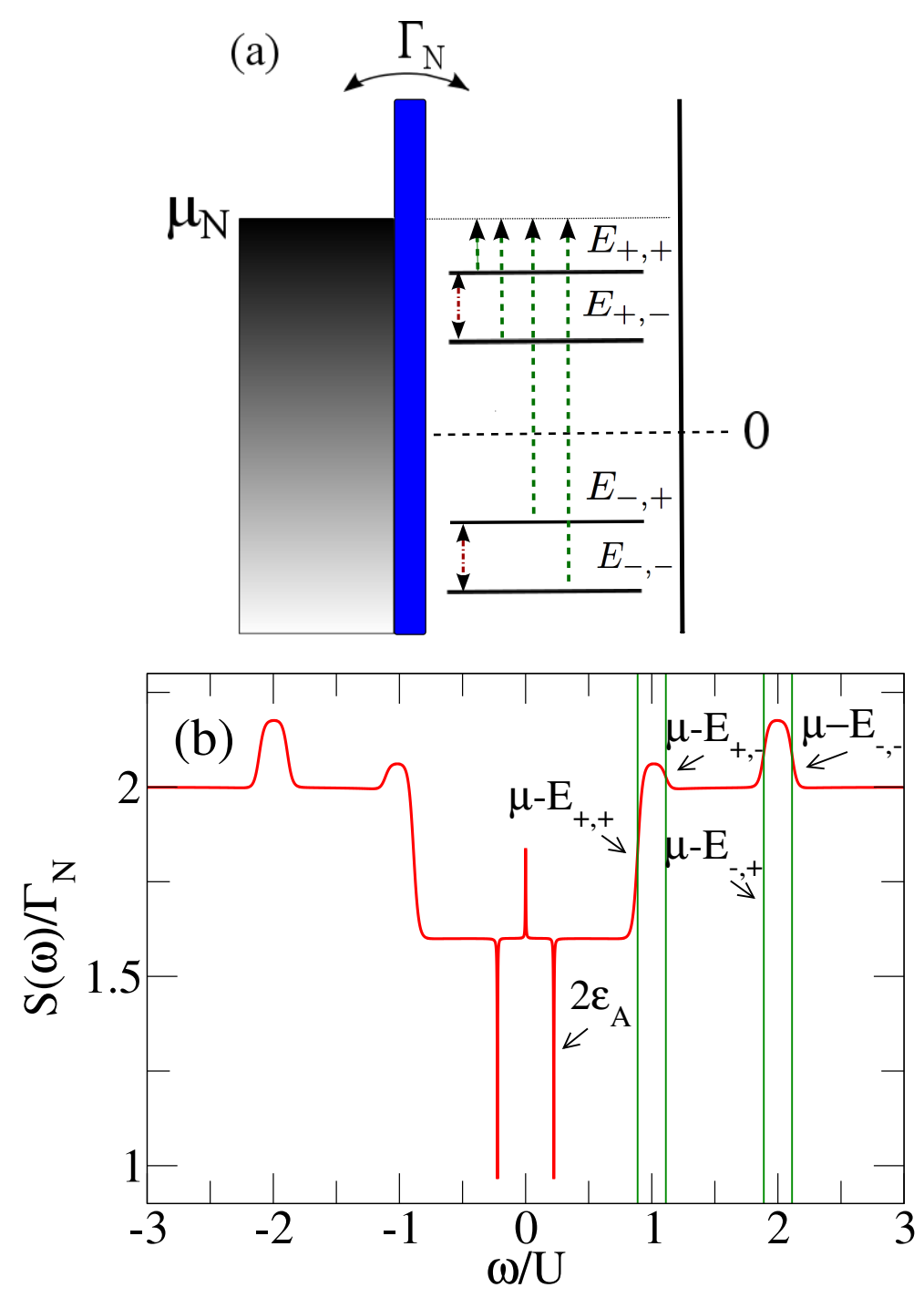

Figure 4.14: (a) Sketch of the energy landscape of the proximized single-level quantum dot for $\mu_{\mathrm{N}}=1.5 U$ and $\delta=0.1 U$. (b) Finite-frequency noise $S(\omega)$ with $\mu_{\mathrm{N}}=1.5 U, \Gamma_{\mathrm{N}}=0.002 U, \Gamma_{\mathrm{S}}=0.2 U, k_{\mathrm{B}} T=0.02 U$. 
(a)
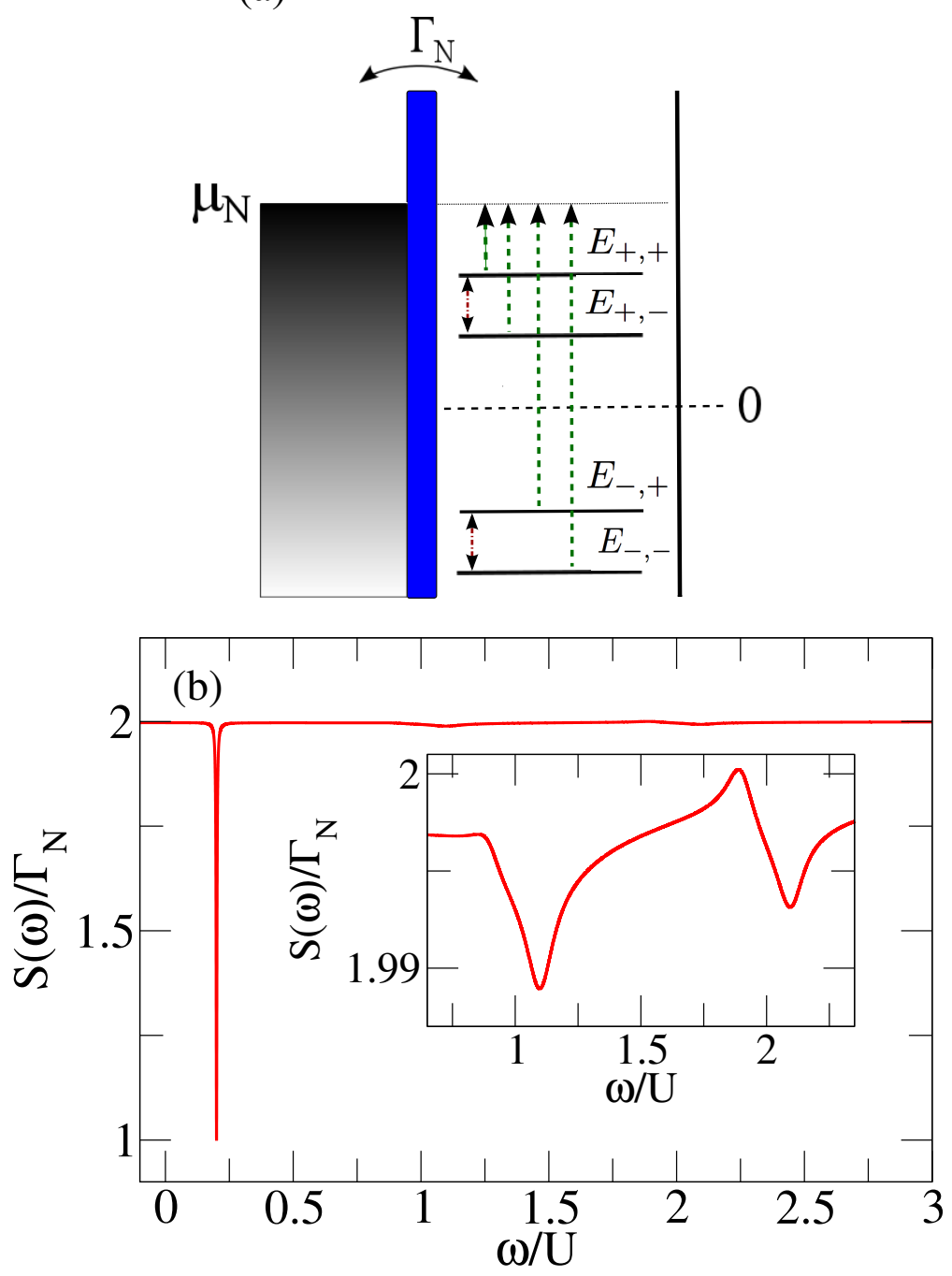

Figure 4.15: (a) Sketch of the energy landscape of the proximized single-level quantum dot for $\mu_{\mathrm{N}}=1.5 U$ and $\delta=0$. Finite-frequency noise $S(\omega)$ with $\mu_{\mathrm{N}}=1.5 U, \Gamma_{\mathrm{N}}=0.002 U, \Gamma_{\mathrm{S}}=0.2 U, k_{\mathrm{B}} T=0.02 U$. 

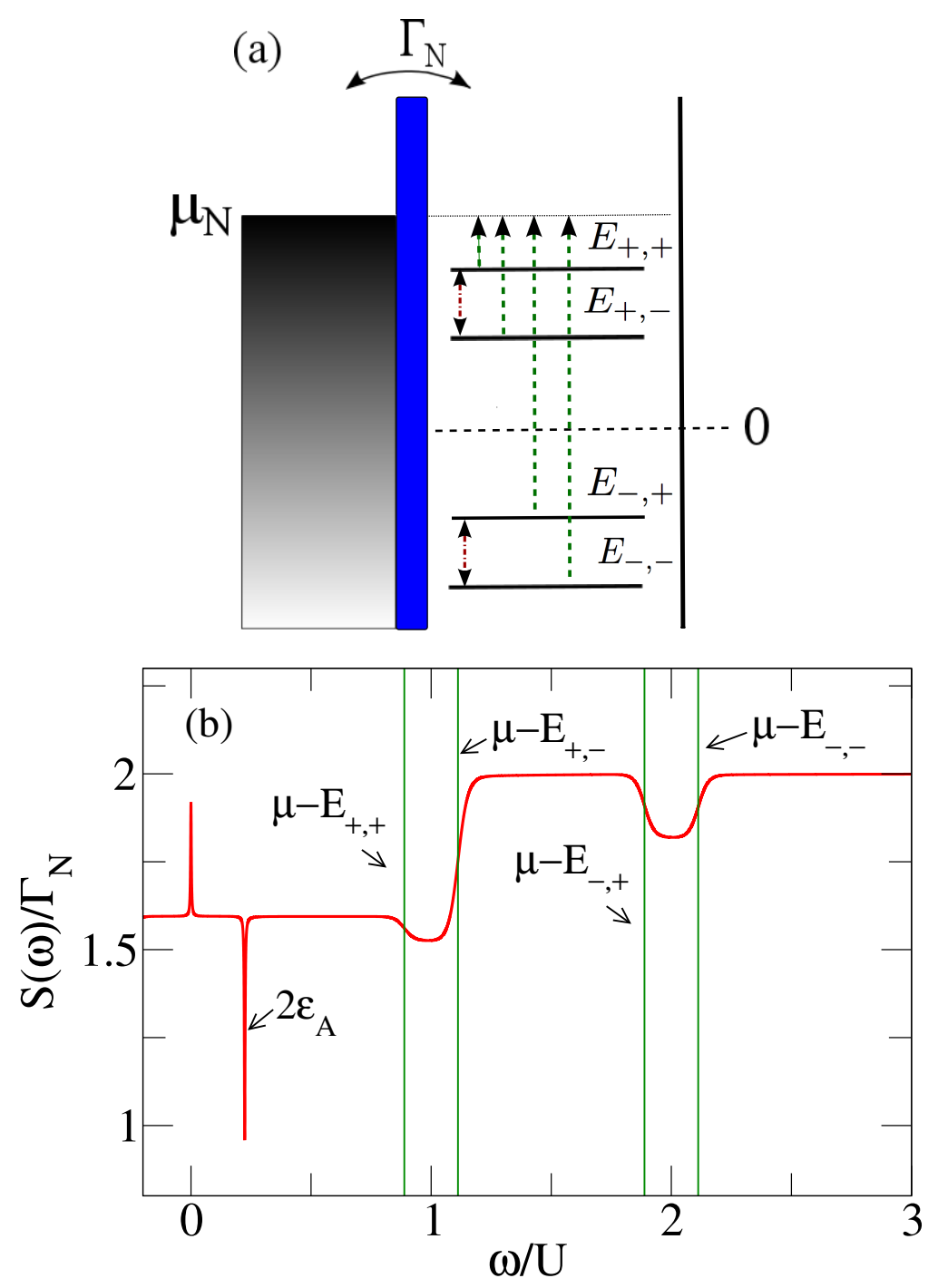

Figure 4.16: (a) Sketch of the energy landscape of the proximized single-level quantum dot for $\mu_{\mathrm{N}}=1.5 U$ and $\delta=-0.1 U$. (b) Finite-frequency noise $S(\omega)$ with $\mu_{\mathrm{N}}=1.5 U, \Gamma_{\mathrm{N}}=0.002 U, \Gamma_{\mathrm{S}}=0.2 U, k_{\mathrm{B}} T=0.02 U$. 
The results for the noise spectrum in this regime are shown in Fig. 4.14 for $\delta=0.1 U, \delta=0$ in Fig. 4.15 , or $\delta=-0.1 U$ Fig. 4.16. In this regime the Andreev current is close to maximal, corresponding to the upper edge of the density plot in Fig. 4.9.

In the low- and intermediate-frequency regimes, $\omega<\left|E_{+,+}-\mu_{\mathrm{N}}\right|$, the noise spectrum shows Lorentzian-shaped features, as discussed in Sec. 4.4.1. These are a peak for a non-zero detuning $\delta$ in the low-frequency regime, $\omega<\Gamma_{\mathrm{N}}$, and dips at a noise frequency equal to the splitting of the ABSs, $\omega=\left|\epsilon_{+}-\epsilon_{-}\right|=2 \epsilon_{\mathrm{A}}$, due to a coherent destructive interference of the ABSs.

The high-frequency part of the noise spectrum of Fig. 4.14 and Fig. 4.16 exhibits quantum noise steps at frequencies $\omega=\left|E_{ \pm, \pm}-\mu_{\mathrm{N}}\right|$, similar to what was discussed in Sec. 4.4.2. However, in contrast to the previous section, where all steps lead to an increase of the noise, the quantum noise steps found here show different signs depending on the effective coupling strength. A noise process related to a strongly coupled Andreev level leads to an increase of the noise, while a process between the electronic reservoir and a weaker coupled Andreev level decreases the noise. The steps occurring in the finite-bias regime at high frequencies can be understood from an analogy to the ones obtained for a quantum dot coupled to normal-conducting leads. In this case an asymmetric coupling to the two normal-conducting leads takes the role of the differently coupled Andreev levels in the hybridised dot. See the previous section 4.1.1 for a detailed discussion of this simpler case.

From this we deduce that the varying directions of the noise steps originate from the competition between different noise contributions of opposite sign. When a certain noise frequency $\omega$ is reached, an ensemble of transport processes becomes visible which can in principle involve different types of transitions, $|\sigma\rangle \leftrightarrow| \pm\rangle$, due to tunneling with the normal lead. The contribution to the noise which stems from correlations of tunnelling processes involving only one type of transitions tends to increase the noise, while the noise contribution stemming from correlations of tunnelling processes with different transitions tends to decrease the noise. Depending on which of these 
contributions has the larger magnitude, the step is positive or negative. The magnitude of the correlations in turn depends on the coupling strength of the involved processes. The addition of these two noise contributions hence yields the noise spectra shown in Fig. 4.14, Fig. 4.15 and Fig. 4.16, In the following we discuss the implications of this effect for different magnitudes of the detuning $\delta$.

In Fig. 4.14 (b), we observe a large increase of the noise at the noise frequency $\omega=\left|\mu_{\mathrm{N}}-E_{+,+}\right|$, because the related Andreev level is coupled strongly to the normal-conducting lead. This noise frequency corresponds to the energy which an electron on the dot needs to absorb in order to tunnel out of the dot. The second step at $\omega=\left|\mu_{\mathrm{N}}-E_{+,-}\right|$occurs when the the noise frequency provides the energy for an electron to tunnel out of the weaker coupled Andreev level $E_{+,-}$. The noise step stemming from the process with the weaker coupled Andreev level has a negative sign. In Fig. 4.14 (b) the noise increases again at $\omega=\left|\mu_{\mathrm{N}}-E_{-,+}\right|$and decreases at $\omega=\left|\mu_{\mathrm{N}}-E_{-,-}\right|$. For even higher frequencies, $\omega \gg \mu_{\mathrm{N}}$, the noise is always given by the sum of the effective coupling strengths, $2 \Gamma_{\mathrm{N}}$, since the noise frequency provides enough energy to excite from the singly-occupied state into either one of the ABSs and vice versa.

If we invert the order of the excitation energies or the strength of their effective couplings, the broad maxima are transformed into troughs. This can for example be achieved by inverting the bias $\mu_{\mathrm{N}} \rightarrow-\mu_{\mathrm{N}}$ or the detuning $\delta \rightarrow-\delta$. Figure 4.16 (a) shows the energy landscape and (b) the finitefrequency noise spectrum for $\delta=-0.1 U$. The detuning is reversed compared to the previously discussed case in Fig. 4.14 (b). Consequently, the noise is first suppressed, when a process with a weakly coupled Andreev level takes place, and then enhanced to the value $2 \Gamma_{\mathrm{N}}$ at a noise frequency related to a strongly coupled Andreev level, see Fig. 4.16 (b).

The quantum steps in the noise spectrum occur only in a regime of intermediate detuning $\delta$. If the detuning $\delta$ becomes larger than the coupling to the superconducting lead $\Gamma_{\mathrm{S}}$, the step structure as shown in Fig. 4.14 and 
Fig. 4.16 gets suppressed, because the superconducting correlations become weaker.

If the proximity effect is on resonance and the detuning is exactly equal to zero $(\delta=0)$ the effective coupling strengths to the different Andreev levels are equal. The finite-frequency noise spectrum for this case is displayed in Fig. 4.15 (b) with the corresponding energy landscape of the proximized dot, Fig. 4.15 (a). In this regime, when the probabilities of the dot to be in one of the ABSs are equal, no steps but only shallow dips appear in the spectrum due to an almost complete compensation of the different noise contributions. The high-frequency noise spectrum is given by the sum of the effective coupling strengths, $2 \Gamma_{N}$. Only small features at $\omega=\left|\mu-E_{ \pm, \pm}\right|$ remain as shown in the inset of Fig. 4.15 (b).

Intermediate-bias regime, $E_{+,-}<\mu_{\mathbf{N}}<E_{+,+}$

We finally also address the case of the intermediate bias regime, where only a part of the levels are in the bias window. In Fig. 4.17 (a), we show the energy landscape of the dot considered here, with $\mu_{\mathrm{N}}=0.55 \mathrm{U}$ and $\delta=$ $0.1 U$, where the excitation energy $E_{+,+}$is outside the bias window. The noise spectrum of the intermediate-bias regime, see Fig. 4.17 (b), shows a mixture of the previously observed effects in the unidirectional, low-bias and high-bias regime. We can identify in the spectrum the features discussed in Sec. 4.4.1, namely the Lorentzian dependence in the low-frequency regime and the resonance dips at $\omega=2 \epsilon_{\mathrm{A}}$, which are a signature of the coherent transfer of Cooper pairs between dot and superconducting lead.

Furthermore, the quantum noise steps show an overlap of the features discussed in the previous subsections: the steps at $\omega=\left|E_{+,+}-\mu_{\mathrm{N}}\right|$ and $\omega=\left|\mu_{\mathrm{N}}-E_{-,-}\right|$both lead to an increase of the noise but with a different step height due to the fact that the corresponding Andreev levels couple with different effective coupling strengths to the reservoir. The first of these steps overlaps with the intermediate-frequency regime, namely where the resonance dips due to the internal dynamics occur. The spectrum furthermore shows 
two steps at frequencies $\omega=\left|\mu_{\mathrm{N}}-E_{-,+}\right|$and $\omega=\left|\mu_{\mathrm{N}}-E_{+,-}\right|$, where the direction of the steps tells us if it is a noise process between the normal conducting lead and a strongly or weakly coupled Andreev level. Note that also the resulting trough is here partly found in the intermediate-frequency regime.

At even higher frequencies, $\omega \gg \mu_{N}$, the noise is again given by the sum of the effective coupling strengths.

\subsection{Conclusions}

In this Chapter we have presented results for the finite-frequency noise spectrum of a system composed of a single-level quantum dot. In the first section we have calculated the finite-frequency noise through a single-level quantum dot weakly coupled to normal conducting leads based on the diagrammatic real-time approach for noise introduced in 3.4. The noise spectrum of this system has been studied previously in Refs. $29,33,36,37,40,43,45$ using various methods. We discuss the symmetrised current noise for different bias regimes for the non-interacting quantum dot and also with finite Coulomb repulsion on the quantum dot. We find that in the quantum noise regime, when $\omega>k_{\mathrm{B}} T, V$, the noise spectrum shows steps at $\omega=\left|\mu_{\mathrm{R} / \mathrm{L}}-\epsilon\right|$, whose height can be understood in terms of the coupling strength to the leads. If the energy level lies within the bias window the steps in the noise spectrum can have different sign depending if the dot is symmetrically or asymmetrically coupled. Whether a step in the noise spectrum leads to an increase or a decrease of the total noise depends on the coupling strength of the different excitations and the noise stemming from correlations in the same lead increases at frequencies equal to the excitation energies, while the noise due to correlations between different leads decreases.

In the second section 4.2 we consider a single-level quantum dot coupled to a superconductor and a normal-conducting lead. The noise spectrum reflects the internal spectrum of the proximized dot. In particular, we found 

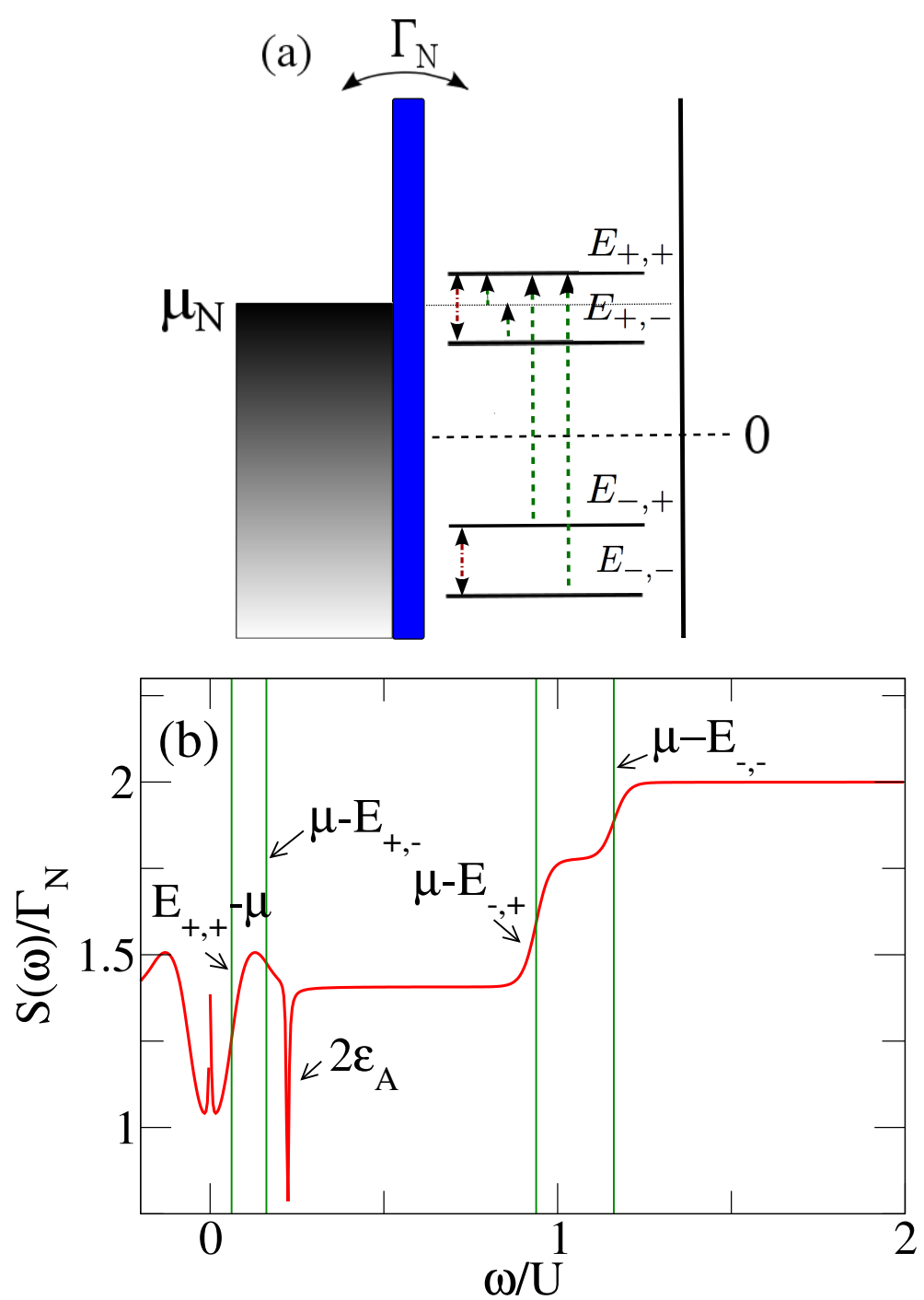

Figure 4.17: (a) Sketch of the proximized single-level quantum dot for $\mu_{\mathrm{N}}=$ $0.55 U$ and $\delta=0.1 U$. (b) Finite-frequency noise $S(\omega)$ with $\Gamma_{\mathrm{N}}=0.002 U$, $\Gamma_{\mathrm{S}}=0.2 U, k_{\mathrm{B}} T=0.02 U$. 
resonance dips occur at a frequency equal to the splitting of the ABSs, $\omega=$ $2 \epsilon_{\mathrm{A}}$. This feature is a signature of the coherent oscillation of Cooper pairs between quantum dot and superconductor. The effect is strongest if the superconducting correlations on the dot are maximal, which happens when the proximity effect is on resonance, $\delta=0$.

The high-frequency regime of the noise spectrum shows quantum-noise steps at frequencies $\omega=\left|E_{ \pm, \pm}-\mu_{\mathrm{N}}\right|$. The quantum-noise steps provide not only information on the Andreev addition energies of the system, but also on the effective coupling strength of the Andreev levels to the normal conducting lead. The height (in the low bias regime) and sign (in the finite-bias regime) of the steps tell the strength of the effective coupling of each Andreev level to the reservoir. We found that the steps appearing in the noise spectrum can be understood from an analogy to the ones obtained for a quantum dot coupled to normal-conducting leads, Sec. 4.1. In this case an asymmetric coupling to the two normal-conducting leads takes the role of the differently coupled Andreev levels in the hybridized dot.

Therefore, we conclude that the finite-frequency noise spectrum provides a full spectroscopy of the proximized quantum dot.

In order to observe the features of the spectrum experimentally, the measurement of the finite-frequency noise has to be performed in the $\mathrm{GHz}$ regime. For example, in recent experimental realization of such a hybrid-quantum dot device [14, 138] the coupling to the superconductor is $\Gamma_{\mathrm{S}} \approx 50-250 \mathrm{GHz}$. 


\section{Chapter 5}

\section{Finite-time full counting statistics}

In Chapter 4 we have presented results for the finite-frequency noise in transport through a quantum dot and demonstrated that the current fluctuations contain information on the transport properties and the internal time scales of the system which cannot be extracted from the average current. However, there might be even more information contained in the current fluctuations which are not visible in the noise spectrum. A complete statistical description of the transport processes are captured by the FCS [26, 27]. The probability distribution provides a full statistical characterization of the tunneling events through the quantum dot. This allows us to calculate moments and cumulants of the current fluctuations of arbitrary order. In the last decade it has become feasible to measure the higher order cumulants and also the entire distribution function of tunneling events $25,28,58,59,61,105,114$. Hence, FCS is nowadays an established concept not only in theoretical but also in experimental physics.

In this chapter we present results for finite-time FCS for subgap transport through a single-level quantum dot tunnel-coupled to a normal and a superconducting lead based on the theory introduced in Sec. 3.5. We consider strong coupling to the superconductor while the normal lead is only 
coupled weakly to the quantum dot. The model of the system has been introduced in Sec. 3.1 and the reduced density matrix in Sec. 4.2. Most studies about FCS consider the long-time limit, i.e correlation effects are integrated over a long period of time and important information about characteristic timescales are not visible. The FCS in the long-time limit for a quantum dot coupled to a normal and a superconducting lead has been studied previously in the large gap limit 154 and for a finite superconducting gap 47]. Here, we focus on the finite-time FCS to reveal the internal dynamics of the system on the short-time scale. Based on a non-Markovian master equation (NMME) we determine the generalized Fano factor as a function of time for current cumulant up to order $k=5$.

We discuss in Sec. 5.1 the unidirectional transport regime. In a first step we recover the FCS for the system in the long-time limit 5.1.1 while we also consider the coherences, i.e. the coherent superposition between the doubly occupied and empty state. In Sec. 5.2 we summarize and conclude the results of this chapter.

\subsection{Unidirectional transport regime}

We start with presenting results in the unidirectional transport regime, where the applied bias voltage is larger than all other energy scales except the superconducting gap $\Delta$, ensuring that the electron transport is unidirectional and all Andreev levels are within the bias window $\left(\mu \gg E_{+,+}\right)$. In the unidirectional transport regime the NMME Eq. (3.43) simplifies to the Markovian limit. Since we consider only the first order in the tunnel coupling strength, the inhomogeneous term vanishes, $\boldsymbol{\Gamma}(\chi, z)=0$ and the self-energy kernel $\boldsymbol{W}$ becomes independent of frequency $z$. 


\subsubsection{Long-time limit}

In a first step, we review the FCS in the long-time limit $(t \rightarrow \infty)$ by also considering the coherences in the reduced density matrix. In the long-time limit we have to consider only the zero-frequency limit of the Laplace transform of the self-energy kernel $\boldsymbol{W}(\chi)$. The long-time behavior can be determined by the eigenvalue of the kernel $\boldsymbol{W}(\chi)$ with the smallest absolute value of the real part [103, 157]. In the long-time limit the CGF is given by

$$
S\left(t, \chi_{\mathrm{N}}\right)=t\left(-\Gamma_{N}+\frac{\sqrt{\Gamma_{N}^{2}-4 \epsilon_{A}^{2}+\sqrt{4\left(e^{-2 i \chi_{\mathrm{N}}}-1\right) \Gamma_{N}^{2} \Gamma_{N}^{2}+\left(\Gamma_{N}^{2}+4 \epsilon_{A}^{2}\right)^{2}}}}{\sqrt{2}}\right)
$$

which simplifies for $\Gamma_{\mathrm{N}} \ll \Gamma_{\mathrm{S}}$ to the CGF represented in Ref. [154]. The counting field of the normal conducting lead is represented by $\chi_{\mathrm{N}}$. For the system under consideration the total current is approximately given by the particle current coming from the normal conducting lead, since we consider a strong coupling to the superconductor, $\Gamma_{\mathrm{S}} \gg \Gamma_{\mathrm{N}}$. This has been discussed in the context of displacement currents in Sec. 3.3 .

Throughout this section we study the generalized Fano factor $F^{k}$, which has been defined in Sec. 3.5 as $F^{k}=\left\langle\kappa^{k}(t)\right\rangle /\left\langle\kappa^{1}(t)\right\rangle$, where $\kappa^{k}(t)$ is the $k$ th order cumulant.

Figure 5.1 shows the generalized Fano factor in the long-time limit as a function of the detuning $\delta$ up to an order of $k=7$. In the long-time limit the generalized Fano factor is independent of the time t. This is obvious, since the CGF, Eq. 5.1, is linear in time $t$. The generalized Fano factor Fig. 5.1 shows a transition from $F^{k}=1$ at $\delta=0$, where the proximity effect is on resonance and the superconducting correlation on the dot is maximal, to $F^{k} \approx 2^{k-1}$ for a large detuning $\delta\left(\delta \gg \Gamma_{S}\right)$. In the latter case the proximity effect is off-resonance and the BCS-like correlation on the dot is almost zero, as discussed in Ref. 154.

As depicted in Fig. 5.1 starting from order $k=5$ the transition from a Fano factor 1 to $2^{k-1}$ is not monotonic anymore and the generalized Fano 
factor starts to oscillate as a function of the detuning $\delta$. The generalized Fano factor grows factorially in magnitude with order $k$ as well as its oscillation. This behavior is universal and independent of the system [60,61].

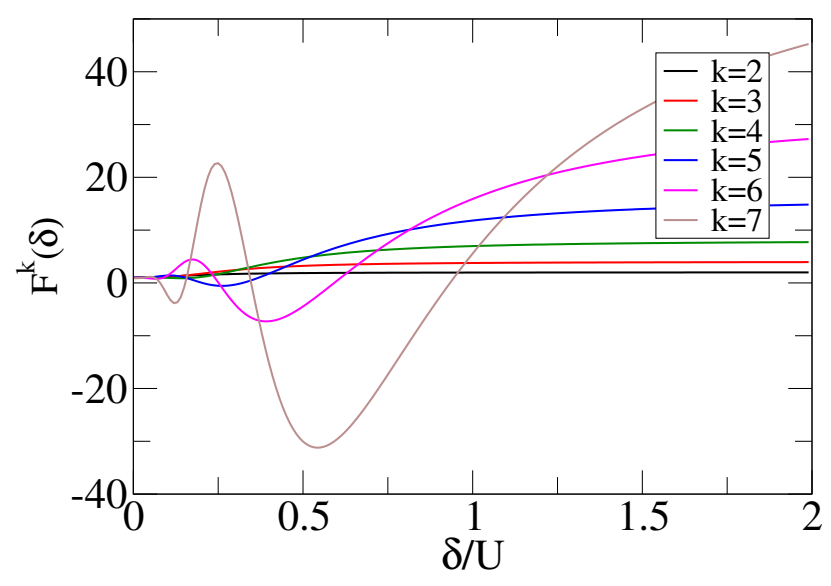

Figure 5.1: Generalized Fano factor $F^{k}$ as a function of $\delta$ in the long-time limit. The other parameters are $\Gamma_{\mathrm{S}}=0.2 U$ and $\Gamma_{\mathrm{N}}=0.002 U$.

\subsubsection{Finite-time regime}

Now, we turn our attention to the the main purpose of the present chapter, namely the finite-time FCS. The theory has been introduced in Sec. 3.5. The central object of the theory is the MGF $G(\chi, z)$ which is given by the trace over the reduced density matrix of the system. The first derivative and the second derivative of the MGF gives direct access to the current and the finite-frequency noise. Both the current and the finite-frequency noise of a quantum dot coupled to a normal and a superconducting lead have been discussed in the previous Chapter 4 and are not the scope of the present discussion. Here, we like to present results for the FCS in terms of the Fano factor $F^{k}$ for orders $k \geq 2$. In particular, we are interested in the short-time regime of higher orders and the generalized Fano factor as a function of real time $t$. 

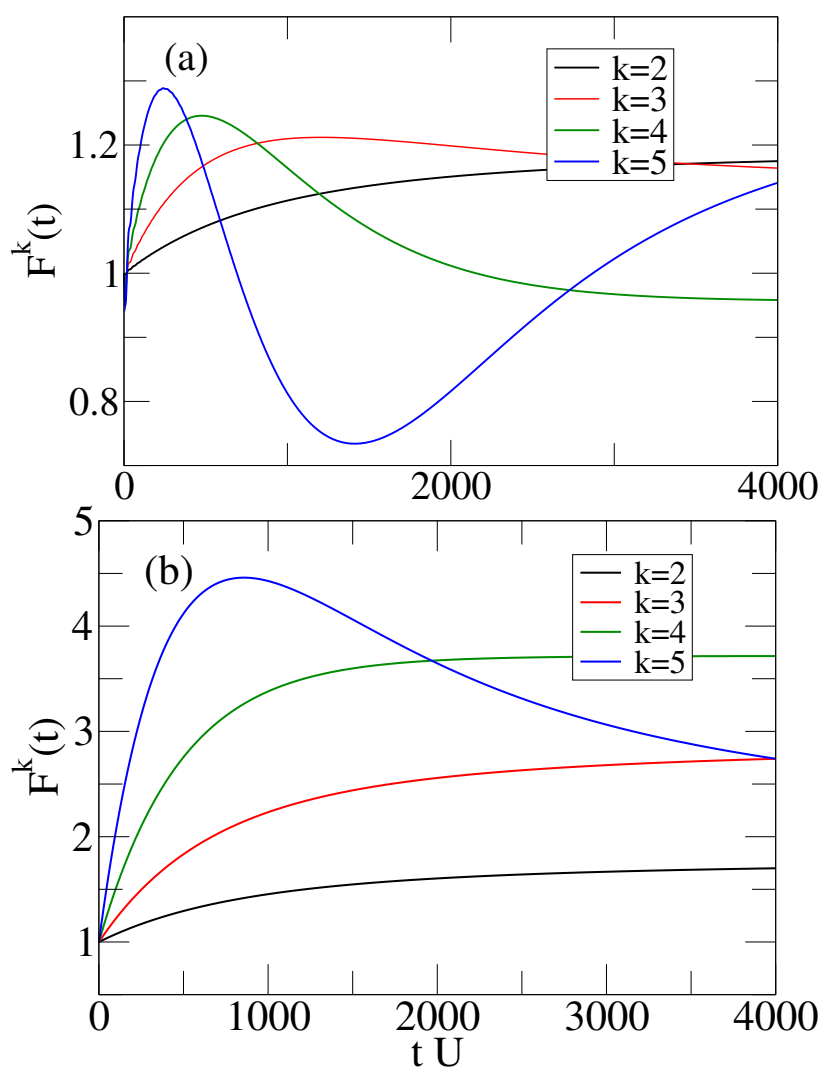

Figure 5.2: Generalized Fano factor $F^{k}$ in the unidirectional transport regime as a function of time $t$. The other parameters are $\Gamma_{\mathrm{S}}=0.2 U, \Gamma_{\mathrm{N}}=$ $0.002 U$ and (a) $\delta=0.1 U$ and (b) $\delta=0.4 U$. 
Figure 5.2 shows the generalized Fano factor as a function of time $t$ up to an order $k=5$ for (a) a detuning of $\delta=\Gamma_{\mathrm{S}} / 2$ and (b) $\delta=2 \Gamma_{\mathrm{S}}$. In the first case $\delta=\Gamma_{\mathrm{S}} / 2$ (Fig. 5.2 (a)) the proximity effect is on resonance, i.e the Andreev current is in the region where its largest. Remember, the Andreev current is maximal if the detuning is minimal, $\delta \approx 0$. The generalized Fano factor as displayed in Fig. 5.2 (a) shows a monotonic transition up to an order $k=3$ from 1 at $t=0$ to the long-time limit. Starting from order $k=5$ the generalized Fano factor has a minimum for short times and starts to oscillate.

Figure 5.2 (b) shows the generalized Fano factor as a function of $t$, when the proximity effect is off-resonance $\delta \gg \Gamma_{\mathrm{S}}$, namely the regime where the Andreev current is suppressed and BCS-like correlations on the quantum dot are almost zero. Again, we show the generalized Fano factor up to an order of $k=5$. We observe that the generalized Fano factor retains the monotonic transition to the long-time limit to higher orders compared to Fig. 5.2 (a) and hence, the oscillatory behavior starts for higher orders. Additionally, the Fano factor grows to higher values with increasing order compared to Fig. 5.2 (a). The increase of the magnitude of the generalized Fano factor with increasing detuning $\delta$ is in agreement with the result for the long-time limit, Fig. 5.1.

In general Fig. 5.2 as well as Fig. 5.1 for the long-time limit show a generic behavior of higher order cumulants for transport through a Coulomb blockade nanostructure such as a quantum dot. Both show that the higher order cumulants grow factorially in magnitude with cumulant order and oscillate as a function of essentially any parameter. This oscillatory behavior of the higher order cumulants is universal for any system [61, 158] and has been shown experimentally as well [61, 113]. The universality of the oscillations stems from general mathematical properties of the CGF and higher-order derivatives [159]. Even though the results for the generalized Fano Factor $F^{k}(t)$ as a function of time show a generic behavior, we find a difference depending on the detuning $\delta$ or the strength of the superconducting correlation 
on the quantum dot, respectively.

\section{Short-time behavior}

We now focus on the short-time range of the generalized Fano factor. We find that at the short-time scale the Fano factor $F^{k}(t)$ oscillates as shown in Fig. 5.3. The oscillation depends on the strength of the detuning $\delta$. The oscillatory behavior becomes most prominent when the proximity effect is on resonance $\delta=0$ (see Fig. 5.3 (a)). On resonance the superconducting correlations on the dot are maximal and Cooper pairs oscillate rapidly between the quantum dot and the superconductor. With increasing detuning $\delta$ the oscillation gets suppressed Fig. 5.3 (c) and vanishes when the detuning $\delta$ becomes larger than the coupling to the superconductor $\Gamma_{\mathrm{S}}$, see Fig. 5.3 (c). The reason for this is that for $\delta \gg \Gamma_{\mathrm{S}}$ the superconducting correlations on the dot are almost zero and the resulting short time behavior is similar to the case of a quantum dot with normal-conducting leads only. The oscillation frequency is given by $2 \epsilon_{A}$, the splitting between the ABS. The visibility of the oscillation increases with order $k$.

The oscillation frequency $\omega=2 \epsilon_{A}$ is equal the oscillation frequency of Cooper pairs which tunnel back and forth between dot and superconductor. The oscillatory behavior at short times is a signature of the coherent transfer of Cooper pairs between superconducting lead and quantum dot. This information on the coherent dynamics of the system and its characteristic time scale can be found also in the finite-frequency noise spectrum as discussed in Chapter 4, where it appears as a noise-suppressing resonance dip at frequency $\omega=2 \epsilon_{A}$.

Furthermore, these coherent dynamics underlying the proximity effect in the dot show also up in the waiting time distribution of transport events in the normal lead, which exhibits an oscillatory behavior [155] similar to the short-time behavior found here. 

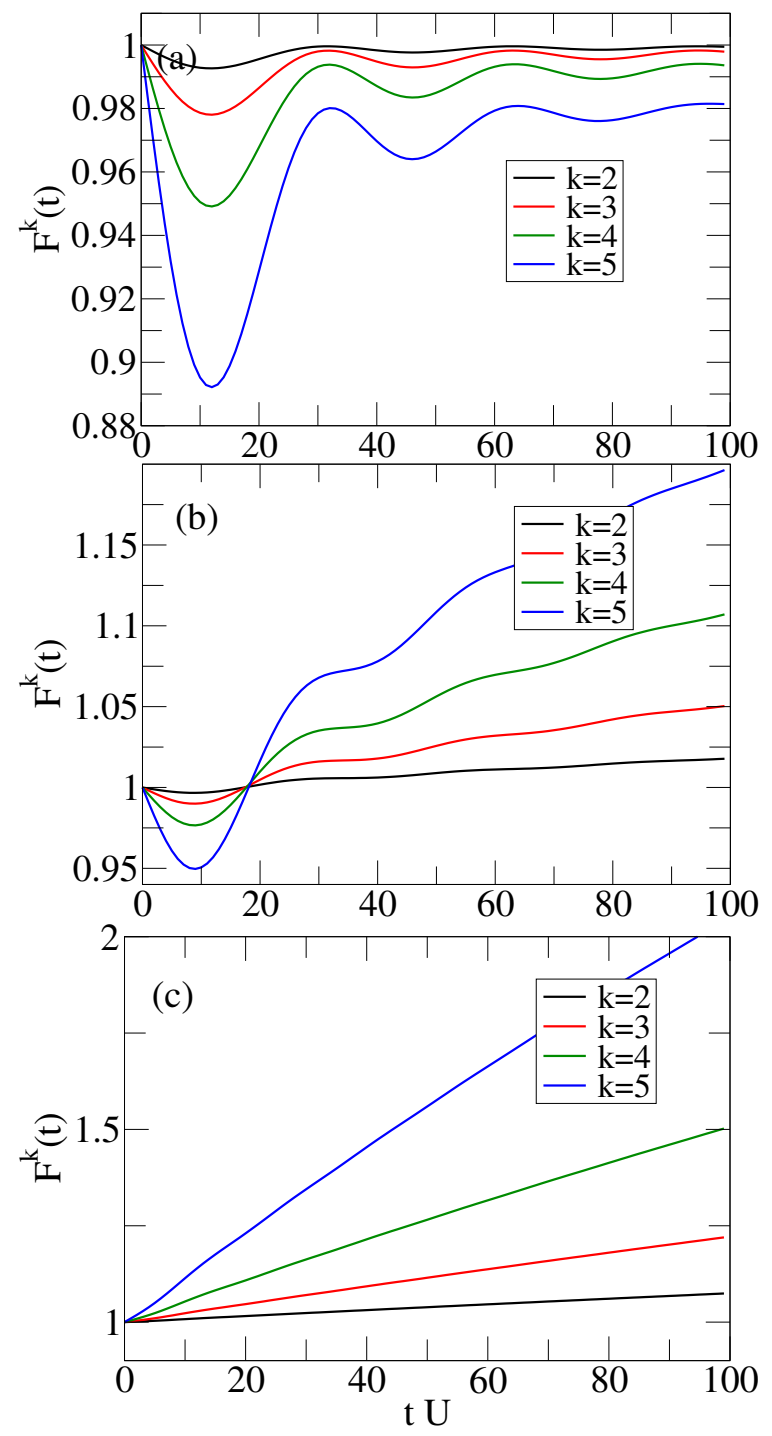

Figure 5.3: Generalized Fano factor $F^{k}$ in the unidirectional transport regime as a function of time $t$. The other parameters are $\Gamma_{S}=0.2 U, \Gamma_{N}=0.002 U$ and (a) $\delta=0 U$, (b) $\delta=0.1 U$ and (c) $\delta=0.4 U$. 


\subsection{Conclusions}

In this chapter we have presented a calculation of the finite-time FCS based on the theory presented in Sec. 3.5 of a system composed by a single-level quantum dot tunnel-coupled to a superconductor and a normal-conducting lead. We found that the FCS reflects the coherent dynamics underlying the proximity effect. For short times, the generalized Fano factor oscillates with a frequency equal to the splitting of the ABS, $\omega=2 \epsilon_{A}$. This short time behavior is a signature of the coherent transfer of Cooper pairs between quantum dot and superconductor. The magnitude of the oscillation increases with increasing cumulant order $k$. The oscillatory behavior vanishes for a large detuning $\delta \gg \Gamma_{\mathrm{S}}$, when the superconducting correlations on the quantum dot is almost zero.

Beyond the short-time regime the generalized Fano factor grows in magnitude and starts to oscillate with increasing cumulant order. These oscillations occur as function of essentially any parameter of the system. This behavior of the higher order cumulants is universal for any system [61, 158] and has been shown experimentally as well [61,113].

The results of this chapter consider the unidirectional transport regime only. Hence, the presented results are Markovian, since we consider a weakcoupling to the normal-conducting lead. However the theory set up in Sec. 3.5 allows also the inclusion of non-Markovian dynamics. A complete study of the system's finite-time FCS in terms of different bias regimes, which allow the inclusion of non-Markovian effects, has still to be done. 


\section{Chapter 6}

\section{Finite-frequency skewness}

In this chapter we present results for the frequency-dependent skewness for transport through a single-level quantum dot weakly coupled to a normal and strongly to a superconducting lead. The model has been introduced in Sec. 3.1. The finite-frequency skewness is defined as the third order of the power spectrum

$$
\begin{aligned}
S\left(\omega_{1}, \omega_{2}, \omega_{3}\right)= & \int_{-\infty}^{\infty} d t_{1} \int_{-\infty}^{\infty} d t_{2} \int_{-\infty}^{\infty} d t_{3} e^{-i\left(\omega_{1} t_{1}+\omega_{2} t_{2}+\omega_{3} t_{3}\right)} \times \\
& \mathcal{T}\left\langle I\left(t_{1}\right) I\left(t_{2}\right) I\left(t_{3}\right)\right\rangle
\end{aligned}
$$

where $\mathcal{T}$ is the symmetrization operator that sums over all possible time switches.

We consider the unidirectional transport regime, where the applied bias voltage is larger than all other energy scales of the system except the superconducting gap $\Delta$ to prevent that electrons can tunnel in the opposite direction of the mean current. In Chapter 4 we have derived and discussed the finite-frequency noise and demonstrated that the frequency-dependent noise spectrum gives information on characteristic time scales of the system, which are not visible in the zero-frequency limit. It has been shown theoretically 160,161 that also the frequency-dependent skewness may contain further information on the internal time scales and the correlations. 
Here, we apply the theory of multi-time FCS presented in Sec. 3.6 to derive results for the finite-frequency skewness. For a complete characterization of the transport processes of the system it is necessary to investigate the current fluctuations at all time and frequency scales.

The finite-frequency noise in the unidirectional transport regime as discussed in Sec. 4.4.1 results in a Lorentzian peak in the low-frequency regime and features a resonance dip at $\omega=2 \epsilon_{\mathrm{A}}$ for intermediate frequencies. However, the noise is a one-frequency quantity and does not reflect correlations between different spectral components of the current.

In order to study higher-order current correlations, i.e. correlations between different counting intervals (see Sec. 3.6), we have to consider the polyspectrum $S^{(k)}\left(\omega_{1}, \ldots, \omega_{k-1}\right)$, Eq. 3.61. The polyspectrum $S^{(k)}$ is defined as the $k$-time averaged current cumulants in frequency space. As presented in Sec. 3.6 the polyspectrum can be obtained from the frequency-dependent FCS. In particular, for $k=2$ we get the known finite-frequency noise, while for $k=3$ we obtain the bispectrum, namely the finite-frequency skewness. The spectral density for $k=3$ depends on two frequencies. Here, we will study the frequency-dependent skewness determined from the multi-time FCS introduced in Chapter 3. In principle, the formalism allows also to extract current cumulants of higher orders.

\subsection{Low-frequency regime}

In a first step, we derive the low-frequency contribution of the skewness $S_{\text {low }}^{(3)}(\omega, \Omega)$, where the relevant frequencies are of the order of the coupling strength to the normal-conducting lead. We obtain for $k=3$ the following Fano factor $\left(F^{(k)}=S^{(k)} / I\right)$ in the low-frequency regime,

$$
\begin{aligned}
F_{\text {low }}^{(3)}(\omega, \Omega)= & \frac{S_{\text {low }}^{(3)}(\omega, \Omega)}{I}=1+ \\
& \frac{4 \epsilon_{\mathrm{A}}^{2} \prod_{j=1}^{2} 2 \Gamma_{\mathrm{N}} \delta \epsilon_{\mathrm{A}}\left(\gamma_{j}+\omega^{2}-\omega \Omega+\Omega^{2}\right)}{\prod_{i=1}^{3} 4 \epsilon_{\mathrm{A}}^{2}\left(v_{i}^{2}+\Gamma_{\mathrm{N}}^{2}\right)}
\end{aligned}
$$


with $\gamma_{1}=3 \Gamma_{\mathrm{N}}^{2}$ and $\gamma_{2}=\Gamma_{\mathrm{N}}^{2} \delta^{2} / 4 \epsilon_{\mathrm{A}}^{2}$ as well as the frequencies $v_{1}=\omega, v_{2}=\Omega$ and $v_{3}=\omega-\Omega$. Analogously to the second order Fano factor Eq. (4.7), the expression Eq. 6.2 takes the value $F^{(3)}=1$ related to a Poissonan transfer, if the proximity effect is on-resonance, i.e the superconducting correlation on the quantum dot is maximal. In the limit $\delta \gg \Gamma_{\mathrm{S}}$, when the proximity effect is off-resonance and the superconducting correlation on the dot is almost zero, the third order Fano factor behaves similarly to the case of a singleresonant level coupled to two normal leads as presented in Ref. [58, 104]. As discussed in Sec. 4.4.1 the low-frequency noise shows a Lorentzian dependence on the frequency $\omega$ with a width given by the coupling strength to the normal conducting lead $\Gamma_{\mathrm{N}}$. However, the frequency-dependent skewness $S_{\text {low }}^{(3)}(\omega, \Omega)$ (Eq. (6.2) ) is not just a simple Lorentzian shaped function. In Fig. 6.1 and 6.2 contour plots of the third order Fano factor $F_{\text {low }}^{(3)}(\omega, \Omega)$ are displayed for different values of the detuning. Figure 6.1 shows $F_{\text {low }}^{(3)}(\omega, \Omega)$ for a detuning $\delta=\Gamma_{\mathrm{S}} / 2=0.1 U$, when the superconducting correlation on the dot is still strong. In Figure 6.2 the detuning is chosen such that the proximity effect is off-resonance, namely $\delta=2 \Gamma_{\mathrm{S}}=0.4 U$ and the influence of the superconductor is strongly suppressed. Both cases show that the frequencydependent skewness or the Fano factor respectively, fulfills the symmetry $F_{\text {low }}^{(3)}(\omega, \Omega)=F_{\text {low }}^{(3)}(\Omega, \omega)=F_{\text {low }}^{(3)}(\omega, \omega-\Omega)=F_{\text {low }}^{(3)}(\Omega-\omega, \Omega)=F_{\text {low }}^{(3)}(-\omega,-\Omega)$ as a consequence of the time symmetrization of the polyspectrum. In the case when the superconducting correlation on the quantum dot is strong, the maximum in $F_{\text {low }}^{(3)}(\omega, \Omega)$ is not centered at $\omega=\Omega=0$ as it is the case for the finite-frequency noise (see Fig. 4.10). In contrast, when the proximity effect is off-resonance $\Gamma_{S} \ll \delta$, Fig. 6.2, the maximum is centered around zero-frequency. Along the symmetry lines $\Omega=0, \omega=\Omega$ and $\omega=0$ away from the center the skewness gets suppressed in both cases. 


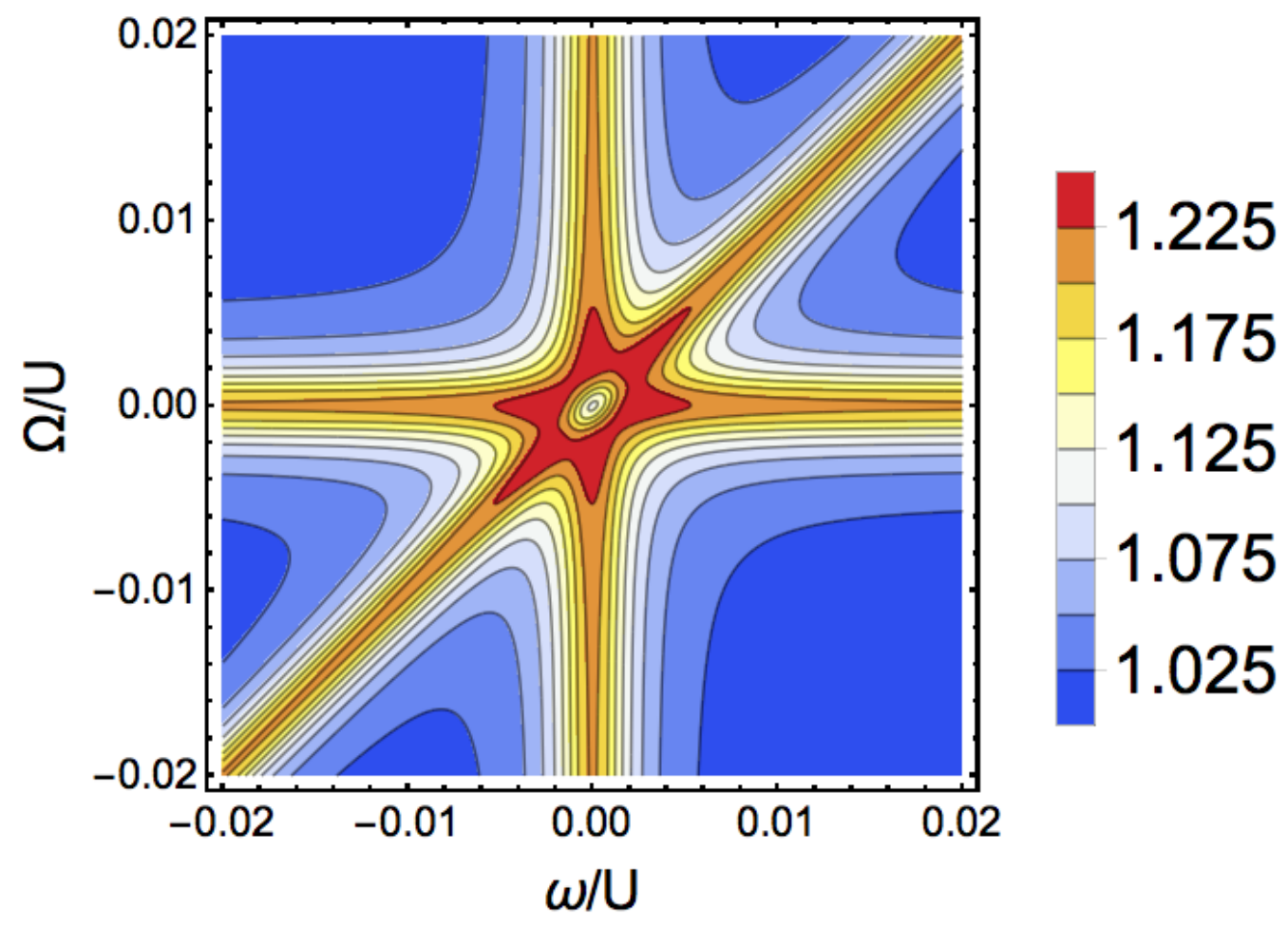

Figure 6.1: Third order Fano factor $F_{\text {low }}^{(3)}(\omega, \Omega)$ in the low-frequency regime for the quantum dot coupled to one normal and one superconducting lead for $\Gamma_{\mathrm{N}}=0.002 U, \Gamma_{\mathrm{S}}=0.2 U$ and $\delta=0.1 U$. 


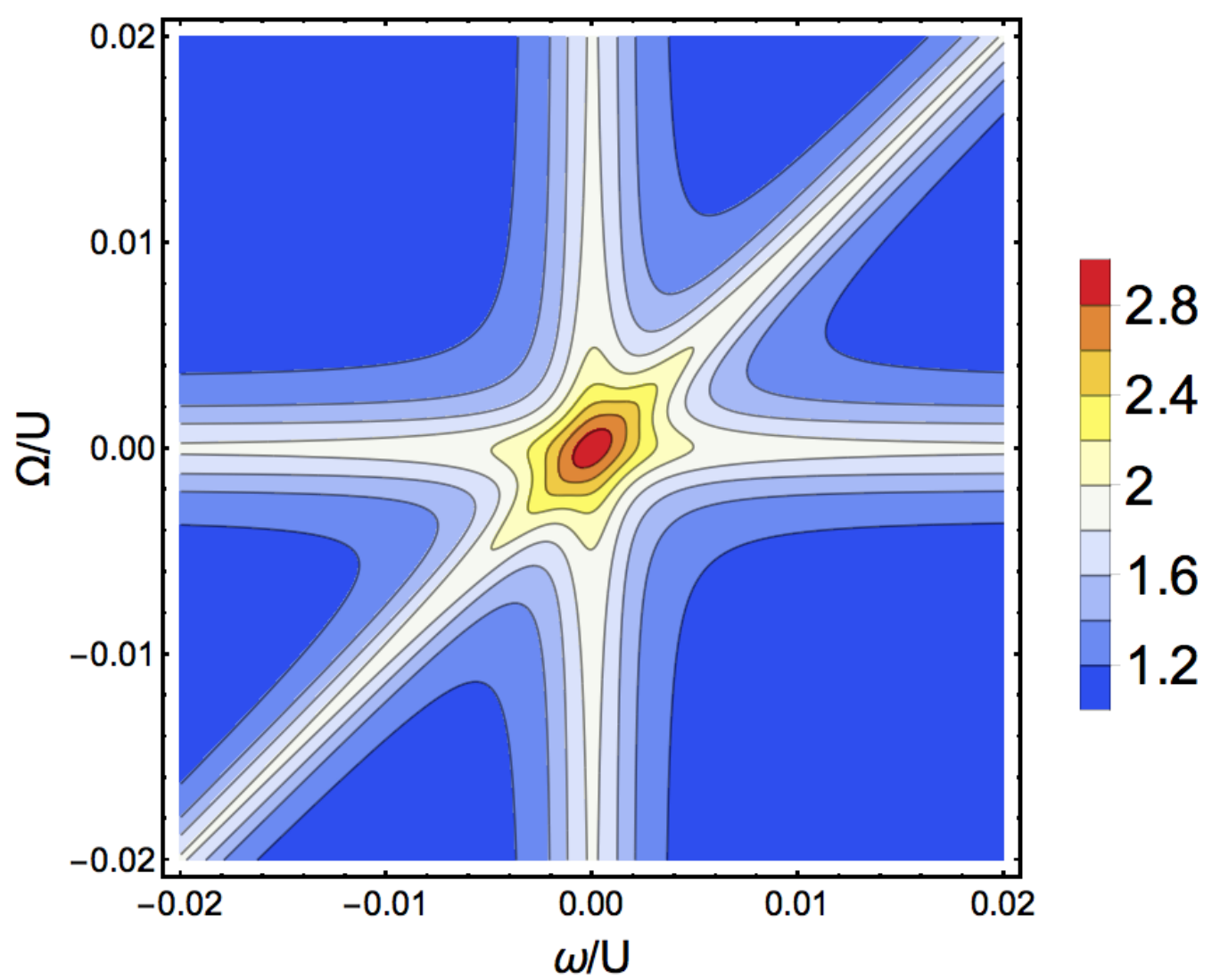

Figure 6.2: Third order Fano factor $F_{\text {low }}^{(3)}(\omega, \Omega)$ in the low-frequency regime for the quantum dot coupled to one normal and one superconducting lead for $\Gamma_{\mathrm{N}}=0.002 U, \Gamma_{\mathrm{S}}=0.2 U$ and $\delta=0.4 U$. 


\subsection{Intermediate-frequency regime}

Now we turn to the intermediate-frequency regime of the skewness, $v_{i} \gg \Gamma_{\mathrm{N}}$, where the noise frequencies become larger than the coupling strength to the normal-conducting lead. As we know from the previous discussion of the finite-frequency noise in Chapter 4 , the spectrum starts to reveal the internal dynamics of the quantum dot in this regime. We find for the frequency dependent skewness in the case of strong coupling to the superconductor $\left(\Gamma_{\mathrm{N}} \ll \Gamma_{\mathrm{S}}\right)$,

$$
\begin{aligned}
\frac{S^{(3)}(\omega, \Omega)}{I} \approx & \frac{S_{\text {low }}^{(3)}(\omega, \Omega)}{I}-\frac{1}{2} \frac{\Gamma_{\mathrm{S}}^{2}}{4 \epsilon_{\mathrm{A}}^{2}} \sum_{i=1}^{3}\left[\frac{\Gamma_{\mathrm{N}}^{2}}{\Gamma_{\mathrm{N}}^{2}+\left(v_{i}-2 \epsilon_{\mathrm{A}}\right)^{2}}\left(1-\frac{v_{i}-2 \epsilon_{\mathrm{A}}}{\epsilon_{\mathrm{A}}}\right)\right. \\
& \left.+\frac{\Gamma_{\mathrm{N}}^{2}}{\Gamma_{\mathrm{N}}^{2}+\left(2 \epsilon_{\mathrm{A}}+v_{i}\right)^{2}}\left(1+\frac{v_{i}+2 \epsilon_{\mathrm{A}}}{\epsilon_{\mathrm{A}}}\right)\right]
\end{aligned}
$$

with $v_{1}=\omega, v_{2}=\Omega$, and $v_{3}=\omega-\Omega$. The first part of Eq. (6.3) is the lowfrequency contribution which has been discussed in the previous section. The remaining part carriers the information of the skewness in the intermediate frequency regime. This result has a similar structure as the finite-frequency noise in the unidirectional transport regime Eq. (4.7). The difference is that in the case of the skewness three noise frequencies are of relevance. The skewness gets suppressed when one of the frequencies is equal to the splitting of the ABSs, $v_{i}=2 \epsilon_{A}$.

For instance, if we set $v_{2}=\Omega=0$, the spectrum $F^{(3)}(\omega, 0)$ exhibits one resonance dip at $\omega=2 \epsilon_{\mathrm{A}}$ as in the finite-frequency noise spectrum. However, the resonance dip scales with a prefactor $\Gamma_{\mathrm{S}}^{2} / 4 \epsilon_{\mathrm{A}}^{2}$, that is double the size of the resonance dip found in the noise spectrum. This is due to the fact that for $v_{2}=\Omega=0$, the remaining frequencies are equal $v_{1}=v_{3}=2 \epsilon_{\mathrm{A}}$. Hence, due to the degeneracy of the frequencies the feature, which indicates the strength of the proximity effect, is enhanced. The same can be found for $v_{1}=\omega=0$.

Now, if we choose the frequencies such that $v_{2}=-v_{1}$, the spectrum exhibits two resonance dips, namely at $\omega=2 \epsilon_{\mathrm{A}}$ and $\omega=\epsilon_{\mathrm{A}}$. Figures 6.3 


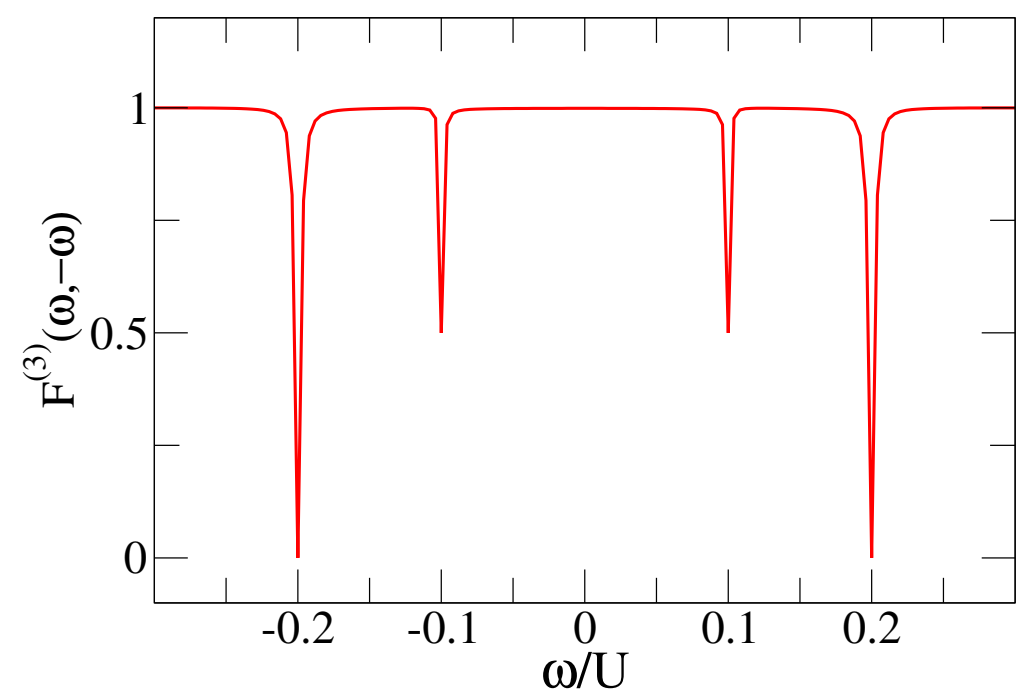

Figure 6.3: Third order Fano factor $F^{(3)}(\omega,-\omega)$ ad a function of $\omega$ with $\Gamma_{\mathrm{N}}=0.002 U, \Gamma_{\mathrm{S}}=0.2 U$ and $\delta=0$.

and 6.4 show results for the third order Fano Factor $F^{(3)}(\omega,-\omega)$ for different values of the detuning $\delta$. Figure 6.3 shows the Fano factor for $\delta=0$, when the proximity effect is on-resonace and the resonance dips have its maximal depth. The resonance dip at $\omega=\epsilon_{\mathrm{A}}$ scales with $\Gamma_{\mathrm{N}}^{2} / 8 \epsilon_{\mathrm{A}}^{2}$ and the second dip at $\omega=2 \epsilon_{\mathrm{A}}$ with $\Gamma_{\mathrm{N}}^{2} / 4 \epsilon_{\mathrm{A}}^{2}$. This can be read off the prefactor in Eq. (6.3). Figure 6.4 shows $F^{(3)}(\omega,-\omega)$ for different values of the detuning $\delta$. The Fano factor behaves as expected from the knowledge of the previous results: the resonance dips become smaller with increasing $\delta$. Hence, they indicate the strength of the induced superconducting correlation on the quantum dot. However, for this choice of the frequencies the feature at $\omega=2 \epsilon_{\mathrm{A}}$ is enhanced in the spectrum of the skewness in contrast to the noise. Consequently, an evidence of the induced superconducting correlation on the quantum dot can be detected even at a rather large detuning $\delta$.

If the frequencies are chosen such that no degeneracy occurs between them, the spectrum has three resonance dip, each with a prefactor $\Gamma_{\mathrm{N}}^{2} / 8 \epsilon_{\mathrm{A}}^{2}$. For example, if we choose $v_{2}=\Omega=-\omega / 2$, the spectrum $F^{(3)}(\omega,-\omega / 2)$ exhibits three resonance dips which appear at $\omega=2 \epsilon_{\mathrm{A}}, \omega=4 \epsilon_{\mathrm{A}}$ and $\omega=\frac{4}{3} \epsilon_{\mathrm{A}}$. 

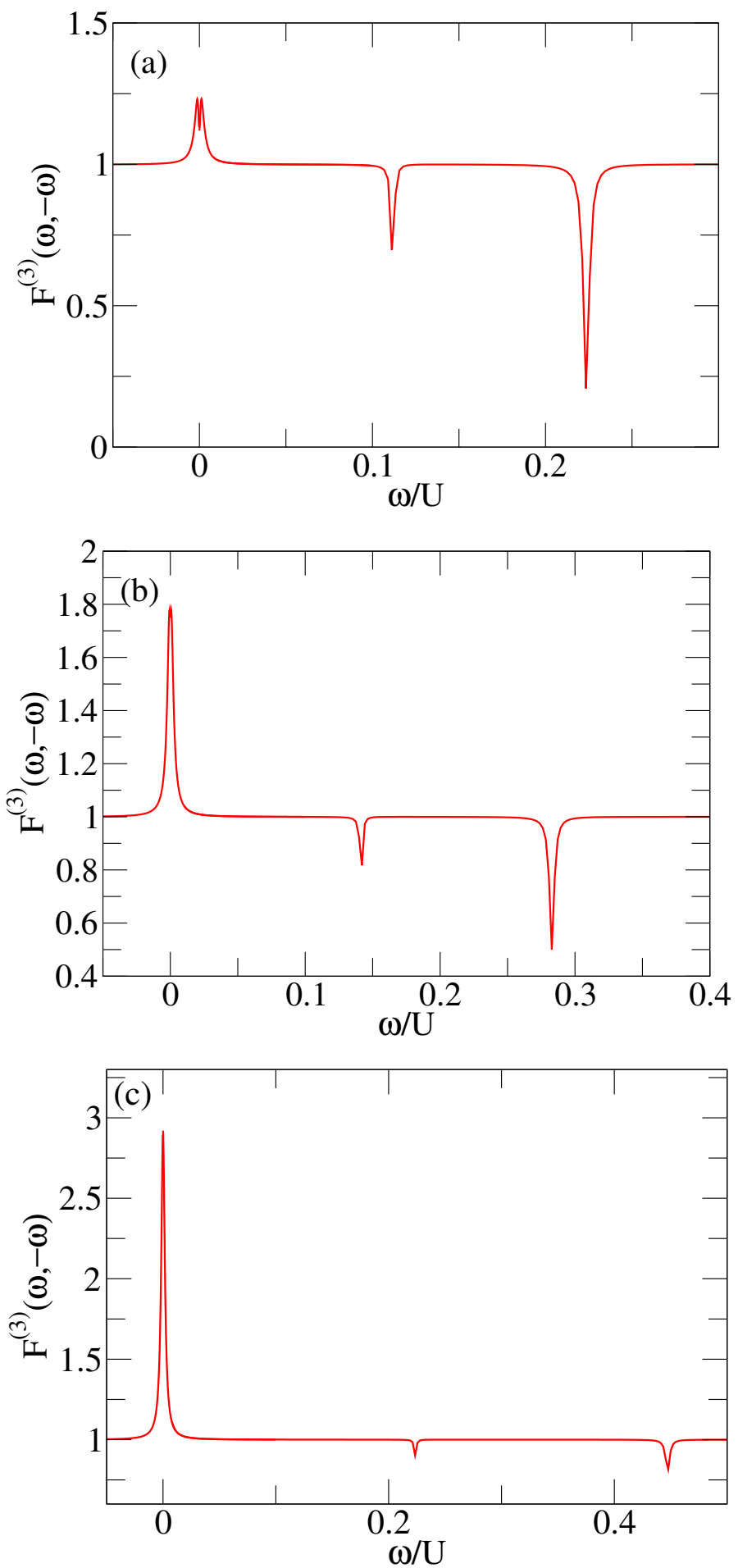

Figure 6.4: Third order Fano factor $F^{(3)}(\omega,-\omega)$ ad a function of $\omega$ with $\Gamma_{\mathrm{N}}=0.002 U, \Gamma_{\mathrm{S}}=0.2 U$ and (a) $\delta=0.1 U$, (b) $\delta=0.2 U$ and (c) $\delta=0.4 U$. 
This case is displayed in Figure 6.5 for $\delta=0$.

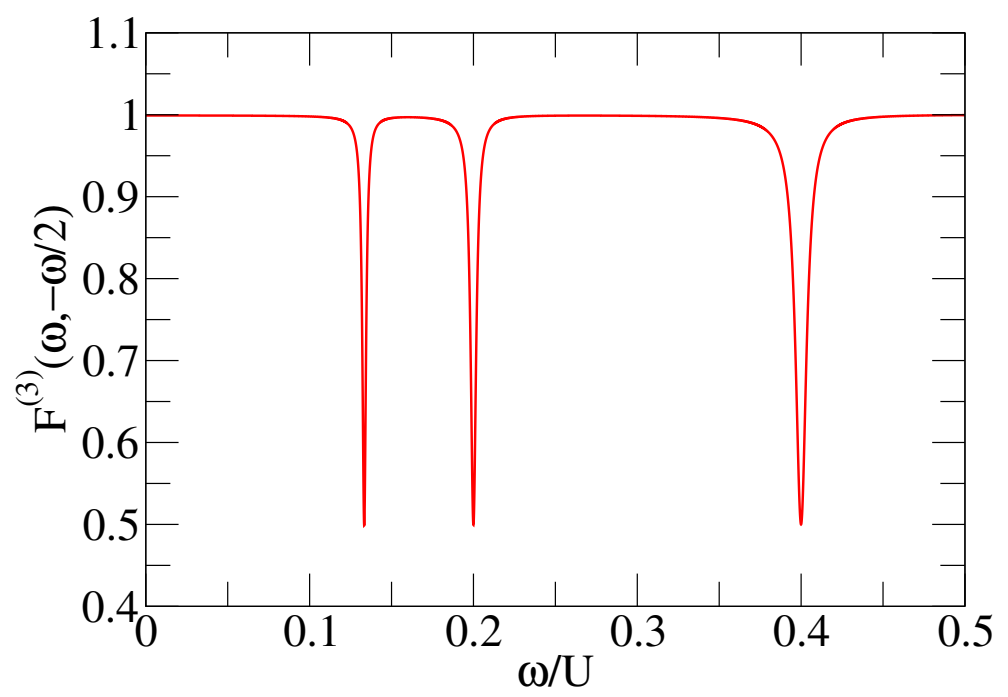

Figure 6.5: Third order Fano factor $F^{(3)}(\omega,-\omega / 2)$ ad a function of $\omega$ with $\Gamma_{\mathrm{N}}=0.002 U, \Gamma_{\mathrm{S}}=0.2 U$ and $\delta=0$.

\subsection{Conclusions}

We have calculated the frequency-dependent skewness of a quantum dot weakly coupled to a normal-conducting lead and strongly to a superconductor lead in the unidirectional transport regime. Whenever one of the frequencies $v_{i}$ is equal the energy which is necessary for a coherent destructive interference of the ABSs, namely $v_{i}=2 \epsilon_{\mathrm{A}}$, a resonance dip occurs in the spectrum of the frequency-dependent skewness. These features are a signature of the coherent exchange of Cooper pairs between quantum dot and superconductor. Position and size of the dips depend on the splitting of the ABSs and hence on the strength of the proximity effect. This is in analogy to the finite-frequency noise, where one dip occurs at $\omega=2 \epsilon_{\mathrm{A}}$. However, the magnitude of the dips can be enhanced if the frequencies are degenerate. This leads to a better visibility of the feature in the spectrum. 


\section{Chapter 7}

\section{Summary and outlook}

In this thesis we have investigated electronic transport out of equilibrium through a nanostructure composed by an interacting single-level quantum dot tunnel-coupled to a superconductor and a normal-conducting lead. In particular, we have studied the current fluctuations, namely the finite-frequency noise and the finite-time FCS in order to reveal the internal dynamics of the system and its characteristic time scales, which are not accessible via Andreev level spectroscopy. We were interested in the subgap-transport regime and considered a strong coupling between the quantum dot and the superconductor, while the dot is only weakly coupled to the normal-conducting lead. For the subgap-transport characteristics of the system, the superconductor can be described by means of an effective Hamiltonian which becomes exact in the limit of an infinite superconducting gap. The effective Hamiltonian still describes well the subgap transport features even for finite values of the gap as long as the temperature is larger than the Kondo temperature related to the Kondo screening by the quasiparticle excitations in the superconductor 94$]$.

In Chapter 3 we have presented a diagrammatic real-time technique which allows us to describe non-equilibrium transport through interacting quantum dots coupled to electronic reservoirs. We have made use of the diagrammaticreal time approach to derive the current and finite-frequency noise through 
the system. In this framework we have set up a theory which allows to obtain the finite-time FCS for non-Markovian processes. This approach enables to calculate current cumulants of arbitrary order beyond the noise. Furthermore, it offers an alternative way to determine the finite-frequency noise, while it also permits to calculate the cross- and autocorrelation functions separately. Lastly, we have introduced multi-time FCS for Markovian systems 62,104 based on the finite-time FCS.

In the main part of Chapter 4 we have presented results for the finitefrequency noise of a quantum dot strongly coupled to a superconductor and weakly coupled to a normal conducting lead. We have studied the finitefrequency noise in different bias and frequency regimes. We found that the finite-frequency noise spectrum reveals the coherent dynamics of the system underlying the proximity effect in the dot, that is the coherent tunneling of Cooper pairs between dot and superconductor. In particular, we identified a resonant feature in the noise spectrum at the frequency of the coherent oscillations of Cooper pairs between dot and superconductor. The magnitude of this feature (a sharp dip in the spectrum) is directly related to the pair amplitude in the dot. Furthermore, in the quantum noise regime the noise spectrum exhibits steps at frequencies equal to the Andreev addition energies, while the height of the steps yields information on the relative coupling of different BCS-like states to the normal lead. The sign and height of these steps can be understood from an analogy to the noise spectrum of a single-level quantum dot coupled to normal leads. The finite-frequency noise spectrum of a single-level quantum dot coupled to normal-conducting leads has also been presented in Chapter 4 . Whether a step in the noise spectrum leads to an increase or a decrease of the total noise depends on the coupling strength of the different excitations: the noise stemming from correlations in the same lead increases at frequencies equal to the excitation energies, while the noise due to correlations between different leads decreases. The analogy is between the correlations between different leads coupled to a single-level quantum dot and correlations between different channels arising 
due to the effectively coupled Andreev levels. Beyond the knowledge of the Andreev addition energies, which can also be obtained from Andreev level spectroscopy by means of a differential conductance measurement 12 22], the finite-frequency noise additionally provides information on the coherent dynamics of the system and its characteristic time scales, and the effective coupling strengths of the different Andreev levels. Hence, the finite-frequency noise spectrum provides a full spectroscopy of the system.

In Chapter 5 we have analyzed the finite-time FCS in terms of a generalized Fano factor for higher order cumulants beyond the noise. We observed that the generalized Fano factor follows a universal behavior independent of the system. The Fano factor $F^{k}(t)$ grows in magnitude and starts to oscillate with increasing cumulant order $k$, before it approaches the long-time limit. However, the magnitude of the Fano factor and the oscillatory characteristics depend on the proximity effect on the dot, namely if the superconducting correlations on the dot are strong or negligible. In the short-time regime, we discovered an oscillatory behavior of the generalized Fano factor with an oscillation frequency equal the splitting of the ABSs. These oscillations become more prominent with increasing cumulant order and depend on the strength of the proximity effect. Hence, also the finite-time FCS elucidate the coherent dynamics underlying the proximity effect, namely the coherent tunneling of Cooper pairs between dot and superconductor.

In Chapter 6 we have investigated the frequency dependent skewness in the high bias regime derived form the theory for multi-time FCS. We found that the bispectrum reveals resonant features whenever one of the frequencies is equal the splitting of the $\mathrm{ABSs}, v_{i}=2 \epsilon_{\mathrm{A}}$ analogously to the finite-frequency noise. We discovered that the magnitude of the resonant feature in the spectrum of the frequency-dependent skewness can be enhanced depending on the choice of the frequencies.

The results obtained in this thesis show the importance of time-dependent current correlations to gain information on the transport processes and elucidate the internal dynamics of a mesoscopic conductor. However, most works 
of noise or FCS, theoretically and experimentally, focus on the long-time limit only. Here we have demonstrated the importance of finite times to reveal the short time dynamics of the system, which carry information on the coherent dynamics.

An experimental realization of a finite-frequency noise measurement as well as the detection of the finite-time FCS is possible with present-day techniques. Current noise spectroscopy in mesoscopic systems has become a standard tool to gain information on the transport processes and internal time scales of mesoscopic conductors [50-59]. In oder to observe the effects in the finite-frequency noise spectrum discussed in this thesis experimentally, the measurement has to be performed in the $\mathrm{GHz}$ frequency range. For example, in recent experiments [14,138 the coupling to the superconductor is $\Gamma_{\mathrm{S}} \approx 50-250 \mathrm{GHz}$. Here, we also derived higher order cumulants in terms of finite-time FCS. Higher order cumulants in counting statistics have been measured experimentally for a quantum dot coupled to normal leads by using a quantum point contact as noninvasive charge detector with fast time response 61, 113, 114. Even the measurement of the frequency-dependent skewness for transport through a quantum dot has been recently realized 58.

Our results can have important ramifications for the study of hybrid superconducting-normal structure with quantum dots in the future and might motivate the measurement of finite-frequency noise and the higher order cumulants in hybrid systems.

The logical continuation would be to study the current fluctuations in more complex hybrid-superconductor normal structures as for example a double quantum dot. Here, it might be interesting to see how parameters like the intra-dot interaction or coupling influence the internal dynamics of the system. Also an inclusion of spin-orbit coupling and a magnetic field is possible, since we consider two orbital levels.

Beside this, a double quantum dot is an ideal tool to generate all types of superconducting correlations in a single device via bias, gate voltage and inhomogeneous magnetic fields [8]. Different types of superconducting cor- 
relations can be defined in terms of order parameters, namely for even-/odd frequency singlet/triplet correlations. Due to the small number of degrees of freedom this system is an ideal tool to exhibit unconventional pairing and hence gain fundamental insight in unconventional pairing via Josephson or Andreev spectroscopy. However, it might be of importance to study the finite-frequency noise to reveal the internal dynamics of the system and to gain further information on the unconventional superconducting correlations. 
Appendices 



\section{Appendix A}

\section{Scattering approach for finite-frequency noise}

\section{A.1 Scattering formalism}

The scattering approach is a very powerful tool to address the transport properties of a mesoscopic system knowing its scattering properties. The system can be either at equilibrium or out-of-equilibrium. However, the scattering formalism allows only to obtain the transport properties of noninteracting systems. Nevertheless, its an established method to extract the transport properties of a mesoscopic conductor like a quantum dot 32. Here, we like to present the scattering formalism for a non-interacting single level quantum dot coupled to two normal conducting leads and how to determine the finite-frequency noise by using the scattering formalism. This has been accomplished in Ref. 36, 37.

Results for the symmetrized finite-frequency noise for a single-level quantum dot coupled to normal-conducting leads are discussed in Chapter 4. The basic idea of the scattering approach is to examine a small region contacted to some reservoirs. The transport properties of such a system are then defined by a scattering matrix $\mathrm{S}$ which gives the amplitudes of electron scattering between different channels in different reservoirs. 
In the simple model of a dot coupled to normal leads the scattering matrix takes the form

$$
S(E)=-1+i g(E)\left(\begin{array}{cc}
\Gamma_{L} & \sqrt{\Gamma_{\mathrm{L}} \Gamma_{\mathrm{R}}} \\
\sqrt{\Gamma_{\mathrm{L}} \Gamma_{\mathrm{R}}} & \Gamma_{\mathrm{R}}
\end{array}\right)
$$

where $g(E)$ is the Breit-Wigner resonance formed by the dot,

$$
g(E)=\frac{1}{E-\epsilon+i \Gamma / 2} .
$$

The scattering matrix $S(E)$ is a function of the energy of the scattered electron. In order to find the expression for the symmetrized finite-frequency noise spectrum we define first the non-symmetrized noise in the single reservoir as

$$
C_{\alpha \alpha^{\prime}}(\omega)=\int_{-\infty}^{\infty} d t e^{i \omega t}\left\langle\delta \hat{I}_{\alpha}(t) \delta \hat{I}_{\alpha^{\prime}}(0)\right\rangle
$$

where $\alpha$ and $\alpha^{\prime}$ denote the leads which transport the current between the electronic reservoirs and the system and $\delta \hat{I}_{\alpha}=\hat{I}_{\alpha}-\left\langle\hat{I}_{\alpha}\right\rangle$ with the current operator $\hat{I}_{\alpha}$. The time-dependent current operator is a function of creation and annihilation operators of the electrons in reservoir $\alpha$, namely $\hat{a}_{\alpha}^{(\dagger)}$. The general expression of the current operator is given by

$$
\hat{I}_{\alpha}(t)=\frac{e}{h} \sum_{\gamma, \gamma^{\prime}} \int d E d E^{\prime} e^{i\left(E-E^{\prime}\right) t / \hbar} A_{\gamma \gamma^{\prime}}\left(\alpha ; E, E^{\prime}\right) \hat{a}_{\gamma}^{\dagger}(E) \hat{a}_{\gamma^{\prime}}\left(E^{\prime}\right)
$$

with

$$
A_{\gamma \gamma^{\prime}}\left(\alpha ; E, E^{\prime}\right)=\delta_{\gamma \gamma^{\prime}} \delta_{\alpha \gamma}-S_{\alpha \gamma}^{*}(E) S_{\alpha \gamma^{\prime}}\left(E^{\prime}\right)
$$

the elements of the scattering matrix of the system. In the case of a quantum dot coupled to two leads, $\alpha=\mathrm{L}, \mathrm{R}$, we can distinguish between two types of correlation functions Eq. A.3. For $\alpha=\alpha^{\prime}$ we denote $C_{\mathrm{LL}}$ and $C_{\mathrm{RR}}$ as auto-correlation functions. If $\alpha \neq \alpha^{\prime}$ we are left with the so-called crosscorrelation functions $C_{\mathrm{LR}}$ and $C_{\mathrm{RL}}$. Considering the net current through the device, $\hat{I}=\left(\hat{I}_{\mathrm{L}}-\hat{I}_{\mathrm{R}}\right) / 2$, one can write the finite-frequency noise as

$$
C(\omega)=\frac{1}{4}\left(C_{\mathrm{LL}}(\omega)+C_{\mathrm{RR}}(\omega)-C_{\mathrm{LR}}(\omega)-C_{\mathrm{RL}}(\omega)\right) .
$$


The symmetrized version of the noise spectrum is determined by, $C_{\alpha \alpha^{\prime}}^{\mathrm{sym}}(\omega)=$ $\left(C_{\alpha \alpha^{\prime}}(\omega)+C_{\alpha \alpha^{\prime}}(-\omega)\right) / 2$. The non-symmetrized noise $\left.\mathrm{A} .3\right)$ can be written in terms of the scattering matrix elements and yields

$$
C_{\alpha \alpha^{\prime}}(\omega)=\frac{e^{2}}{2 \pi} \int d E \sum_{\gamma \gamma^{\prime}} F_{\gamma \gamma^{\prime}}^{\alpha \alpha^{\prime}}(E, \omega) f_{\gamma}(E+\omega)\left(1-f_{\gamma^{\prime}}(E)\right)
$$

where $f_{\gamma^{\prime}}(E)=1 / 1+e^{\left(E-\mu_{\gamma}\right) / k_{B} T}$ is the Fermi distribution in reservoir $\gamma$ held at the chemical potential $\mu_{\gamma}$ and $F_{\gamma \gamma^{\prime}}^{\alpha \alpha^{\prime}}=A_{\gamma \gamma^{\prime}}(\alpha ; E+\omega, E) A_{\gamma^{\prime} \gamma}\left(\alpha^{\prime} ; E, E+\omega\right)$.

The correlation functions contributing to the current noise Eq. A.7) are each given as a sum of four processes, namely the inter-lead contributions and the intra-lead ones. Taking this into consideration the symmetrized current noise spectrum can be determined by

$$
C(\omega)=\int_{-\infty}^{\infty} \frac{d E}{4 \pi} \sum_{\gamma \gamma^{\prime}} F_{\gamma \gamma^{\prime}}(E, \omega) f_{\gamma}(E+\omega)\left[1-f_{\gamma^{\prime}}(E)\right]+(\omega \rightarrow-\omega)
$$

where

$$
\begin{gathered}
F_{\mathrm{LL}}(E, \omega)=\left|1-S_{\mathrm{LL}}^{*}(E+\omega) S_{\mathrm{LL}}(E)+S_{\mathrm{RL}}^{*}(E+\omega) S_{\mathrm{RL}}(E)\right|^{2} \\
F_{\mathrm{LR}}(E, \omega)=\left|S_{\mathrm{LL}}^{*}(E+\omega) S_{\mathrm{LR}}(E)+S_{\mathrm{RL}}^{*}(E+\omega) S_{\mathrm{RR}}(E)\right|^{2}
\end{gathered}
$$

The other correlations $F_{\mathrm{RR}}(E, \omega)$ and $F_{\mathrm{RL}}(E, \omega)$ are obtained by interchanging $\mathrm{L} \leftrightarrow \mathrm{R}$ in the expressions above.

\section{A.2 Results}

In this section we present analytic results for the current and finite-frequency noise through a non-interacting single-level quantum dot coupled to normalconducting leads obtained by the scattering formalism introduced above.

The current through a non-interacting single level quantum dot with normal-conducting leads leads yields

$$
I=\frac{2 \Gamma_{\mathrm{L}} \Gamma_{\mathrm{R}}\left(f_{\mathrm{L}}^{+}(\epsilon) f_{\mathrm{R}}^{-}(\epsilon)-f_{\mathrm{R}}^{+}(\epsilon) f_{\mathrm{L}}^{-}(\epsilon)\right)}{\Gamma_{\mathrm{L}}+\Gamma_{\mathrm{R}}}
$$


with the total tunnel coupling strength $\Gamma=\Gamma_{\mathrm{L}}+\Gamma_{\mathrm{R}}$ and $f^{-}(\epsilon)=\left(1-f^{+}(\epsilon)\right)$. In the unidirectional transport regime, namely when the applied bias voltage $V\left(\mu_{\mathrm{L}}=V / 2=-\mu_{\mathrm{R}}\right)$ is the largest energy scale in the system, the Fermi functions can be approximated by $f_{\mathrm{L}}^{+}=1$ and $f_{\mathrm{R}}^{+}=0$. The current in the unidirectional transport regime is given by

$$
I=\frac{2 \Gamma_{\mathrm{L}} \Gamma_{\mathrm{R}}}{\Gamma_{\mathrm{L}}+\Gamma_{\mathrm{R}}} .
$$

The finite-frequency noise can be obtained by Eq. (A.8). In particular, one has to find first solutions for the correlations $F_{\gamma \gamma^{\prime}}$. In the zero-frequency limit $\omega=0$ the symmetrized current noise is given by

$$
\begin{aligned}
C(\omega=0)= & \frac{8 \Gamma_{\mathrm{L}}^{2} \Gamma_{\mathrm{R}}^{2}}{\Gamma^{3}}\left(f_{\mathrm{L}}^{+}(\epsilon) f_{\mathrm{L}}^{-}(\epsilon)+f_{\mathrm{R}}^{+}(\epsilon) f_{\mathrm{R}}^{-}(\epsilon)\right)+ \\
& \frac{4 \Gamma_{\mathrm{L}}^{3} \Gamma_{\mathrm{R}}+4 \Gamma_{\mathrm{R}}^{3} \Gamma_{\mathrm{L}}}{\Gamma^{3}}\left(f_{\mathrm{L}}^{+}(\epsilon) f_{\mathrm{R}}^{-}(\epsilon)+f_{\mathrm{R}}^{+}(\epsilon) f_{\mathrm{L}}^{-}(\epsilon)\right) .
\end{aligned}
$$

The noise in the low-frequency regime results in

$$
\begin{aligned}
C_{\text {low }}(\omega)= & {\left[\left(8 \Gamma_{\mathrm{L}}^{2} \Gamma_{\mathrm{R}}^{2}+2 \omega^{2} \Gamma_{\mathrm{L}}^{2}\right) f_{\mathrm{L}}^{+}(\epsilon) f_{\mathrm{L}}^{-}(\epsilon)+\left(8 \Gamma_{\mathrm{L}}^{2} \Gamma_{\mathrm{R}}^{2}+2 \omega^{2} \Gamma_{\mathrm{R}}^{2}\right) f_{\mathrm{R}}^{+}(\epsilon) f_{\mathrm{R}}^{-}(\epsilon)+\right.} \\
& \left(4 \Gamma_{\mathrm{L}}^{3} \Gamma_{\mathrm{R}}+4 \Gamma_{\mathrm{L}} \Gamma_{\mathrm{R}}^{3}+2 \Gamma_{\mathrm{L}} \Gamma_{\mathrm{R}} \omega^{2}\right) f_{\mathrm{L}}^{+}(\epsilon) f_{\mathrm{R}}^{-}(\epsilon)+ \\
& \left.\left(4 \Gamma_{\mathrm{L}}^{3} \Gamma_{\mathrm{R}}+4 \Gamma_{\mathrm{L}} \Gamma_{\mathrm{R}}^{3}+2 \Gamma_{\mathrm{R}} \Gamma_{\mathrm{L}} \omega^{2}\right) f_{\mathrm{R}}^{+}(\epsilon) f_{\mathrm{L}}^{-}(\epsilon)\right] /\left(\Gamma^{3}+\omega^{2} \Gamma\right) .
\end{aligned}
$$

In the high-frequency regime we find

$$
\begin{aligned}
C_{\text {high }}(\omega)= & \frac{1}{2} \frac{\Gamma_{\mathrm{L}}^{2}}{\Gamma}\left[f_{\mathrm{L}}^{+}(\epsilon) f_{\mathrm{L}}^{-}(\epsilon-\omega)+f_{\mathrm{L}}^{+}(\epsilon+\omega) f_{\mathrm{L}}^{-}(\epsilon)\right]+ \\
& \frac{1}{2} \frac{\Gamma_{\mathrm{R}}^{2}}{\Gamma}\left[f_{\mathrm{R}}^{+}(\epsilon) f_{\mathrm{R}}^{-}(\epsilon-\omega)+f_{\mathrm{R}}^{+}(\epsilon+\omega) f_{\mathrm{R}}^{-}(\epsilon)\right]+ \\
& \frac{1}{2} \frac{\Gamma_{\mathrm{L}} \Gamma_{\mathrm{R}}}{\Gamma}\left[f_{\mathrm{L}}^{+}(\epsilon) f_{\mathrm{R}}^{-}(\epsilon-\omega)+f_{\mathrm{L}}^{+}(\epsilon+\omega) f_{\mathrm{R}}^{-}(\epsilon)\right]+ \\
& \frac{1}{2} \frac{\Gamma_{\mathrm{L}} \Gamma_{\mathrm{R}}}{\Gamma}\left[f_{\mathrm{R}}^{+}(\epsilon) f_{\mathrm{L}}^{-}(\epsilon-\omega)+f_{\mathrm{R}}^{+}(\epsilon+\omega) f_{\mathrm{L}}^{-}(\epsilon)\right]+(\omega \rightarrow-\omega) .
\end{aligned}
$$




\section{Appendix B}

\section{Examples for diagrams}

In this part of the Appendix we present practical examples on how to obtain diagrams contributing to the different types of self-energy kernels using diagrammatic rules introduced in Chapter 3 for the finite-frequency noise, Sec. 3.4 and the finite-time FCS, section 3.5 .

\section{B.1 Diagrams contributing to the finite-frequency noise}

In this section we show how to obtain the elements $W_{\chi_{2} \chi_{2}^{\prime}}^{\chi_{1} \chi_{1}^{\prime}}(\omega)$ of the frequencydependent kernels contributing to the finite-frequency noise on the example of the transition $|\sigma\rangle \rightarrow|+\rangle$. The contributing diagrams to the element $W_{+\sigma}^{+\sigma}(\omega)$ are displayed in Fig. B.1. Note, that we have to take into account that the states $| \pm\rangle$ are a superposition of the empty $|0\rangle$ and doubly $|d\rangle$ occupied state and hence, 4 diagrams contribute to the kernel element $W_{+\sigma}^{+\sigma}(\omega)$. Applying the diagrammatic rules introduced in Sec. 3.4, we obtain the following 
integral expression for the kernel element $W_{+, \sigma}^{+, \sigma}(\omega)$,

$$
\begin{aligned}
& W_{+\sigma}^{+\sigma}(\omega)=(-i)(-1) \int \frac{d \varpi}{2 \pi} \frac{1}{2}\left(1-\frac{\delta}{2 \epsilon_{\mathrm{A}}}\right) \frac{\Gamma_{\mathrm{N}}}{2} f^{-}(\varpi) \times \\
& {\left[\frac{1}{\varpi-\left(E_{\sigma}-E_{+}\right)-\omega+i 0^{+}}+\frac{1}{-\varpi+\left(E_{\sigma}-E_{+}\right)-\omega+i 0^{+}}\right] } \\
&+(-i)(-1) \int \frac{d \varpi}{2 \pi} \frac{1}{2}\left(1+\frac{\delta}{2 \epsilon_{\mathrm{A}}}\right) \frac{\Gamma_{\mathrm{N}}}{2} f^{+}(\varpi) \times \\
& {\left[\frac{1}{\varpi-\left(E_{+}-E_{\sigma}\right)-\omega+i 0^{+}}+\frac{1}{-\varpi+\left(E_{+}-E_{\sigma}\right)-\omega+i 0^{+}}\right] }
\end{aligned}
$$

with the Fermi function $f^{+}(\varpi)=\left[1+e^{\beta(\varpi-\mu)}\right]^{-1}$ and $f^{-}(\varpi)=\left(1-f^{+}(\varpi)\right)$. At this stage it is useful to use the relation,

$$
\frac{1}{x+i 0^{+}}=\frac{P}{x}-i \pi \delta(x)
$$

which decomposes the fraction in terms of the Cauchy principle value $P$ and a delta function. Introducing this relation, the integral yields

$$
\begin{aligned}
\Rightarrow W_{+\sigma}^{+\sigma}(\omega)= & i \int \frac{d \varpi}{2 \pi} \frac{1}{2}\left(1-\frac{\delta}{2 \epsilon_{\mathrm{A}}}\right) \frac{\Gamma_{\mathrm{N}}}{2} f^{-}(\varpi) \times \\
& {\left[\frac{P}{\varpi-\left(E_{-,-}+\omega\right)}-\frac{P}{\varpi-\left(E_{-,-}-\omega\right)}-\right.} \\
& \left.i \pi\left(\delta\left(\varpi-\left(E_{-,-}+\omega\right)\right)+i \delta\left(\varpi-\left(E_{+,+}-\omega\right)\right)\right)\right] \\
& +i \int \frac{d \varpi}{2 \pi} \frac{1}{2}\left(1+\frac{\delta}{2 \epsilon_{\mathrm{A}}}\right) \frac{\Gamma_{\mathrm{N}}}{2} f^{+}(\varpi) \times \\
& {\left[\frac{P}{\varpi-\left(E_{+,+}+\omega\right)}-\frac{P}{\varpi-\left(E_{+,+}-\omega\right)}-\right.} \\
& \left.i \pi\left(\delta\left(\varpi-\left(E_{+,+}+\omega\right)\right)+\delta\left(\varpi-\left(E_{+,+}-\omega\right)\right)\right)\right] .
\end{aligned}
$$

While the terms with the delta function can be solved immediately, we have to calculate the principle value separately. In particular, we have to solve an integral of type, $\int \frac{f(\varpi-\mu)}{\varpi-\epsilon} d \varpi$. This integral can be evaluated in terms of a contour integral under consideration of the residue theorem. The final result yields,

$$
\int \frac{f(\varpi-\mu)}{\varpi-\epsilon} d \varpi=\Re\left(\Psi\left(\frac{1}{2}+\frac{i \beta}{2 \pi}(\epsilon-\mu)\right)\right)-\Psi\left(\frac{1}{2}+\frac{\beta E_{\mathrm{C}}}{2 \pi}\right)
$$


Diagrams contributing to the

with the digamma function $\Psi$ and $E_{\mathrm{C}}$ is a cutoff energy introduced to secure that the integral converges. The digamma function $\Psi(z)$ can be computed in the complex plane,

$$
\Psi(z)=-\gamma+\sum_{n=0}^{\infty}\left(\frac{1}{n+1}-\frac{1}{n+z}\right)
$$

and hold the relations

$$
\Psi(x)-\Psi(y)=\sum_{n=0}^{\infty}\left(\frac{-1}{n+x}+\frac{1}{n+y}\right)
$$

and

$$
\Psi(\bar{z})=\overline{\Psi(z)}
$$

Further details about the digamma function and its properties can be found in Ref. [162]. Taking into account Eq. (B.1), we get,

$$
\begin{aligned}
\Rightarrow W_{+\sigma}^{+\sigma}(\omega)= & \frac{\Gamma_{\mathrm{N}}}{4}\left(1-\frac{\delta}{2 \epsilon_{\mathrm{A}}}\right)\left[f^{-}\left(E_{-,-}+\omega\right)+f^{-}\left(E_{-,-}-\omega\right)\right. \\
& -\frac{i}{\pi}\left(\Re\left(\Psi\left(\frac{1}{2}+\frac{i \beta}{2 \pi}\left(E_{-,-}+\omega\right)\right)\right)-\right. \\
& \left.\left.\Re\left(\Psi\left(\frac{1}{2}+\frac{i \beta}{2 \pi}\left(E_{-,-}-\omega\right)\right)\right)\right)\right]+ \\
& \frac{\Gamma_{\mathrm{N}}}{4}\left(1+\frac{\delta}{2 \epsilon_{\mathrm{A}}}\right)\left[f^{+}\left(E_{+,+}+\omega\right)+f^{+}\left(E_{+,+}-\omega\right)+\right. \\
& \frac{i}{\pi}\left(\Re\left(\Psi\left(\frac{1}{2}+\frac{i \beta}{2 \pi}\left(E_{+,+}+\omega\right)\right)\right)-\right. \\
& \left.\left.\Re\left(\Psi\left(\frac{1}{2}+\frac{i \beta}{2 \pi}\left(E_{+,+}-\omega\right)\right)\right)\right)\right] .
\end{aligned}
$$

Including also the bias by shifting the energies, the final expression for the self-energy kernel element $W_{+\sigma}^{+\sigma}(\omega)$ results in 


$$
\begin{aligned}
W_{+\sigma}^{+\sigma}(\omega)= & \frac{\Gamma_{\mathrm{N}}}{4}\left(1-\frac{\delta}{2 \epsilon_{\mathrm{A}}}\right)\left[f^{-}\left(E_{-,-}+\omega-\mu\right)+f^{-}\left(E_{-,-}-\omega-\mu\right)\right. \\
& -\frac{i}{\pi}\left(\Re\left(\Psi\left(\frac{1}{2}+\frac{i \beta}{2 \pi}\left(E_{-,-}+\omega-\mu\right)\right)\right)\right. \\
& \left.\left.-\Re\left(\Psi\left(\frac{1}{2}+\frac{i \beta}{2 \pi}\left(E_{-,-}-\omega-\mu\right)\right)\right)\right)\right]+ \\
& \frac{\Gamma_{\mathrm{N}}}{4}\left(1+\frac{\delta}{2 \epsilon_{\mathrm{A}}}\right)\left[f^{+}\left(E_{+,+}+\omega-\mu\right)+f^{+}\left(E_{+,+}-\omega-\mu\right)\right. \\
& +\frac{i}{\pi}\left(\Re\left(\Psi\left(\frac{1}{2}+\frac{i \beta}{2 \pi}\left(E_{+,+}+\omega-\mu\right)\right)\right)-\right. \\
& \left.\Re\left(\Psi\left(\left(\frac{1}{2}+\frac{i \beta}{2 \pi}\left(E_{+,+}-\omega-\mu\right)\right)\right)\right)\right] .
\end{aligned}
$$

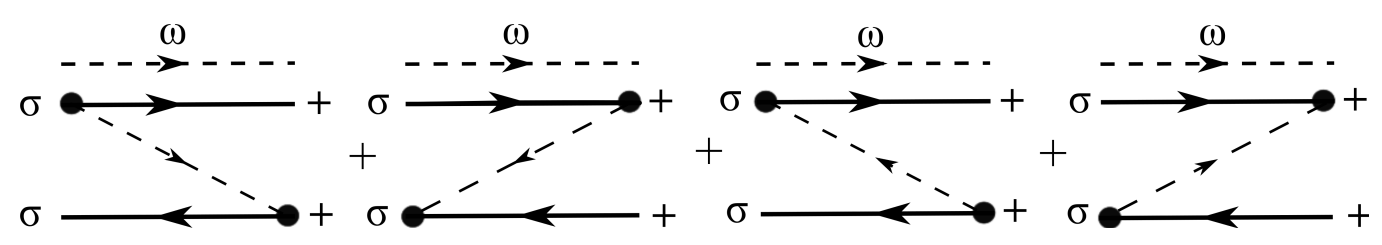

Figure B.1: Diagrams contributing to the kernel element $W_{+\sigma}^{+\sigma}(\omega)$.

The next type of diagrams are these contributing to the current kernels. To calculate the current kernels in first order we have to replace one tunnel vertex by a current vertex, sum over all possible realizations of replacement and use the rules introduced in Sec. 3.4. As introduced in Sec. 3.4 there are two different types of current vertices at finite-frequencies, namely $W_{I>\chi_{2} \chi_{2}^{\prime}}^{\chi_{1} \chi_{1}^{\prime}}(\omega)$ and $W_{I<\chi_{2} \chi_{2}^{\prime}}^{\chi_{1} \chi_{1}^{\prime}}(\omega)$. In the limit of zero-frequencies, the relation $\boldsymbol{W}_{I>}(\omega=0)=\boldsymbol{W}_{I<}(\omega=0)=\boldsymbol{W}^{I}(\omega=0)$ holds. Figure B.2 (a) shows the diagrams contributing to the kernel element $W_{I>\chi_{2} \chi_{2}^{\prime}}^{\chi_{1} \chi_{1}^{\prime}}(\omega)$ and (b) to $W_{I<\chi_{2} \chi_{2}^{\prime}}^{\chi_{1} \chi_{1}^{\prime}}(\omega)$. Finally, using the diagrammatic rules and the result of the 
(a)

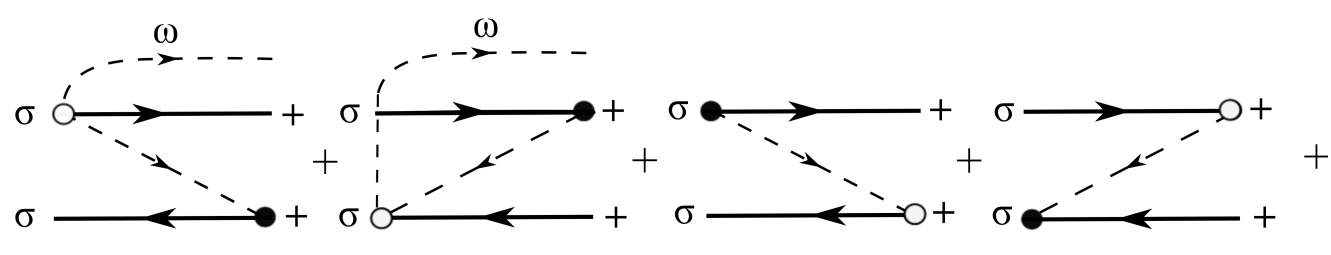

$\omega$

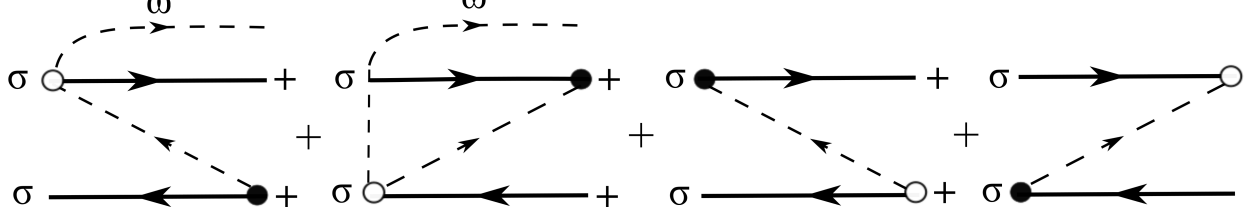

(b)

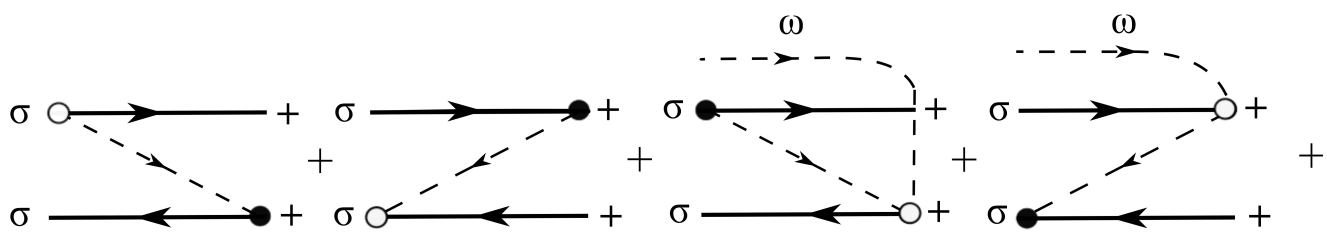

$\omega$

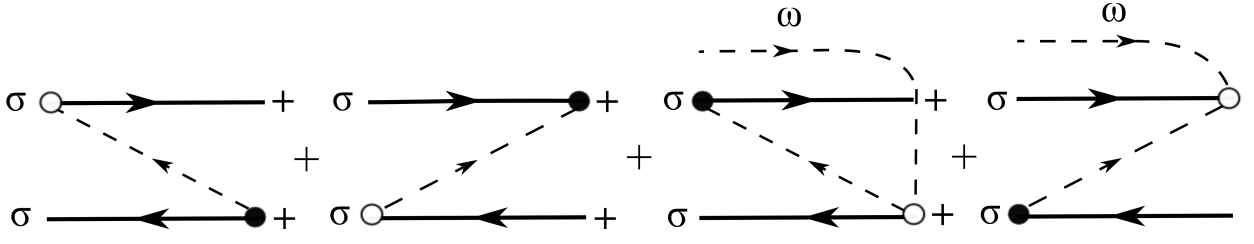

Figure B.2: (a) Diagrams contributing to the kernel element $W_{I>+\sigma}^{+\sigma}(\omega)$ and (b) all diagrams contributing to the kernel element $W_{I<+\sigma}^{+\sigma}(\omega)$. 
integral expression Eq. (B.1) we obtain for the kernel element $W_{I>\chi_{2} \chi_{2}^{\prime}}^{\chi_{1} \chi_{1}^{\prime}}(\omega)$, .

$$
\begin{aligned}
W_{I>+\sigma}^{+\sigma}(\omega) & =-\frac{\Gamma_{\mathrm{N}}}{4}\left(1-\frac{\delta}{2 \epsilon_{\mathrm{A}}}\right)\left[f^{-}\left(E_{-,-}+\omega-\mu\right)+f^{-}\left(E_{-,-}-\omega-\mu\right)\right. \\
& +2 f^{-}\left(E_{-,-}-\mu\right)-\frac{i}{\pi}\left(\Re\left(\frac{1}{2}+\frac{i \beta}{2 \pi}\left(E_{-,-}+\omega-\mu\right)\right)\right. \\
& \left.\left.-\Re\left(\frac{1}{2}+\frac{i \beta}{2 \pi}\left(E_{-,-}-\omega-\mu\right)\right)\right)\right] \\
& +\frac{\Gamma_{\mathrm{N}}}{4}\left(1+\frac{\delta}{2 \epsilon_{\mathrm{A}}}\right)\left[f^{+}\left(E_{+,+}+\omega-\mu\right)+f^{+}\left(E_{+,+}-\omega-\mu\right)\right. \\
& \left.+\frac{i}{\pi}\left(\Re\left(\frac{1}{2}+\frac{i \beta}{2 \pi}\left(E_{+,+}+\omega-\mu\right)\right)-\Re\left(\frac{1}{2}+\frac{i \beta}{2 \pi}\left(E_{+,+}-\omega-\mu\right)\right)\right)\right] .
\end{aligned}
$$

Analogously, we can calculate the element $W_{I<+\sigma}^{+\sigma}(\omega)$ and find that they are equal

$$
W_{I<+\sigma}^{+\sigma}(\omega)=W_{I>+\sigma}^{+\sigma}(\omega)
$$

Note, that this result is not universal and does not hold for all kernel elements.

Finally, we also need to calculate the contribution, where both current operators are in the same block. Therefore we need to replace two tunnel vertices by two current vertices and connect them with an external line transporting the energy $\omega$. For the particular system we find for the example

$$
W_{I I+\sigma}^{+\sigma}(\omega)=W_{+\sigma}^{+\sigma}(\omega)
$$

In the same way as shown for this example all contributing kernel elements can be evaluated, including the transition rates that involve off-diagonal reduced density matrix elements. 


\section{B.2 Diagrams contributing to the finite-time FCS}

Here, we want to provide one example on how to obtain the kernel elements for the kernel $\Gamma(\chi, z)$ introduced in Sec. 3.5. We choose as in the previous examples the transition $|\sigma\rangle \rightarrow|+\rangle$. In first order in the tunnel coupling strength the kernel element is given by

$$
z \Gamma_{+\sigma}^{+\sigma}\left(\chi_{\mathrm{N}}, z\right)=\tilde{W}_{+\sigma}^{+\sigma}\left(\chi_{\mathrm{N}}, z=0\right)-\tilde{W}_{+\sigma}^{+\sigma}\left(\chi_{\mathrm{N}}, z\right)
$$

with the kernel $\tilde{W}$ representing these diagrams where one tunnel vertex in the counting interval $(\chi \neq 0)$ gets contracted with a vertex in the noncounting interval $(\chi=0)$. The diagrams contributing to the kernel element $\tilde{W}_{+\sigma}^{+\sigma}\left(\chi_{\mathrm{N}}, z\right)$ are shown in Fig. B.3. After applying diagrammatic rules the kernel element $\tilde{W}_{+\sigma}^{+\sigma}\left(\chi_{\mathrm{N}}, z\right)$ yields

$$
\begin{aligned}
\tilde{W}_{+\sigma}^{+\sigma}\left(\chi_{\mathrm{N}}, z\right) & =\frac{\Gamma_{\mathrm{N}} e^{i \chi_{\mathrm{N}} / 2}}{4}\left(1+\frac{\delta}{2 \epsilon_{\mathrm{A}}}\right)\left[f^{+}\left(E_{+,+}+i z-\mu\right)+f^{+}\left(E_{+,+}-i z-\mu\right)\right. \\
& \left.+\frac{i}{\pi}\left(\Re\left(\frac{1}{2}+\frac{i \beta}{2 \pi}\left(E_{+,+}-i z-\mu\right)\right)-\Re\left(\frac{1}{2}+\frac{i \beta}{2 \pi}\left(E_{+,+}+i z-\mu\right)\right)\right)\right] \\
& +\frac{\Gamma_{\mathrm{N}} e^{-i \chi_{\mathrm{N}} / 2}}{4}\left(1-\frac{\delta}{2 \epsilon_{\mathrm{A}}}\right)\left[f^{-}\left(E_{-,-}+i z-\mu\right)+f^{-}\left(E_{-,-}-i z-\mu\right)\right. \\
& \left.-\frac{i}{\pi}\left(\Re\left(\frac{1}{2}+\frac{i \beta}{2 \pi}\left(E_{-,-}-i z-\mu\right)\right)-\Re\left(\frac{1}{2}+\frac{i \beta}{2 \pi}\left(E_{-,-}+i z-\mu\right)\right)\right)\right]
\end{aligned}
$$

and $\tilde{W}_{+\sigma}^{+\sigma}\left(\chi_{\mathrm{N}}, z=0\right)$ can be obtained by simply set $z \rightarrow 0$. 


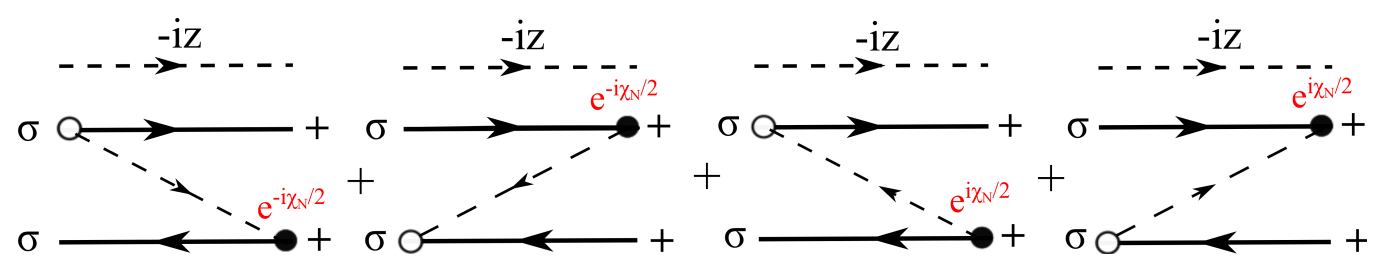

Figure B.3: Diagrams contributing to the kernel element $\tilde{W}_{+\sigma}^{+\sigma}(\omega)$. All vertices taking place in the non-counting interval are represented by open circle. These are getting contracted with vertices in the counting interval (black dots). All counting vertices get a prefactor which includes the counting field $\chi_{N}$. Every vertex on the upper keldysh contour gets a factor $e^{i \chi_{N} / 2}$ and a vertex on the lower contour $e^{-i \chi_{N} / 2}$, respectively. Note, that for every vertex where the tunneling line leaves the factor gets complex conjugated. 


\section{Appendix C}

\section{Finite-frequency noise in the unidirectional transport regime}

In the following we provide analytic expressions for the self-energy kernels and the full propagator in the unidirectional transport contributing to the finite-frequency noise for a quantum dot coupled to one normal and one superconducting lead. The results are presented and discussed in Chapter 4. The diagrammatic approach for noise has been introduced in Sec. 3.4. In the unidirectional transport regime, the applied bias voltage to the normal conducting $\mu_{\mathrm{N}}$ is assumed to be much larger than all other energy scales except the superconducting gap $\Delta$. Hence, all Fermi functions $f\left(E_{ \pm, \pm}-\right.$ $\left.\mu_{\mathrm{N}}\right)\left(E_{ \pm, \pm}\right.$are the Andreev addition energies) can be approximated by 1 . Therefore all transition rates simplify and all kernels in Eq. 3.34 become frequency independent.

The reduced density matrix of the system is is given by

$$
\boldsymbol{P}_{\text {stat }}=\left(\begin{array}{cccc}
P_{+}^{+} & P_{-}^{+} & 0 & 0 \\
P_{+}^{-} & P_{-}^{-} & 0 & 0 \\
0 & 0 & P_{\uparrow}^{\uparrow} & 0 \\
0 & 0 & 0 & P_{\downarrow}^{\downarrow}
\end{array}\right)
$$

where the diagonal elements are the occupation probabilities to find the dot singly occupied $|\sigma\rangle$ or in one of the BCS-like states $| \pm\rangle$. The off-diagonal 
elements describe the coherent superposition of the states with different particle numbers. The elements of the reduced density matrix can be obtained by the master equation, $\boldsymbol{W}(\omega=0) \boldsymbol{P}_{\text {stat }}=0$.

The free propagator of the system, defined in Eq. 3.24 is given by

$$
\Pi_{0}(\omega)=\left(\begin{array}{cccccc}
\frac{i}{-\omega+i 0^{+}} & 0 & 0 & 0 & 0 & 0 \\
0 & \frac{i}{-\omega+i 0^{+}} & 0 & 0 & 0 & 0 \\
0 & 0 & \frac{i}{-\omega+i 0^{+}} & 0 & 0 & 0 \\
0 & 0 & 0 & \frac{i}{-\omega+i 0^{+}} & 0 & 0 \\
0 & 0 & 0 & 0 & \frac{i}{-2 \epsilon_{\mathrm{A}}-\omega+i 0^{+}} & 0 \\
0 & 0 & 0 & 0 & 0 & \frac{i}{2 \epsilon_{\mathrm{A}}-\omega+i 0^{+}}
\end{array}\right)
$$

Note, that for technical reason it is convenient to express the density matrix as a vector $\boldsymbol{P}_{\text {stat }}=\left(P_{+}^{+}, P_{-}^{-}, P_{\uparrow}^{\uparrow}, P_{\downarrow}^{\downarrow}, P_{-}^{+}, P_{+}^{-}\right)^{T}$. We find for the the self-energy kernel $\boldsymbol{W}$ in the high bias regime,

$$
\boldsymbol{W}=\left(\begin{array}{cccccc}
-\Gamma_{\mathrm{N}}\left(1-\frac{\delta}{2 \epsilon_{\mathrm{A}}}\right) & 0 & \frac{\Gamma_{\mathrm{N}}}{2}\left(1+\frac{\delta}{2 \epsilon_{\mathrm{A}}}\right) & \frac{\Gamma_{\mathrm{N}}}{2}\left(1+\frac{\delta}{2 \epsilon_{\mathrm{A}}}\right) & -\frac{\Gamma_{\mathrm{N}} \Gamma_{\mathrm{S}}}{4 \epsilon_{\mathrm{A}}} & -\frac{\Gamma_{\mathrm{N}} \Gamma_{\mathrm{S}}}{4 \epsilon_{\mathrm{A}}} \\
0 & -\Gamma_{\mathrm{N}}\left(1+\frac{\delta}{2 \epsilon_{\mathrm{A}}}\right) & \frac{\Gamma_{\mathrm{N}}}{2}\left(1-\frac{\delta}{2 \epsilon_{\mathrm{A}}}\right) & \frac{\Gamma_{\mathrm{N}}}{2}\left(1-\frac{\delta}{2 \epsilon_{\mathrm{A}}}\right) & -\frac{\Gamma_{\mathrm{N}} \Gamma_{\mathrm{S}}}{4 \epsilon_{\mathrm{A}}} & -\frac{\Gamma_{\mathrm{N}} \Gamma_{\mathrm{S}}}{4 \epsilon_{\mathrm{A}}} \\
\frac{\Gamma_{\mathrm{N}}}{2}\left(1-\frac{\delta}{2 \epsilon_{\mathrm{A}}}\right) & \frac{\Gamma_{\mathrm{N}}}{2}\left(1+\frac{\delta}{2 \epsilon_{\mathrm{A}}}\right) & -\Gamma_{\mathrm{N}} & 0 & \frac{\Gamma_{\mathrm{N}} \Gamma_{\mathrm{S}}}{4 \epsilon_{\mathrm{A}}} & \frac{\Gamma_{\mathrm{N}} \Gamma_{\mathrm{S}}}{4 \epsilon_{\mathrm{A}}} \\
\frac{\Gamma_{\mathrm{N}}}{2}\left(1-\frac{\delta}{2 \epsilon_{\mathrm{A}}}\right) & \frac{\Gamma_{\mathrm{N}}}{2}\left(1+\frac{\delta}{2 \epsilon_{\mathrm{A}}}\right) & 0 & -\Gamma_{\mathrm{N}} & \frac{\Gamma_{\mathrm{N}} \Gamma_{\mathrm{S}}}{4 \epsilon_{\mathrm{A}}} & \frac{\Gamma_{\mathrm{N}} \Gamma_{\mathrm{S}}}{4 \epsilon_{\mathrm{A}}} \\
-\frac{\Gamma_{\mathrm{N}} \Gamma_{\mathrm{S}}}{4 \epsilon_{\mathrm{A}}} & -\frac{\Gamma_{\mathrm{N}} \Gamma_{\mathrm{S}}}{4 \epsilon_{\mathrm{A}}} & -\frac{\Gamma_{\mathrm{N}} \Gamma_{\mathrm{S}}}{4 \epsilon_{\mathrm{A}}} & -\frac{\Gamma_{\mathrm{N}} \Gamma_{\mathrm{S}}}{4 \epsilon_{\mathrm{A}}} & -\Gamma_{\mathrm{N}} & 0 \\
-\frac{\Gamma_{\mathrm{N}} \Gamma_{\mathrm{S}}}{4 \epsilon_{\mathrm{A}}} & -\frac{\Gamma_{\mathrm{N}} \Gamma_{\mathrm{S}}}{4 \epsilon_{\mathrm{A}}} & -\frac{\Gamma_{\mathrm{N}} \Gamma_{\mathrm{S}}}{4 \epsilon_{\mathrm{A}}} & -\frac{\Gamma_{\mathrm{N}} \Gamma_{\mathrm{S}}}{4 \epsilon_{\mathrm{A}}} & 0 & -\Gamma_{\mathrm{N}}
\end{array}\right)
$$

Using the master equation, $\boldsymbol{W}(\omega=0) \boldsymbol{P}_{\text {stat }}=0$, we calculate the following elements of the reduced density matrix,

$$
\begin{aligned}
P_{+}^{+} & =\frac{1}{16}\left(\frac{\left(\delta+2 \epsilon_{\mathrm{A}}\right)^{2}}{\epsilon_{\mathrm{A}}^{2}}+\frac{\Gamma_{\mathrm{N}}^{2} \Gamma_{\mathrm{S}}^{2}}{\Gamma_{\mathrm{N}}^{2} \epsilon_{\mathrm{A}}^{2}+4 \epsilon_{\mathrm{A}}^{2}}\right) \\
P_{-}^{-} & =\frac{1}{16}\left(\frac{\left(\delta-2 \epsilon_{\mathrm{A}}\right)^{2}}{\epsilon_{\mathrm{A}}^{2}}+\frac{\Gamma_{\mathrm{N}}^{2} \Gamma_{\mathrm{S}}^{2}}{\Gamma_{\mathrm{N}}^{2} \epsilon_{\mathrm{A}}^{2}+4 \epsilon_{\mathrm{A}}^{2}}\right) \\
P_{\sigma}^{\sigma} & =\frac{1}{16}\left(4-\frac{\delta^{2}+\Gamma_{\mathrm{S}}^{2}}{\epsilon_{\mathrm{A}}^{2}}+\frac{4 \Gamma_{\mathrm{S}}^{2}}{\Gamma_{\mathrm{N}}^{2}+4 \epsilon_{\mathrm{A}}^{2}}\right)
\end{aligned}
$$




$$
\begin{aligned}
& P_{-}^{+}=-\frac{\Gamma_{\mathrm{N}} \Gamma_{\mathrm{S}}}{4 \Gamma_{\mathrm{N}} \epsilon_{\mathrm{A}}+i 8 \epsilon_{\mathrm{A}}^{2}} \\
& P_{+}^{-}=-\frac{\Gamma_{\mathrm{N}} \Gamma_{\mathrm{S}}}{4 \Gamma_{\mathrm{N}} \epsilon_{\mathrm{A}}-i 8 \epsilon_{\mathrm{A}}^{2}}
\end{aligned}
$$

In order to derive the finite-frequency noise we have to consider diagrams where one or two tunneling vertices are replaced by a current vertex. The kernel $\boldsymbol{W}_{\boldsymbol{I}}$ sums up all diagrams, where note tunnel vertex is replaced by a current vertex and is given by

$$
\boldsymbol{W}_{\boldsymbol{I}}=\left(\begin{array}{cccccc}
0 & 0 & \Gamma_{\mathrm{N}}\left(1+\frac{\delta}{2 \epsilon_{\mathrm{A}}}\right) & \Gamma_{\mathrm{N}}\left(1+\frac{\delta}{2 \epsilon_{\mathrm{A}}}\right) & 0 & 0 \\
0 & 0 & \Gamma_{\mathrm{N}}\left(1-\frac{\delta}{2 \epsilon_{\mathrm{A}}}\right) & \Gamma_{\mathrm{N}}\left(1-\frac{\delta}{2 \epsilon_{\mathrm{A}}}\right) & 0 & 0 \\
\Gamma_{\mathrm{N}}\left(1-\frac{\delta}{2 \epsilon_{\mathrm{A}}}\right) & \Gamma_{\mathrm{N}}\left(1+\frac{\delta}{2 \epsilon_{\mathrm{A}}}\right) & 0 & 0 & \frac{\Gamma_{\mathrm{N}} \Gamma_{\mathrm{S}}}{2 \epsilon_{\mathrm{A}}} & \frac{\Gamma_{\mathrm{N}} \Gamma_{\mathrm{S}}}{2 \epsilon_{\mathrm{A}}} \\
\Gamma_{\mathrm{N}}\left(1-\frac{\delta}{2 \epsilon_{\mathrm{A}}}\right) & \Gamma_{\mathrm{N}}\left(1+\frac{\delta}{2 \epsilon_{\mathrm{A}}}\right) & 0 & 0 & \frac{\Gamma_{\mathrm{N}} \Gamma_{\mathrm{S}}}{2 \epsilon_{A}} & \frac{\Gamma_{\mathrm{N}} \Gamma_{S}}{2 \epsilon_{\mathrm{A}}} \\
0 & 0 & -\frac{\Gamma_{\mathrm{N}} \Gamma_{\mathrm{S}}}{2 \epsilon_{\mathrm{A}}} & -\frac{\Gamma_{\mathrm{N}} \Gamma_{\mathrm{S}}}{2 \epsilon_{\mathrm{A}}} & 0 & 0 \\
0 & 0 & -\frac{\Gamma_{\mathrm{N}} \Gamma_{\mathrm{S}}}{2 \epsilon_{\mathrm{A}}} & -\frac{\Gamma_{\mathrm{N}} \Gamma_{\mathrm{S}}}{2 \epsilon_{\mathrm{A}}} & 0 & 0
\end{array}\right)
$$

The kernel $\boldsymbol{W}_{\boldsymbol{I}}$ contains all diagrams with both current vertices in the same irreducible block and we derive

$$
\boldsymbol{W}_{\boldsymbol{I} \boldsymbol{I}}=\left(\begin{array}{cccccc}
\Gamma_{\mathrm{N}}\left(1-\frac{\delta}{2 \epsilon_{\mathrm{A}}}\right) & 0 & \frac{\Gamma_{\mathrm{N}}}{2}\left(1+\frac{\delta}{2 \epsilon_{\mathrm{A}}}\right) & \frac{\Gamma_{\mathrm{N}}}{2}\left(1+\frac{\delta}{2 \epsilon_{\mathrm{A}}}\right) & \frac{\Gamma_{\mathrm{N}} \Gamma_{\mathrm{S}}}{4 \epsilon_{\mathrm{A}}} & \frac{\Gamma_{\mathrm{N}} \Gamma_{\mathrm{S}}}{4 \epsilon_{\mathrm{A}}} \\
0 & \Gamma_{\mathrm{N}}\left(1+\frac{\delta}{2 \epsilon_{\mathrm{A}}}\right) & \frac{\Gamma_{\mathrm{N}}}{2}\left(1-\frac{\delta}{2 \epsilon_{\mathrm{A}}}\right) & \frac{\Gamma_{\mathrm{N}}}{2}\left(1-\frac{\delta}{2 \epsilon_{\mathrm{A}}}\right) & \frac{\Gamma_{\mathrm{N}} \Gamma_{\mathrm{S}}}{4 \epsilon_{\mathrm{A}}} & \frac{\Gamma_{\mathrm{N}} \Gamma_{\mathrm{S}}}{4 \epsilon_{\mathrm{A}}} \\
\frac{\Gamma_{\mathrm{N}}}{2}\left(1-\frac{\delta}{2 \epsilon_{\mathrm{A}}}\right) & \frac{\Gamma_{\mathrm{N}}}{2}\left(1+\frac{\delta}{2 \epsilon_{\mathrm{A}}}\right) & \Gamma_{\mathrm{N}} & 0 & \frac{\Gamma_{\mathrm{N}} \Gamma_{\mathrm{S}}}{4 \epsilon_{\mathrm{A}}} & \frac{\Gamma_{\mathrm{N}} \Gamma_{\mathrm{S}}}{4 \epsilon_{\mathrm{A}}} \\
\frac{\Gamma_{\mathrm{N}}}{2}\left(1-\frac{\delta}{2 \epsilon_{\mathrm{A}}}\right) & \frac{\Gamma_{\mathrm{N}}}{2}\left(1+\frac{\delta}{2 \epsilon_{\mathrm{A}}}\right) & 0 & \Gamma_{\mathrm{N}} & \frac{\Gamma_{\mathrm{N}} \Gamma_{\mathrm{S}}}{4 \epsilon_{\mathrm{A}}} & \frac{\Gamma_{\mathrm{N}} \Gamma_{\mathrm{S}}}{4 \epsilon_{\mathrm{A}}} \\
\frac{\Gamma_{\mathrm{N}} \Gamma_{\mathrm{S}}}{4 \epsilon_{\mathrm{A}}} & \frac{\Gamma_{\mathrm{N}} \Gamma_{\mathrm{S}}}{4 \epsilon_{\mathrm{A}}} & -\frac{\Gamma_{\mathrm{N}} \Gamma_{\mathrm{S}}}{4 \epsilon_{\mathrm{A}}} & -\frac{\Gamma_{\mathrm{N}} \Gamma_{\mathrm{S}}}{4 \epsilon_{\mathrm{A}}} & \Gamma_{\mathrm{N}} & 0 \\
\frac{\Gamma_{\mathrm{N}} \Gamma_{\mathrm{S}}}{4 \epsilon_{\mathrm{A}}} & \frac{\Gamma_{\mathrm{N}} \Gamma_{\mathrm{S}}}{4 \epsilon_{\mathrm{A}}} & -\frac{\Gamma_{\mathrm{N}} \Gamma_{\mathrm{S}}}{4 \epsilon_{\mathrm{A}}} & -\frac{\Gamma_{\mathrm{N}} \Gamma_{\mathrm{S}}}{4 \epsilon_{\mathrm{A}}} & 0 & \Gamma_{\mathrm{N}}
\end{array}\right)
$$

Now we are able to calculate the current and the current noise by using Eq. (3.26) and (3.34). The current in the high bias limit yields

$$
I=\Gamma_{\mathrm{N}}-\frac{\Gamma_{\mathrm{N}}\left(\Gamma_{\mathrm{S}}^{2}+\delta^{2}\right)}{4 \epsilon_{\mathrm{A}}^{2}}+\frac{\Gamma_{\mathrm{N}} \Gamma_{\mathrm{S}}^{2}}{\Gamma_{\mathrm{N}}^{2}+4 \epsilon_{\mathrm{A}}^{2}}
$$


Since we consider a strong coupling to the superconductor $\Gamma_{\mathrm{S}} \gg \Gamma_{\mathrm{N}}$, the expression simplifies to

$$
I_{\text {uni }}=\Gamma_{\mathrm{N}} \frac{\Gamma_{\mathrm{S}}^{2}}{4 \epsilon_{\mathrm{A}}^{2}} .
$$

and we find for the Fano factor in the unidirectional transport regime for $\Gamma_{\mathrm{S}} \gg \Gamma_{\mathrm{N}}$,

$$
\begin{aligned}
& \frac{S(\omega)}{2 I_{\mathrm{uni}}}=1+\frac{\Gamma_{\mathrm{N}}^{2} \delta^{2}}{4 \epsilon_{\mathrm{A}}^{2}\left(\Gamma_{\mathrm{N}}^{2}+\omega^{2}\right)}- \\
& \quad \frac{1}{2} \frac{\Gamma_{\mathrm{S}}^{2}}{4 \epsilon_{\mathrm{A}}^{2}}\left[\frac{\Gamma_{\mathrm{N}}^{2}}{\Gamma_{\mathrm{N}}^{2}+\left(\omega-2 \epsilon_{\mathrm{A}}\right)^{2}}\left(1-\frac{\omega-2 \epsilon_{\mathrm{A}}}{\epsilon_{\mathrm{A}}}\right)+\frac{\Gamma_{\mathrm{N}}^{2}}{\Gamma_{\mathrm{N}}^{2}+\left(2 \epsilon_{\mathrm{A}}+\omega\right)^{2}}\left(1+\frac{\omega+2 \epsilon_{\mathrm{A}}}{\epsilon_{\mathrm{A}}}\right)\right] .
\end{aligned}
$$




\section{List of Publications}

(A) Josephson current through interacting double quantum dots with spin-orbit coupling,

S. Droste, S. Andergassen and J. Splettstoesser,

J. Phys.: Condens. Matter 24, 415301 (2012).

(B) Finite-frequency noise in a quantum dot with normal and superconducting leads,

S. Droste, J. Splettstoesser and Michele Governale, Phys. Rev. B 91, 125401 (2015).

(C) Finite-time full counting statistics and factorial cumulants for transport through a quantum dot with normal and superconducting leads, S. Droste and Michele Governale, in preparation (2015).

Chapter 3 and 4 contain parts of reference (B) and Chapter 3 and 5 of reference (C). 


\section{Bibliography}

[1] R. C. Ashoori, Nature 379, 413 (1996).

[2] S. D. Franceschi, L. Kouwenhoven, C. Schönenberger, and W. Wernsdorfer, Nature Nanotechnology 5, 703 (2010).

[3] A. Martin-Rodero and A. Levy Yeyati, Adv. Phys. 60, 899 (2011).

[4] M. Leijnse and K. Flensberg, Phys. Rev. B 86, 134528 (2012).

[5] B. Sothmann, J. Li, and M. Büttiker, New. J. Phys. 15, 085018 (2013).

[6] A. R. Wright and M. Veldhorst, Phys. Rev. Lett. 111, 096801 (2013).

[7] A. Brunetti, A. Zazunov, A. Kundu, and R. Egger, Phys. Rev. B 88, 144515 (2013).

[8] B. Sothmann, S. Weiss, M. Governale, and J. König, Phys. Rev. B 90, 220501(R) (2014).

[9] L. Hofstetter, S. Csonka, J. Nygård, and C. Schönenberger, Nature 461, 960 (2009).

[10] L. G. Herrmann, F. Portier, P. Roche, A. L. Yeyati, T. Kontos, and C. Strunk, Phys. Rev. Lett. 104, 026801 (2010).

[11] A. Andreev, Sov. Phys. JETP 19, 1228 (1964). 
[12] T. Sand-Jespersen, J. Paaske, B. M. Andersen, K. Grove-Rasmussen, H. I. Jørgensen, M. Aagesen, C. B. Sørensen, P. E. Lindelof, K. Flensberg, and J. Nygård, Phys. Rev. Lett. 99, 126603 (2007).

[13] A. Eichler, M. Weiss, S. Oberholzer, C. Schönenberger, A. Levy Yeyati, J. C. Cuevas, and A. Martin-Rodero, Phys. Rev. Lett. 99, 126602 (2007).

[14] R. S. Deacon, Y. Tanaka, A. Oiwa, R. Sakano, K. Yoshida, K. Shibata, K. Hirakawa, and S. Tarucha, Phys. Rev. Lett. 104, 076805 (2010).

[15] J.-D. Pillet, C. H. L. Quay, P. Morfin, C. Bena, A. L. Yeyati, and P. Joyez, Nature Physics 6, 965 (2010).

[16] T. Dirks, T. L. Hughes, S. Lal, B. Uchoa, Y.-F. Chen, C. Chialvo, P. M. Goldbart, and N. Mason, Nature Physics 7, 386 (2011).

[17] E. Lee, X. Jiang, R. Aguado, G. Katsaros, C. Lieber, and S. De Franceschi, Phys. Rev. Lett. 109, 186802 (2012).

[18] J.-D. Pillet, P. Joyez, R. Žitko, and M. Goffman, Phys. Rev. B 88, 045101 (2013).

[19] W. Chang, V. E. Manucharyan, T. Jespersen, J. Nygård, and C. Marcus, Phys. Rev. Lett. 110, 217005 (2013).

[20] E. J. H. Lee, X. Jiang, M. Houzet, R. Aguado, C. M. Lieber, and S. De Franceschi, Nature Nanotechnology 9, 79 (2014).

[21] A. Kumar, M. Gaim, D. Steininger, A. L. Yeyati, A. Martin-Rodero, A. K. Hüttel, and C. Strunk, Phys. Rev. B 89, 075428 (2014).

[22] J. Schindele, A. Baumgartner, R. Maurand, M. Weiss, and C. Schönenberger, Phys. Rev. B 89, 045422 (2014).

[23] H. Nyquist, Phys. Rev. 32, 110 (1928). 
[24] A. A. Clerk, M. H. Devoret, S. M. Girvin, F. Marquardt, and R. J. Schoelkopf, Rev. Mod. Phys. 82, 1155 (2010).

[25] B. Reulet, J. Senzier, and D. E. Prober, Phys. Rev. Lett. 91, 196601 (2003).

[26] L. S. Levitov and G. B. Lesovik, JETP Lett. 58, 230 (1993).

[27] L. S. Levitov, H.-W. Lee, and G. B. Lesovik, J. Math. Phys. 37, 4845 (1996).

[28] S. Gustavsson, R. Leturcq, B. Simovic, R. Schleser, T.Ihn, P. Studerus, K. Ensslin, D. C. Driscoll, and A. C. Gossard, Phys. Rev. Lett. 96, 076605 (2006).

[29] D. V. Averin, J. Appl. Phys. 73, 2593 (1993).

[30] G.-H. Ding and T.-K. Ng, Phys. Rev. B 56, 15521(R) (1997).

[31] R. Aguado and L. P. Kouwenhoven, Phys. Rev. Lett. 84, 1986 (2000).

[32] Y. Blanter and M. Büttiker, Phys. Rep. 336, 1 (2000).

[33] H. A. Engel and D. Loss, Phys. Rev. Lett. 93, 136602 (2004).

[34] M. Braun, J. König, and J. Martinek, Phys. Rev. B 74, 075328 (2006).

[35] O. Entin-Wohlman, Y. Imry, S. A. Gurvitz, and A. Aharony, Phys. Rev. B 75, 193308 (2007).

[36] E. A. Rothstein, O. Entin-Wohlman, and A. Aharony, Phys. Rev. B 79, 075307 (2009).

[37] N. Gabdank, E. A. Rothstein, O. Entin-Wohlman, and A. Aharony, Phys. Rev. B 84, 235435 (2011).

[38] C. P. Orth, D. F. Urban, and A. Komnik, Phys. Rev. B 86, 125324 (2012). 
[39] A. Branschädel, E. Boulat, H. Saleur, and P. Schmitteckert, Phys. Rev. Lett. 105, 146805 (2010).

[40] D. Marcos, C. Emary, T. Brandes, and R. Aguado, Phys. Rev. B 83, $125426(2011)$.

[41] K. Joho, S. Maier, and A. Komnik, Phys. Rev. B 86, 155304 (2012).

[42] B. Sothmann, J. König, and A. Kadigrobov, Phys. Rev. B 82, 205314 (2010).

[43] J. Jin, W.-M. Zhang, X.-Q. Li, and Y. Yan, arXiv:condmat/1105.0136v2 (2012).

[44] S. Y. Müller, M. Pletyukhov, D. Schuricht, and S. Andergassen, Phys. Rev. B 87, 245115 (2013).

[45] G.-H. Ding and B. Dong, Phys. Rev. B 87, 235303 (2013).

[46] P. G. Kirton, A. D. Armour, M. Houzet, and F. Pistolesi, Phys. Rev. B 86, 081305 (2012).

[47] H. Soller and A. Komnik, EPL 106, 37009 (2014).

[48] C. P. Moca, P. Simon, C.-H. Chung, and G. Zarand, Phys. Rev. B 89, $155138(2014)$

[49] M. Governale, M. G. Pala, and J. König, Phys. Rev. B 77, 134513 (2008).

[50] R. H. Koch, D. J. Van Harlingen, and J. Clarke, Phys. Rev. B 26, 74 (1982).

[51] R. J. Schoelkopf, P. J. Burke, A. A. Kozhevnikov, D. E. Prober, and M. J. Rooks, Phys. Rev. Lett. 78, 3370 (1997).

[52] R. Deblock, E. Onac, L. Gurevich, and L. P. Kouwenhoven, Science 301, 203 (2003). 
[53] E. Onac, F. Balestro, L. H. W. van Beveren, U. Hartmann, Y. V. Nazarov, and L. P. Kouwenhoven, Phys. Rev. Lett. 96, 176601 (2006).

[54] P.-M. Billangeon, F. Pierre, H. Bouchiat, and R. Deblock, Phys. Rev. Lett. 96, 136804 (2006).

[55] P.-M. Billangeon, F. Pierre, H. Bouchiat, and R. Deblock, Phys. Rev. Lett. 98, 126802 (2007).

[56] E. Zakka-Bajjani, J. Ségala, F. Portier, P. Roche, D. C. Glattli, A. Cavanna, and Y. Jin, Phys. Rev. Lett. 99, 236803 (2007).

[57] J. Gabelli and B. Reulet, Phys. Rev. Lett. 100, 026601 (2008).

[58] N. Ubbelohde, C. Fricke, C. Flindt, F. Hohls, and R. J. Haug, Nat. Commun. 3, 612 (2012).

[59] V. F. Maisi, D. Kambly, C. Flindt, and J. P. Pekola, Phys. Rev. Lett. 12, 036801 (2014).

[60] C. Flindt, T. Novotny, A. Braggio, M. Sassetti, and A.-P. Jauho, Phys. Rev. Lett. 100, 150601 (2008).

[61] C. Flindt, C. Fricke, F. Hohls, T. Novotny, K. Netocny, T. Brandes, and R. J. Haug, Proc. Natl. Acad. Sci USA 106, 10116 (2009).

[62] D. Marcos, C. Emary, T. Brandes, and R. Aguado, New Journal of Physics 12, 123009 (2010).

[63] C. Emary and R. Aguado, Phys. Rev. B 84, 085425 (2011).

[64] J. König, H. Schoeller, and G. Schön, Phys. Rev. Lett. 76, 1715 (1996).

[65] J. König, J. Schmid, H. Schoeller, and G. Schön, Phys. Rev. B 54, 16820 (1996).

[66] J. König, Quantum Fluctuations in the Single-Electron Transistor (Shaker, Aachen, 1999). 
[67] L. P. Kouwenhoven, D. G. Austing, and S. Tarucha, Rep. Prog. Phys. 64, $701(2001)$.

[68] D. Goldhaber-Gordon, H. Shtrikman, D. Mahalu, D. Abusch-Magder, U. Meirav, and M. A. Kastner, Nature 391, 156 (1998).

[69] L. Vandersypen, R. Hanson, L. W. van Beveren, J. Elzerman, J. Greidanus, S. D. Franceschi, and L. Kouwenhoven, Quantum Computing and Quantum Bits in Mesoscopic Systems, Kluwer Academic Plenum Publishers (2003).

[70] S. Tarucha, D. G. Austing, T. Honda, R. J. van der Hage, and L. P. Kouwenhoven, Phys. Rev. Lett. 77, 3613 (1996).

[71] C. Stampfer, E. Schurtenberger, F. Molitor, J. Güttinger, T. Ihn, and K. Ensslin, Nano Letters 8, 2378 (2008).

[72] H. G. Zhang, H. Hu, Y. Pan, J. H. Mao, M. Gao, H. M. Guo, S. X. Du, T. Greber, and H.-J. Gao, J. Phys.: Condens. Matter 22, 302001 (2010).

[73] C. Volk, C. Neumann, S. Kazarski, S. Fringes, S. Engels, F. Haupt, A. Müller, and C. Stampfer, Nat. Commun. 4, 1753 (2013).

[74] J. Nygård and D. H. Cobden, Appl. Phys. Lett. 79, 4216 (2001).

[75] P. Jarillo-Herrero, S. Sapmaz, C. Dekker, L. P. Kouwenhoven, and H. S. J. van der Zant, Nature 429, 389 (2004).

[76] S. Sapmaz, P. Jarillo-Herrero, . P. Kouwenhoven, and H. S. J. van der Zant, Semicond. Sci. Technol. 21, S52 (2006).

[77] M. T. González, S. Wu, R. Huber, S. J. van der Molen, C. Schönenberger, and M. Calame, Nano Letters 6, 2238 (2006).

[78] M. Hofheinz, X. Jehl, M. Sanquer, G. Molas, M. Vinet, and S. Deleonibus, Appl. Phys. Lett. 89, 143504 (2006). 
[79] M. Fuechsle, J. A. Miwa, S. Mahapatra, H. Ryu, S. Lee, O. Warschkow, L. C. L. Hollenberg, G. Klimeck, and M. Y. Simmons, Nature Nanotechnology 7, 242 (2012).

[80] V. S. Pribiag, S. Nadj-Perge, S. M. Frolov, J. W. G. van den Berg, I. van Weperen, S. R. Plissard, E. P. A. M. Bakkers, and L. .P. Kouwenhoven, Nature Nanotechnology 8, 170 (2013).

[81] M. T. Björk, C. Thelander, A. E. Hansen, L. E. Jensen, M. W. L. and L. R. Wallenberg, and L. Samuelson, Nano Letters 4, 1621 (2004).

[82] L. H. W. van Beveren, R. Hanson, I. T. Vink, F. H. L. Koppens, L. P. Kouwenhoven, and L. M. K. Vandersypen, New. J. Phys. 7, 182 (2005).

[83] D. Bimberg, Electronics Letters 44, 168 (2008).

[84] D. Loss and D. P. DiVincenzo, Phys. Rev. A 57, 120 (1998).

[85] M. Kroutvar, Y. Ducommun, D. Heiss, M. Bichler, D. Schuh, G. Abstreiter, and J. J. Finley, Nature 432, 81 (2004).

[86] T. D. Ladd, F. Jelezko, R. Laflamme, Y. Nakamura, C. Monroe, and J. L. O'Brien, Nature 464, 45 (2010).

[87] D. Bimberg, E. Stock, A. Lochmann, A. Schliwa, W. Unrau, M. Münnix, S. Rodt, A. I. Toropov, A. Bakarov, A. K. Kalagin, and V. A. Haisler, IEEE Photonics J. 1, 58 (2009).

[88] P. W. Anderson, Phys. Rev. 124, 41 (1961).

[89] L. P. Kouwenhoven, T. H. Oosterkamp, M. W. S. Danoesastro, M. Eto, D. G. Austing, T. Honda, and S. Tarucha, Science 278, 1788 (1997).

[90] D. V. Averin and Y. V. Nazarov, Phys. Rev. Lett. 65, 2446 (1990).

[91] J. Kondo, Prog. Theor. Phys. 32, 37 (1964). 
[92] T. K. Ng and P. A. Lee, Phys. Rev. Lett. 61, 1768 (1988).

[93] L. I. Glazman and M. E. Raikh, JETP Lett. 47, 452 (1988).

[94] D. Futterer, J. Swiebodzinski, M. G. Governale, and J. König, Phys. Rev. B 87, 014509 (2013).

[95] W. Schottky, Annalen der Physik 57, 541 (1918).

[96] U. Fano, Phys. Rev. 72, 26 (1947).

[97] F. Lefloch, C. Hoffmann, M. Sanquer, and D. Quirion, Phys. Rev. Lett. 90, 067002 (2003).

[98] W. W. Xue, Z. Ji, F. Pan, J. Stettenheim, M. P. Blencowe, and A. J. Rimberg, Nat. Phys. 5, 660 (2009).

[99] J. Basset, H. Bouchiat, and R. Deblock, Phys. Rev. Lett. 105, 166801 (2010).

[100] Y. V. Nazarov, Ann. Phys. 8, 507 (1999).

[101] M. Kindermann and Y. V. Nazarov, Phys. Rev. Lett. 91, 136802 (2003).

[102] D. A. Bragrets and Y. V. Nazarov, Phys. Rev. B 67, 085316 (2003).

[103] A. Braggio, J. König, and R. Fazio, Phys. Rev. Lett. 96, 026805 (2006).

[104] C. Emary, D. Marcos, R. Aguado, and T. Brandes, Phys. Rev. B 76, 161404(R) (2007).

[105] Y. Bomze, G. Gershon, D. Shovkun, L. S. Levitov, and M. Reznikov, Phys. Rev. Lett. 95, 176601 (2005).

[106] J. Bylander, T. Duty, and P. Delsing, Nature 434, 361 (2005).

[107] T. Fujisawa, T. Hayashi, R. Tomita, and Y. Hirayama, Science 312, 1634 (2006). 
[108] C. Fricke, F. Hohls, W. Wegscheider, and R. J. Haug, Phys Rev. B 76, 155307 (2007).

[109] A. V. Timofeev, M. Meschke, J. T. Peltonen, T. T. Heikkilä, and J. P. Pekola, Phys. Rev. Lett. 98, 207001 (2007).

[110] G. Gershon, Y. Bomze, E. V. Sukhorukov, and M. Reznikov, Phys. Rev. Lett. 101, 016803 (2008).

[111] S. Gustavsson, R. Leturcq, M. Studer, I. Shorubalko, T. Ihn, K. Ensslin, D. C. Driscoll, and A. C. Gossard, Surface Science Reports 64, $191(2009)$.

[112] J. Gabelli and B. Reulet, Phys. Rev. B 80, 161203(R) (2009).

[113] C. Fricke, F. Hohls, C. Emary, and R. J. Haug, Physica E 42, 848 (2010).

[114] C. Fricke, F. Hohls, N. Sethubalasubramanian, L. Fricke, and R. J. Haug, Appl, Phys. Lett. 96, 202103 (2010).

[115] H. K. Onnes, Leiden Comm 120b, 122b, 124c (1911).

[116] W. Meissner and R. Ochsenfeld, Naturwissenschaften 21, 787 (1933).

[117] J. Bardeen, L. N. Cooper, and J. R. Schrieffer, Phys. Rev. 108, 1175 (1957).

[118] G. Bednorz and K. A. Müller, Z. Phys. B 64, 189 (1986).

[119] C. W. J. Beenakker, Rev. Mod. Phys. 69, 731 (1997).

[120] M. Tinkham, Introduction to Superconductivity, Dover Publications (2004).

[121] L. N. Cooper, Phys. Rev. 104, 1189 (1956).

[122] W. L. McMillan, Phys. Rev. 175, 537 (1968). 
[123] I. Kulik, Sov. Phys. JETP (USSR) 30, 944 (1970).

[124] B. D. Josephson, Phys. Lett. 1, 251 (1962).

[125] V. V. Ryazanov, V A. Oboznov, A. Y. Rusanov, A. V. Veretennikov, A. A. Golubov, and J. Aarts, Phys. Rev. Lett. 86, 2427 (2001).

[126] A. A. Bannykh, J. Pfeiffer, V. S. Stolyarov, I. E. Batov, V. V. Ryazanov, and M. Weides, Phys. Rev. B 79, 054501 (2009).

[127] J. W. A. Robinson, S. Piano, G. Burnell, C. Bell, and M. G. Blamire, Phys. Rev. Lett. 97, 177003 (2006).

[128] M. Weides, M. Kemmler, E. Goldobin, D. Koelle, R. Kleiner, H. Kohlstedt, and A. Buzdin, Appl. Phys. Lett. 97, 177003 (2006).

[129] L. Hofstetter, S. Csonka, A. Baumgartner, G. Fülöp, S. d'Hollosy, J. Nygård, and C. Schönenberger, Phys. Rev. Lett. 107, 136801 (2011).

[130] M. Buitelaar, T. Nussbaumer, and C. Schönenberger, Phys. Rev. Lett. 89, 256801 (2002).

[131] M. Buitelaar, W. Belzig, T. Nussbaumer, B. Babić, C. Bruder, and C. Schönenberger, Phys. Rev. Lett. 91, 057005 (2003).

[132] J.-P. Cleuziou, W. Wernsdorfer, V. Bouchiat, T. Ondarcuhu and M. Monthioux, Nature Nanotechnology 1, 53 (2006).

[133] P. Jarillo-Herrero, J. A. van Dam, and L. P. Kouwenhoven, Nature 439, 953 (2006).

[134] H. Jørgensen, K. Grove-Rasmussen, T. Novotný, K. Flensberg, and P. Lindelof, Phys. Rev. Lett. 96, 207003 (2006).

[135] K. Grove-Rasmussen, H. I. Jørgensen, and P. E. Lindelof, New. J. Phys. 9, 124 (2007). 
[136] H. I. Jørgensen, T. Novotny, K. Grove-Rasmussen, K. Flensberg, and P. E. Lindelof, Nano Letters 7, 2441 (2007).

[137] A. Eichler, R. Deblock, M. Weiss, C. Karrasch, V. Meden, C. Schönenberger, and H. Bouchiat, Phys. Rev. B 79, 161407 (2009).

[138] R. Deacon, Y. Tanaka, A. Oiwa, R. Sakano, K. Yoshida, K. Shibata, K. Hirakawa, and S. Tarucha, Phys. Rev. B 81, 121308 (2010).

[139] J. A. van Dam, Y. V. Nazarov, E. P. A. M. Bakkers, S. De Franceschi, and L. P. Kouwenhoven, Nature 442, 667 (2006).

[140] D. C. Ralph, C. T. Black, and M. Tinkham, Phys. Rev. Lett. 74, 3241 (1995).

[141] J. Clarke and F. K. Wilhelm, Nature 453, 1031 (2008).

[142] A. Zazunov, V. S. Shumeiko, E. N. Bratus', J. Lantz, and G. Wendin, Phys. Rev. Lett. 90, 087003 (2003).

[143] C. W. J. Beenakker, Annu. Rev. Con. Mat. Phys. 4, 113 (2013).

[144] A. Thielmann, M. H. Hettler, J. König, and G. Schön, Phys. Rev. B 68, 115105 (2003).

[145] A. V. Rozhkov and D. P. Arovas, Phys. Rev. B 62, 6687 (2000).

[146] L. V. Keldysh, JETP, 20, 1018 (1965).

[147] U. Gavish, Y. Levinson, and Y. Imry, Phys. Rev. B 62, R10637 (2000).

[148] Y. V. Nazarov and Y. Blanter, 'Quantum Transport, Cambridge University Press (2009).

[149] R. Zwanzig, 'Nonequilibrium statistical mechanics, Oxford University Press (2001). 
[150] G. Schön, Y. Makhlin, and A. Shnirman, Rev. Mod. Phys. 73, 357 (2001).

[151] S. Droste, J. Splettstoesser, and M. Governale, Phys. Rev. B 91, 125401 (2015).

[152] A. S. Wightman, Il Nuovo Cimento B (1971-1996) 110, 751 (1995).

[153] M. G. Pala, M. Governale, and J. König, New. J. Phys. 9, 278 (2007).

[154] A. Braggio, M. Governale, M. G. Pala, and J. König, Solid State Comm. 151, 155 (2011).

[155] L. Rajabi, C. Pöltl, and M. Governale, Phys. Rev. Lett 111, 067002 (2013).

[156] U. Fano, Phys. Rev. 124, 1866 (1961).

[157] D. A. Bragrets and Y. V. Nazarov, Phys. Rev. B 67, 085316 (2003).

[158] C. Flindt, T. Novotný, A. Braggio, and A.-P. Jauho, Phys. Rev. B 82, 155407 (2010).

[159] M. V. Berry, Proc. R. Soc. A 461, 1735 (2005).

[160] K. E. Nagaev, S. Pilgram, and M. Büttiker, Phys. Rev. Lett. 92, 176804 (2004).

[161] S. Pilgram, K. E. Nagaev, and M. Büttiker, Phys. Rev. B 70, 045304 (2004).

[162] M. Abramowitz and I. A. Stegun, Handbook of Mathematical Functions: With Formulas, Graphs, and Mathematical Tables, Dover Publications, Inc., New York, 10 ed. (1972). 